\title{
Rectal cancer: steps towards tailored treatment
}

\author{
Citation for published version (APA):
}

Buijsen, J. (2015). Rectal cancer: steps towards tailored treatment. [Doctoral Thesis, Maastricht University]. Uitgeverij BOXPress. https://doi.org/10.26481/dis.20150701jb

Document status and date:

Published: 01/01/2015

DOI:

10.26481/dis.20150701jb

Document Version:

Publisher's PDF, also known as Version of record

\section{Please check the document version of this publication:}

- A submitted manuscript is the version of the article upon submission and before peer-review. There can be important differences between the submitted version and the official published version of record.

People interested in the research are advised to contact the author for the final version of the publication, or visit the DOI to the publisher's website.

- The final author version and the galley proof are versions of the publication after peer review.

- The final published version features the final layout of the paper including the volume, issue and page numbers.

Link to publication

\footnotetext{
General rights rights.

- You may freely distribute the URL identifying the publication in the public portal. please follow below link for the End User Agreement:

www.umlib.nl/taverne-license

Take down policy

If you believe that this document breaches copyright please contact us at:

repository@maastrichtuniversity.nl

providing details and we will investigate your claim.
}

Copyright and moral rights for the publications made accessible in the public portal are retained by the authors and/or other copyright owners and it is a condition of accessing publications that users recognise and abide by the legal requirements associated with these

- Users may download and print one copy of any publication from the public portal for the purpose of private study or research.

- You may not further distribute the material or use it for any profit-making activity or commercial gain

If the publication is distributed under the terms of Article $25 \mathrm{fa}$ of the Dutch Copyright Act, indicated by the "Taverne" license above, 
Rectal Cancer:

Steps towards tailored treatment

Jeroen Buijsen 
(c) J. Buijsen, Schimmert 2015

ISBN 978-94-6295-224-9

Cover design: Ankie Pellis

Layout: Marlou Mevis

Printed by: $\quad$ Proefschriftmaken.nl | Uitgeverij BOXPress

Published by: Uitgeverij BOXPress, 's-Hertogenbosch

Printing of this thesis was financially supported by ABN AMRO 


\section{Rectal Cancer:}

\section{Steps towards tailored treatment}

PROEFSCHRIFT

ter verkrijging van de graad van doctor aan de Universiteit Maastricht, op gezag van de Rector Magnificus, Prof. dr. L.L.G. Soete

volgens het besluit van het College van Decanen, in het openbaar te verdedigen

op woensdag 1 juli 2015 om 12.00 uur

door

Johannes Buijsen 


\section{Promotor}

Prof. dr. Ph. Lambin

\section{Copromotores}

Dr. G. Lammering

Dr. M. Öllers

\section{Beoordelingscommissie}

Prof. dr. F.M. Mottaghy (voorzitter)

Dr. M. Lahaye

Prof. dr. C.A.M. Marijnen, Leids Universitair Medisch Centrum

Prof. dr. H.J.T. Rutten

Prof. dr. V.C.G. Tjan-Heijnen 
Voor mijn vader 



\section{Contents}

Chapter 1 Introduction

Part I: The role of PET-CT in tumor delineation

Chapter 2 FDG-PET provides the best correlation with the tumor specimen compared to MRI and CT

Chapter 3 FDG-PET-CT reduces the interobserver variability in rectal tumor delineation

\section{Part II: Response prediction in rectal cancer treatment}

Chapter 4 Development and external validation of a predictive model for pathological complete response of rectal cancer patients including sequential PET-CT imaging

Chapter 5 Blood biomarkers are helpful in the prediction of response to chemoradiation in rectal cancer: a prospective, hypothesis driven study on patients with locally advanced rectal cancer

Chapter 6 What biochemical and molecular biological factors have greater relevance to treatment decisions?

\section{Part III: Combined treatment in rectal cancer}

Chapter 7 Phase I trial of the combination of the Akt inhibitor nelfinavir and chemoradiation for locally advanced rectal cancer

Chapter 8 A phase I-II study on the combination of rapamycin and short course radiotherapy in rectal cancer

Chapter 9 General discussion and future perspectives

\section{Summary and valorization}

Chapter 10 Summary

Chapter 11 Valorization

\section{Appendix}

Dankwoord

Curriculum vitae 

Chapter 1

Introduction 
Colorectal cancer is a frequently diagnosed malignancy. Approximately 13,000 new cases are diagnosed in the Netherlands each year, which makes it the second most diagnosed malignancy after prostate cancer in men and breast cancer in women. Of these 13,000 colorectal tumors, 4,000 are located in the rectum. The incidence of rectal adenocarcinoma has risen from 2,400 new cases per year in 2001 to almost 4,000 in 2012 [1].

Over the past decades, the outcome of rectal cancer treatment has improved dramatically. Due to improvements in imaging technologies, surgical techniques and the increased use of radiotherapy, along with advances in knowledge about optimal timing of radiotherapy, locoregional recurrence rates have decreased from $25-30 \%$ in the seventies of the past century to less than $10 \%$, and in the majority of patients even less than $5 \%$ nowadays. With these high control rates, one may wonder whether there is still room for improvement of local treatment.

The most pronounced improvement in the treatment of rectal cancer came from the introduction of the concept of Total Mesorectal Excision (TME) by Heald [2]. The change from a classical blunt dissection to the sharp dissection led to a decrease in local failures of about 15\%. The Dutch TME trial showed that even with optimal surgical techniques short-course preoperative radiotherapy could halve the local recurrence rate [3]. Another important finding is the significance of the treatment sequence: preoperative radiotherapy has been proven superior to post-operative radiotherapy [4]. A British-Canadian group tested whether selective postoperative chemoradiation for patients with an involved circumferential resection margin (CRM) would have the same outcome as short-course ( $5 \times 5 \mathrm{~Gy}$ ) preoperative radiotherapy (SCRT) for all patients with a primary resectable rectal cancer. The local recurrence rate turned out to be higher in the postoperative group. Therefore, preoperative treatment remains the standard of care in rectal cancer treatment. A complicating factor of preoperative treatment is that decisions have to be based on clinical examination and imaging data, without knowing pathologic staging.

A more difficult question to answer is which preoperative treatment regimen should be chosen in which situation and if neo-adjuvant treatment can be 
omitted in a subgroup of patients. SCRT followed by immediate resection results in a $50 \%$ decrease of the chance of local recurrence in patients with primary resectable tumors. However, the absolute benefit depends on the risk of the individual patient and varied from $2.6 \%$ to $12 \%$ in the Dutch TME trial [5]. The absolute reduction of local recurrences is most pronounced in patients with pathological stage III disease $[5,6]$. In node negative patients with no or limited infiltration of the perirectal fat, pre-operative radiotherapy may be omitted. The prediction of nodal status remains a challenge, even in this era of rapidly developing imaging techniques. Until now, nodal staging is mainly based on size and morphological criteria. Although modern MRI scanners have a high spatial resolution, sensitivity and specificity remain low using these criteria [7, 8]. The use of special contrast agents can enhance the performance of MRI in nodal staging, but this technique is not widely available at this moment [9].

For locally advanced rectal cancer, defined as tumors with either a close relationship to the mesorectal fascia, invasion of surrounding organs or more than 3 positive lymph nodes, there is consensus that with the current evidence, chemoradiation is the treatment of choice [10], although some questions remain to be answered. One of these questions is whether the results of SCRT followed by surgery after an interval with or without systemic treatment are comparable to long course chemoradiation (CRT). Several trials are currently comparing these treatment options in a randomized setting. It is known that short course radiotherapy (SCRT) followed by an interval before surgery also leads to important downsizing [11-14]. The advantage of a treatment strategy with an interval of several weeks between radiotherapy and resection is that tumor downsizing can occur. This leads to a pathological complete response (pCR) in up to about $10 \%$ in patients treated with $5 \times 5$ Gy and resection after 6-8 weeks [11-14] and 15-20\% after CRT [15]. This group of patients seems to have a better prognosis [15], although it is not clear whether this PCR per se leads to the improved prognosis or whether it is merely a reflection of favorable tumor biology.

The observation that patients who develop a PCR have a better prognosis led to thoughts about ways to increase the chance of a pCR. An additional advantage 
of this approach is that more patients can be offered an organ sparing treatment. Although the omission of a surgical resection is still considered experimental, the first experiences in Brazil and in our own region are very promising [16-20]. Strategies to increase the response of tumors to radiotherapy include the administration of a higher dose to the tumor and the combination of radiotherapy with agents that make tumor cells more sensitive to radiation, so called radiosensitizers. It has indeed been shown that increasing the radiotherapy dose in rectal cancer increases the $\mathrm{pCR}$ rate [21-24]. The most used radiosensitizer in rectal cancer is 5-FU based chemotherapy. Combinations with classic forms of chemotherapy have been studied in phase II (5-FU + irinotecan) and phase III (5-FU + oxaliplatin) trials. This doublet chemotherapy led to an increase in toxicity without a significant increase in pathological response in the majority of trials [25-28], but the German CAO/ARO/AIO-04 trial found a modest but significant increase in $\mathrm{PCR}$ rate without a difference in toxicity [29]. Another approach is the combination of 5-FU based chemoradiation with new targeted drugs, like epidermal growth factor receptor (EGFR) antagonists and angiogenesis inhibitors. Until now, only small phase I and II trials have been published. Although preclinical data support the combination of these agents with neoadjuvant treatment, there is no evidence that response rates increase and some questions remain to be answered, like the optimal timing and the identification of patients who are going to benefit from the addition of a targeted agent to chemoradiation [30].

Although treatment results for rectal cancer have improved substantially, response to treatment is still very heterogeneous. In locally advanced rectal cancer treated with neoadjuvant chemoradiation, half of the patients do not show tumor down staging [31]. Predictive models may be helpful in choosing the right treatment approach for an individual patient. The advantage of these models is that they can include a wide range of prognostic and predictive factors, not only clinical, but also, for example, imaging and histological and molecular features [32]. As we learn more about molecular pathways that play a role in the development of rectal cancer and the response to treatment and the amount of imaging data from different modalities is increasing, it becomes 
more and more difficult for physicians to integrate all these factors and make the right treatment choice [33].

The aim of improving treatment response and tailoring treatment was the basis of the research presented in this thesis (figure 1 ).

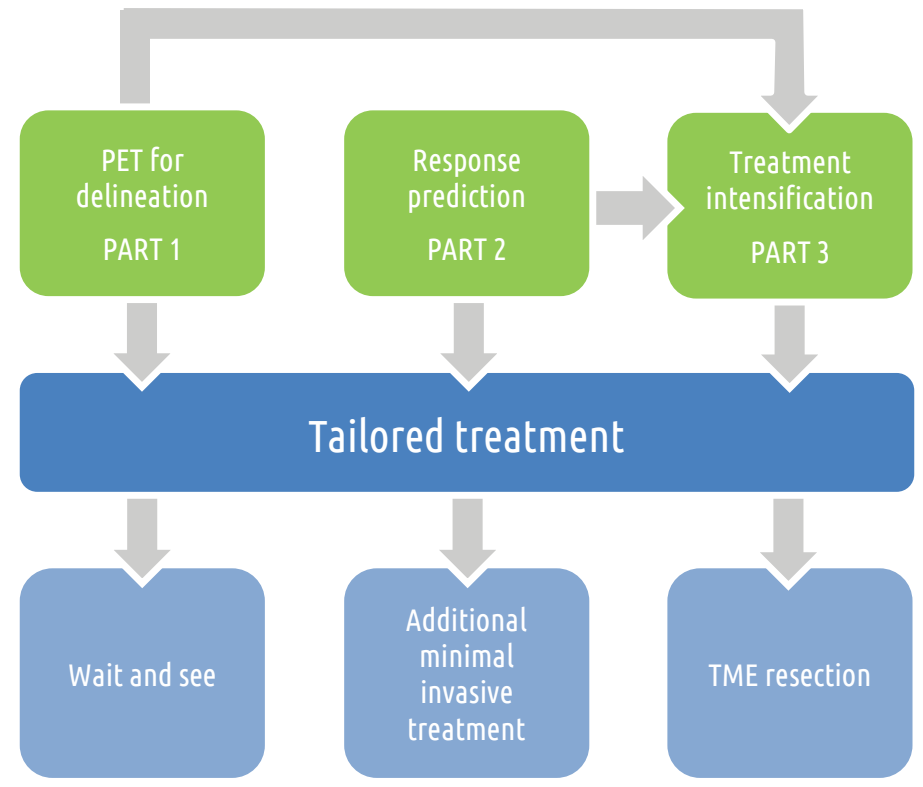

Figure 1 Schematic overview of the different topics discussed in this thesis and the treatment options for rectal cancer

\section{Outline of this thesis}

Three hypotheses formed the basis of this thesis:

1. PET-CT is a reliable tool for tumor delineation in rectal cancer treatment and diminishes interobserver variation.

2. Multimodality predictive models are able to predict response to neoadjuvant treatment in rectal cancer.

3. The combination of AKT inhibition and radiotherapy and mTOR inhibition and radiotherapy leads to an increased tumor response in rectal cancer. 
Studies related to the first hypothesis are presented in part I of this thesis. In chapter 2, we evaluated whether tumor dimensions in rectal cancers are adequately represented by the signal seen on a PET-scan. Chapter 3 describes the use of an automatic PET-based delineation algorithm and the influence on inter-observer variability in treatment volume definition.

The second hypothesis was the basis of part II of this thesis, which is dedicated to the role of different models for response prediction in rectal cancer. These models were developed to identify patients with a high chance of good response to neoadjuvant treatment. Chapter 4 describes a prediction model based on sequential PET-imaging before and during treatment. In chapter 5, the added value of blood biomarkers to the existing imaging based models is tested. Chapter 6 gives an overview of the literature on biochemical and molecular factors that predict response to treatment.

The third hypothesis is tested in two clinical trials presented in part III. These trials investigated the addition of a radiosensitizer to two different existing radiotherapy regimens in rectal cancer. In chapter 7 , the results of a phase I trial testing the combination of nelfinavir and chemoradiation are described and in chapter 8 a phase I-II study on the combination of the mTOR inhibitor rapamycin with short-course hypofractionated radiotherapy is reported.

Finally, in chapter 9, the different subjects of this thesis are discussed and recommendations for future research and clinical practice are given. 


\section{References}

[1] www.cijfersoverkanker.nl

[2] MacFarlane, JK, Ryall, RD, Heald, RJ. Mesorectal excision for rectal cancer. Lancet 1993;341:457-460.

[3] Kapiteijn, E, Marijnen, CA, Nagtegaal, ID, et al. Preoperative radiotherapy combined with total mesorectal excision for resectable rectal cancer. N Engl J Med 2001;345:638-646.

[4] Sauer, R, Becker, H, Hohenberger, W, et al. Preoperative versus postoperative chemoradiotherapy for rectal cancer. N Engl J Med 2004;351:1731-1740.

[5] van Gijn, W, Marijnen, CA, Nagtegaal, ID, et al. Preoperative radiotherapy combined with total mesorectal excision for resectable rectal cancer: 12-year follow-up of the multicentre, randomised controlled TME trial. Lancet Oncol 2011;12:575-582.

[6] Sebag-Montefiore, D, Stephens, RJ, Steele, R, et al. Preoperative radiotherapy versus selective postoperative chemoradiotherapy in patients with rectal cancer (MRC CR07 and NCIC-CTG C016): a multicentre, randomised trial. Lancet 2009;373:811-820.

[7] Bipat, S, Glas, AS, Slors, FJ, Zwinderman, AH, Bossuyt, PM, Stoker, J. Rectal cancer: local staging and assessment of lymph node involvement with endoluminal US, CT, and MR imaging--a meta-analysis. Radiology 2004;232:773-783.

[8] Brown, G, Richards, CJ, Bourne, MW, et al. Morphologic predictors of lymph node status in rectal cancer with use of high-spatial-resolution MR imaging with histopathologic comparison. Radiology 2003;227:371-377.

[9] Heijnen, LA, Lambregts, DM, Martens, MH, et al. Performance of gadofosveset-enhanced MRI for staging rectal cancer nodes: can the initial promising results be reproduced? Eur Radiol 2014;24:371-379.

[10] Valentini, V, Glimelius, B, Haustermans, K, et al. EURECCA consensus conference highlights about rectal cancer clinical management: the radiation oncologist's expert review. Radiother Oncol 2014;110:195-198.

[11] Hatfield, P, Hingorani, M, Radhakrishna, G, et al. Short-course radiotherapy, with elective delay prior to surgery, in patients with unresectable rectal cancer who have poor performance status or significant co-morbidity. Radiother Oncol 2009;92:210-214.

[12] Latkauskas, T, Pauzas, H, Gineikiene, I, et al. Initial results of a randomized controlled trial comparing clinical and pathological downstaging of rectal cancer after preoperative shortcourse radiotherapy or long-term chemoradiotherapy, both with delayed surgery. Colorectal Dis 2012;14:294-298.

[13] Pettersson, D, Holm, T, Iversen, H, Blomqvist, L, Glimelius, B, Martling, A. Preoperative shortcourse radiotherapy with delayed surgery in primary rectal cancer. Br J Surg 2012;99:577-583.

[14] Radu, C, Berglund, A, Pahlman, L, Glimelius, B. Short-course preoperative radiotherapy with delayed surgery in rectal cancer - a retrospective study. Radiother Oncol 2008;87:343-349. 
[15] Maas, M, Nelemans, PJ, Valentini, V, et al. Long-term outcome in patients with a pathological complete response after chemoradiation for rectal cancer: a pooled analysis of individual patient data. Lancet Oncol 2011;11:835-844.

[16] Habr-Gama, A, Perez, RO, Nadalin, W, et al. Operative versus nonoperative treatment for stage 0 distal rectal cancer following chemoradiation therapy: long-term results. Ann Surg 2004;240:711-717; discussion 717-718.

[17] Maas, M, Beets-Tan, RG, Lambregts, DM, et al. Wait-and-see policy for clinical complete responders after chemoradiation for rectal cancer. J Clin Oncol 2011;29:4633-4640.

[18] Habr-Gama, A, Sabbaga, J, Gama-Rodrigues, J, et al. Watch and wait approach following extended neoadjuvant chemoradiation for distal rectal cancer: are we getting closer to anal cancer management? Dis Colon Rectum 2013;56:1109-1117.

[19] Dalton, RS, Velineni, R, Osborne, ME, et al. A single-centre experience of chemoradiotherapy for rectal cancer: is there potential for nonoperative management? Colorectal Dis 2012;14:567-571.

[20] Glynne-Jones, R, Hughes, R. Critical appraisal of the 'wait and see' approach in rectal cancer for clinical complete responders after chemoradiation. Br J Surg 2012;99:897-909.

[21] Hernando-Requejo, O, Lopez, M, Cubillo, A, et al. Complete pathological responses in locally advanced rectal cancer after preoperative IMRT and integrated-boost chemoradiation. Strahlenther Onkol 2014;190:515-520.

[22] Burbach, JP, den Harder, AM, Intven, M, van Vulpen, M, Verkooijen, HM, Reerink, O. Impact of radiotherapy boost on pathological complete response in patients with locally advanced rectal cancer: A systematic review and meta-analysis. Radiother Oncol 2014.

[23] Appelt, AL, Ploen, J, Vogelius, IR, Bentzen, SM, Jakobsen, A. Radiation dose-response model for locally advanced rectal cancer after preoperative chemoradiation therapy. Int J Radiat Oncol Biol Phys 2013;85:74-80.

[24] Jakobsen, A, Ploen, J, Vuong, T, Appelt, A, Lindebjerg, J, Rafaelsen, SR. Dose-effect relationship in chemoradiotherapy for locally advanced rectal cancer: a randomized trial comparing two radiation doses. Int J Radiat Oncol Biol Phys 2012;84:949-954.

[25] Aschele, C, Cionini, L, Lonardi, S, et al. Primary tumor response to preoperative chemoradiation with or without oxaliplatin in locally advanced rectal cancer: pathologic results of the STAR-01 randomized phase III trial. J Clin Oncol 2011;29:2773-2780.

[26] Gerard, JP, Azria, D, Gourgou-Bourgade, S, et al. Clinical outcome of the ACCORD 12/0405 PRODIGE 2 randomized trial in rectal cancer. J Clin Oncol 2012;30:4558-4565.

[27] Lee, SU, Kim, DY, Kim, SY, et al. Comparison of two preoperative chemoradiotherapy regimens for locally advanced rectal cancer: capecitabine alone versus capecitabine plus irinotecan. Radiat Oncol 2013;8:258.

[28] Mohiuddin, M, Paulus, R, Mitchell, E, et al. Neoadjuvant chemoradiation for distal rectal cancer: 5-year updated results of a randomized phase 2 study of neoadjuvant combined 
modality chemoradiation for distal rectal cancer. Int J Radiat Oncol Biol Phys 2013;86:523-528.

[29] Rodel, C, Liersch, T, Becker, H, et al. Preoperative chemoradiotherapy and postoperative chemotherapy with fluorouracil and oxaliplatin versus fluorouracil alone in locally advanced rectal cancer: initial results of the German CAO/ARO/AIO-04 randomised phase 3 trial. Lancet Oncol 2012;13:679-687.

[30] Glynne-Jones, R, Hadaki, M, Harrison, M. The status of targeted agents in the setting of neoadjuvant radiation therapy in locally advanced rectal cancers. J Gastrointest Oncol 2013;4:264-284.

[31] Sloothaak, DA, Geijsen, DE, van Leersum, NJ, et al. Optimal time interval between neoadjuvant chemoradiotherapy and surgery for rectal cancer. Br J Surg 2013;100:933-939.

[32] Lambin, P, van Stiphout, RG, Starmans, MH, et al. Predicting outcomes in radiation oncology-multifactorial decision support systems. Nat Rev Clin Oncol 2013;10:27-40.

[33] Abernethy, AP, Etheredge, LM, Ganz, PA, et al. Rapid-learning system for cancer care. J Clin Oncol 2010;28:4268-4274. 
Chapter 1 
Part I

The role of PET-CT in tumor delineation

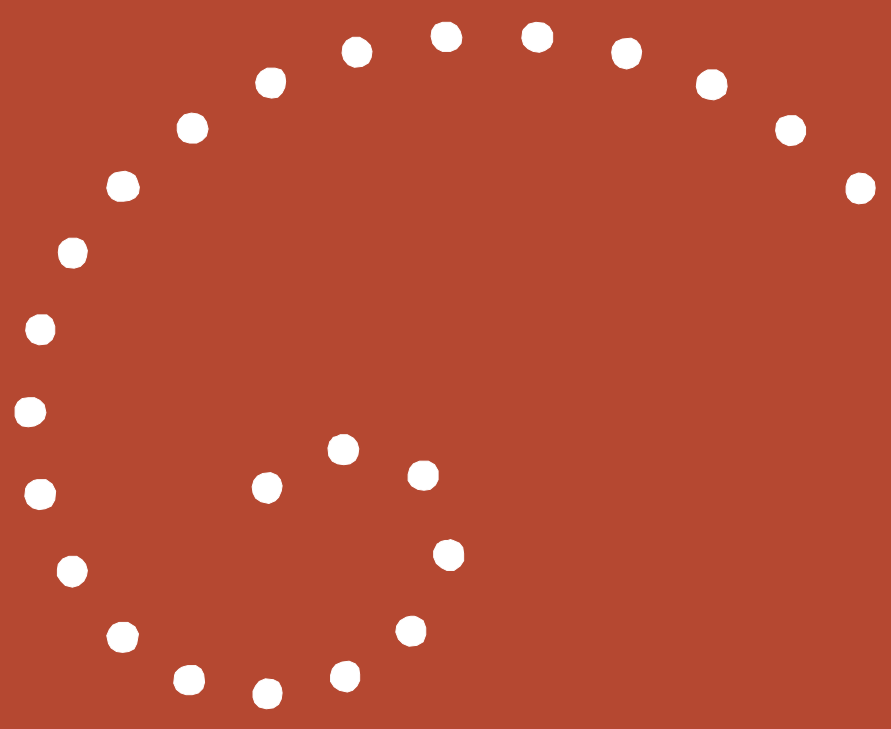





\section{Chapter 2 \\ FDG-PET provides the best correlation with the tumor specimen compared to $\mathrm{MRI}$ and $\mathrm{CT}$}

Jeroen Buijsen, Jørgen van den Bogaard, Marco H.M. Janssen, Frans C.H.

Bakers, Stephanie Engelsman, Michel Öllers, Regina G.H. Beets-Tan, Marius Nap, Geerard L. Beets, Philippe Lambin, Guido Lammering 


\section{Purpose}

To compare CT-, MR- and PET-CT based tumor length measurements in rectal cancer with pathology.

\section{Patients and Methods}

Twenty six rectal cancer patients underwent both MR and PET-CT imaging followed by short-course radiotherapy (RT 5x5 Gy) and surgery within 3 days after RT. Tumor length was measured manually and independently by 2 observers on CT, MR and PET. PETbased tumor length measurements were also generated automatically using the signalto-background-ratio (SBR) method. All measurements were correlated with the tumor length on the pathological specimen.

\section{Results}

CT-based measurements did not show a valuable correlation with pathology. MR-based measurements correlated only weakly, but still significantly (Pearson correlation $=0.55$ resp. $0.57 ; p<0.001$ ). Manual PET measurements reached a good correlation with pathology, but less strong (Pearson correlation 0.72 and 0.76 for the two different observers) than automatic PET-CT based measurements, which provided the best correlation with pathology (Pearson correlation of $0.91(p<0.001)$ ). Bland-Altman analysis demonstrated in general an overestimation of the tumor diameter using manual measurements, while the agreement of automatic contours and pathology was within acceptable ranges. A direct comparison of the different modalities revealed a significant better precision for PET-based auto-contours as compared to all other measurements.

\section{Conclusion}

Automatically generated PET-CT based contours show the best correlation with the surgical specimen and thus provide a useful and powerful tool to accurately determine the largest tumor dimension in rectal cancer. This could be used as a quick and reliable tool for target delineation in radiotherapy. However, a 3D volume analysis is needed to confirm these results. 


\section{Introduction}

Rectal cancer is a frequently occurring malignancy. Over the last two decades multimodality treatment has led to important improvements in the treatment of this disease. Preoperative RT, often combined with chemotherapy, followed by a Total Mesorectal Excision (TME) has become the standard of care for most patients. In this respect, modern imaging techniques are extremely important in the preoperative workup for treatment decision making. Endoscopic ultrasound (EUS) is most suitable for the evaluation of superficial tumors [1], whereas magnetic resonance (MR) imaging provides more accuracy in determining the margin between the tumor and the mesorectal fascia [2, 3].

While there is growing interest in the possible role of FDG-PET in response evaluation [4,5], until now, the use of 18F-fluoro-2-deoxy-glucose Positron Emission Tomography (FDG-PET) imaging has no clearly defined role in local staging of rectal cancer. However, a unique advantage of FDG-PET-scanning is the ability to use the quantitative information of the glucose uptake within the tumor to automatically create a contour around the tumor. This autocontouring process significantly reduces the interobserver variability in the interpretation of images $[6,7]$, as it eliminates the human factor, increases consistency, diminishes interobserver variability and also saves time. It is well known from other tumor sites that CT-based tumor delineations result in a substantial interobserver variation $[7,8]$. Since there is a growing interest in the use of a boost in the treatment of rectal cancer, accurate delineation of the tumor volume becomes increasingly important $[9,10]$. However, presently the evidence of the use of PET-CT in radiotherapy planning for rectal cancer is limited [11]. Therefore we need to compare this method with the pathological specimen, before we can use PET as a reliable method to define the Gross Tumor Volume (GTV) in rectal cancer. Thus, the aim of the present study was to evaluate the accuracy of FDG-PET-based auto-delineation in determining the maximal cranio-caudal diameter of rectal tumors and to compare it with $\mathrm{CT}$ and MR-based measurements. 


\section{Materials and methods}

Since the purpose of this study was to directly correlate the initial tumor length measurements with pathology as gold standard, we only included patients, who were referred for a short-course of 5 fractions of 5 Gy preoperative RT, followed by a TME within 3 days after the last fraction of radiotherapy. This preoperative treatment is known to have no influence on the size of the tumor if there is only a short time-window between completion of radiotherapy and resection [12]. In total, 26 patients diagnosed with primary resectable rectal cancer (T2-early T3, N0-1, with a predicted circumferential resection margin on MR of more than $2 \mathrm{~mm}$ ) were enrolled. As part of their preoperative workup, all patients had also been investigated with an MR scan.

\section{PET-CT imaging}

Before the start of radiotherapy, all patients underwent a PET-CT scan for treatment planning purposes using a PET-CT-simulator (Biograph, SOMATOM Sensation 16 with an integrated ECAT ACCEL PET scanner, Siemens, Knoxville, USA) with a spatial resolution of approximately $6.5 \mathrm{~mm}$ in the centre of the axial field of view (162 mm). Data acquisition was performed in 3D and the acquisition time per table position was 5 minutes. The acquired PET images were corrected for scatter and attenuation using a CT based attenuation map. Image reconstruction was performed using FORE (Fourier rebinning) and OSEM reconstruction (ordered subsets expectation maximization, 4 iterations, 8 subsets) with an inter-slice distance of $3.45 \mathrm{~mm}$. The patients were instructed to fast for at least 6 hours prior to PET-imaging. The FDG was injected intravenously, with the activity based on the patient weight (weight [kg] * $4+20$ [MBq]). PET-CT imaging was started approximately 60 minutes after FDG injection. The patient was scanned in supine position. At the start of the examination, a native CT scan was made from the top of the skull to the mid thigh. Scan parameters were $120 \mathrm{kV}, 140 \mathrm{~mA}$, a pitch of 1.25 and a $5 \mathrm{~mm}$ reconstructed section thickness. Immediately thereafter, a PET-scan was performed, covering the identical transverse field of view $(500 \mathrm{~mm})$. 


\section{Auto-delineation}

For study purposes, the tumor was automatically delineated using dedicated software (Esoft 5.0, Siemens MI, Erlangen, Germany). An SBR method was used to find for each patient a percentage threshold of the maximal SUV within a user defined Volume of Interest (VOI) around the tumor as previously described $[13,14]$. For each patient, the SBR was calculated, using the gluteus muscle as relevant background. From the SBR, the corresponding threshold percentage served as input for the SUV auto delineation algorithm in the Esoft software. The auto delineation algorithm is based on region growing [15]. Image voxels that are adjacent to one another in a certain neighborhood are clustered or connected.

Using this method, an auto-contour was created for each patient (figure 1) and the maximal cranio-caudal length of the contour was calculated automatically.

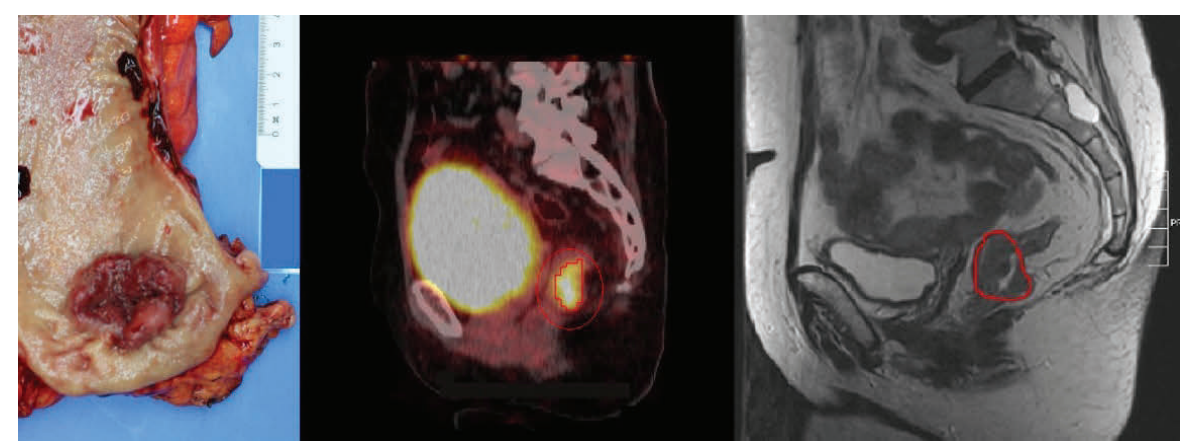

Figure 1 Images of respectively the surgical specimen, the corresponding CT-PET image and T2 weighted MR image of the same patient, with the tumor contour presented in red.

\section{Manual PET and CT measurements}

All PET-CT scans were imported in our treatment planning system (XiO/Focal 4.34.02, CMS Inc. Maryland Heights, MO, USA) and PET-scans were read by two radiation-oncologists with experience in the use of PET-CT for radiotherapy planning. A fixed window-level setting was used as well as standard color settings. Maximal tumor lengths were measured manually on a Focal workstation. At a second time point (at least 4 weeks after the PET- 
measurements to ensure that PET-measurements did not influence CTmeasurements), tumor lengths were measured by the same two radiationoncologists on the CT-images, without knowledge of MR and PET images.

\section{Magnetic Resonance (MR) imaging}

All patients underwent MR-imaging of the pelvis as a part of their preoperative workup. These scans were performed at the university hospital and in the surrounding referring hospitals. The field strength of the MR machines was 1 and $1.5 \mathrm{~T}$, and the protocol for rectal cancer MR imaging was regionally standardized, using T2W FSE sequences in 3 planes [16]. The maximum tumor length in cranio-caudal direction was measured manually on the PACS viewing system on the sagittal T2-weighted images, with the use of transversal and coronal projections when required. For this purpose the standard measurement tool in the viewing system was used. Measurements were performed independently by two experienced GI radiologists, blinded from any clinical information except for the distance from the anal verge to the caudal margin of the tumor on endoscopy and/or the distance to the tumor based on digital rectal examination.

\section{Pathology}

After resection, a standardized routine pathology examination was performed by a pathologist specialized in gastro-intestinal pathology using the protocol as described by Quirke et al [17]. The length of the tumor was carefully measured macroscopically with a ruler, before slicing was done. The pathologist was blinded from any information regarding the image-based tumor measurements.

\section{Statistical analysis}

SPSS $^{\circledR}$ software (SPSS ${ }^{\circledR}$ for Windows, version 15.0, SPSS Inc, Chicago, IL), was used to perform statistical analysis. Tumor diameters as measured with the different imaging modalities were compared with pathology using linear regression, including the Pearson correlation coefficient. In addition to correlation analysis, Bland-Altman analyses were performed to evaluate the 
absolute agreement of the measurements between the different modalities [18]. To directly compare the predictive performance of the various tests a method as described by Sheiner and Beal [19] was used. This method evaluates the predictive performance of a test by calculating the prediction error and the bias. The prediction error is an indicator for the precision of a test, while the bias gives information about the systematic component of the prediction error. In other words, the bias reflects an under- or overestimation of a test. Furthermore, this method gives the opportunity to compare the performance of the different imaging modalities.

\section{Results}

Tumor diameters varied from 1.6 to $8.0 \mathrm{~cm}$ on pathology examination (mean 4.2 $\mathrm{cm}$, SD 1.4), from 2.9 to $10.8 \mathrm{~cm}$ on $\mathrm{CT}$ (mean $5.1 \mathrm{~cm}$, SD $1.7 \mathrm{~cm}$ ) from 3.1 to 7.6 $\mathrm{cm}$ on MR (mean $4.8 \mathrm{~cm}$, SD 1.4) and from 2.2 to $8.1 \mathrm{~cm}$ on PET-scan (manual measurements, mean $5.0 \mathrm{~cm}$, SD $1.7 \mathrm{~cm}$ ) resp. 2.0 to $8.0 \mathrm{~cm}$ (automatic measurements, mean $4.2 \mathrm{~cm}$, SD 1.4). Individual measurements are shown in table 1.

The results of the Pearson correlation analyses are shown in figure 2. Measurement of the tumor length on CT showed no correlation with pathology for one observer and only a very weak correlation for the second observer (Pearson correlation $=0.34(p=0.09)$ resp. $0.50 \quad(p=0.01))$. Intra-observer correlation for CT-scan was the weakest of all modalities (Pearson correlation = $0.69 ; \mathrm{p}<0.001)$. The correlation between MR and pathology was somewhat stronger than $\mathrm{CT}$ for both observers (Pearson correlation $=0.55$ resp. 0.57; $p<0.001)$. Intra-observer correlation between both MR observers was less strong than for PET scan, but still considerable (Pearson correlation $=0.78(p<0.001)$ ). The correlation between manual PET measurements and pathology was stronger than for MR, resulting in a correlation coefficient of 0.76 for observer 1 and 0.72 for observer $2(p<0.001)$. Intra-observer correlation between the two PET-observers was very strong (Pearson correlation $=0.97, p<0.001)$. The best correlation was found between the automatic PET measurements and the 
measured tumor length on pathology (Pearson correlation $=0.91 ; p<0.001)$.

Table 1 Individual measurements of each modality in $\mathrm{cm}$.

\begin{tabular}{|c|c|c|c|c|c|c|c|c|}
\hline Patient & Pathology & $\mathrm{CT}_{\text {obs } 1}$ & $\mathrm{CT}_{\text {obs2 }}$ & $\mathrm{MRI}_{\mathrm{obs} 1}$ & MRI obs2 $_{\text {. }}$ & $\mathrm{PET}_{\operatorname{man} 1}$ & $\mathrm{PET}_{\text {man2 }}$ & $\mathrm{PET}_{\text {auto }}$ \\
\hline 1 & 6,0 & 5,8 & 5,8 & 6,9 & 8,2 & 6,8 & 6,6 & 4,8 \\
\hline 2 & 4,5 & 5,6 & 7,0 & 3,7 & 5,7 & 5,5 & 6,3 & 5,0 \\
\hline 3 & 4,0 & 4,0 & 3,2 & 3,4 & 2,8 & 4,2 & 4,0 & 3,8 \\
\hline 4 & 8,0 & 8,0 & 9,0 & 7,3 & 7,3 & 7,8 & 8,1 & 8,0 \\
\hline 5 & 3,0 & 4,0 & 3,5 & 3,5 & 3,2 & 3,9 & 4,3 & 3,0 \\
\hline 6 & 4,0 & 4,3 & 4,2 & 4,4 & 4,2 & 5,0 & 4,9 & 4,2 \\
\hline 7 & 2,9 & 4,3 & 3,8 & 3,9 & 3,7 & 5,6 & 5,4 & 3,2 \\
\hline 8 & 5,0 & 6,0 & 5,7 & 4,1 & 5,5 & 6,2 & 6,4 & 5,2 \\
\hline 9 & 4,0 & 4,6 & 5,8 & 5,4 & 4,6 & 4,3 & 3,7 & 3,7 \\
\hline 10 & 3,5 & 3,5 & 2,2 & 3,6 & 4,8 & 3,8 & 2,9 & 3,0 \\
\hline 11 & 4,5 & 6,3 & 4,3 & 4,8 & 4,3 & 6,6 & 6,5 & 4,8 \\
\hline 12 & 5,5 & 7,3 & 6,2 & 4,3 & 3,5 & 8,2 & 7,7 & 7,0 \\
\hline 13 & 4,0 & 5,5 & 4,8 & 4,7 & 4,7 & 6,0 & 5,8 & 4,9 \\
\hline 14 & 4,0 & 4,0 & 4,3 & 6,4 & 6,5 & 7,4 & 7,5 & 4,5 \\
\hline 15 & 5,0 & 7,1 & 6,2 & 6,3 & 4,8 & 6,6 & 6,3 & 5,7 \\
\hline 16 & 3,0 & 4,6 & 1,6 & 3,2 & 3,6 & 3,0 & 2,2 & 2,0 \\
\hline 17 & 3,0 & 4,5 & 3,2 & 2,5 & 3,7 & 2,6 & 3,0 & 3,0 \\
\hline 18 & 6,0 & 5,0 & 4,4 & 5,5 & 5,1 & 6,8 & 6,4 & 6,5 \\
\hline 19 & 4,2 & 3,9 & 4,5 & 4,5 & 3,8 & 3,2 & 3,0 & 3,6 \\
\hline 20 & 3,5 & 12,4 & 9,1 & 6,9 & 7,1 & 5,4 & 6,0 & 4,5 \\
\hline 21 & 3,2 & 6,1 & 1,8 & 3,9 & 3,4 & 4,2 & 3,9 & 2,8 \\
\hline 22 & 3,0 & 3,6 & 5,3 & 3,1 & 3,0 & 2,8 & 3,2 & 2,9 \\
\hline 23 & 1,6 & 4,5 & 3,9 & 3,6 & 3,4 & 3,1 & 3,5 & 2,4 \\
\hline 24 & 3,8 & 4,7 & 5,3 & 5,9 & 4,3 & 4,2 & 3,8 & 4,0 \\
\hline 25 & 4,0 & 6,3 & 5,0 & 6,7 & 4,5 & 4,4 & 4,2 & 4,1 \\
\hline 26 & 3,8 & 7,2 & 4,4 & 7,1 & 7,0 & 4,0 & 3,9 & 3,6 \\
\hline
\end{tabular}



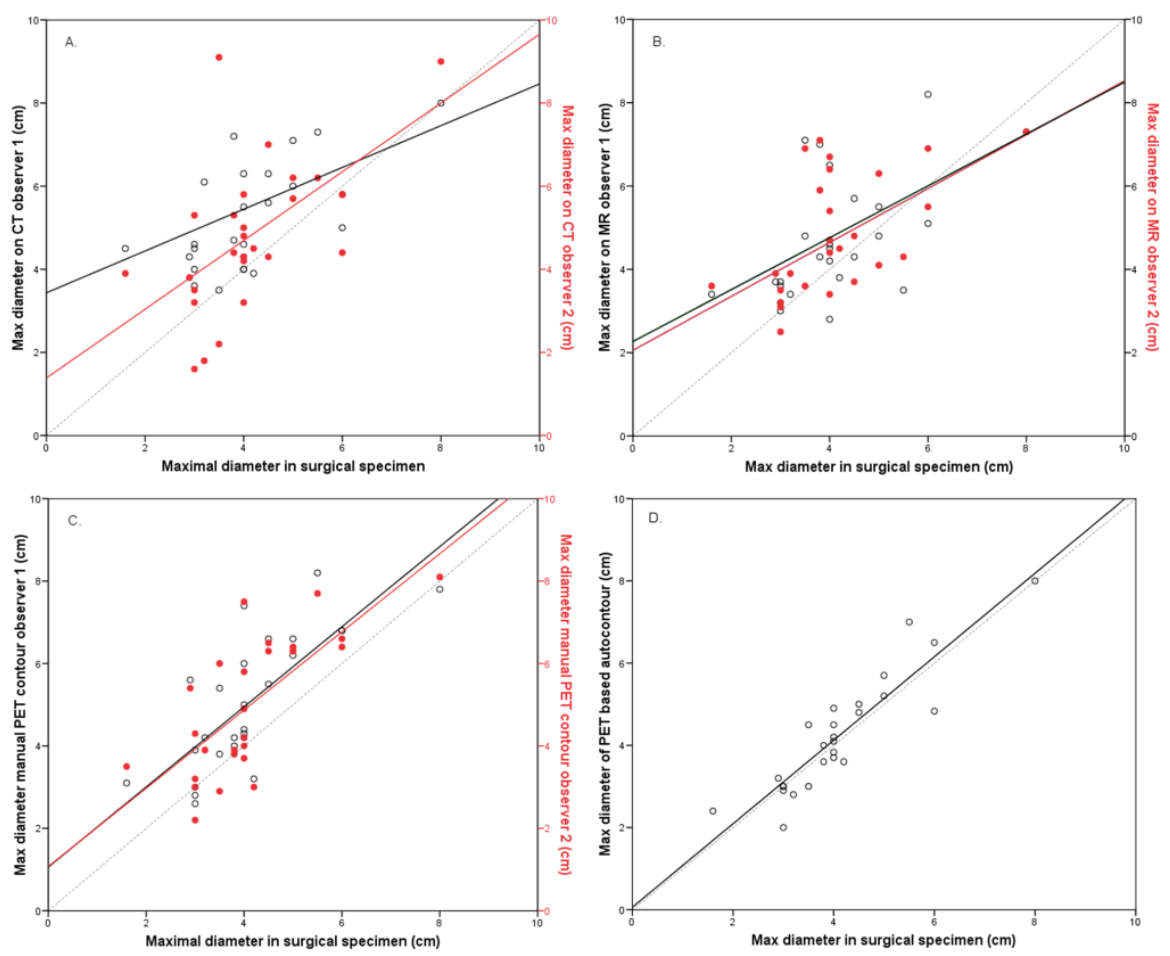

Figure 2 Linear regression curves for maximal tumor diameter resulting from CT-imaging (2 observers, red and black) (A), MR-imaging (2 observers) (B), manual PET-contours (2 observers) (C) and automatic PET-contours (D). The dotted line represents the ideal situation of absolute agreement between two modalities.

A comparative analysis using Bland-Altman plots (figure 3) shows that CT scans tend to overestimate tumor length, reflected by a mean difference of 1.03 with limits of agreement -1.99 and 4.04 . This means that $95 \%$ of the diameters measured on CT lie within a range of -1.99 to $+4.04 \mathrm{~cm}$ from the true diameter as measured in the pathology specimen. The agreement between MR and pathology proved to be better than CT but worse than automatic PETmeasurements: the mean difference reached $0.66 \mathrm{~cm}$ (limits of agreement -1.69 and 3.01). For manual measurements on PET scan, differences were somewhat larger (mean difference 0.91, limits of agreement -1.23 and 3.05) than for automatically generated contours on PET scans, which showed the best 
agreement with pathology. For the majority of patients the agreement between PET auto-contours and pathology was within $1 \mathrm{~cm}$. The mean difference was $0.13 \mathrm{~cm}$ and the limits of agreement were -1.06 and 1.31 .
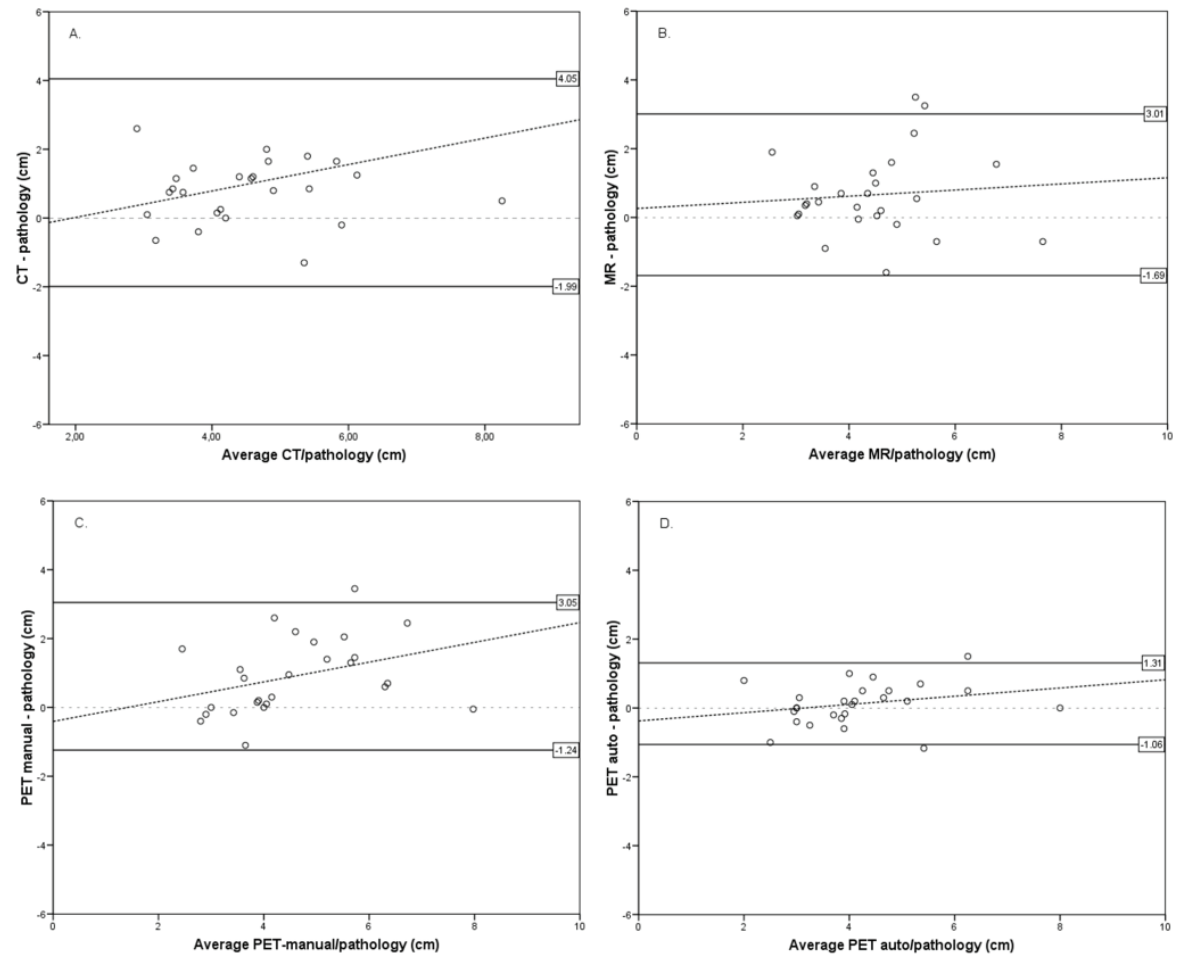

Figure 3 Bland-Altman plots showing the difference against mean for the three imaging modalities (A. CT, B. MR, C. manual PET contours, D. automatic PET contours). The thick lines represent the confidence intervals around the mean of difference, marking the level of agreement between the different imaging modalities.

The results of the analysis of precision and bias are shown in table 2. These results are in line with the observations in the Bland-Altman plots: PETautocontours show the highest precision and the smallest bias and this precision was significantly better than any other measurement in this study, as can be gathered from the $95 \%$ confidence intervals. 
Table 2 Predictive performance of the different modalities using the method as described by Sheiner and Beal[19].

\begin{tabular}{llllll}
\hline & PET auto & PET manual & MR & CT & Endoscopy \\
\hline Precision & 0.37 & 1.97 & 1.82 & 3.35 & 3.82 \\
Bias & 0.13 & 0.91 & 0.66 & 1.03 & 0.46 \\
Difference in & & -1.60 & -1.45 & -2.98 & -3.45 \\
precision & & $(-2.71--0.50)$ & $(-2.70--0.19)$ & $(-4.00--1.96)$ & $(-7.60-0.70)$ \\
$(95 \% \mathrm{Cl})^{1}$ & & & & & \\
\hline
\end{tabular}

${ }^{1}$ Difference as compared to the performance of PET auto-contour

\section{Discussion}

This is the first study demonstrating a strong correlation between the rectal tumor length measured in an automated way on PET-images and pathology as reference. These automated PET-based tumor measurements showed a stronger correlation with pathology than CT, MR and manual PET measurements.

PET-based auto delineation and correlation with pathology in rectal cancer has been described in one other study [20]. This study found only a modest correlation with pathology. However, these patients were treated with a long course of chemoradiotherapy, which certainly resulted in heterogeneous tumor downsizing, thus strongly influencing the interpretation of correlative studies between pre-treatment images and pathology. Furthermore, a different delineation algorithm was used. Our findings are in concordance with the paper of Van Baardwijk et al. and Daisne et al. who found that source-to-background ratio-based auto-delineation showed a good correlation with pathology in respectively lung and head and neck cancer [7, 21]. Daisne et al. compared FDGPET with MR and CT and found PET to have the strongest correlation with pathology in pharyngeal and laryngeal cancer [21]. However, it should be mentioned that their results were based on a relatively small group of 12 patients. An advantage of their study is that they were able to make a reconstruction of the tumor volume. Unfortunately, we were unable to reliably 
measure the 3D tumor volume in the surgical specimen. However, we made the assumption that the tumor length is indicative for the volume, as has been shown before for cervical cancer [22]. For future research we have planned to develop a method for accurate volume measurements. A possible limitation of our study is that the macroscopic tumor diameters may have been measured at different time points after resection. This may have led to different degrees of shrinkage of the surgical specimen. No conclusive data on tumor shrinkage has been reported for rectal cancer. However, in prostate cancer, linear correction factors for tumor shrinkage between 1.04 and 1.14 have been described [23], indicating that the influence of shrinkage might be rather low. Since the composition of the tumor stroma of colorectal tumors in general is even more compact than the fibromuscular stroma of the prostate, we assume that the shrinkage effect in colorectal tumors by formalin will be even less than in the prostate.

Another characteristic of our patient group is that they all have received shortcourse radiotherapy pre-operatively. In the Netherlands, pre-operative radiotherapy has become standard of care for the large majority of rectal cancer patients. Therefore it was for ethical reasons not feasible to include patients who would only undergo surgery. As mentioned earlier no downstaging has been observed in the Dutch TME trial after 5x5 Gy. However, they observed a small but statistically significant difference in tumor diameter between the irradiated and the non-irradiated group ( 4.5 vs $4.0 \mathrm{~cm}, \mathrm{p}<0.001)$. They did not report on reliable tumor measurements at diagnosis, making it impossible to draw firm conclusions whether this difference in tumor size may have been caused by tumor shrinkage as a result of radiotherapy. In addition, a substantial part of the patient group in the TME trial (57\%) was operated 4 or 5 days after completion of radiotherapy, while in our patient group all patients were operated within 3 days. However, in order to have more certainty that tumor shrinkage did not influence our results, we went on and analyzed the maximal diameters on PET-CT scans of 21 rectal cancer patients who had been imaged with PET-CT before and immediately after the fifth fraction of radiotherapy as part of a sequential PET-CT study [4]. The mean tumor diameters as measured 
by auto-contouring were 5.1 vs $5.0 \mathrm{~cm}$ on pre- vs. post-radiotherapy scans $(p=0.13)$. This to our opinion confirms that at least up until day 5 of radiotherapy no significant tumor shrinkage has taken place. It is rather unlikely that 3 further days at the time of surgery would still result in a measurable downsizing.

For tumors with smaller volumes it is important to rule out the influence of partial volume effects (PVE) on PET-imaging [24]. PVE typically occur if the tumor size is smaller than three times the spatial resolution (that means $3 \times 6.5$ $\mathrm{mm}=1.95 \mathrm{~cm}$ ). As the smallest maximal tumor diameter in this patient group as measured on PET-scan was $2 \mathrm{~cm}$, we do not expect that PVE had an important influence on the data. Due to the limited spatial resolution of PET-imaging, one may not expect PET-scan to be able to detect microscopic tumor extension.

For the current radiotherapy treatment planning of rectal cancer, PET-imaging is only of limited value in the determination of the radiation treatment field borders, because the preoperative treatment of rectal cancer normally includes a locoregional treatment encompassing the whole posterior pelvis. However, new developments of intensified regimens aiming at completely eradicating the tumor might lead to the necessity of an accurate definition of the tumor volume in order to avoid geographical misses and to limit the volume of normal tissue being irradiated. So far, several groups studied the use of PET-imaging for the delineation of rectal tumors. Patel et al. found in a limited number of patients $(n=6)$, that the use of PET-CT in radiotherapy planning for rectal cancer resulted in a decrease in the inter-observer variability in boost target volumes [6]. Bassi et al. recently reported an important increase in the target volume when using PET-CT data as compared to only CT [25]. Anderson et al showed a slight decrease in the delineated volume based on PET-CT as compared to CT. The mean overlap volume between the CT-based tumor volume and PET-based tumor volume was 46.7\% [26]. Very recently, the group of Paskeviciute described the impact of FDG-PET-scan on planning of neoadjuvant radiotherapy in rectal cancer [27]. They also found a decrease in volumes defined on basis of PET information. However, their $\mathrm{CT}$ volume consisted of the entire rectal circumference at the level of the tumor, whereas the PET volume consisted of 
the PET positive part of the rectal wall only with a $2 \mathrm{~cm}$ margin. They found geographical misses in $46 \%$ of the patients. The use of MR scans when delineating on CT is certainly helpful and is expected to diminish differences between contouring on $\mathrm{CT}$ only and PET-CT. Another important difference between our study and these two other studies is that they did not use automatic delineation software.

A disadvantage of the use of PET-imaging in colorectal cancer is the fact that PET has only a very low sensitivity for nodal disease [28]. For that reason PET-CT is not suitable for sub-boosting of pathological nodes. Furthermore, PET-CT cannot reliably predict the involvement of the mesorectal fascia [29]. Therefore MR certainly has an important role in decision making in rectal cancer. Another disadvantage of PET-scan is the low spatial resolution. Therefore, it is difficult to compare the inter-observer variabilities between the different imaging modalities found in this study. It cannot be entirely ruled out that the better agreement between the two observers on PET-scan is partially the result of the lower spatial resolution of PET as compared to CT and MRI.

As mentioned before, the use of auto-contours avoids interobserver differences [7]. Furthermore, it is well known that manual delineation is largely operatordependent [30]. Our own group previously compared delineation on CT with auto-contours generated on PET-scans using static and dynamic PET-scans in rectal cancer patients [31]. We found that manually drawn contours on CT were significantly larger than contours based on dynamic PET-scans. In this cohort, no direct comparison was made between static PET-scan contours using the SBR method and manual contours.

In conclusion, this is, to our knowledge, the first study that confirms the accuracy of PET-CT-based tumor length measurements in rectal cancer by pathology validation. PET-CT-based measurements show a strong correlation with pathology compared to other frequently used imaging modalities and can therefore be defined as a useful tool for the GTV delineation in rectal cancer. 


\section{References}

[1] Glancy, DG, Pullyblank, AM, Thomas, MG. The role of colonoscopic endoanal ultrasound scanning (EUS) in selecting patients suitable for resection by transanal endoscopic microsurgery (TEM). Colorectal Dis 2005;7:148-150.

[2] Diagnostic accuracy of preoperative magnetic resonance imaging in predicting curative resection of rectal cancer: prospective observational study. BMJ 2006;333:779.

[3] Beets-Tan, RG, Beets, GL, Vliegen, RF, et al. Accuracy of magnetic resonance imaging in prediction of tumor-free resection margin in rectal cancer surgery.Lancet 2001;357:497-504.

[4] Janssen, MH, Ollers, MC, van Stiphout, RG, et al. Evaluation of early metabolic responses in rectal cancer during combined radiochemotherapy or radiotherapy alone: sequential FDGPET-CT findings. Radiother Oncol 2010;94:151-155.

[5] Mak, D, Joon, DL, Chao, M, et al. The use of PET in assessing tumor response after neoadjuvant chemoradiation for rectal cancer. Radiother Oncol 2010, doi:10.1016/j.radonc.2010.05.016

[6] Patel, DA, Chang, ST, Goodman, KA, et al. Impact of integrated PET/CT on variability of target volume delineation in rectal cancer. Technol Cancer Res Treat 2007;6:31-36.

[7] van Baardwijk, A, Bosmans, G, Boersma, L, et al. PET-CT-based auto-contouring in non-smallcell lung cancer correlates with pathology and reduces interobserver variability in the delineation of the primary tumor and involved nodal volumes. Int J Radiat Oncol Biol Phys 2007;68:771-778.

[8] Leunens, G, Menten, J, Weltens, C, Verstraete, J, van der Schueren, E. Quality assessment of medical decision making in radiation oncology: variability in target volume delineation for brain tumors. Radiother Oncol 1993;29:169-175.

[9] Seierstad, T, Hole, KH, Saelen, E, Ree, AH, Flatmark, K, Malinen, E. MR-guided simultaneous integrated boost in preoperative radiotherapy of locally advanced rectal cancer following neoadjuvant chemotherapy. Radiother Oncol 2009;93:279-284.

[10] Krishnan, S, Janjan, NA, Skibber, JM, et al. Phase II study of capecitabine (Xeloda) and concomitant boost radiotherapy in patients with locally advanced rectal cancer. Int J Radiat Oncol Biol Phys 2006;66:762-771.

[11] Lambrecht, M, Haustermans, K. Clinical evidence on PET-CT for radiation therapy planning in gastro-intestinal tumors. Radiother Oncol 2010;96:339-346.

[12] Marijnen, CA, Nagtegaal, ID, Klein Kranenbarg, E, et al. No downstaging after short-term preoperative radiotherapy in rectal cancer patients. J Clin Oncol 2001;19:1976-1984.

[13] Daisne, JF, Sibomana, M, Bol, A, Doumont, T, Lonneux, M, Gregoire, V. Tri-dimensional automatic segmentation of PET volumes based on measured source-to-background ratios: influence of reconstruction algorithms. Radiother Oncol 2003;69:247-250. 
[14] Ollers, M, Bosmans, G, van Baardwijk, A, et al. The integration of PET-CT scans from different hospitals into radiotherapy treatment planning. Radiother Oncol 2008;87:142-146.

[15] Pratt, WK. Digital Image Processing: PIKS Scientific Inside. Los Altos California: John Wiley\&Sons. 2007.

[16] Beets-Tan, RG, Beets, GL. Rectal cancer: review with emphasis on MR imaging. Radiology 2004;232:335-346.

[17] Quirke, P, Durdey, P, Dixon, MF, Williams, NS. Local recurrence of rectal adenocarcinoma due to inadequate surgical resection. Histopathological study of lateral tumor spread and surgical excision. Lancet 1986;2:996-999.

[18] Bland, JM, Altman, DG. Statistical methods for assessing agreement between two methods of clinical measurement. Lancet 1986;1:307-310.

[19] Sheiner, LB, Beal, SL. Some suggestions for measuring predictive performance. J Pharmacokinet Biopharm 1981;9:503-512.

[20] Ciernik, IF, Huser, M, Burger, C, Davis, JB, Szekely, G. Automated functional image-guided radiation treatment planning for rectal cancer. Int J Radiat Oncol Biol Phys 2005;62:893-900.

[21] Daisne, JF, Duprez, T, Weynand, B, et al. Tumor volume in pharyngolaryngeal squamous cell carcinoma: comparison at $\mathrm{CT}$, MR imaging, and FDG PET and validation with surgical specimen. Radiology 2004;233:93-100.

[22] Jung, DC, Ju, W, Choi, HJ, et al. The validity of tumor diameter assessed by magnetic resonance imaging and gross specimen with regard to tumor volume in cervical cancer patients. Eur J Cancer 2008;44:1524-1528.

[23] Jonmarker, S, Valdman, A, Lindberg, A, Hellstrom, M, Egevad, L. Tissue shrinkage after fixation with formalin injection of prostatectomy specimens. Virchows Arch 2006;449:297301.

[24] Soret, M, Bacharach, SL, Buvat, I. Partial-volume effect in PET tumor imaging. J Nucl Med 2007;48:932-945.

[25] Bassi, MC, Turri, L, Sacchetti, G, et al. FDG-PET/CT imaging for staging and target volume delineation in preoperative conformal radiotherapy of rectal cancer. Int J Radiat Oncol Biol Phys 2008;70:1423-1426.

[26] Anderson, C, Koshy, M, Staley, C, et al. PET-CT fusion in radiation management of patients with anorectal tumors. Int J Radiat Oncol Biol Phys 2007;69:155-162.

[27] Paskeviciute, B, Bölling, T, Brinkmann, M, et al. Impact of 18F-FDG-PET/CT on staging and irradiation of patients with locally advanced rectal cancer. Strahlenther Onkol 2009;185: 260-265.

[28] Kantorova, I, Lipska, L, Belohlavek, O, Visokai, V, Trubac, M, Schneiderova, M. Routine (18)FFDG PET preoperative staging of colorectal cancer: comparison with conventional staging and its impact on treatment decision making. J Nucl Med 2003;44:1784-1788. 
[29] Vliegen, RF, Beets-Tan, RG, Vanhauten, B, et al. Can an FDG-PET/CT predict tumor clearance of the mesorectal fascia after preoperative chemoradiation of locally advanced rectal cancer? Strahlenther Onkol 2008;184:457-464.

[30] Lee, JA. Segmentation of positron emission tomography images: some recommendations for target delineation in radiation oncology. Radiother Oncol 2010;96:302-307.

[31] Janssen, MH, Aerts, HJ, Ollers, MC, et al. Tumor delineation based on time-activity curve differences assessed with dynamic fluorodeoxyglucose positron emission tomographycomputed tomography in rectal cancer patients. Int J Radiat Oncol Biol Phys 2009;73:456-465. 
Chapter 2 


\section{Chapter 3 \\ FDG-PET-CT reduces the interobserver variability in rectal tumor delineation}

Jeroen Buijsen, Jørgen van den Bogaard, Hiske van der Weide, Stephanie Engelsman, Ruud van Stiphout, Marco Janssen, Geerard Beets, Regina BeetsTan, Philippe Lambin, Guido Lammering 


\section{Background and purpose}

Previously, we showed a good correlation between pathology and an automatically generated PET-contour in rectal cancer. This study analysed the effect of the use of PETCT scan on the interobserver variation in GTV definition in rectal cancer and the influence of PET-CT on treatment volumes.

\section{Materials and methods}

42 patients diagnosed with rectal cancer underwent an FDG-PET-CT for radiotherapy planning. An automatic contour was created on PET-scan using the source-tobackground ratio. The GTV was delineated by 5 observers in 3 rounds: using CT and MRI, using CT, MRI and PET and using CT, MRI and PET auto-contour. GTV volumes were compared and concordance indices ( $\mathrm{Cl})$ were calculated. Since the GTV is only a small portion of the treatment volume in rectal cancer, a separate analysis was performed to evaluate the influence of PET on the definition of the CTV used in daily clinical practice and the caudal extension of the treatment volumes.

\section{Results}

GTV volumes based on PET were significantly smaller. Cls increased significantly using PET and the best interobserver agreement was observed using PET auto-contours. Furthermore, we found that in that in up to $29 \%$ of patients the CTV based on PET extended outside the CTV used in clinical practice. The caudal border of the treatment volume can be tailored using PET-scan in low seated tumors. Influence of PET on the position of the caudal border was most pronounced in low seated tumors.

\section{Conclusion}

PET-CT increases the interobserver agreement in the GTV definition in rectal cancer, helps to avoid the geographical misses and allows tailoring the caudal border of the treatment volume. 


\section{Introduction}

Pre-operative radiotherapy has become an essential part of the treatment of most patients with rectal cancer, since it is very effective in reducing the risk of a locoregional recurrence [1]. Combining radiotherapy and chemotherapy results in downsizing and in up to $10-33 \%$ of patients pathological complete responses ( $\mathrm{pCR}$ ) have been reported [2-5]. Patients with a good clinical response may benefit from less invasive surgery, like sphincter-saving surgery or transanal endoscopic microsurgery (TEM). Even, in selected cases, a wait-and-see policy might be safe [6]. These innovative modified surgical approaches may lead to better quality of life. Thus, it would be attractive to further increase the probability of a good tumor response, e.g. by increasing the dose to the tumor $[5,7,8]$. To achieve higher doses to the tumor, a simultaneous integrated boost technique has been shown to be feasible [9-11]. In order to identify the boost volume easily and reliably, high quality imaging is important. MRI is considered the most accurate staging method for rectal cancer [12-14], but its role in a precise determination of the boost volume is unknown [15]. For PET-imaging it has been reported that it is reliable in defining tumor size in rectal cancer [16] and has the additional advantage that it can easily be acquired in treatment position simultaneously with a CT-scan, which is needed for treatment planning.

Another reason why it is important to define more precisely the primary tumor in rectal cancer is that it may help to reduce long term toxicity that is observed after radiotherapy for rectal cancer $[17,18]$, through a further reduction of treatment fields. As our group has shown before, 3-D conformal planning results in a better PTV coverage and dose homogeneity as compared to standard 3- or 4-field techniques based on bony anatomy [19]. However, the use of more conformal techniques, like IMRT, poses the risk of geographical misses. Furthermore individual delineation makes it possible to better spare normal tissues. A better identification of the primary tumor allows avoiding irradiation of the sphincter in selected cases and reducing the volume of small bowel in high seated tumors, resulting in less toxicity. It has been shown that positive 
lymph nodes are most frequently located at the level of the tumor. The proximal spread of lymph nodes is limited to $5 \mathrm{~cm}$ from the distal margin of the tumor and the distance to the most distal nodes is $4 \mathrm{~cm}$ at maximum $[20,21]$. Furthermore, an analysis of the Dutch TME trial showed that in primary resectable rectal cancer short course radiotherapy is especially effective in the prevention of anastomotic recurrences [22,23]. This finding confirms that it is important to know the exact location of the tumor in order to safely reduce radiotherapy treatment fields.

For these reasons we hypothesized that the use PET-CT can help to define the GTV in rectal cancer more accurate leading to a better tailored definition of the treatment volume, that it would diminish interobserver variability and diminish the time needed to define the GTV. Furthermore we hypothesized that the influence of the use of PET-CT on treatment volume would be larger in low seated tumors as compared to high rectal tumors, because of the low soft tissue contrast on CT in the lower part of the pelvis.

\section{Methods and materials}

For this study 42 patients diagnosed with rectal cancer (cT2-4N0-2M0) were selected. Patients were scheduled to undergo a neo-adjuvant treatment consisting of chemoradiotherapy (28x1.8 Gy with concurrent capecitabine 825 $\mathrm{mg} / \mathrm{m} 2$ bid). All patients underwent an FDG-PET-CT scan for radiotherapy planning on an integrated PET-CT scanner (Truepoint Biograph 40, Siemens Erlangen, Germany). The PET-scan protocol has been described in detail earlier [24]. PET-CT images were fused and an automatic contour around the primary tumor was created, using the Signal-to-Background-Ratio (SBR)-method as described earlier $[25,26]$ using dedicated software (Esoft 5.0, Siemens MI, Erlangen, Germany). This contouring method has been shown to have a good correlation with pathology in rectal cancer [16].

We balanced the number of patients with low- and high-seated tumors ( 22 high, 20 low). For this study high seated tumors had a caudal border $\geq 7 \mathrm{~cm}$ from the anal verge. 
For delineation, fixed window/level settings were used (400/50 for CT and $30000 / 15000$ for PET). In PET-scans not showing enough contrast using these settings, an adjusted W/L setting was used, identical for all 5 observers.

\section{GTV delineation and interobserver variability}

The GTV was delineated by 5 observers: 2 radiation-oncologists sub-specialized in gastro-intestinal (GI) tumors, 1 senior-resident, 1 radiation-technologist and 1 radiologist. At the time of delineation clinical details were available and presented in a standardized format to each observer including the findings on digital rectal examination and the endoscopy and pelvic MR-imaging reports.

Each study set was delineated 3 times by each observer in 3 consecutive rounds. During each delineation round observers had, in addition to the standardized clinical information, access to different imaging information, creating 3 sets of GTV contours per observer. In each round an MR-scan was available. MR and CT were not fused and MR was projected on a second screen. Round 1: MR- and CTimages only (CT-GTV (GTVCT)), round 2: MR-, CT- and PET-images (PET-GTV (GTVPET)) and in round 3 in addition to the MR-, CT- and PET-images the automatic generated contour on PET was provided (automatic GTV (GTVauto)). In the third round observers were asked to edit the provided contour, in such a way to obtain a clinically acceptable GTV. For each observer, the delineation rounds were spaced with a minimum interval of 4 weeks, to prevent bias from a preceding delineation round. Observers were blinded to each other's delineations.

Time needed for each delineation was registered by the observers. The volumes of the different GTVs were collected from the planning system and compared. Pairs of contours from the 3 different delineation methods were compared by calculating the concordance index $(\mathrm{Cl})$, defined as the ratio of the intersection and the union of the two volumes $[27,28]$.

$$
C I=\frac{(A \cap B)}{(A \cup B)}
$$


Differences in GTV delineation were analysed for the total patient group as well as for high- and low-seated tumors separately.

\section{CTV delineation and interobserver variability}

Since the GTV is only a small part of the clinical target volume in the current treatment of rectal cancer, 3 observers also delineated the complete CTV as used in daily clinical practice ( $\mathrm{CTV}_{\text {compl }}$ ), including regional lymph nodes, according to our local protocol as described earlier [19]. In brief, the CTV included at least $3 \mathrm{~cm}$ of the rectal wall in the oral and aboral direction, to cover possible intramural tumor spread, the mesorectal subsite, posterior pelvic subsite, and the regional lymph nodes at risk, which were defined by contouring the internal iliac vessels with a margin of $5 \mathrm{~mm}$ and the obturator region for low seated tumors ( $<7 \mathrm{~cm}$ from the anal verge in this protocol). The obturator region was delineated as proposed by Roels et al [29]. The CTV of the primary tumor was obtained by circumferential expansion of the GTVs with $0.5 \mathrm{~cm}$, resulting in $\mathrm{CTV}_{\mathrm{CT}}, \mathrm{CTV}_{\mathrm{PET}}$ and $\mathrm{CTV}_{\text {auto. }}$. The percentages of the different CTVs located outside the $\mathrm{CTV}_{\text {compl }}$ were analysed.

We were particularly interested in the caudal tumor extension. There is evidence that the risk of microscopic disease in lymph nodes $>4 \mathrm{~cm}$ caudal from the caudal border of the primary tumor is very limited [20,21]. Reducing the caudal CTV extension could result in a reduced radiation dose delivered to the anal sphincter, decreasing the risk of sphincter dysfunction as described in patients undergoing pre-operative radiotherapy followed by sphincter sparing surgery $[18,30]$. Therefore we also analyzed if the caudal border intramural margin differed between the CT- and PET-based delineations.

\section{Statistics}

SPSS 17.0 (SPSS, Chicago, IL) was used to perform statistical analysis. For the comparison of the differences in time needed to perform the delineations a paired-samples t-test was used. The volumes of the GTVs, Cls of both methods, as well as the percentages of CTV lying outside the CTV $\mathrm{Compl}_{\text {land }}$ anderences in caudal borders were compared using the Wilcoxon signed rank test, because 
data did not follow a normal distribution. Two-sided $\mathrm{p}$-values are provided; $\mathrm{p}$ values $<0.05$ were considered significant.

\section{Results}

The availability of PET images resulted in about $40 \%$ decrease in time needed to complete GTV delineation (mean time GTV ${ }_{\text {CT }} 4.1 \mathrm{~min}, \mathrm{GTV}_{\text {PET }} 2.5 \mathrm{~min}(\mathrm{p}<.001$ ) and GTV auto $1.6 \min (\mathrm{p}<.001))$.

The volumes for each observer are shown in table 1. GTV volumes were significantly smaller using PET-scan (mean GTV $_{\mathrm{CT}} 46.8 \mathrm{~cm}^{3}$ vs. mean GTV PET $\left.28.8 \mathrm{~cm}^{3}(p<0.001)\right)$. Editing automatically created contours resulted in the smallest volumes (mean GTV auto $18.2 \mathrm{~cm}^{3}(\mathrm{p}<.001)$ ).

Table 1 Mean GTV volumes for the 5 different observers using three different delineation methods.

\begin{tabular}{|c|c|c|c|c|c|c|c|c|c|c|c|c|}
\hline \multirow{2}{*}{ 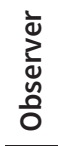 } & \multicolumn{3}{|c|}{ CT-based } & \multicolumn{3}{|c|}{ PET-based } & \multicolumn{4}{|c|}{ PET-auto } & \multirow[b]{2}{*}{$\mathrm{p}^{1}$} & \multirow[b]{2}{*}{$p^{2}$} \\
\hline & Mean & Range & & Mean & Range & & $\mathrm{p}$ & Mean & Range & & & \\
\hline All & 46.8 & 6.3 & 185.7 & 28.8 & 1.5 & 131.9 & $<0.001$ & 23.6 & 2.4 & 96.3 & $<0.001$ & $<0,001$ \\
\hline 1 & 41.2 & 6.3 & 159.3 & 26.0 & 1.5 & 107.3 & $<0.001$ & 23.3 & 2.4 & 88.1 & $<0.001$ & 0,27 \\
\hline 2 & 52.7 & 11.6 & 168.5 & 26.2 & 3.8 & 115.3 & $<0.001$ & 23.0 & 2.8 & 87.5 & $<0.001$ & 0,24 \\
\hline 3 & 48.8 & 9.6 & 185.7 & 37.2 & 7.7 & 131.9 & $<0.001$ & 25.3 & 2.5 & 93.6 & $<0.001$ & $<0,001$ \\
\hline 4 & 43.6 & 7.3 & 166.6 & 29.6 & 2.6 & 119.7 & $<0.001$ & 23.6 & 2.7 & 89.7 & $<0.001$ & $<0,001$ \\
\hline 5 & 47.4 & 7.9 & 167.5 & 25.2 & 4.5 & 128.7 & $<0.001$ & 22.9 & 2.5 & 89.9 & $<0.001$ & 0,21 \\
\hline
\end{tabular}

An example of the delineation of 2 patients is depicted in figure 1 . Conformity indices increased when PET information was added, reflecting a better agreement between observers. The mean conformity index (mean \pm SD) for the 5 observers was $0.79 \pm 0.17$ (range: $0-0.98$ ) using CT only in combination with MRI, $0.82 \pm 0.16$ (range: $0.10-1.00, \mathrm{p}=0.103$ ) using PET-data without automatically created contours and $0.93 \pm 10.5$ (range: $0.28-1.00, \mathrm{p}<0.001$ ) using PET auto- 
contours (figure 2). Using CT-scans only, in 2 cases a complete disagreement between observers occurred (reflected by a $\mathrm{Cl}$ of 0 ).

No differences were found between low- and high-seated tumors: 0.78 vs 0.79 $(p=0.31)$ for CT-only, 0.82 vs. $0.82(p=0.50)$ for PET-manual and 0.93 vs 0.92 $(p=0.94)$ for PET with auto-contours (figure 2$)$.
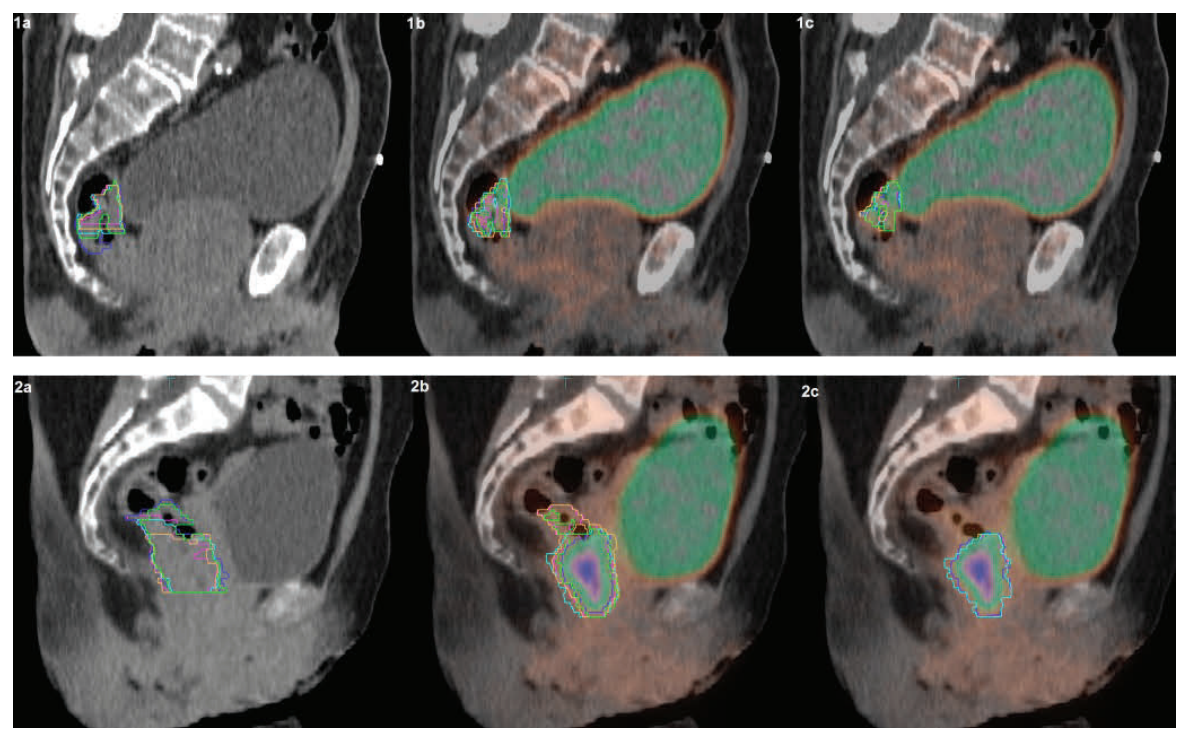

Figure 1 Example of the delineations by 5 observers in a patient with a high-seated (1) and a lowseated tumor (2), based on CT-only (a), PET-CT (b) and PET-CT with auto-contour (c).

The analysis of the CTVs showed that, with the addition of PET, in some patients a part of the tumor CTV was not covered by the $\mathrm{CTV}_{\text {compl. }}$. The mean $\mathrm{Cl}$ for CTV $\mathrm{V}_{\text {PET }}$ was 0.98 (range: $0.27-1.00$ ) and for CTV $_{\text {auto }} 0.98$ (0.29-1.00). For CTV PET the percentage of volume lying outside CTV $_{\text {compl }}$ exceeded $5 \%$ in 8 cases (19\%) (4 times in observer 1, 2 times in observer 2 and 2 times in observer 3). For CTV auto this was the case in 12 delineations (29\%) (6 times in observer 1, 3 times in observer 2 and 2 times in observer 3). In 1 patient more than $75 \%$ of the CTV PET and $\mathrm{CTV}_{\text {auto }}$ was lying outside the $\mathrm{CTV}_{\text {compl }}$ in 2 observers. This was a high seated tumor which was not correctly delineated based on CT and MR only, but was correctly identified on PET. 
On average, the caudal border of the intramural margin was located $0.6 \mathrm{~cm}$ more cranial if based on automatic PET-contours as compared to CT. In 7 patients (17\%), the caudal border of the PET-based margin was located $1 \mathrm{~cm}$ or more caudal than the CT-based margin for at least one observer. Four of them had a high seated tumor, 3 had a low seated tumor. In 3 of these patients the intramural margin extended $\geq 1 \mathrm{~cm}$ caudally based on PET for all 3 observers. In $19(45 \%)$ patients the caudal border of the PET-margin was located $\geq 1 \mathrm{~cm}$ higher than the CT-margin. In 6 patients this difference of $\geq 1 \mathrm{~cm}$ was observed in all 3 observers, in another 6 patients in 2 observers and in the remaining 7 patients in 1 observer. Sixteen of these tumors were located high and 3 were located low. On average the caudal border of PET-margin of high-seated tumors was located $1.1 \mathrm{~cm}$ more cranial than CT-margin. For low seated tumors the difference was negligible (figure 3). The difference between low- and highseated tumors was statistically significant.

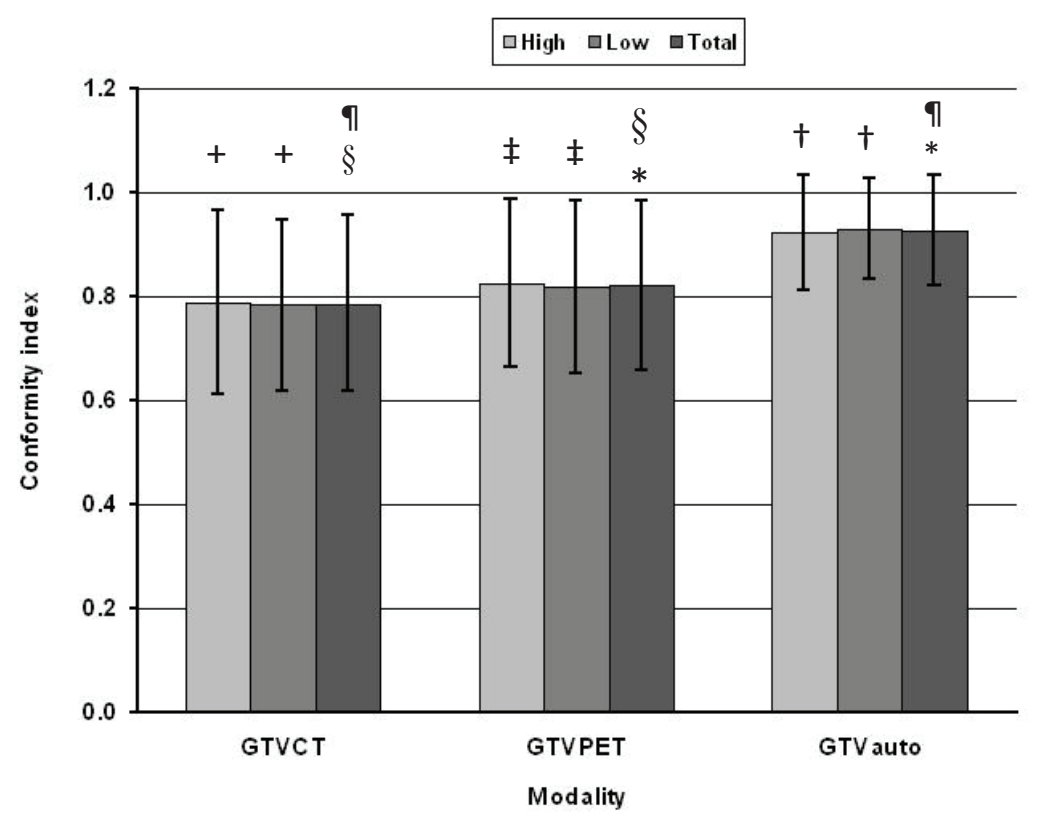

Figure 2 Concordance index according to delineation method (CT, PET-CT and PET-CT with autocontour) and divided in low seated ( $\leq 7 \mathrm{~cm}$ from the anal verge) and high seated tumors. + : $p=0.31, \ddagger: p=0.50, \dagger: p=0.94, \S: p=0.103$, ฯ: $p<0.001,{ }^{\star}: p<0.001$ 


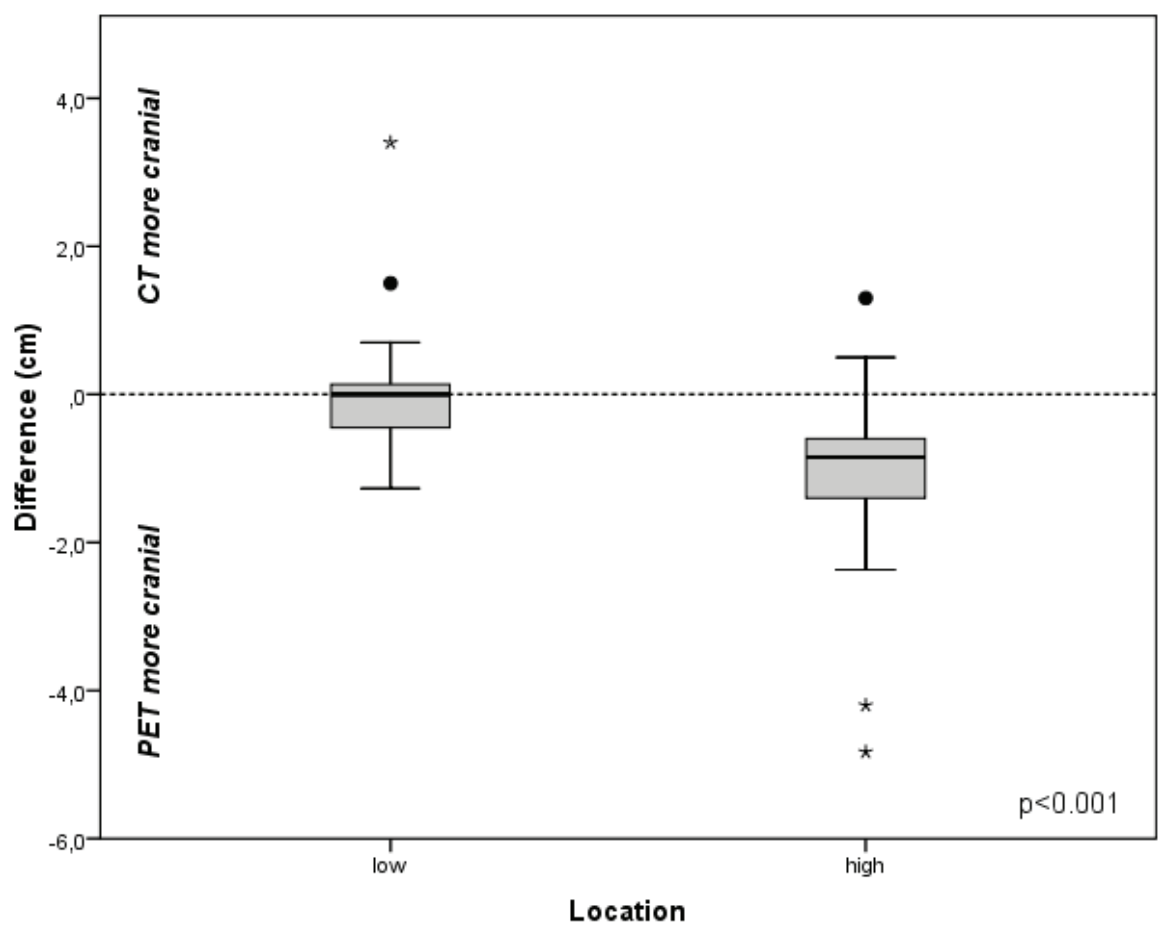

Figure 3 Mean difference \pm SD in $\mathrm{cm}$ of the 3 observers who delineated the complete treatment volume between the most caudal extension of the intramural margin based on CT and the caudal boundary based on the automatic PET-based contour. A negative value means that the caudal border based on PET was located more cranial than the CT-based border (i.e. in that case the volume based on PET was shorter in caudal direction).

\section{Discussion}

To the best of our knowledge, this is the first study looking at the influence of the use of automatically created PET-contours on GTV delineation and interobserver variability in rectal cancer. As has been shown earlier, the SBR method results in contours with a very good correlation with pathology [16]. The smallest GTV volumes are created using PET-based autocontours as compared to CT-based contours and manual PET-based contours (23.6 cc vs. $28.8 \mathrm{cc}$ vs. $46.9 \mathrm{cc}$ ). This study confirms that the use of PET-based autocontours leads to a very good interobserver agreement, reflected by a mean $\mathrm{Cl}$ of 0.93 for 
automatic PET-contours as compared to 0.82 (manual PET-contours) and 0.79 (CT-based contours). Furthermore, we found that PET-scan may help to avoid geographical misses in selected cases, especially in very low and very high located tumors and therefore may be helpful to define an adequate boost volume. In addition it could help to reduce treatment fields leading to a reduction of the amount of sphincter in the radiation volume, possibly leading to less late sphincter related toxicity and it allows for GTV-boosting in dose escalation trials.

A better interobserver agreement using PET-scan has been shown for the delineation of lung-, brain- and head and neck tumors [31-33]. Furthermore it has been demonstrated that the use of automatically created contours results in better agreement than visual interpretation of PET [34]. Obviously, visual interpretation depends on many factors, like window/level settings and the display mode of the PET information (for example grayscale or colormode) and experience of the observers. In this study 5 observers with different experience and background were asked to delineate the GTVs. Although it is difficult to draw firm conclusions, we observed that the differences in volumes differed statistically significant for all but 2 observer combinations when using CT data only. Differences in volume became smaller using PET-data and Cls decreased for all observers, indicating that PET is helpful for observers with different levels of experience in delineation of rectal tumors.

Other groups have looked at the influence of PET on target volume delineation before. Ciernik et al [35] was the first group that published about the use of PET$\mathrm{CT}$ in delineation for rectal cancer using a growing region algorithm. They found a good correlation with GTVs created manually on $\mathrm{CT}$, but did not analyse the influence of PET information on delineation decisions by the physician. No comparison was made between different observers. In a study of Bassi et al [36] tumor delineation was done by 2 radiation-oncologists together. They concluded that GTVs based on PET were significantly smaller than CT-based GTVs, which is in line with our findings. Patel et al compared delineations of tumor and lymph nodes in 6 rectal cancer patients who underwent an FDG-PET 
as well as FLT-PET [37]. In contrast to our findings, they did not observe clear differences in GTV volumes, but the interobserver agreement was better using PET. No differences between FDG-PET and FLT-PET were seen and no SUVbased auto-contour was used.

Results of rectal cancer treatment have improved markedly in the last decades, due to better surgical techniques and the widespread use of radiotherapy [1]. However, the use of radiotherapy results in long-term toxicity in a substantial part of patients. Therefore, it is important to make a better patient selection on one hand and to tailor treatment fields as much as possible on the other hand. Irradiation of the sphincter may result in problems with fecal continence [38, 39]. In the Dutch TME trial $62 \%$ of patients without a stoma reported faecal incontinence or soiling in the radiotherapy and surgery arm versus 38\% in the surgery only arm [18]. This study shows that the use of PET-scan can help to better tailor treatment fields. Especially the caudal border of the radiation fields can be limited in an important proportion of patients, resulting in a lower sphincter dose and a lower dose to the distal rectal wall in higher seated tumors, without the risk of geographical misses. Theoretically this could lead to less late toxicity. We hypothesized that the influence of PET on GTV volume and caudal extension of the treatment volume would be largest in lower seated tumors. This study showed that influence of PET was not different between lowand high-seated tumors. Therefore, the use of PET may be beneficial to all rectal cancer patients. When looking specifically at the influence of imaging modality on the position of the caudal border of the treatment volume (figure 3), it can be concluded that PET is helpful to individualize this border. In some patients this border will be located more cranial when based on PET, possibly resulting in a lower dose to the sphincter and distal rectal wall, while in others it will be located more distal. For the analysis of the caudal border we included a $3 \mathrm{~cm}$ margin in the course of the rectal wall in both directions. In our protocol we do not stop this margin at the border of the sphincter. For example: if the caudal border of the tumor is located $2 \mathrm{~cm}$ cranial to the sphincter, $1 \mathrm{~cm}$ of the anal canal was included in the intramural margin. It can be argued whether this is 
really necessary or whether one could see the anorectal junction as an anatomical barrier. Of course this can influence the results of our analysis. Recent literature suggests that distal surgical margins as close as $1 \mathrm{~cm}$ may be safe, but this is based on surgical data of patients who have been treated with pre-operative radiotherapy in majority $[40,41]$. Therefore, we do not feel comfortable at the moment to leave the complete sphincter out of the treatment volume in very low lying tumors and use the intramural margin based on PET-scan.

As stated in the introduction, adequate identification of the tumor is essential to create a reliable boost volume. This allows studying if a boost to the primary tumor results in more pathological complete responses and if this can lead to the use of less invasive surgery. This study shows that use of PET results in a good agreement between observers and our pathology validation study showed a very strong agreement between tumor length defined by automatic PETcontours and measured by the pathologist in the surgical specimen. If we assume that the representation of the position of the tumor and tumor edges is accurate, we can conclude that PET-CT makes it possible to define a reliable GTV in rectal cancer. Although we think that this assumption is very plausible, no analysis of the position of the tumor on PET-CT and in vivo has been performed. Therefore, in clinical practice this method should be used with caution and a clinical prospective evaluation is necessary.

A second problem that has to be solved to define an adequate boost volume is the internal organ motion, which can be quite substantial in the case of rectal cancer. It has been shown that especially in the cranial part of the mesorectum deformations can be quite substantial and that these deformations are caused mainly by differences in bowel filling [42].

Although a PET-scan adequately images the primary tumor and can be used for tumor delineation, it is not reliable for the distinction between benign and pathological lymph nodes [43]. The specificity is acceptable, but the sensitivity is rather low [44-46]. Therefore, additional imaging is strongly needed to 
adequately identify positive nodes. MR in combination with special contrast agents seems to be a promising method [47]. For this study we did not compare different PET segmentation algorithms, because we found a good correlation between pathology and SBR-based PET-contours. However, the SBR-method has several disadvantages [48]. It is dependent on many parameters, so that each modification in the process makes it necessary to perform a new calibration and each scanner has to be calibrated separately. In addition this method does not perform well if the source-to-background ratio is low. In future projects we will compare the performance of other segmentation methods with the SBR method in rectal cancer. Apart from a useful tool in delineation, FDGPET can also be helpful to get insight in tumor heterogeneity. Our group has shown that in NSCLC residual metabolic active areas after radiotherapy are the areas with the highest uptake before treatment $[49,50]$. Recently we showed that this is also the case for rectal cancer [51]. Another step for the future could therefore be the development of sub-boosting techniques.

In conclusion, PET-CT reduces interobserver variation and volumes in GTV definition in rectal cancer, enables tailoring treatment fields, especially in cranio-caudal direction, and makes it possible to define volumes for boosting. The use of PET-CT makes it possible to create a reliable boost volume in the treatment of rectal cancer and is expected to reduce toxicity due to the tailoring of treatment fields. 


\section{References}

[1] Valentini, V, Beets-Tan, R, Borras, JM, et al. Evidence and research in rectal cancer. Radiother Oncol 2008;87:449-474.

[2] Balch, GC, De Meo, A, Guillem, JG. Modern management of rectal cancer: a 2006 update. World J Gastroenterol 2006;12:3186-3195.

[3] Kim, DW, Huamani, J, Fu, A, Hallahan, DE. Molecular strategies targeting the host component of cancer to enhance tumor response to radiation therapy. Int $\mathrm{J}$ Radiat Oncol Biol Phys 2006;64:38-46.

[4] Minsky, BD, Cohen, AM, Kemeny, N, et al. Enhancement of radiation-induced downstaging of rectal cancer by fluorouracil and high-dose leucovorin chemotherapy. J Clin Oncol 1992;10:79-84.

[5] Wiltshire, KL, Ward, IG, Swallow, C, et al. Preoperative radiation with concurrent chemotherapy for resectable rectal cancer: effect of dose escalation on pathologic complete response, local recurrence-free survival, disease-free survival, and overall survival. Int J Radiat Oncol Biol Phys 2006;64:709-716.

[6] Habr-Gama, A, Perez, RO, Proscurshim, I, et al. Patterns of failure and survival for nonoperative treatment of stage c0 distal rectal cancer following neoadjuvant chemoradiation therapy. J Gastrointest Surg 2006;10:1319-1328.

[7] Chan, AK, Wong, AO, Langevin, J, et al. Preoperative chemotherapy and pelvic radiation for tethered or fixed rectal cancer: a phase II dose escalation study. Int J Radiat Oncol Biol Phys 2000;48:843-856.

[8] Mohiuddin, M, Regine, WF, John, WJ, et al. Preoperative chemoradiation in fixed distal rectal cancer: dose time factors for pathological complete response. Int J Radiat Oncol Biol Phys 2000;46:883-888.

[9] Seierstad, T, Hole, KH, Saelen, E, Ree, AH, Flatmark, K, Malinen, E. MR-guided simultaneous integrated boost in preoperative radiotherapy of locally advanced rectal cancer following neoadjuvant chemotherapy. Radiother Oncol 2009;93:279-284.

[10] De Ridder, M, Tournel, K, Van Nieuwenhove, Y, et al. Phase II study of preoperative helical tomotherapy for rectal cancer. Int J Radiat Oncol Biol Phys 2008;70:728-734.

[11] Guerrero Urbano, MT, Henrys, AJ, Adams, EJ, et al. Intensity-modulated radiotherapy in patients with locally advanced rectal cancer reduces volume of bowel treated to high dose levels. Int J Radiat Oncol Biol Phys 2006;65:907-916.

[12] Beets-Tan, RG, Beets, GL, Vliegen, RF, et al. Accuracy of magnetic resonance imaging in prediction of tumor-free resection margin in rectal cancer surgery. Lancet 2001;357:497-504.

[13] Beets-Tan, RG, Beets, GL. Rectal cancer: how accurate can imaging predict the T stage and the circumferential resection margin? Int J Colorectal Dis 2003;18:385-391. 
[14] MERCURY, SG. Diagnostic accuracy of preoperative magnetic resonance imaging in predicting curative resection of rectal cancer: prospective observational study. BMJ 2006;333:779.

[15] Lambrecht, M, Haustermans, K. Clinical evidence on PET-CT for radiation therapy planning in gastro-intestinal tumors. Radiother Oncol 2010;96:339-346.

[16] Buijsen, J, van den Bogaard, J, Janssen, MH, et al. FDG-PET provides the best correlation with the tumor specimen compared to MRI and CT in rectal cancer. Radiother Oncol 2011;98:270276.

[17] Marijnen, CA, van de Velde, CJ, Putter, H, et al. Impact of short-term preoperative radiotherapy on health-related quality of life and sexual functioning in primary rectal cancer: report of a multicenter randomized trial. J Clin Oncol 2005;23:1847-1858.

[18] Peeters, KC, van de Velde, CJ, Leer, JW, et al. Late side effects of short-course preoperative radiotherapy combined with total mesorectal excision for rectal cancer: increased bowel dysfunction in irradiated patients--a Dutch colorectal cancer group study. J Clin Oncol 2005;23:6199-6206.

[19] Borger, JH, van den Bogaard, J, de Haas, DF, et al. Evaluation of three different CT simulation and planning procedures for the preoperative irradiation of operable rectal cancer. Radiother Oncol 2008;87:350-356.

[20] Hida, J, Yasutomi, M, Maruyama, T, Fujimoto, K, Uchida, T, Okuno, K. Lymph node metastases detected in the mesorectum distal to carcinoma of the rectum by the clearing method: justification of total mesorectal excision. J Am Coll Surg 1997;184:584-588.

[21] Koh, DM, Brown, G, Temple, L, et al. Distribution of mesorectal lymph nodes in rectal cancer: in vivo MR imaging compared with histopathological examination. Initial observations. Eur Radiol 2005;15:1650-1657.

[22] Kusters, M, Marijnen, CA, van de Velde, $\mathrm{CJ}$, et al. Patterns of local recurrence in rectal cancer; a study of the Dutch TME trial. Eur J Surg Oncol 2010;36:470-476.

[23] Nijkamp, J, Kusters, M, Beets-Tan, RG, et al. Three-dimensional Analysis of Recurrence Patterns in Rectal Cancer: The Cranial Border in Hypofractionated Preoperative Radiotherapy Can Be Lowered. Int J Radiat Oncol Biol Phys 2011;80:103-110.

[24] Janssen, MH, Aerts, HJ, Ollers, MC, et al. Tumor delineation based on time-activity curve differences assessed with dynamic fluorodeoxyglucose positron emission tomographycomputed tomography in rectal cancer patients. Int J Radiat Oncol Biol Phys 2009;73:456465.

[25] Daisne, JF, Sibomana, M, Bol, A, Doumont, T, Lonneux, M, Gregoire, V. Tri-dimensional automatic segmentation of PET volumes based on measured source-to-background ratios: influence of reconstruction algorithms. Radiother Oncol 2003;69:247-250.

[26] Ollers, M, Bosmans, G, van Baardwijk, A, et al. The integration of PET-CT scans from different hospitals into radiotherapy treatment planning. Radiother Oncol 2008;87:142-146. 
[27] Giraud, P, Elles, S, Helfre, S, et al. Conformal radiotherapy for lung cancer: different delineation of the gross tumor volume (GTV) by radiologists and radiation oncologists. Radiother Oncol 2002;62:27-36.

[28] van Baardwijk, A, Bosmans, G, Boersma, L, et al. PET-CT-based auto-contouring in non-smallcell lung cancer correlates with pathology and reduces interobserver variability in the delineation of the primary tumor and involved nodal volumes. Int J Radiat Oncol Biol Phys 2007;68:771-778.

[29] Roels, S, Duthoy, W, Haustermans, K, et al. Definition and delineation of the clinical target volume for rectal cancer. Int J Radiat Oncol Biol Phys 2006;65:1129-1142.

[30] Stephens, RJ, Thompson, LC, Quirke, P, et al. Impact of short-course preoperative radiotherapy for rectal cancer on patients' quality of life: data from the Medical Research Council CR07/National Cancer Institute of Canada Clinical Trials Group C016 randomized clinical trial. J Clin Oncol 2010;28:4233-4239.

[31] De Ruysscher, D, Kirsch, CM. PET scans in radiotherapy planning of lung cancer. Radiother Oncol 2010;96:335-338.

[32] Grosu, AL, Weber, WA. PET for radiation treatment planning of brain tumors. Radiother Oncol 2010;96:325-327.

[33] Troost, EG, Schinagl, DA, Bussink, J, Oyen, WJ, Kaanders, JH. Clinical evidence on PET-CT for radiation therapy planning in head and neck tumors. Radiother Oncol 2010;96:328-334.

[34] Lee, JA. Segmentation of positron emission tomography images: some recommendations for target delineation in radiation oncology. Radiother Oncol 2010;96:302-307.

[35] Ciernik, IF, Huser, M, Burger, C, Davis, JB, Szekely, G. Automated functional image-guided radiation treatment planning for rectal cancer. Int J Radiat Oncol Biol Phys 2005;62:893-900.

[36] Bassi, MC, Turri, L, Sacchetti, G, et al. FDG-PET/CT imaging for staging and target volume delineation in preoperative conformal radiotherapy of rectal cancer. Int J Radiat Oncol Biol Phys 2008;70:1423-1426.

[37] Patel, DA, Chang, ST, Goodman, KA, et al. Impact of integrated PET/CT on variability of target volume delineation in rectal cancer. Technol Cancer Res Treat 2007;6:31-36.

[38] al-Abany, M, Helgason, AR, Cronqvist, AK, et al. Toward a definition of a threshold for harmless doses to the anal-sphincter region and the rectum. Int $\mathrm{J}$ Radiat Oncol Biol Phys 2005;61:1035-1044.

[39] Kienle, P, Abend, F, Dueck, M, Abel, U, Treiber, M, Riedl, S. Influence of intraoperative and postoperative radiotherapy on functional outcome in patients undergoing standard and deep anterior resection for rectal cancer. Dis Colon Rectum 2006;49:557-567.

[40] Bujko, K, Rutkowski, A, Chang, GJ, Michalski, W, Chmielik, E, Kusnierz, J. Is the 1-cm Rule of Distal Bowel Resection Margin in Rectal Cancer Based on Clinical Evidence? A Systematic Review. Ann Surg Oncol 2011. DOI 10.1245/s10434-011-2035-2. 
[41] Park, IJ, Kim, JC. Adequate length of the distal resection margin in rectal cancer: from the oncological point of view. J Gastrointest Surg;14:1331-1337.

[42] Nijkamp, J, de Jong, R, Sonke, JJ, van Vliet, C, Marijnen, C. Target volume shape variation during irradiation of rectal cancer patients in supine position: comparison with prone position. Radiother Oncol 2009;93:285-292.

[43] Valentini, V, Aristei, C, Glimelius, B, et al. Multidisciplinary Rectal Cancer Management: 2nd European Rectal Cancer Consensus Conference (EURECA-CC2). Radiother Oncol 2009;92:148163.

[44] Fujishiro, M, Yahagi, N, Kakushima, N, et al. Outcomes of endoscopic submucosal dissection for colorectal epithelial neoplasms in 200 consecutive cases. Clin Gastroenterol Hepatol 2007;5:678-683.

[45] Kam, MH, Wong, DC, Siu, S, Stevenson, AR, Lai, J, Phillips, GE. Comparison of magnetic resonance imaging-fluorodeoxy- glucose positron emission tomography fusion with pathological staging in rectal cancer. Br J Surg 2010;97:266-268.

[46] Llamas-Elvira, JM, Rodriguez-Fernandez, A, Gutierrez-Sainz, J, et al. Fluorine-18 fluorodeoxyglucose PET in the preoperative staging of colorectal cancer. Eur J Nucl Med Mol Imaging 2007;34:859-867.

[47] Lambregts, DM, Beets, GL, Maas, M, et al. Accuracy of Gadofosveset-enhanced MRI for Nodal Staging and Restaging in Rectal Cancer. Ann Surg 2011;253:539-545.

[48] Wanet, M, Lee, JA, Weynand, B, et al. Gradient-based delineation of the primary GTV on FDGPET in non-small cell lung cancer: a comparison with threshold-based approaches, CT and surgical specimens. Radiother Oncol 2011;98:117-125.

[49] Aerts, HJ, van Baardwijk, AA, Petit, SF, et al. Identification of residual metabolic-active areas within individual NSCLC tumors using a pre-radiotherapy (18)Fluorodeoxyglucose-PET-CT scan. Radiother Oncol 2009;91:386-392.

[50] Lambin, P, Petit, SF, Aerts, HJ, et al. The ESTRO Breur Lecture 2009. From population to voxel-based radiotherapy: exploiting intra-tumor and intra-organ heterogeneity for advanced treatment of non-small cell lung cancer. Radiother Oncol 2010;96:145-152.

[51] van den Bogaard, J, Janssen, MH, Janssens, G, et al. Residual metabolic tumor activity after chemo-radiotherapy is mainly located in initially high FDG uptake areas in rectal cancer. Radiother Oncol 2011;99(2):137-41. 
Part II

Response prediction in rectal cancer

treatment

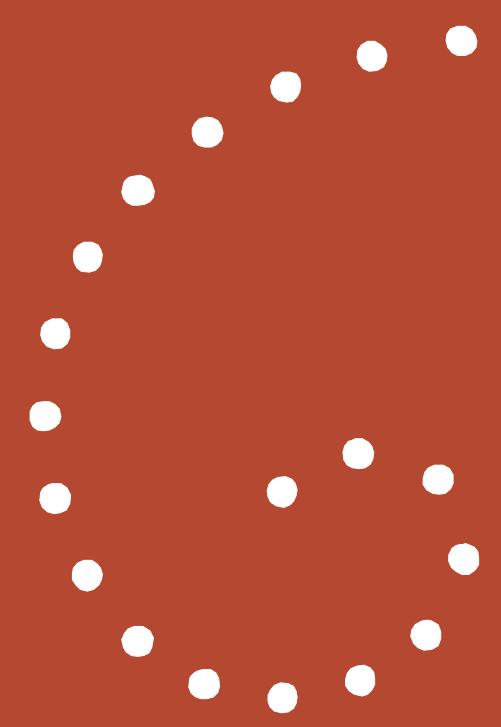





\section{Chapter 4 \\ Development and external validation of a predictive model for pathological complete response of rectal cancer patients including sequential PET-CT imaging}

Ruud G. van Stiphout, Guido Lammering", Jeroen Buijsen*, Marco H. Janssen, Maria Antonietta Gambacorta, Pieter Slagmolen, Maarten Lambrecht, Domenico Rubello, Marcello Gava, Alessandro Giordano, Eric O. Postma, Karin Haustermans, Carlo Capirci, Vincenzo Valentini, Philippe Lambin

* Equal contribution

Radiotherapy and Oncology, 


\section{Purpose}

To develop and validate an accurate predictive model and a nomogram for pathologic complete response ( $\mathrm{PCR}$ ) after chemoradiotherapy (CRT) for rectal cancer based on clinical and sequential PET-CT data. Accurate prediction could enable more individualised surgical approaches, including less extensive resection or even a wait-andsee policy.

\section{Methods and materials}

Population based databases from 953 patients were collected in four different institutes and divided into three groups: clinical factors (training: 677 patients, validation: 85 patients), pre-CRT PET-CT (training: 114 patients, validation: 37 patients) and post-CRT PET-CT (training: 107 patients, validation: 55 patients). A PCR was defined as ypTONO reported by pathology after surgery. The data were analysed using a linear multivariate classification model (support vector machine), and the model's performance was evaluated using the area under the curve (AUC) of the receiver operating characteristic (ROC) curve.

\section{Results}

The occurrence rate of $\mathrm{pCR}$ in the datasets was between $15 \%$ and $31 \%$. The model based on clinical variables ( $A \cup C_{\text {train }}=0.61 \pm 0.03, A \cup C_{\text {validation }}=0.69 \pm 0.08$ ) resulted in the following predictors: $\mathrm{CT}$ - and $\mathrm{CN}$-stage, and tumor length. Addition of pre-CRT PET data did not result in a significantly higher performance $\left(A \cup C_{\text {train }}=0.68 \pm 0.08, A \cup C_{\text {validation }}=0.68 \pm 0.10\right.$ ) and revealed maximal radioactive isotope uptake $\left(S U V_{\max }\right)$ and tumor location as extra predictors. The best model achieved was based on the addition of post-CRT PET-data ( $A \cup C_{\text {train }}=0.83 \pm 0.05, A \cup C_{\text {validation }}=0.86 \pm 0.05$ ) and included the following predictors: tumor length, post-CRT SUV $\max$ and relative change of SUV $V_{\text {max }}$. This model performed significantly better than the clinical model $\left(p_{\text {train }}<0.001, p_{\text {validation }}=0.056\right)$.

\section{Conclusions}

The model and the nomogram developed based on clinical and sequential PET-CT data can accurately predict $\mathrm{PCR}$, and can be used as a decision support tool for surgery after prospective validation. 


\section{Introduction}

Over the past decades, treatment outcomes for rectal cancer have changed dramatically. A better surgical technique, total mesorectal excision (TME), and the introduction of neoadjuvant treatments in locally advanced rectal cancer (LARC) have significantly decreased the risk of locoregional relapse [1, 2]. In the last nine years at least seven published phase III trials have evaluated the role of adjuvant radiotherapy in rectal cancer [3]. These have provided an evidence base demonstrating the efficacy of both preoperative radiotherapy and preoperative concurrent chemotherapy (CRT). CRT has been reported to induce significant tumor downsizing and downstaging [4-6], with a pathologic complete response (pCR) after CRT observed in $10 \%-30 \%$ of patients $[2,4-8]$. Although some studies showed no correlation [9], many others reported that patients showing a PCR following preoperative CRT have improved long-term outcomes including excellent local control rates and disease-free survival, regardless of their initial clinical T- and N-stages [10-13].

However, despite the often phenomenal downsizing and sometimes even complete pathological responses after CRT, these patients are still operated with a standard extended surgical procedure due to the lack of reliable accurate preoperative diagnostic tools. However, it may be questioned whether a standard resection is still necessary, considering the good outcome of these patients reported with less invasive treatments [14, 15]. If accurately selected, patients with a complete response (no residual tumor) may undergo a less extensive resection or even a so called 'wait-and-see' policy. Compared to standard surgery, the benefits of these treatments are reduced morbidity and mortality (e.g., anastomotic leakage, relaparotomy, wound and pelvic infection, abscess, colostomy, chronic wound healing disturbances, faecal or urinary incontinence and sexual dysfunction), improved quality of life and reduced treatment costs.

Thus, an accurate prediction of pCR can help in the selection of patients for more optimised treatment, sphincter-preserving surgery, less extensive resection, more intense radiation treatment, or even delayed surgery with a 
wait-and-see policy $[2,3,16]$. These considerations led to the overall goal of this study: to develop an accurate, data-driven model to predict pathologic complete response for rectal cancer patients as decision support for more individualised treatment approaches in the future.

The clinical variables associated with a better response to preoperative CRT include circumferential tumor extent, tumor differentiation, preoperative classification, carcinoembryonic antigen (CEA) level, distance from anal verge, and time to surgery $[6,17,18]$. Recently, it has also been suggested that PET imaging might be correlated with tumor response after CRT in locally advanced rectal cancer. However, the studies involved used only a small number of patients, which meant that contradictory results were found. Further, only semiquantitative PET measurements were used and analysed with univariate statistics [4, 5, 7, 19-26]. Multivariate analysis was performed in only one study, whose results lacked statistical significance [27]. Notably, no studies verified and validated their results with external datasets, despite the fact that this represents an important prerequisite for the generalizability of prediction models for other institutes.

In the current study, population based data from four different institutes were collected and used to train and validate predictive models for PCR. We hypothesised that the addition of PET imaging data to clinical variables significantly increases the performance of prediction models for pCR after CRT as compared to models based on clinical data alone.

The study was performed within the framework of a decision support system based on centralised datasets. The increasing amount of available patient information requires automatic methods for model building and analysis. Machine learning methods can be used to update the models continuously by feeding them with information of new patients. The increasing complexity of prediction models, too, means that the representation and interpretation of the results also become more important. Tools to enhance interpretation for the 
clinic include visualisation techniques such as nomograms and graphical networks. Nomograms are statistical tools that enable users to calculate the overall probability of a specific clinical outcome for an individual patient [28]. In this study, the nomogram with the highest accuracy for the prediction of $\mathrm{pCR}$ is provided.

\section{Methods and materials}

\section{Study population}

Six population based datasets were collected in four institutes: Maastro Clinic (GROW, MUMC, Maastricht, the Netherlands), Università Cattolica del S.Cuore (Rome, Italy), S. Maria della Misericordia Hospital (Rovigo, Italy) and University Hospital Gasthuisberg (Leuven, Belgium). In total, 953 patients met the criteria for inclusion: long-course RT with neoadjuvant chemotherapy and the availability of pathological outcome for PCR. Of these, 276 patients underwent a pre-CRT PET scan (one week before the start of CRT), and 169 patients had both pre- and post-CRT PET scans (one week before surgery, and six to eight weeks after the end of CRT). The sequential PET data from Rovigo have already been published as a prospective study[20], the Leuven data were collected prospectively for the BioCare project (LSHC-CT-2204-505785) and the rest of the data were gathered for a population-based study registered in the Dutch Trial Register (NTR2166). All compositions of the cohorts were approved by the local IRB committees. The patient characteristics are reported in table 1 . The datasets were divided into three groups, based on PET data availability: 1. clinical variables only, 2. clinical variables with pre-CRT PET variables (PET-pre), 3. clinical variables with both pre- and post-CRT PET variables (PET-post). For each group, a training set and an external validation set were defined. The training sets were used to identify the $\mathrm{PCR}$ predictors, while the validation sets were used to test the performance of the models in other centres. Datasets from a single centre with the highest number of patients were used for training. A dataset was deemed not useful for external validation if it originated from the same centre as the corresponding training set. The definition of the different 
combined training and validation sets is explained in table 2, based on the datasets in table 1.

The available clinical variables were age, gender (0: female, 1: male), clinical tumor (cT) and nodal (cN) stage, and two variables based on MRI (or endoscopy if MRI was unavailable): tumor location categorised in three levels (1: low, 0-5 $\mathrm{cm}$ from anal verge; 2 : $\mathrm{mid}, 5-10 \mathrm{~cm}$ from anal verge; 3: high, $>10 \mathrm{~cm}$ from anal verge) and tumor length $(\mathrm{cm})$. For the patients who had PET-CT scans, the tumors were semi-automatically contoured at Maastro Clinic using dedicated software (TrueD, Siemens Medical, Erlangen, Germany). Standardised uptakevalue (SUV) thresholding was based on the tumor-to-background signal ratio, with the gluteus muscle as reference background [29, 30]. From the resulting tumor contour, maximal tumor diameter (MaxD), gross tumor volume (GTV), and maximal and mean SUV values within the GTV were calculated. If the postCRT PET-CT scan was available, the same variables were scored, and a response index (RI) for each variable was calculated. For variable $X$, the response index is the relative percent difference between the value of the post-CRT and pre-CRT and it was defined as $\mathrm{RI}=\left(\mathrm{X}_{\text {pre }}-\mathrm{X}_{\text {post }}\right) / \mathrm{X}_{\text {pre }}{ }^{\star} 100 \%$. Thus, six variables were evaluated for the clinical dataset, 10 for the PET-pre dataset and 18 for the PETpost dataset. From these sets, the models selected subgroups of variables with significant predictive value for $\mathrm{PCR}$.

All patients underwent surgery. Pathological complete response was defined as ypTON0, extracted from the pathologic reports of surgical specimens. All other cases (ypT+ and/or ypN+) were considered non-responders, making the $\mathrm{pCR}$ a binary outcome $(0 / 1)$. The specimens were not re-evaluated centrally but the pathology protocols were very similar between institutes $(3-5 \mathrm{~mm}$ slices of rectum tumor, intensified evaluation on several blocks of tissue at the tumor site, evaluation on 2-3 sublevels when no tumor tissue was found in initial block).

\section{Statistical analysis}

Missing values in the dataset were substituted by the mean [31]. This method 
performed similarly to other, more complex substitution methods for small percentages of missing values (e.g., expectation-maximisation imputation, regression estimation). No variables in the datasets exceeded $5 \%$ of missing values. Patients who missed tumor location and length in the clinical datasets (Roma: $n=132$ and Maastricht: $n=29$ ) were excluded because of too large amounts of missing data for these variables. All patient numbers stated in this paper were extracted after the missing value procedure. To compare the weights of significance assigned to the variables by the model, all variables were normalised by subtracting the mean, and then divided by the standard deviation.

To classify the complete responders and non-responders, a linear multivariate method suitable for binary classification from the machine learning field was used: the support vector machine (SVM) [32].The SVM variant used (proximal SVM or pSVM) performs equally accurately but much faster than normal support vector machines [33]. The different datasets' performances in predicting PCR were evaluated by analysing the area under the curve (AUC) of the receiver operating characteristic (ROC) curve[34]. The maximum value of the AUC is 1.0, indicating a perfect prediction model; a value of 0.5 indicates a random chance of correct prediction.

To select the variables that contribute to PCR prediction, an exhaustive feature search was performed, with all possible variable combinations used as input for the pSVM model. The set of variables resulting in the highest AUC was selected as the final predictive set. To avoid over-fitting of the model through selection of the highest AUC, the variable sets resulting in AUCs that deviated less than $5 \%$ from the maximal AUC were compared to the final variable set. If conflicts occurred or if variables did not contribute significantly, selected variables were interchanged by considering their prevalence in the highly predictive sets, the factor analysis and the Spearman correlation coefficient (i.e., highly correlated and dependent variables are not present in the same predictive set). Furthermore, an extra univariate analysis was performed using the Wilcoxon rank sum test. 
Classification methods normally require at least several hundred cases. Because of the relatively small number of available patients, two extra evaluation methods were used. The first was leave-one-out (LOO) cross-validation, used to calculate an AUC for the training set. In LOO cross-validation, a single patient is selected from the original training dataset and used as the validation dataset, while the data from the remaining patients are used to train the model. This is repeated until all patients have been selected once for validation. However, no LOO cross-validation was used for the external dataset. The second evaluation method was bootstrapping, which results in a more accurate approximation of the real dataset distribution [35]. This means that 1000 datasets are generated from the original dataset containing $n$ patients by selecting these $n$ patients, but with resampling (i.e., patients can be present in the dataset more than once). For every bootstrapped dataset, an AUC was calculated. The mean AUC with the corresponding standard deviation was then calculated with size 1000 . This nonparametric method allows comparison of the confidence intervals of the AUCS of different datasets without making assumptions about the AUC distributions [36]. The distribution of the difference in mean AUC ( $\triangle A \cup C$ ) between the datasets was tested by calculating the two-sided p-value, i.e., the fraction of $\triangle A U C$ samples smaller or larger than zero (depending on the dominant sign of $\triangle \mathrm{AUC})$.

Nomograms can reduce statistical predictive models to a single numerical estimate of the probability of an event, and visualise the effect of each selected variable on this probability [37]. The model output of the PSVM models consists of assigned weights for each variable and an offset. The probability of a patient having a pCR can be calculated using logistic regression on the pSVM output [38]. The complete procedure to convert SVM output to a nomogram is described in detail elsewhere [39]. Developing a nomogram requires threshold selection in the ROC curve. For response prediction specificity is most important, because it is not preferred to predict non-responders as responders, which would result in under-treatment. Therefore, the threshold was selected in such a way that at least $90 \%$ of non-responders were correctly predicted. Partial 
ROC curve optimisation has been tested but it had no gain for specificity compared to overall AUC maximisation [40]. Calibration of the nomogram, i.e., the agreement between predicted probability of complete response and true probability in the population, was performed by an assessment of the overall agreement and the Hosmer-Lemeshow statistic in four subgroups of patients in the validation data. The nomogram algorithm was implemented in MATLAB (version 7.1, MathWorks Inc., Natick, MA), as were all algorithms described in this section. 


\section{Results}

The occurrence of pCR in the patient population varied between $15 \%$ and $31 \%$ (mean: $21.8 \%$, SD: $5.4 \%$ ) depending on the dataset (Table 1). A first evaluation of CRT's effect on the tumor demonstrated significant downsizing of the tumor in the PET-CT, and a significant decrease in metabolic activity within the tumor (Figure 1). Both gross tumor volume and maximal SUV decreased significantly between the pre- and post-CRT PET-CT scans $(p<0.001)$.

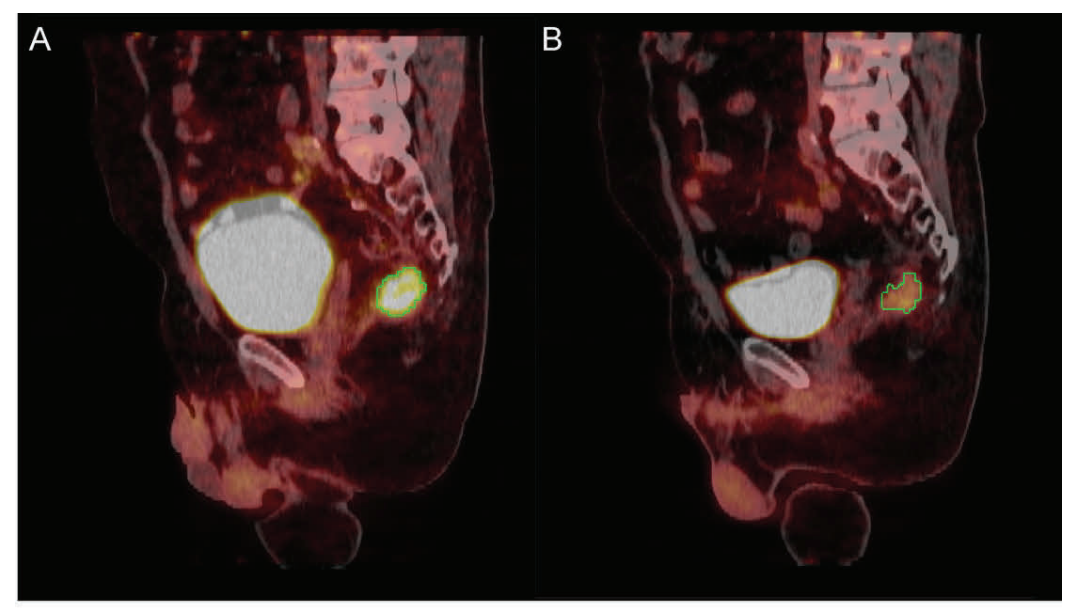

C

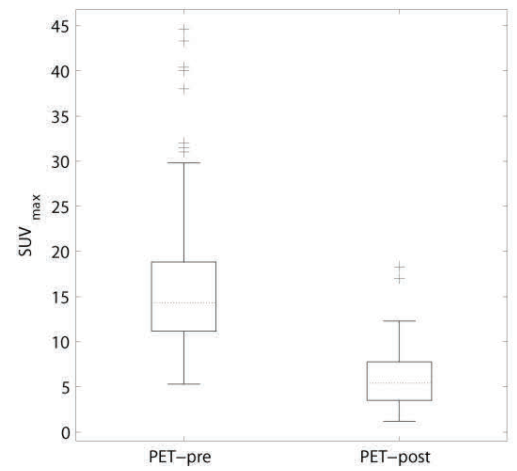

D

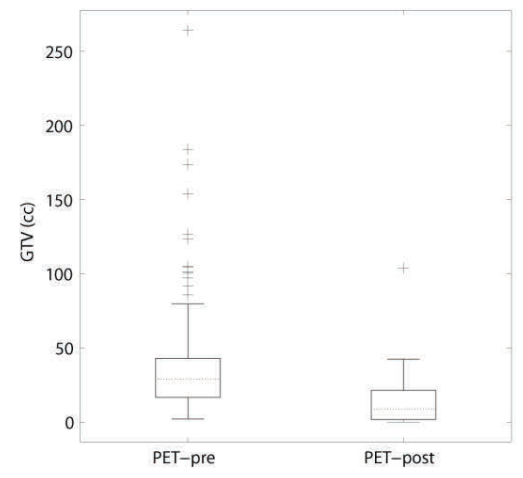

Figure 1 (A) Tumor contour in a fused FDG-PET-CT made pre-CRT. (B) Corresponding post-CRT FDGPET-CT scan with tumor contour. (C) Boxplot of SUV $\max$ on PET-scans made pre-CRT and post-CRT; significant decrease: $p<0.001$ (D) Boxplot of the GTV for the case of pre-CRT and post-CRT; significant decrease: $p<0.001$. 
Table 1 Patient characteristics for six datasets from four different institutes. Clinical, PET-pre and PET-post groups are defined. Percentages of the total patient numbers are given for binary or ordinal variables. Mean and standard deviation (SD) are given for continuous variables. $x$ denotes missing values. $\mathrm{RT}=$ Radiotherapy, $\mathrm{PF}=$ per fraction .

\begin{tabular}{|c|c|c|c|c|c|c|}
\hline Center & Maastricht & & Rome & & Rovigo & Leuven \\
\hline Dataset & M1 & M2 & $\mathrm{R} 1$ & $\mathrm{R} 2$ & $\mathrm{C} 1$ & L1 \\
\hline Period & 2004-2006 & 2004-2006 & $1984-2008$ & $2007-2008$ & $2003-2007$ & 2005-2007 \\
\hline \# Patients & 114 & 21 & 677 & 18 & 107 & 16 \\
\hline Clinical & Validation & - & Training & - & - & - \\
\hline PET-pre & Training & - & - & Validation & - & Validation \\
\hline PET-post & - & Validation & - & Validation & Training & Validation \\
\hline \multicolumn{7}{|l|}{ Gender (\%) } \\
\hline Male & 63 & 67 & 63 & 83 & 74 & 81 \\
\hline Female & 37 & 33 & 37 & 17 & 26 & 19 \\
\hline \multicolumn{7}{|l|}{ Age } \\
\hline Mean & 65.6 & 66.1 & 61.3 & 60.4 & 66.3 & 58.6 \\
\hline SD & 10.0 & 10.6 & 10.2 & 7.1 & 10.8 & 10.1 \\
\hline \multicolumn{7}{|l|}{ cT (\%) } \\
\hline 1 & 0 & 0 & 0 & 0 & 0 & 0 \\
\hline 2 & 1 & 0 & 3 & 11 & 0 & 0 \\
\hline 3 & 68 & 81 & 86 & 56 & 90 & 94 \\
\hline 4 & 30 & 14 & 11 & 33 & 10 & 6 \\
\hline$x$ & 1 & 5 & 0 & 0 & 0 & 0 \\
\hline \multicolumn{7}{|l|}{ cN (\%) } \\
\hline 0 & 25 & 38 & 23 & 17 & 51 & 0 \\
\hline 1 & 48 & 48 & 45 & 33 & 38 & 62 \\
\hline 2 & 26 & 10 & 30 & 50 & 10 & 38 \\
\hline$x$ & 1 & 4 & 2 & 0 & 1 & 0 \\
\hline \multicolumn{7}{|l|}{ cM (\%) } \\
\hline 0 & 73 & 71 & 100 & 94 & 100 & 100 \\
\hline 1 & 25 & 19 & 0 & 6 & 0 & 0 \\
\hline$x$ & 2 & 10 & 0 & 0 & 0 & 0 \\
\hline \multicolumn{7}{|l|}{ ypTONO (\%) } \\
\hline No & 85 & 81 & 80 & 78 & 76 & 69 \\
\hline Yes & 15 & 19 & 20 & 22 & 24 & 31 \\
\hline \multicolumn{7}{|l|}{ RT dose } \\
\hline Mean & 50.4 & 50.4 & 49.0 & 52.7 & 55.7 & 45.7 \\
\hline SD & 0 & 0 & 5.5 & 3.3 & 3.1 & 1.8 \\
\hline RT dose PF & 1.8 & 1.8 & 1.8 & 1.8 & 2.2 & 1.8 \\
\hline \# Chemo Types & 1 & 1 & 11 & 2 & 1 & 1 \\
\hline
\end{tabular}


Table 2 Predictor selection and ROC analysis. Predictive variables are given with their corresponding assigned normalized weights from multivariate analysis (MVA). For each variable the $\mathrm{p}$-value from univariate analysis (UVA) is given. Mean AUC and standard deviation (SD) are given for each variable set. $\mathrm{RI}=$ response index, SUV = standard uptake value, $\mathrm{MaxD}=$ maximal diameter (PETCT).

\begin{tabular}{|c|c|c|c|c|c|c|c|}
\hline Variable set & Type & Size & Predictors (MVA) & $\begin{array}{l}\text { Weights } \\
\text { (MVA) }\end{array}$ & $\begin{array}{l}\text { p-value } \\
\text { (UVA) }\end{array}$ & AUC & SD \\
\hline \multirow[t]{4}{*}{ Clinical } & Training & 677 & Tumor length & -0.085 & $<0.001$ & 0.61 & 0.03 \\
\hline & (R1) & & cT-stage & -0.074 & 0.001 & & \\
\hline & & & cN-stage & -0.060 & 0.001 & & \\
\hline & $\begin{array}{l}\text { Validation } \\
\text { (M1) }\end{array}$ & 85 & - & - & - & 0.69 & 0.08 \\
\hline \multirow[t]{5}{*}{ Clinical + PET-pre } & Training & 114 & MaxD pre & -0.12 & 0.003 & 0.68 & 0.08 \\
\hline & (M1) & & cN-stage & -0.12 & 0.001 & & \\
\hline & & & Tumor location & 0.094 & 0.84 & & \\
\hline & & & SUV $V_{\text {max-pre }}$ & -0.087 & 0.29 & & \\
\hline & $\begin{array}{l}\text { Validation } \\
\text { (R2, L1) }\end{array}$ & 34 & - & - & - & 0.68 & 0.10 \\
\hline Clinical + PET-pre + & Training & 107 & $\mathrm{Rl}_{\text {suVmax }}$ & 0.20 & $<0.001$ & 0.83 & 0.05 \\
\hline \multirow[t]{3}{*}{ PET-post } & (C) & & Tumor length & -0.20 & $<0.001$ & & \\
\hline & & & SUV $V_{\text {max-post }}$ & -0.14 & $<0.001$ & & \\
\hline & $\begin{array}{l}\text { Validation } \\
\text { (M2, R2, L1) }\end{array}$ & 55 & - & - & - & 0.86 & 0.05 \\
\hline
\end{tabular}

Table 2 shows the predictor selection results and the ROC curve analysis. For the clinical dataset, the univariate analysis reveals three variables significantly associated with pCR (95\% confidence interval): tumor length $(\mathrm{p}<0.001), \mathrm{cN}$ stage $(p=0.001)$, and $c T$-stage $(p=0.001)$. These variables were also selected in the multivariate analysis. The normalised weights assigned to them by the pSVM model are tumor length (-0.085), cT-stage (-0.074), and cN-stage $(-0.060)$. The selected variables were ranked in importance (i.e., weights). The sign of the weights can be interpreted by the effect on the probability of a pCR. For a negative sign, this probability decreases when the variable increases. For the clinical dataset, this means that the probability of a PCR increases for small tumor lengths and low cT- and $\mathrm{cN}$-stages. The predictive performance of the clinical dataset for $\mathrm{PCR}$, expressed by the AUC of the ROC curve, is $0.61 \pm 0.03$ (mean \pm SD) for the training set and $0.69 \pm 0.08$ for the external validation set. 
For the dataset with pre-CRT PET data, the multivariate analysis selected these variables (ranked by weight): maximal diameter (-0.12), cN-stage (-0.12), tumor location (0.094), and SUV $\max (-0.087)$. This resulted in a high probability of pCR for patients with small maximal tumor diameters, low cN-stage, high tumor locations, and small maximal metabolic activity. Maximal diameter $(p=0.003)$ and $c N$-stage $(p=0.001)$ were selected by univariate analysis, while the other two variables were not. The AUCs for the training and validation set were both 0.68 , but the SD differed ( 0.08 and 0.10 respectively).

The dataset including the post-CRT PET data resulted in the highest performance: $\mathrm{AUC}_{\text {train }}=0.83 \pm 0.05$ and $\mathrm{AUC}_{\text {validation }}=0.86 \pm 0.05$. The response

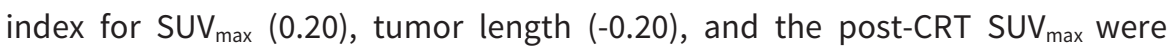
found to be predictive for $\mathrm{PCR}$ and significantly associated with $\mathrm{PCR}$ in the univariate analysis $(p<0.001)$.

In evaluating the predictive value of the additional PET data to the clinical data, only the AUCs of the post-CRT PET data differed significantly from the clinical dataset AUC (Figure 2). The p-value for the AUC difference for the training set was $<0.001$, while that for the validation sets was 0.056 (just outside the $95 \%$ confidence interval). When only post-CRT PET data were used for the models (i.e., no clinical variables), the significant difference between the AUCs and the clinical dataset was no longer observed (training: $p=0.47$, validation: $p=0.58$ ). This indicated that a combination of both clinical and PET data was required to reach a significantly higher performance when using PET as a predictive imaging modality.

The assigned weights for all the predictors formed the basis for the construction of the nomogram. The nomogram based on the post-CRT dataset is provided in Figure 3. The nomogram performs with a sensitivity of 0.62 and a specificity of 0.88 for the validation data. In the training phase these were respectively 0.65 and 0.90 . The calibration of the nomogram (Figure 4) with the validation data reveals that the overall predicted and the actual probability are equal ( $23.6 \%$, 
$\mathrm{OR}=1.0$ ). If the validation data are divided into four equally numbered groups, the Hosmer-Lemeshow test results in a p-value of 0.78 , which means a good calibration in this test $(p>0.05)$. The linear fit through these probabilities results in a slope of 1.02 with $\mathrm{R}^{2}$ of 0.99 , confirming a good balance between calibration and discrimination.
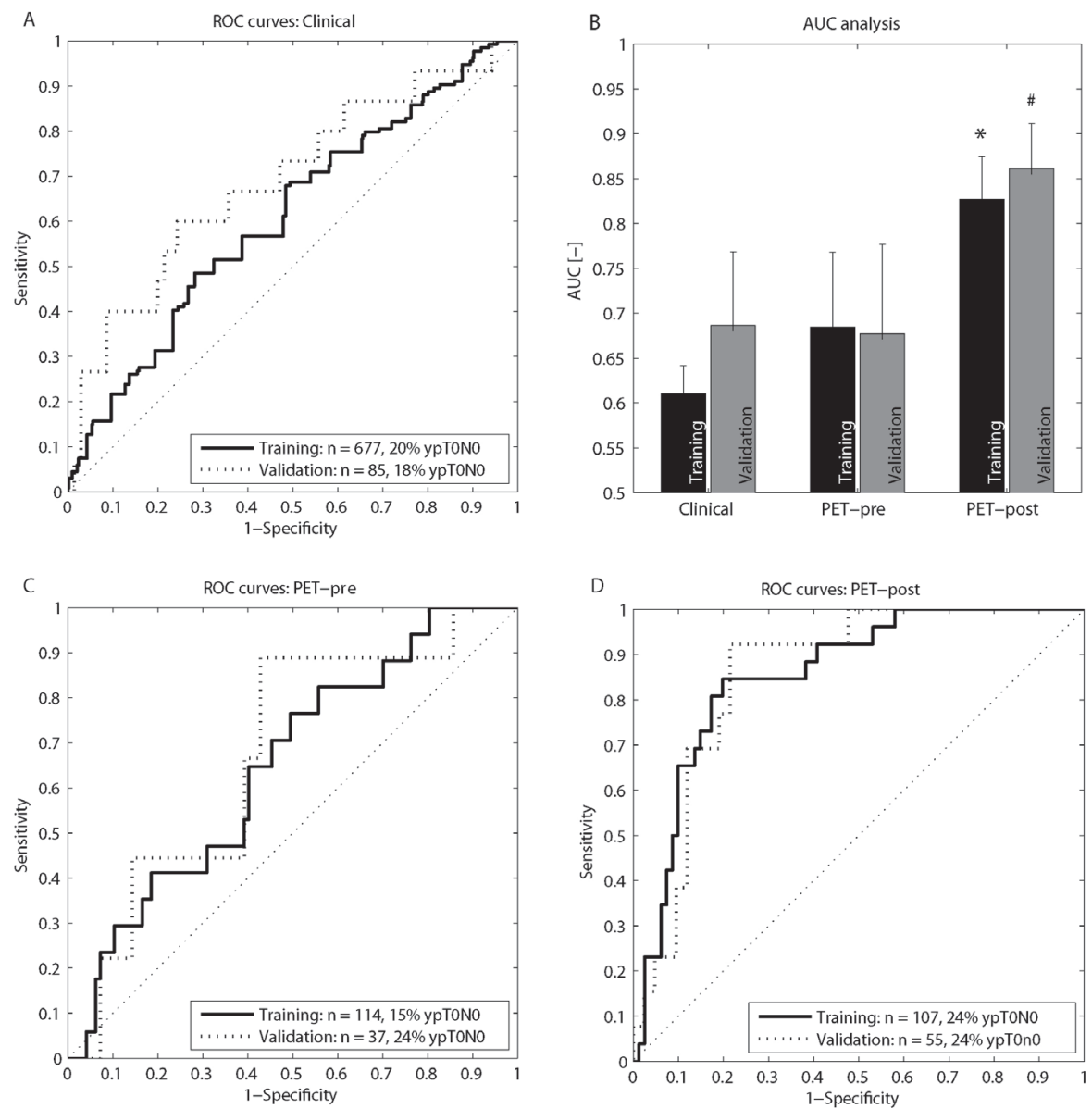

Figure 2 ROC curves of training and validation datasets for the clinical set (A), the PET pre-CRT set (C) and the PET post-CRT set (D). The straight dashed line represents a random prediction model. The bar plot (B) shows the corresponding mean AUC for each dataset and the standard deviation (error bars). There was a significant difference with clinical datasets of $\left(^{*}\right) p<0.05$ and $(\#) p<0.06$. 

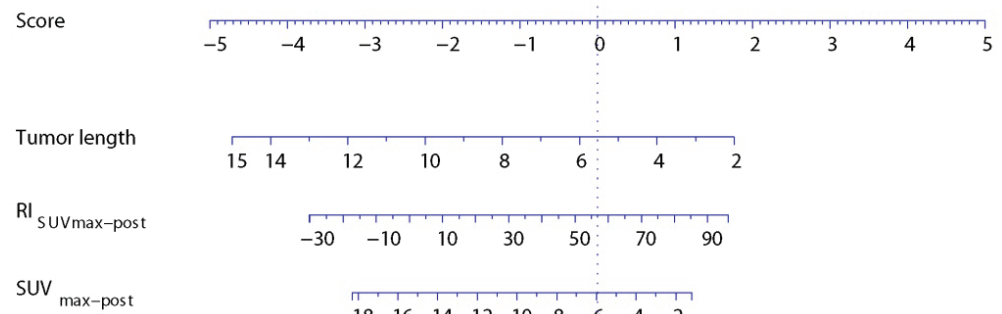

$$
\text { max-post }
$$
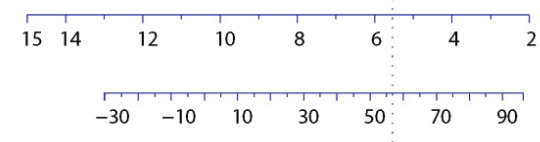

$\begin{array}{llllllllll}18 & 16 & 14 & 12 & 10 & 8 & 6 & 4 & 2\end{array}$

Sum of scores

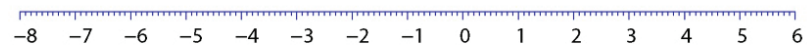

Probability of $\mathrm{pCR}$

$\begin{array}{lllllllllll}0.05 & 0.1 & 0.2 & 0.3 & 0.5 & 0.7 & 0.8 & 0.9 & 0.95\end{array}$

Figure 3 Nomogram for PET post-CRT dataset. A score for each predictor can be read out at the top scale (Score). All summed scores (Sum of scores scale) can be converted directly to the probability of responding with a PCR (ypTONO). The probability scale is the only logarithmic scale.

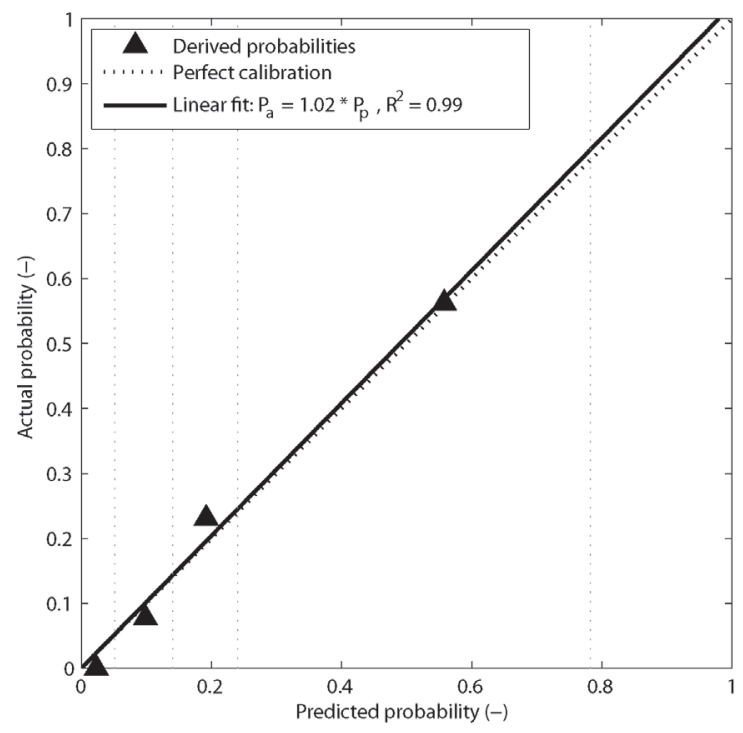

Figure 4 Calibration of the nomogram for the validation data. For the four equally numbered subgroups (vertical lined intervals in figure), the predicted probability of a PCR and the actual fraction in the population were evaluated. The dashed line represents perfect calibration and the solid line is the linear fit of the calibration data. 


\section{Discussion}

We have developed predictive models based on clinical and PET-based data for pathologic complete response in patients diagnosed with rectal cancer. The performance of these models was externally validated using patient cohorts from different institutes treated with long-course preoperative chemoradiotherapy. The models showed that the accuracy of the predictions increased over time, i.e., when more information became available. Information from PET-CT scans significantly improved the performance of the models.

The significant difference in AUCs that we reported between the performance of the clinical model and the post-CRT PET data model reflects what others have found in their post-treatment PET analyses; like us, some have reported (significant) indications that the response index and post-treatment SUV $\mathrm{max}_{\max }$ are predictive for response, while the pre-treatment PET data do not provide enough predictive power $[12,19,27]$. However, our PET-based models also contain clinical variables, which appeared to be necessary to obtain the high performance provided in Table 2. The most important clinical variables were tumor length and maximal diameter, which were selected in the models and are significantly correlated (spearman $\rho=0.55, p<0.001$ ). Overall, this means that the dominant tumor dimension in combination with (differences in) the maximal metabolic activity inside the tumor is the most predictive variable set for PCR, which was confirmed in the external datasets.

Whether the corresponding AUC of 0.86 is accurate enough for clinical practice depends on the choice of the threshold in the ROC curve. A high specificity is preferred over a high sensitivity to avoid possible under-treatment (less surgery when surgery is required) rather than over-treatment (standard treatment when less surgery could have been considered). The provided nomogram focuses on specificity (training: 0.90 , validation 0.88 ). Selecting higher specificities results in fast decreasing sensitivities. Careful follow-up is therefore necessary for the patients selected for a 'wait-and-see' policy to detect any possible local 
recurrences early on. To gain more specificity in the future, the addition of new variables and the other classification methods would have to be considered.

The nomogram performs well, i.e., the distribution of the probability of a pCR provided by the nomogram represents the true distribution in the data, confirmed by overall calibration, calibration of the slope and HosmerLemeshow test (figure 4). Because of the number of events and the division of the patient cohorts into few probability intervals, the higher probabilities occur much less frequently and are thus the least accurate. Therefore, prospective validation of the model and the nomogram is required to ensure sufficient statistical power for clinical application of the models. Besides the number of patients to increase the models' accuracy, more predictors could be added to increase the models' performance, including biological variables such as gene signatures [41] and blood biomarkers, and also more imaging variables from (perfusion) CT and (diffusion) MRI. The first indications have also appeared that PET-CT data during CRT may be highly predictive for response [25, 26, 42]. This time point is more favourable than post-CRT because of the possibility of earlier treatment changes and the decreased presence of inflammatory rectum cases, potentially causing impaired evaluation of fused PET-CT scans. After prospective validation of the model, an intervention trial with less surgery for patients with a high probability for pCR will be performed.

The population based collected datasets date back five years, except for the clinical Roma database, which was collected from 1984 onward. Therefore, this dataset shows a higher variety in treatment schemes than the other datasets. This could explain the discrepancy of the higher prediction performance of the clinical validation set. On the other hand, the validation set is much smaller, implying that the distribution of data could not be representative of the true distribution. The consequence of population based data collection is that treatment protocols are not well tuned. This results in, for example, small differences in irradiation schemes and deviations in the evaluation of pathology outcome. Ideally, pathology is reviewed centrally to reduce the intra- and inter- 
observer variability for the outcome measure. However, in this study the quality of pathology is acceptable because of the prospective nature of most datasets and because the outcome was limited to only complete response evaluation. Also, glucose correction for SUV values was not applied to all datasets. However, minor variation in treatment schemes can be seen as an advantage because it leads to higher generalizability for other centres. In other words, the model still performs well, despite the disparities mentioned here.

In conclusion, we have shown that sequential PET-CT data in combination with clinical variables significantly increase the performance of prediction models for pathologic complete response. So far, this is the largest study of its kind and the only one that used external datasets for validation. The dominant tumor dimension and the maximal uptake of radioactive isotopes in the tumor as well as its relative difference between PET scans were found to be the best predictors for $\mathrm{pCR}$ resulting in very good overall performance AUC's of 0.83 and 0.86 for training and validation, respectively. Including also biological and other imaging variables will probably further improve the performance. When prospectively validated, the model and the nomogram therefore provide a valuable decision support for more individualised treatment approaches in the future.

Note: The predictive models in this paper are published on the website http://www.predictcancer.org. 


\section{References}

[1] Adjuvant radiotherapy for rectal cancer: a systematic overview of 8,507 patients from 22 randomised trials. Lancet. 2001;358:1291-304.

[2] Valentini V, Beets-Tan R, Borras JM, Krivokapic Z, Leer JW, Pahlman L, et al. Evidence and research in rectal cancer. Radiother Oncol. 2008;87:449-74.

[3] Valentini V, Aristei C, Glimelius B, Minsky BD, Beets-Tan R, Borras JM, et al. Multidisciplinary Rectal Cancer Management: 2nd European Rectal Cancer Consensus Conference (EURECACC2). Radiother Oncol. 2009;92:148-63.

[4] Capirci C, Rampin L, Erba PA, Galeotti F, Crepaldi G, Banti E, et al. Sequential FDG-PET/CT reliably predicts response of locally advanced rectal cancer to neo-adjuvant chemo-radiation therapy. Eur J Nucl Med Mol Imaging. 2007;34:1583-93.

[5] Capirci C, Rubello D, Chierichetti F, Crepaldi G, Fanti S, Mandoliti G, et al. Long-term prognostic value of $18 \mathrm{~F}-\mathrm{FDG}$ PET in patients with locally advanced rectal cancer previously treated with neoadjuvant radiochemotherapy. AJR Am J Roentgenol. 2006;187:W202-8.

[6] Valentini V, Coco C, Cellini N, Picciocchi A, Fares MC, Rosetto ME, et al. Ten years of preoperative chemoradiation for extraperitoneal T3 rectal cancer: acute toxicity, tumor response, and sphincter preservation in three consecutive studies. Int J Radiat Oncol Biol Phys. 2001;51:371-83.

[7] Vliegen RF, Beets-Tan RG, Vanhauten B, Driessen A, Oellers M, Kessels AG, et al. Can an FDGPET/CT Predict Tumor Clearance of the Mesorectal Fascia after Preoperative Chemoradiation of Locally Advanced Rectal Cancer? Strahlenther Onkol. 2008;184:457-64.

[8] O'Neil BH, Tepper JE. Current options for the management of rectal cancer. Curr Treat Options Oncol. 2007;8:331-8.

[9] Pucciarelli S, Toppan P, Friso ML, Russo V, Pasetto L, Urso E, et al. Complete pathologic response following preoperative chemoradiation therapy for middle to lower rectal cancer is not a prognostic factor for a better outcome. Dis Colon Rectum. 2004;47:1798-807.

[10] Vecchio FM, Valentini V, Minsky BD, Padula GD, Venkatraman ES, Balducci M, et al. The relationship of pathologic tumor regression grade (TRG) and outcomes after preoperative therapy in rectal cancer. Int J Radiat Oncol Biol Phys. 2005;62:752-60.

[11] Habr-Gama A, Perez RO, Nadalin W, Nahas SC, Ribeiro U, Jr., Silva ESAH, Jr., et al. Long-term results of preoperative chemoradiation for distal rectal cancer correlation between final stage and survival. J Gastrointest Surg. 2005;9:90-9; discussion 9-101.

[12] Capirci C, Valentini V, Cionini L, De Paoli A, Rodel C, Glynne-Jones R, et al. Prognostic value of pathologic complete response after neoadjuvant therapy in locally advanced rectal cancer: long-term analysis of $566 \mathrm{ypCR}$ patients. Int J Radiat Oncol Biol Phys. 2008;72:99-107.

[13] Rodel C, Martus P, Papadoupolos T, Fuzesi L, Klimpfinger M, Fietkau R, et al. Prognostic significance of tumor regression after preoperative chemoradiotherapy for rectal cancer. $J$ 
Clin Oncol. 2005;23:8688-96.

[14] Borschitz T, Wachtlin D, Mohler M, Schmidberger H, Junginger T. Neoadjuvant chemoradiation and local excision for T2-3 rectal cancer. Ann Surg Oncol. 2008;15:712-20.

[15] Lezoche G, Baldarelli M, Guerrieri M, Paganini AM, De Sanctis A, Bartolacci S, et al. A prospective randomized study with a 5-year minimum follow-up evaluation of transanal endoscopic microsurgery versus laparoscopic total mesorectal excision after neoadjuvant therapy. Surg Endosc. 2008;22:352-8.

[16] Habr-Gama A, Perez RO, Nadalin W, Sabbaga J, Ribeiro U, Jr., Silva e Sousa AH, Jr., et al. Operative versus nonoperative treatment for stage 0 distal rectal cancer following chemoradiation therapy: long-term results. Ann Surg. 2004;240:711-7; discussion 7-8.

[17] Berger C, de Muret A, Garaud P, Chapet S, Bourlier P, Reynaud-Bougnoux A, et al. Preoperative radiotherapy (RT) for rectal cancer: predictive factors of tumor downstaging and residual tumor cell density (RTCD): prognostic implications. Int J Radiat Oncol Biol Phys. 1997;37:619-27.

[18] Das P, Skibber JM, Rodriguez-Bigas MA, Feig BW, Chang GJ, Wolff RA, et al. Predictors of tumor response and downstaging in patients who receive preoperative chemoradiation for rectal cancer. Cancer. 2007;109:1750-5.

[19] Amthauer H, Denecke T, Rau B, Hildebrandt B, Hunerbein M, Ruf J, et al. Response prediction by FDG-PET after neoadjuvant radiochemotherapy and combined regional hyperthermia of rectal cancer: correlation with endorectal ultrasound and histopathology. Eur J Nucl Med Mol Imaging. 2004;31:811-9.

[20] Capirci C, Rubello D, Pasini F, Galeotti F, Bianchini E, Del Favero G, et al. The Role of DualTime Combined 18-Fluorideoxyglucose Positron Emission Tomography and Computed Tomography in the Staging and Restaging Workup of Locally Advanced Rectal Cancer, Treated with Preoperative Chemoradiation Therapy and Radical Surgery. Int J Radiat Oncol Biol Phys. 2009.

[21] Denecke T, Rau B, Hoffmann KT, Hildebrandt B, Ruf J, Gutberlet M, et al. Comparison of CT, MRI and FDG-PET in response prediction of patients with locally advanced rectal cancer after multimodal preoperative therapy: is there a benefit in using functional imaging? Eur Radiol. 2005;15:1658-66.

[22] Guillem JG, Moore HG, Akhurst T, Klimstra DS, Ruo L, Mazumdar M, et al. Sequential preoperative fluorodeoxyglucose-positron emission tomography assessment of response to preoperative chemoradiation: a means for determining longterm outcomes of rectal cancer. J Am Coll Surg. 2004;199:1-7.

[23] Kalff V, Duong C, Drummond EG, Matthews JP, Hicks RJ. Findings on 18F-FDG PET scans after neoadjuvant chemoradiation provides prognostic stratification in patients with locally advanced rectal carcinoma subsequently treated by radical surgery. J Nucl Med. 2006;47:14-22. Melton GB, Lavely WC, Jacene HA, Schulick RD, Choti MA, Wahl RL, et al. Efficacy of 
preoperative combined 18-fluorodeoxyglucose positron emission tomography and computed tomography for assessing primary rectal cancer response to neoadjuvant therapy. J Gastrointest Surg. 2007;11:961-9; discussion 9.

[25] Rosenberg R, Herrmann K, Gertler R, Kunzli B, Essler M, Lordick F, et al. The predictive value of metabolic response to preoperative radiochemotherapy in locally advanced rectal cancer measured by PET/CT. Int J Colorectal Dis. 2009;24:191-200.

[26] Janssen MH, Ollers MC, Riedl RG, Bogaard vdJ, Buijsen J, Stiphout vRG, et al. Accurate prediction of pathological rectal tumor response after 2 weeks of pre-operative radiochemotherapy using FDG-PET-CT imaging. Int J Radiat Oncol Biol Phys. 2009.

[27] Konski A, Li T, Sigurdson E, Cohen SJ, Small W, Jr., Spies S, et al. Use of molecular imaging to predict clinical outcome in patients with rectal cancer after preoperative chemotherapy and radiation. Int J Radiat Oncol Biol Phys. 2009;74:55-9.

[28] Eastham JA, Kattan MW, Scardino PT. Nomograms as predictive models. Semin Urol Oncol. 2002;20:108-15.

[29] Daisne JF, Sibomana M, Bol A, Doumont T, Lonneux M, Gregoire V. Tri-dimensional automatic segmentation of PET volumes based on measured source-to-background ratios: influence of reconstruction algorithms. Radiother Oncol. 2003;69:247-50.

[30] Ollers M, Bosmans G, van Baardwijk A, Dekker A, Lambin P, Teule J, et al. The integration of PET-CT scans from different hospitals into radiotherapy treatment planning. Radiother Oncol. 2008;87:142-6.

[31] Schafer JL, Graham JW. Missing data: our view of the state of the art. Psychol Methods. 2002;7:147-77.

[32] Cruz JA, Wishart DS. Applications of machine learning in cancer prediction and prognosis. Cancer Inform. 2007;2:59-77.

[33] Fung GM, Mangasarian OL. Proximal support vector machine classifiers. International Conference on Knowledge Discovery and Data Mining. San Francisco (California, USA): ACM; 2001. p. 77-86.

[34] Pepe MS. Receiver Operating Characteristic Methodology. Journal of the American Statistical Association. 2000;95.

[35] Carpenter J, Bithell J. Bootstrap confidence intervals: when, which, what? A practical guide for medical statisticians. Stat Med. 2000;19:1141-64.

[36] Niang N, Saporta G. Resampling ROC curves. Statistics for Data Mining, Learning and Knowledge Extraction. Aveiro (Portugal)2007.

[37] Shariat SF, Capitanio U, Jeldres C, Karakiewicz PI. Can nomograms be superior to other prediction tools? BJU Int. 2009;103:492-5; discussion 5-7.

[38] lasonos A, Schrag D, Raj GV, Panageas KS. How to build and interpret a nomogram for cancer prognosis. J Clin Oncol. 2008;26:1364-70.

[39] Jakulin A, Možina M, Demšar J, Bratko I, Zupan B. Nomograms for visualizing support vector 
$\mathbf{8 0}$ Chapter 4

machines. International Conference on Knowledge Discovery and Data Mining. Chicago (Illinois, USA): ACM; 2005. p. $108-17$

[40] Dodd LE, Pepe MS. Partial AUC estimation and regression. Biometrics. 2003;59:614-23.

[41] Starmans MH, Krishnapuram B, Steck H, Horlings H, Nuyten DS, van de Vijver MJ, et al. Robust prognostic value of a knowledge-based proliferation signature across large patient microarray studies spanning different cancer types. Br J Cancer. 2008;99:1884-90.

[42] Cascini GL, Avallone A, Delrio P, Guida C, Tatangelo F, Marone P, et al. 18F-FDG PET is an early predictor of pathologic tumor response to preoperative radiochemotherapy in locally advanced rectal cancer. J Nucl Med. 2006;47:1241-8. 


\section{Chapter 5 \\ Blood biomarkers are helpful in the prediction of response to chemoradiation in rectal cancer: a prospective, hypothesis driven study on patients with locally advanced rectal cancer}

Jeroen Buijsen ${ }^{\star}$, Ruud G. van Stiphout ${ }^{\star}$, Paul P.C.A. Menheere, Guido Lammering, Philippe Lambin

* Equal contribution 


\section{Purpose/objective}

Chemoradiation (CRT) has been shown to lead to downsizing in an important part of rectal cancers. In order to tailor treatment at an earlier stage during treatment, predictive models are being developed. Adding blood biomarkers may be attractive for prediction, as they can be collected very easily and determined with excellent reproducibility in clinical practice. The hypothesis of this study was that blood biomarkers related to tumor load, hypoxia and inflammation can help to predict response to CRT in rectal cancer.

\section{Material/methods}

295 patients with locally advanced rectal cancer who were planned to undergo CRT were prospectively entered into a biobank protocol (NCT01067872). Blood samples were drawn before start of CRT. Nine biomarkers were selected, based on a previously defined hypothesis, and measured in a standardized way by a certified lab: CEA, CA19-9, LDH, CRP, IL-6, IL-8, CA IX, osteopontin and 25-OH-vit D. Outcome was analyzed in two ways: PCR vs. non-pCR and responders (defined as ypT0-2N0) vs. non-responders (all other ypTN stages).

\section{Results}

276 patients could be analyzed. $20.7 \%$ developed a pCR and $47.1 \%$ were classified as responder. In univariate analysis CEA $(p=0.001)$ and osteopontin $(p=0.012)$ were significant predictors for $\mathrm{PCR}$. Taking response as outcome CEA $(p<0.001), \mathrm{IL}-8(p<0.001)$ and osteopontin ( $p=0.004)$ were significant predictors. In multivariate analysis CEA was the strongest predictor for $\mathrm{pCR}(\mathrm{OR0.92}, \mathrm{p}=0.019)$ and CEA and IL-8 predicted for response (OR 0.97, $p=0.029$ and $O R 0.94, p=0.036$ ). The model based on biomarkers only had an AUC of 0.65 for $\mathrm{PCR}$ and 0.68 for response; the strongest model included clinical data, PET-data and biomarkers and had an AUC of 0.81 for $\mathrm{PCR}$ and 0.78 for response.

\section{Conclusion}

CEA and IL-8 were identified as predictive biomarkers for tumor response and PCR after CRT in rectal cancer. Incorporation of these blood biomarkers leads to an additional accuracy of earlier developed prediction models using clinical variables and PETinformation. The new model could help to an early adaptation of treatment in rectal cancer patients. 


\section{Introduction}

Combined treatment is the cornerstone of rectal cancer treatment. In case of locally advanced rectal cancer, defined as a tumor with a predicted positive circumferential resection margin (CRM) or four or more positive lymph nodes, chemoradiotherapy (CRT) has become standard of care [1]. Pathological complete response (pCR) rates typically lie between 15 and 20\% after CRT [2, 3] depending on the radiotherapy dose given and the interval between CRT and surgery. The group of patients that develop a PCR is of particular interest, because they have a better prognosis [2] and may be offered less invasive surgery [4] or surgery may be completely omitted [5, 6]. Therefore it would be an advantage if the $\mathrm{PCR}$ rate could be increased. There are several possible strategies, of which early response prediction during CRT, leading to treatment adaptation, is very attractive.

In the past, clinical parameters as well as information from PET-scans before and during treatment have been found to be predictive for treatment outcome [7-9]. A prediction model based on tumor length, CT- and cN-stage had a predictive performance of of 0.61 as expressed by the area under the curve (AUC) of the receiver operating characteristic (ROC) curve. A second model including maximal standardized uptake value (SUV) of the tumor derived from a PET-scan before the start of treatment, maximal tumor diameter as measured on PET-scan, tumor location and cN-stage, resulted in an AUC of 0.68 [9]. PETscan after 2 weeks of CRT has been shown to be very predictive for response (tumor regression grade (TRG) 1-2 vs TRG 3-5 according to Mandard) [8].

It is attractive to consider the addition of blood biomarkers to these predictors, since samples can be collected easily, are relatively cheap to measure, and they contain information about different aspects of tumor biology. Furthermore, they can be measured accurately and precisely using standardized methods. Reports on the predictive value of blood biomarkers are limited to studies mainly evaluating 1 or 2 biomarkers [7, 10-13]. The most studied biomarker for response to CRT in rectal cancer is carcinoembryonic antigen (CEA) and one study analyzed the predictive value of osteopontin and interleukin-6. Based on 
these data combined with data of prognostic studies in colorectal cancer and our experience with a blood biomarker model in lung cancer [14], we decided to include 9 biomarkers. CEA and CA19-9 are related to tumor load, interleukin- 6 and -8 (IL-6 and IL-8) and C-reactive protein (CRP) are markers of inflammation, lactate dehydrogenase (LDH) is a marker of cell death, carbonic anhydrase IX (CA IX) and osteopontin are hypoxia markers and 25-OH-vitamin D may induce growth arrest and apoptosis of tumor cells.

In this prospective study we tested the hypothesis that these biomarkers are helpful in the prediction of response to CRT in rectal cancer.

\section{Methods and materials}

\section{Patient population}

All patients who were treated according to the protocol "chemoradiation, rectal cancer, 50.4 Gy" between January 2005 and December 2009 and who gave written informed consent for inclusion in the biobank protocol (NCT01067872) and from whom blood samples were collected were included in this study. This query resulted in 295 patients. Nineteen patients were ineligible for different reasons ( 1 patient was treated with short course radiotherapy $5 \times 5 \mathrm{~Gy}, 5$ patients were not operated on and entered in a wait\&see study so no ypTN stage could be determined, 2 patients underwent a TEM resection so no ypN stage was available, 6 patients had metastases and were treated with palliative intent, 1 patient died during treatment, in 1 patient all biobank material was hemolytic and in 3 patients the PA report could not be retrieved), resulting in 276 patients for analysis. In 9 patients there were technical problems with the biomarker measurements, so that 267 patients were available for the biomarker analysis. Locally advanced disease was defined as a distal T3 tumor and/or N2 status and/or a mid- or upper-rectal tumor with a predicted circumferential resection margin $<2 \mathrm{~mm}$, or any T4 tumor. Locoregional staging for clinical tumor and nodal stage was based on MRI. In 198 patients for whom biomarkers were available a PET-CT was made for radiotherapy planning. Treatment consisted of 28 fractions of $1.8 \mathrm{~Gy}$ on the primary tumor, mesorectum, presacral area and external iliac 
lymph nodes in combination with capecitabine $825 \mathrm{mg} / \mathrm{m}^{2}$ BID. Fourteen patients did not receive a dose of $50.4 \mathrm{~Gy}$. In 12 patients treatment had to be discontinued because of toxicity and in 2 patients a lower total dose was prescribed because of radiotherapy in the past. Patients were operated on 8-10 weeks after the end of CRT. Pathology reports were collected from the referring hospitals.

\section{Blood samples}

Blood samples were collected before the start of treatment. Samples were processed and stored using a standard protocol. All biomarkers were measured in serum, except for osteopontin and CA IX, which were measured in EDTA plasma. Biomarker measurements were done in one single, certified laboratory, using commercially available kits. All samples were analyzed simultaneously. Measurements were performed using the following kits: CEA was measured using a solid-phase, two-site sequential chemoluminescent immunometric assay (Siemens Medical Solutions Diagnostics, LA, USA), IL-6 and IL-8 were determined with a solid phase, enzyme labeled, chemoluminescence sequential immunometric assay (Siemens Medical Solutions Diagnostics, LA, USA). (LDH (Beckman Coulter, Fullerton, CA), CRP (Beckman, Coulter Fullerton, CA) CA 19-9 has been determined on Brahms Kryptor (Brahms, ThermoFisher, Hennigsdorf, Germany) with a sandwich immuno-fluorescent assay. 25-OH-Vitamine-D was measured with a commercially available radioimmunoassay (IDS, Frankfurt am Main, Germany). CA IX is measured by an enzyme-linked immunosorbent assay (Wilex (OncogenScience), Cambridge, MA, USA), and OPN was measured by an enzyme-linked immunosorbent assay (Quantikine Human Osteopontin Immuno assay; R\&D Systems, Minneapolis, MN, USA). OPN, 25-OH-vitamin-D and CA IX were measured using manual methods in duplicate. All other biomarkers were measured in singletons.

\section{Statistical analysis}

Primary endpoint in this study was PCR defined as the absence of any tumor cells in the operative pathologic specimen, at the primary site, or in lymph node regions. It was hypothesized that the addition of biomarkers would lead to an 
increase of the AUC from 0.7 to 0.8. Assuming a correlation between the models of 0.6 a sample of 57 patients with PCR and 219 without pCR achieves a power of 0.74 to detect a difference of 0.1 between the model with and without biomarkers. An alternative endpoint was good responders, defined as ypT02N0, versus poor responders, defined as ypT3 and/or ypN1-2. Missing values were completed using expectation-maximation imputation. Correlations between biomarkers were analyzed by calculating Spearman's rho. Because the biomarkers showed a skewed distribution, the Mann-Whitney $U$ test was used to determine significant univariate predictors of response. Logistic regression analysis was used to identify the significant multivariate predictors of response. The next step was to combine blood biomarkers with clinical data and PET parameters. For this analysis clinical and PET-variables were selected manually, based on an earlier predictive model for PCR [9]. The included clinical variables were tumor length, clinical T and $\mathrm{N}$ stage, all based on MRI. Included PETfeatures were maximum SUV, pretreatment metabolic volume and maximum diameter. The two latter variables were measured using a source-tobackground ratio method as has been described earlier [15]. ROC curves were constructed and the AUC was calculated. In order to approximate the true AUC and calculate confidence intervals bootstrapping $(n=1000)$ was used. A perfect prediction model results in an AUC of 1.0, while an AUC of 0.5 indicates that response is predicted correctly in $50 \%$ of cases (i.e. as good as chance). Statistical analyses were performed using Matlab, release 2010b (The MathWorks, Natick, MA).

\section{Ethics}

The biobank study was conducted according to the Dutch law and approved by the local medical ethics committee. All patients gave written informed consent before collection of the blood samples. 


\section{Results}

The patient characteristics are shown in table 1 . The majority of patients had a tumor penetrating through the bowel wall and predicted positive lymph nodes on MR. In the total database $20.7 \%$ of patients developed a pCR and $47.1 \%$ of patients were classified as responder. Table 2 shows the results of the biomarker measurements for the different outcome groups, as well as the PET-parameters that were included in the model. In general lower serum levels for blood biomarkers were seen in the poor responding groups (except for 25-OH-vitamin-D). Additional analysis (not shown in the table) revealed significant positive correlations between IL-6, IL-8, CRP and osteopontin and between CEA and CA19-9.

Table 1 Patient characteristics $(\mathrm{N}=276)$

\begin{tabular}{llll}
\hline Characteristic & & N & {$[\%]$} \\
\hline Age [years] & median & 65.8 & \\
Gender & range & $23.0-92.2$ & \\
& male & 179 & {$[64.9]$} \\
Clinical tumor stage (cT) & female & 97 & {$[35.1]$} \\
& 2 & 26 & {$[9.4]$} \\
Clinicalnodal stage (cN) & 3 & 207 & {$[75.0]$} \\
& 4 & 43 & {$[15.6]$} \\
WHO performance index & 0 & 47 & {$[17.0]$} \\
& 1 & 114 & {$[41.3]$} \\
Tumor length [cm] & 2 & 115 & {$[41.7]$} \\
& 1 & 218 & {$[79.0]$} \\
pCR (ypTON0) & 2 & 53 & {$[19.2]$} \\
& median & 5 & {$[1.8]$} \\
Good response (ypT012N0) & range & 5.0 & \\
& No & $2.0-13.0$ & \\
& Yes & 57 & {$[79.3]$} \\
& No & 146 & {$[20.7]$} \\
& Yes & 130 & {$[47.9]$} \\
\hline
\end{tabular}

\section{Univariate analysis}

Univariate analysis indicated that CEA and osteopontin were significant predictors for $\operatorname{pCR}(p=0.001, p=0.012$ respectively) and that CEA, IL-8 and 
osteopontin were significant predictors for response $(p<0.001, p<0.001, p=0.004$ respectively) as shown in table 3 . Lower serum levels of these markers correlated with a higher chance of response to chemoradiation. Of the clinical parameters clinical $\mathrm{N}$-stage was predictive for $\mathrm{pCR}(p=0.026)$ and response $(p=0.001)$ in univariate analysis and tumor length $(p=0.02)$ and clinical T-stage $(p=0.004)$ for response only. The pre-treatment metabolic volume and maximum diameter based on PET were predictive for both outcome measures ( $p=0.016$ and 0.009 for $\mathrm{pCR}$ and $p=0.006$ and 0.005 for response respectively).

Table 2 Biomarker levels and PET parameters (average \pm standard deviation) compared for the subpopulations of pCR vs no pCR and good response vs no good response

\begin{tabular}{llcccc}
\hline & & \multicolumn{2}{c}{ PCR (ypTON0) } & \multicolumn{2}{c}{ Good response (ypT012N0) } \\
& & $n o$ & yes & $n o$ & yes \\
\hline Biomarkers & CEA & $14.7 \pm 28.4$ & $8.7 \pm 22.9$ & $18.1 \pm 33$ & $8.5 \pm 18.5$ \\
$(\mathrm{~N}=267)$ & IL-6 & $4.0 \pm 8.4$ & $2.9 \pm 2.7$ & $4.8 \pm 10.1$ & $2.7 \pm 2.4$ \\
& IL-8 & $15.8 \pm 9$ & $14.0 \pm 7.4$ & $17.3 \pm 10$ & $13.4 \pm 6.4$ \\
& LDH & $181.3 \pm 43.8$ & $176.2 \pm 33.9$ & $181.9 \pm 46.7$ & $178.5 \pm 36.1$ \\
& CRP & $11.5 \pm 24.5$ & $7.8 \pm 8$ & $13.8 \pm 29.1$ & $7.5 \pm 9.2$ \\
& CA 19-9 & $26.9 \pm 29.1$ & $25.1 \pm 31.9$ & $28.3 \pm 28.8$ & $24.6 \pm 30.6$ \\
& vitD-25 & $53.2 \pm 20.9$ & $55.5 \pm 18.8$ & $52.6 \pm 22.4$ & $54.8 \pm 18.2$ \\
PET parameters & CA-9 & $282.5 \pm 275$ & $274.4 \pm 302.9$ & $282.0 \pm 238.4$ & $279.6 \pm 320.9$ \\
$(N=198)$ & OPN & $79.2 \pm 28.9$ & $68.2 \pm 16$ & $81.6 \pm 30.9$ & $72 \pm 21.3$ \\
& SUV & $15.3 \pm 6.3$ & $13.6 \pm 5.2$ & $14.3 \pm 5.6$ & $15.6 \pm 6.7$ \\
& MTV [cc] & $33.8 \pm 33.5$ & $25 \pm 20.2$ & $37.6 \pm 39.1$ & $25.7 \pm 17.6$ \\
& Max diameter & $6.5 \pm 1.9$ & $5.8 \pm 1.6$ & $6.7 \pm 2.1$ & $5.9 \pm 1.4$ \\
& [Cm] & & & & \\
\hline
\end{tabular}

\section{Multivariate biomarker model}

Table 3 shows the results of the multivariate analysis for the total set of parameters as well as a selection of biomarkers and clinical and PETparameters. In the complete set of parameters IL-8 was the only significant predictor for response $(p=0.05)$, while osteopontin was borderline significant for $\mathrm{pCR}$ prediction $(\mathrm{p}=0.056)$. As a next step a manual selection of the most promising predictors was made. Blood biomarkers that had a significant 


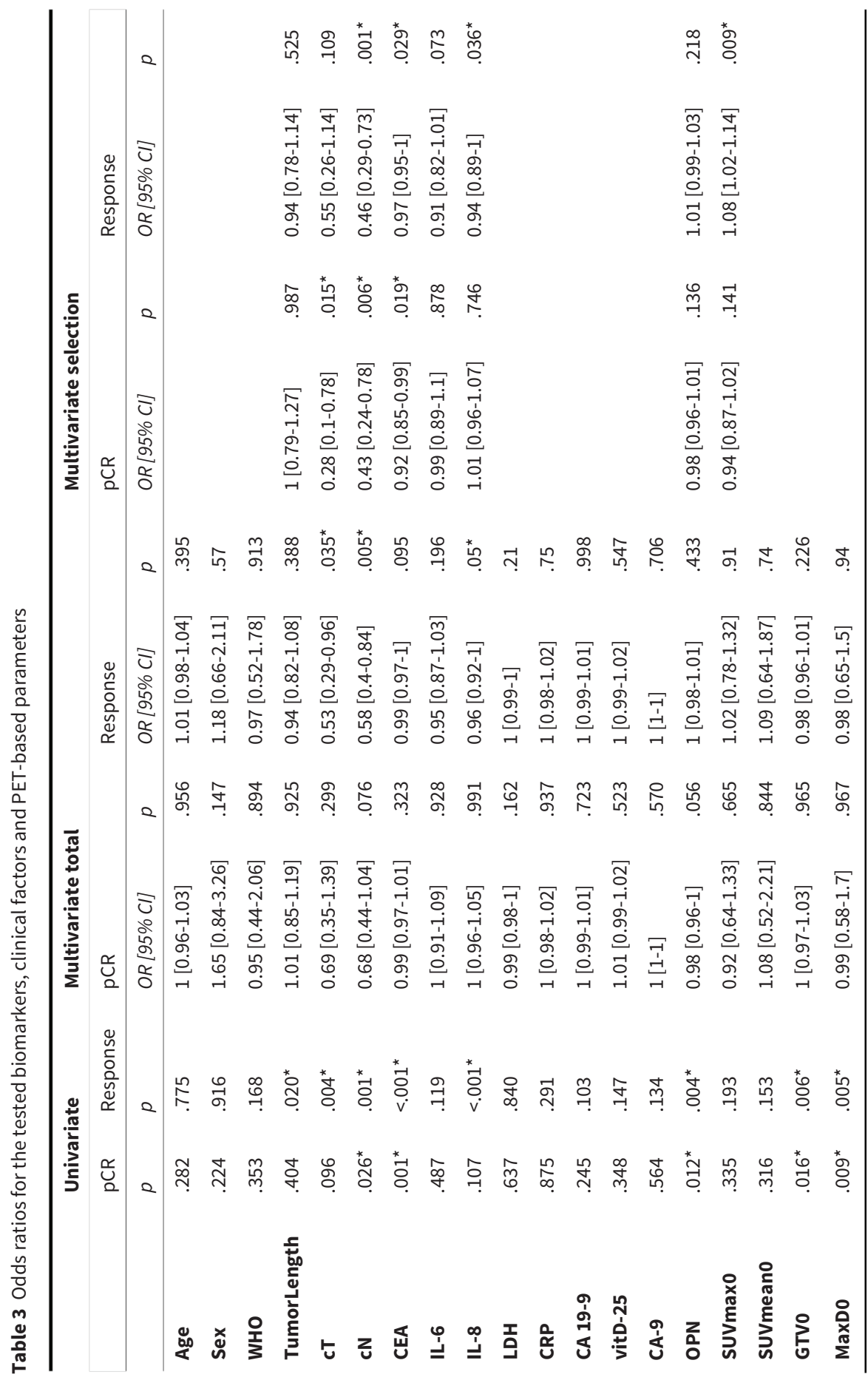


predictive value in either univariate or multivariate analysis were included and IL-6, although not significant, was included because it had a predictive value in a prognostic model for lung cancer [14]. In this selection of biomarkers consisting of CEA, IL-6, IL-8 and osteopontin, CEA was the only significant predictor of $\mathrm{pCR}$ in multivariate analysis $(p=0.019)$ and CEA and IL- 8 significantly predicted response ( $p=0.029, p=0.021$ respectively). Including all biomarkers resulted in an AUC of $0.65(95 \% \mathrm{Cl} 0.57-0.73)$ for $\mathrm{PCR}$ prediction and 0.68 (95\% 0.61-0.75) for response prediction. Table 3 (supplementary data) shows the odds ratios for all tested biomarkers as well as the clinical and PET-based parameters.

\section{Combination of blood biomarkers with clinical and PET data}

The biomarker selection was then added to the parameters that were predictive for response in an externally validated prediction model based on clinical and PET-scan data [9]. The final model consisted of eight variables: tumor length, clinical T stage, clinical N stage, CEA, IL-6, IL-8, osteopontin, and maximal SUV on PET before start of treatment. In the current dataset tumor length was not a significant predictor for response to chemoradiation, but $\mathrm{CT}$ and $\mathrm{CN}$ were. Maximal SUV was only predictive for response, not for $\mathrm{PCR}$.

In figure 1 the ROC curves for the combined models based on biomarkers and clinical data (figure $1 \mathrm{~A}$ and $1 \mathrm{a}$ ) and biomarkers, clinical data and PET information (figure $1 B$ and 1 b) are depicted, as well as the resulting AUCs (figure $1 \mathrm{C}$ and $1 \mathrm{C}$ ). The model based on biomarkers only resulted in an AUC that was more or less comparable to the clinical model. The AUC of the clinical model was 0.64 (95\% Cl 0.56-0.71) for pCR and 0.66 (95\% Cl 0.60-0.72) for response.

Combining clinical parameters and biomarkers (AUC 0.69 (95\% Cl 0.62-0.77) for pCR and 0.73 (95\% Cl 0.65-0.79) for response) made the model stronger than the model based on biomarkers only or clinical data only. This effect was most pronounced for prediction of response. Addition of information from a PET-scan acquired before the start of treatment leads to the strongest models for the prediction of both $\mathrm{pCR}$ and response, resulting in AUCs of $0.81(95 \% \mathrm{Cl} 0.73$ $0.88)$ for $\mathrm{pCR}$ and 0.78 (95\% $\mathrm{Cl} 0.71-0.85)$ for response. 
PCR prediction
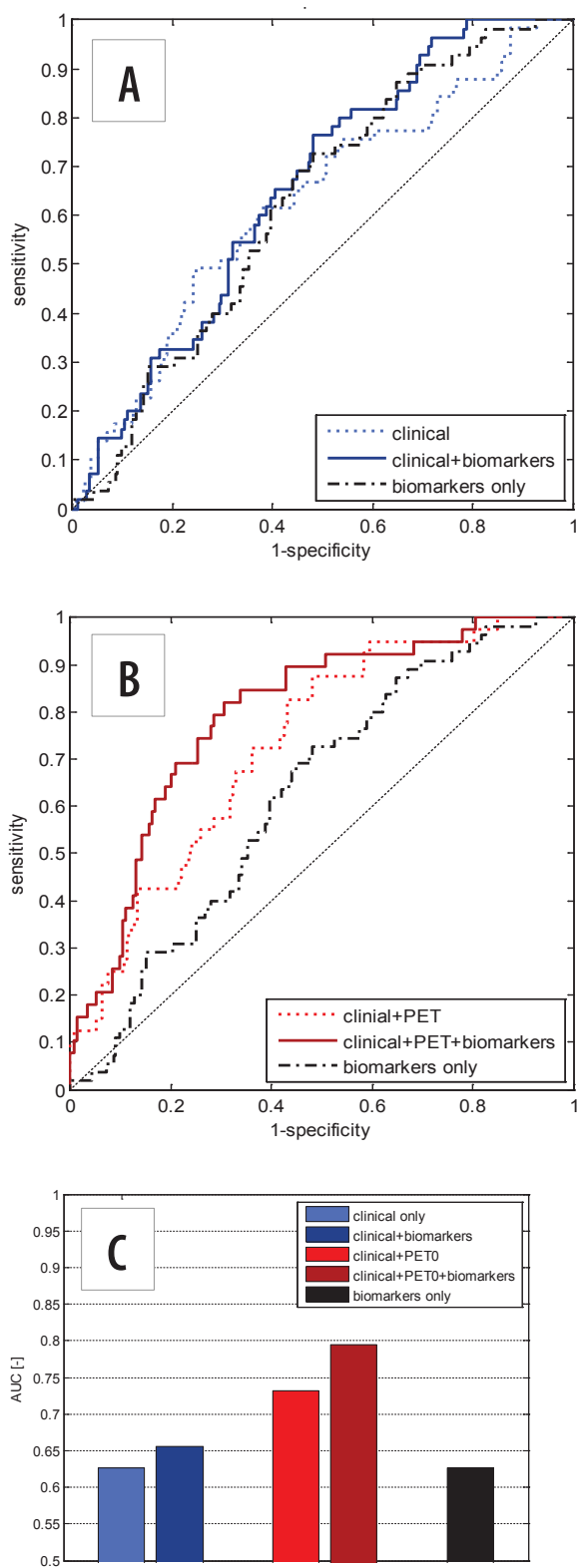

Response prediction
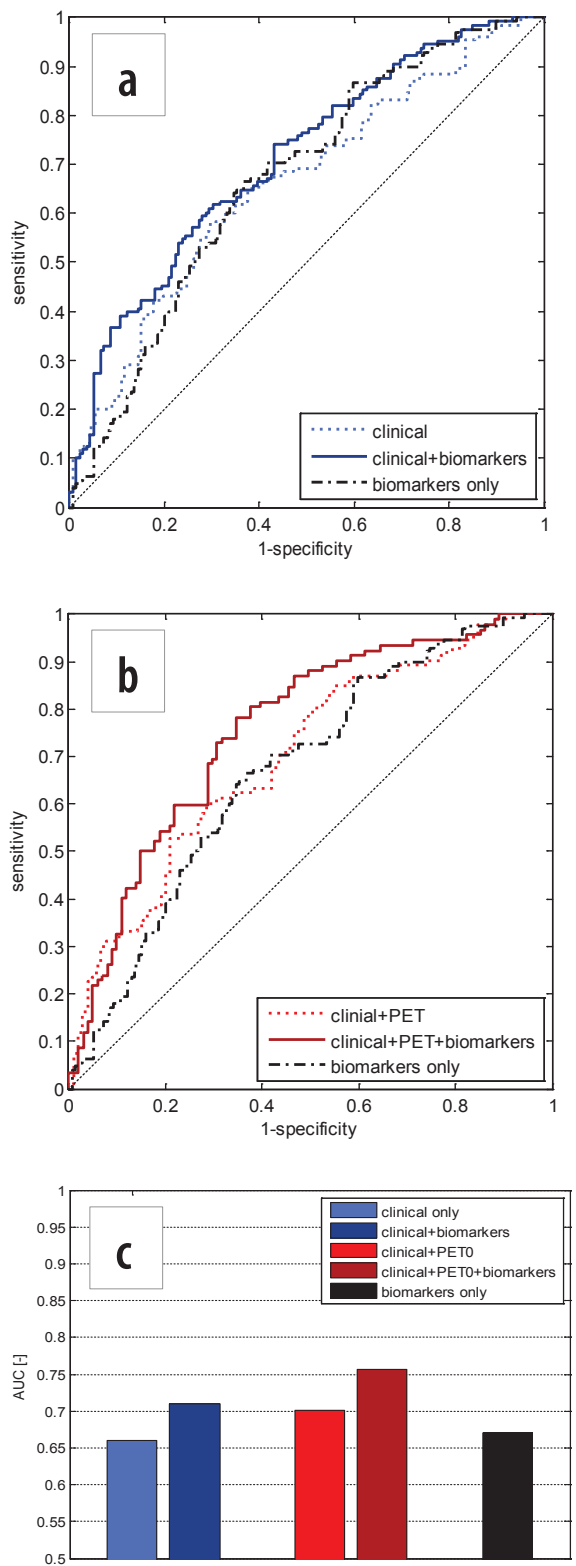

Figure 1 ROC curves for prediction models for $\mathrm{PCR}$ and response including biomarkers and clinical variables ( $A$ and $a$ ) and biomarkers, clinical variables and PET-data ( $B$ and $b$ ) and resulting AUC for the different prediction models ( $\mathrm{C}$ and $\mathrm{C}$ ) 
The data of the combined prediction model was used to build a nomogram, which is depicted in figure 2. This nomogram can be used online at www.predictcancer.org. Because this nomogram has not yet been externally validated, it should be regarded as hypothesis generating.

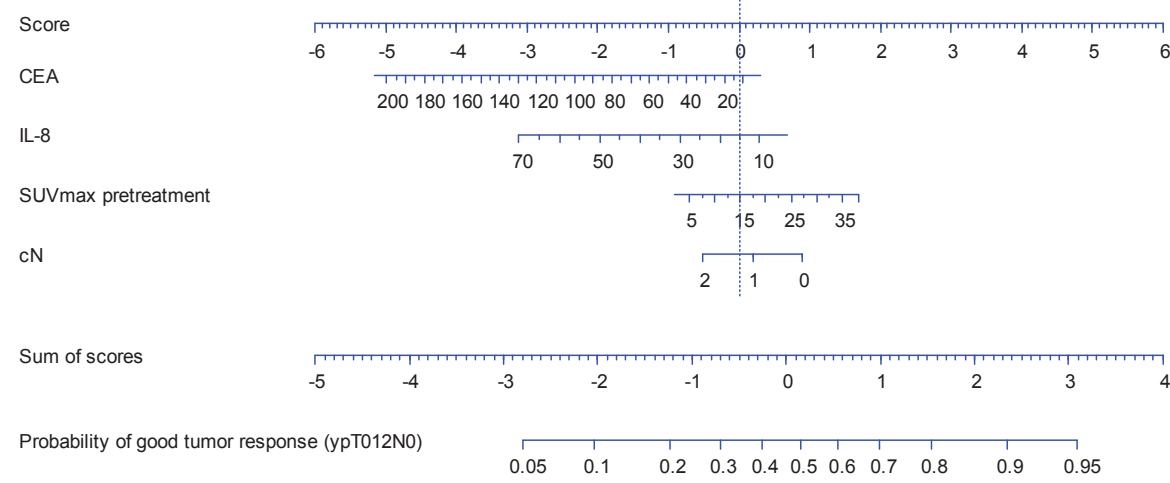

Figure 2 Nomogram for response prediction. A score for each predictor can be read out at the top scale (Score). All summed scores (Sum of scores scale) can be converted directly to the probability of response.

\section{Discussion}

To the best of our knowledge this is the first prospective study evaluating the predictive value of a broad range of blood biomarkers analyzed in a standardized way for response prediction to CRT in rectal cancer. CEA turned out to be a significant predictor for PCR and CEA and IL-8 were predictive for response. Including these blood biomarkers in models based on clinical parameters and PET-based parameters resulted in an increased performance of the prediction models.

Osteopontin is a glycophosphoprotein secreted by different cell types, that has been shown to be associated not only with malignancies but also with acute and chronic inflammatory processes. It influences adhesion, migration, invasion, chemotaxis and cell survival [16] and is a marker of tumor aggressiveness and early progression [17]. Although the role of osteopontin as a 
prognostic factor has been studied extensively in a broad range of solid tumors, only one study looked at the predictive value of osteopontin levels and response to CRT in rectal cancer. Debucquoy et al. found an association between lower osteopontin levels and better response in 30 rectal cancer patients, which is in line with our findings, but this did not reach statistical significance [13]. In our patient group it was a significant predictor in univariate analysis, but it lost significance in multivariate analysis. This could be explained by the fact that osteopontin and IL-8 showed a positive correlation.

CEA is an antigen produced by the normal fetus and only in very low concentrations by normal cells of the adult body. In the tumor environment it plays a role in intercellular recognition and attachment. It has been shown to be of prognostic value in colorectal cancer independent of clinical stage and differentiation grade [18]. Although preoperative CEA levels cannot be used to make treatment decisions in colorectal cancer, they consistently have a prognostic value. Patients having a CEA level $>5 \mu \mathrm{g} / \mathrm{l}$ have a significant worse disease free and overall survival than patients with lower CEA levels. Three studies looked at the predictive value of CEA levels for response to CRT in rectal cancer. Das et al studied a group of 562 rectal cancer patients treated with CRT [7]. In their patient group CEA was only predictive for $\mathrm{pCR}$ in univariate analysis. However, they chose to dichotomize CEA levels (below and above $2.5 \mu \mathrm{g} / \mathrm{l}$ ), while in our model CEA was incorporated as a continuous variable. Yoon et al did an analysis in a group of 351 rectal cancer patients [12]. In multivariate analysis CEA levels $\leq 5 \mu \mathrm{g} / \mathrm{l}$ were predictive for downstaging and complete regression. Park et al did a retrospective analysis in 352 rectal cancer patients [11]. CEA was analyzed as a continuous variable and had a significant predictive value for responders vs. non-responders. A higher CEA was an independent predictor of poor response to CRT and a worse disease free survival.

IL-8 is a pro-inflammatory chemokine that plays a role in attracting neutrophils. Through the activation of phosphatidyl-inositol-3-kinase (PI3-K) and phospholipase $\mathrm{C}$ it can activate the Akt/mTOR and Raf/Mek/Erk pathways, leading to the promotion of angiogenesis, proliferation and survival and the migration of cancer cells [19]. Polymorphisms in IL-8 have been described to be 
related to an increased risk of recurrence in rectal cancer [20] and the risk of nodal involvement [21], indicating a possible relationship with tumor biology of IL-8 in rectal cancer. However, until now there are no reports of a potential value of IL-8 in the prediction of response to CRT.

In this study response was measured in two ways: pCR and ypT0-2N0. Of these 2 endpoints $\mathrm{PCR}$ is the most robust, although the definition of $\mathrm{PCR}$ can be difficult. In this cohort routine pathological examination was performed and reported in a standardized way. A recent comparison between routine pathological examination and additional step sections in resection specimens showing no viable tumor cells at initial examination, showed no differences in outcome [22]. Furthermore, pooled analysis of a large series of patients included in different studies, showed a clear prognostic value of pCR after CRT for long-term outcome, even if pooled from different studies, indicating that PCR as scored in routine pathology procedures is a valuable endpoint [2]. The most frequently used method to distinguish responders from non-responders is by means of a tumor regression grade (TRG). However, for this cohort TRG was not scored routinely and we chose to use ypT0-2N0 as definition of response. It makes sense to predict the group of good responders, because they could be suitable for less invasive surgery, like transanal resection or TEM-surgery.

Blood biomarkers give information about tumor biology in an indirect way. A more direct insight can be gained from genetic alterations within the tumor. An overview of these molecular biomarkers is given in the review of Kuremsky et al [23]. A possible problem related to molecular biomarkers is the heterogeneity in tumors, making it necessary to collect a representative sampling of tumor material and the invasive procedure needed to collect material. Blood biomarkers have the advantage that they are easy to collect and that they provide information about the "average" tumor. This makes blood biomarkers useful for daily practice. However translation of the results of this study should be done cautiously, because all biomarkers in this study were measured in a standardized way in one single laboratory, using the same kits. Less thorough and sensitive procedures might influence negatively the added value of biomarkers. 
Ideally response prediction takes place before the start of a treatment, so that a patient can be offered the treatment with the highest success rate. In the case of response prediction for rectal cancer, clinical factors and pre-treatment PETscan have been shown to have predictive value before the start of treatment [9], but the performance of predictive models based exclusively on pre-treatment data typically lies between 0.65 and 0.70 . The predictive value of biomarkers only is in the same range, but the combination of biomarkers and other pretreatment data results in a stronger prediction model.

The data presented here can be seen as a proof of principle that biomarkers contain predictive information for rectal cancer, but external validation of this prediction model is necessary for a better estimation of the performance and reproducibility of the model [24]. For use in clinical practice a stronger performance is desirable. A possibility to strengthen the predictive model is to incorporate response data obtained early during CRT. If this time point lies early in the treatment course, it is still possible to modify treatment. For PET-CT it has already been shown that changes in glucose metabolism after 2 weeks of CRT resulted in a stronger prediction model [8]. An intriguing question is whether early changes in blood biomarker levels during CRT can further enhance the performance of this model. This question will be subject of future research.

In conclusion, pre-treatment CEA levels help to predict $\mathrm{PCR}$ after CRT for rectal cancer and CEA and IL-8 levels are helpful in the prediction of response to CRT. These blood biomarkers have added value to earlier published prediction models based on pre-treatment clinical- and PET-data and can be used in decision support systems for tailored therapy [24]. 


\section{References}

[1] Valentini, V, Aristei, C, Glimelius, B, et al. Multidisciplinary Rectal Cancer Management: 2nd European Rectal Cancer Consensus Conference (EURECA-CC2). Radiother Oncol 2009;92:148-163.

[2] Maas, M, Nelemans, PJ, Valentini, V, et al. Long-term outcome in patients with a pathological complete response after chemoradiation for rectal cancer: a pooled analysis of individual patient data. Lancet Oncol 2010;11:835-844.

[3] Zorcolo, L, Rosman, AS, Restivo, A, et al. Complete Pathologic Response after Combined Modality Treatment for Rectal Cancer and Long-Term Survival: A Meta-Analysis. Ann Surg Oncol 2012.

[4] Bokkerink, GM, de Graaf, EJ, Punt, CJ, et al. The CARTS study: Chemoradiation therapy for rectal cancer in the distal rectum followed by organ-sparing transanal endoscopic microsurgery. BMC Surg 2011;11:34.

[5] Maas, M, Beets-Tan, RG, Lambregts, DM, et al. Wait-and-see policy for clinical complete responders after chemoradiation for rectal cancer. J Clin Oncol 2011;29:4633-4640.

[6] Glynne-Jones, R, Hughes, R. Critical appraisal of the 'wait and see' approach in rectal cancer for clinical complete responders after chemoradiation. Br J Surg 2012;99:897-909.

[7] Das, P, Skibber, JM, Rodriguez-Bigas, MA, et al. Predictors of tumor response and downstaging in patients who receive preoperative chemoradiation for rectal cancer. Cancer 2007;109:1750-1755.

[8] Janssen, $\mathrm{MH}$, Ollers, MC, van Stiphout, RG, et al. PET-based treatment response evaluation in rectal cancer: prediction and validation. Int J Radiat Oncol Biol Phys 2012;82:871-876.

[9] van Stiphout, RG, Lammering, G, Buijsen, J, et al. Development and external validation of a predictive model for pathological complete response of rectal cancer patients including sequential PET-CT imaging. Radiother Oncol 2011;98:126-133.

[10] Lu, K, Zhu, Y, Sheng, L, Liu, L, Shen, L, Wei, Q. Serum fibrinogen level predicts the therapeutic response and prognosis in patients with locally advanced rectal cancer. Hepatogastroenterology 2011;58:1507-1510.

[11] Park, JW, Lim, SB, Kim, DY, et al. Carcinoembryonic antigen as a predictor of pathologic response and a prognostic factor in locally advanced rectal cancer patients treated with preoperative chemoradiotherapy and surgery. Int J Radiat Oncol Biol Phys 2009;74:810-817.

[12] Yoon, SM, Kim, DY, Kim, TH, et al. Clinical parameters predicting pathologic tumor response after preoperative chemoradiotherapy for rectal cancer. Int J Radiat Oncol Biol Phys 2007;69:1167-1172.

[13] Debucquoy, A, Goethals, L, Geboes, K, Roels, S, Mc Bride, WH, Haustermans, K. Molecular responses of rectal cancer to preoperative chemoradiation. Radiother Oncol 2006;80:172-177.

[14] Dehing-Oberije, C, Aerts, H, Yu, S, et al. Development and validation of a prognostic model using blood biomarker information for prediction of survival of non-small-cell lung cancer 
patients treated with combined chemotherapy and radiation or radiotherapy alone (NCT00181519, NCT00573040, and NCT00572325). Int J Radiat Oncol Biol Phys 2011;81:360-368.

[15] Buijsen, J, van den Bogaard, J, Janssen, MH, et al. FDG-PET provides the best correlation with the tumor specimen compared to MRI and CT in rectal cancer. Radiother Oncol 2011;98:270276.

[16] Anborgh, PH, Mutrie, JC, Tuck, AB, Chambers, AF. Role of the metastasis-promoting protein osteopontin in the tumor microenvironment. J Cell Mol Med 2010;14:2037-2044.

[17] Weber, GF, Lett, GS, Haubein, NC. Osteopontin is a marker for cancer aggressiveness and patient survival. Br J Cancer 2010;103:861-869.

[18] Goldstein, MJ, Mitchell, EP. Carcinoembryonic antigen in the staging and follow-up of patients with colorectal cancer. Cancer Invest 2005;23:338-351.

[19] Waugh, DJ, Wilson, C. The interleukin-8 pathway in cancer. Clin Cancer Res 2008;14:6735-6741.

[20] Gordon, MA, Gil, J, Lu, B, et al. Genomic profiling associated with recurrence in patients with rectal cancer treated with chemoradiation. Pharmacogenomics 2006;7:67-88.

[21] Kalady, MF, Coffey, JC, Dejulius, K, Jarrar, A, Church, JM. High-throughput arrays identify distinct genetic profiles associated with lymph node involvement in rectal cancer. Dis Colon Rectum 2012;55:628-639.

[22] Park, SY, Chang, HJ, Kim, DY, et al. Is step section necessary for determination of complete pathological response in rectal cancer patients treated with preoperative chemoradiotherapy? Histopathology 2011;59:650-659.

[23] Kuremsky, JG, Tepper, JE, McLeod, HL. Biomarkers for response to neoadjuvant chemoradiation for rectal cancer. Int J Radiat Oncol Biol Phys 2009;74:673-688.

[24] Lambin, P, van Stiphout, RG, Starmans, MH, et al. Predicting outcomes in radiation oncologymultifactorial decision support systems. Nat Rev Clin Oncol 2012. 
Chapter 5 


\section{Chapter 6 \\ What biochemical and molecular biological factors have greater relevance to treatment decisions?}

Jeroen Buijsen and Guido Lammering

book chapter from:

Multidisciplinary management

of rectal cancer

2012, 41-8, Valentini et al (eds) 
All current treatments for rectal cancer come with a price to pay, namely, the risk for over- and undertreatment based on current treatment decisions leading to sometimes even unnecessary toxicities. Thus, it would further improve the results of both treatment outcome and quality of life, if we could better tailor the treatment for rectal cancer based on validated biochemical and molecular biological factors. It is clear that several already known tumor biological features have an important impact on the individual tumor responses to certain treatments. Thus, detection and analysis of these biomarkers will ultimately lead to better treatment decisions in all parts of the current treatment algorithm. However, most studies so far have been rather limited in its attempts to identify biomarker based predictors for e.g. response to CRT, surgical decisions and systemic therapy. Six genetic biomarkers were identified with the potential of being predictive in the outcome after CRT, like p53, EGFR, TYMS, Ki-67, p21 and bax-bcl-2. TYMS expression measurements seem to be worthwhile to further study in future trials, while survivin showed mostly conflicting results and gene expression profiles with microarrays need further validation with larger patient groups. Nevertheless, there is great interest in the identification of biochemical and molecular biological factors in treatment decision making and many promising developments are on the way, like whole genome DNA, RNA analysis, multiple mutation testing, full sequencing and the association of genotype with phenotype. However, before the first successful biomarker development can been finalized and implemented clinically, several collaborations and consecutively external validations still need to be completed. 


\section{Introduction}

The current standard therapy for rectal cancer in most countries around the world consists of a preoperative treatment either with long-term radiotherapy (RT) and a 5-FU based chemotherapy (CT) or a short course RT followed by surgery or surgery alone depending on the pretherapeutic staging resulting in a 5 -year cumulative rate of local relapse of less than $10 \%$ and an incidence of distant metastases of about $35 \%[1,2]$. The preoperative treatment has a major impact on local control, however with only minimal impact on survival and disease-free survival. So far, no clear evidence has been produced for the benefit of adjuvant chemotherapy, leading to different protocols in different countries and regions around the world. Generally, all parts of the current standard treatment of rectal cancer have its toxicity profiles. Preoperative chemoradiotherapy (CRT) can lead to diarrhea, radiation dermatitis and increased risk for anastomotic leakage and perineal wound complications [3] while short course radiotherapy generally increases the risk for fecal incontinence, anal blood loss, mucus loss and erectile dysfunction [4-6]. The surgical treatment itself is generally accompanied with an increased risk for urinary, bowel and erectile dysfunction, anastomic leakage, perineal wound complications and a mortality rate of $4-11 \%$ depending on age and comorbidity [7-9]. Thus, all treatments come with a price to pay, namely the risk for over- or undertreatment based on the current imaging-related treatment decisions leading to probably unnecessary toxicities and subsequently impact on quality of life. However, rectal cancer is a biological tumor, characterized by several biological features, which are nowadays better and better understood. Dramatic technical improvements in detection assays have made it possible to characterize more and more biochemical and biological features and phenotypes related to individual rectal cancer patients. Thus, biological markers might be able to better tailor the treatment of rectal cancer, thereby minimizing the risk for over- and undertreatment with also an improvement in outcome and quality of life. However, this needs prospective evaluation and stringent validation. It is clear that there is a biological basis for the different responses of individual tumors to certain treatments, like CRT, RT, and CT. The 
tumor microenvironment, e.g. angiogenesis and hypoxia, repopulation and the intrinsic radiosensitivity are currently the best known biological tumor phenotypes, which undoubtly have an important impact on the degree of response to anti-tumor treatments [10].

This chapter will focus on the question where we are now in the detection and analysis of biochemical and biological factors influencing treatment decisions and how far we have already come in our attempts to better individualize treatment based on biomarkers.

\section{Biochemical and molecular biological factors for radiochemotherapy}

The combination of chemotherapy and radiotherapy leads to some degree of pathological downstaging in approximately $40-60 \%$ of patients. The percentage of patients developing a pathological complete response ( $\mathrm{PCR}$ ) varies, but lies typically around $20 \%$. In treatment decision making it would be very helpful to identify good and poor responders in order to give a tailored treatment. Until now the predictive value of several biomarkers has been studied. In these studies various endpoints have been used, but the most common endpoints are overall survival, tumor regression grade (TRG), pCR and downstaging. Kuremsky et al published a review evaluating the potential of genetic biomarkers in predicting the outcome of locally advanced rectal cancer patients treated with chemoradiation [11]. In their review they focused on gene products with more than 5 studies in the literature. The 6 biomarkers that met this criterion were p53, epidermal growth factor receptor (EGFR), thymidylate synthase (TYMS), Ki67, p21 and bax/bcl-2.

The most studied marker is p53, a gene that plays a key role in apoptosis, tumorigenesis and sensitivity to chemotherapeutic agents. Twenty-one studies studying the predictive value of p53 status were identified. Seventeen of these studies could not identify a correlation between p53 expression and tumor response. Of the 4 studies that did show a relationship between p53 and outcome, 3 identified a p53 mutation as a predictor of poor response, whereas the other study found a positive predictive value for mutated p53. Therefore, 
p53 does not seem to be a good predictor for tumor response to chemoradiation.

EGFR is important in the regulation of various cellular responses, like proliferation, apoptosis and differentiation. Overexpression of EGFR has been shown in $60-80 \%$ of all colorectal cancers and has been associated with a poor prognosis independent of lymph node status. Five studies have been published studying the value of EGFR expression as response predictor. These were studies looking at chemoradiation only, without addition of EGFR inhibitors. Although these studies looked at different end-points, the studies that found a correlation all point towards a better response to chemoradiation in tumors with low EGFR expression. It seems to be more important to quantify the level of EGFR expression rather than divide tumors into positive or negative. One study identified a single nucleotide polymorphism that may be useful as a biomarker for response.

Thymidylate synthase is an important enzyme in DNA synthesis and is the main target of 5-fluorouracil (5-FU). Overexpression of this enzyme leads to 5-FU resistance. Nine studies were identified in the Kuremsky review analyzing the influence of TYMS expression and SNPS in the TYMS enhancer region on tumor response and very recently 2 additional studies have been published. Four studies used immunohistochemical staining and 5 studies used PCR for genotyping. Three studies did not show a correlation between TYMS and tumor response. The remaining 6 studies found in general a better response to chemoradiation in tumors with low TYMS expression. Two studies also included a group of patients treated with radiotherapy only and as could be expected in this group no correlation between TYMS expression and response was observed. Only one study found an inverse correlation, however this was a small study with only 19 patients, who received both 5-FU and oxaliplatin, which could have confounded the results. Recently, however, two studies found a correlation between high expression of TYMS and better response after CRT for rectal cancer [12, 13]. Although there is not enough evidence to use TYMS in daily practice, incorporation of TYMS expression measurements in chemoradiotherapy trials would be worthwhile. Recently, the results of a phase II study have been 
published in which patients were stratified in a good-risk group (no TYMS overexpression) and a poor-risk group (TYMS overexpression) [14]. The goodrisk group was treated with standard chemoradiation consisting of radiotherapy and continuous 5-FU infusion, while the poor-risk patients received weekly irinotecan in addition to standard chemoradiation. A PCR and tumor downstaging was found in $18.9 \%$ and $64.4 \%$ of good-risk patients respectively and in $35.5 \%$ and $64.5 \%$ of poor-risk patients. However, the combination with irinotecan turned out to cause more toxicity. RFS and OS were comparable between both risk groups. This study is the first attempt to stratify the treatment of rectal cancer patients according to TYMS status. Although further phase III trials are needed, it was remarkable that the proportion of patients showing downstaging was comparable between the good- and poor-risk group and the percentage that developed a pCR was higher in the poor-risk group.

Ki-67 is a proliferation marker, which has not shown a clear relationship with chemo- or radiosensitivity. Thirteen articles have been published about the predictive value of Ki-67, of which only 2 showed a correlation. One study found a better response in tumors with high Ki-67 staining, the other showed an association between low Ki-67 expression and response. Therefore Ki-67 is not a good biological marker in rectal cancer.

The tumor suppression gene p21 is activated by DNA damage and causes cell cycle arrest. Four of 8 studies found a correlation between p21 expression and response to chemoradiation. However, the results of these 4 studies are conflicting. Two studies found better responses or survival in patients with low or negative p21 expression, while the other two studies found the opposite. Based on in vitro studies one would expect better results in tumors with low p21 expression, because p21 suppresses apoptosis in case of DNA damage. The two studies which showed better results in positive p21 tumors used other treatments in addition to radiotherapy and 5-FU which may have confounded the results.

The bax and $\mathrm{Bcl}-2$ proteins are involved in apoptosis. Loss of bax function is correlated with chemoresistance in colorectal cancer and $\mathrm{Bcl}-2$ overexpression has been linked with resistance to different chemotherapeutic agents and inhibition of radiation-induced apoptosis. Three studies evaluated the role of 
bax expression in response to chemoradiation. Only one study found a significant correlation. In that study the percentage of bax-positive tumors was significantly higher in the complete-response group than in the partial-response group (54\% vs. 29\%). Bcl-2 was analyzed in 12 studies, but a correlation with response was found in only one study including only 17 patients.

Two rather small studies have tested the value of microarray in prediction of response. The first study tested 54 genes in 23 patients. A different expression pattern was found between responders and non-responders based on downstaging, but this difference was no longer significant if response evaluation was based on TRG. The negative predictive value (NPV) was $86 \%$ and the positive predictive value (PPV) 78\%. The second study included 43 patients and found 42 genes that were differentially expressed between responders and non-responders based on TRG. The PPV using this gene set was $71 \%$ and the NPV $86 \%$. Although these studies show for the first time that gene expression profiles may be helpful in response prediction, they certainly need further validation with larger patient groups.

Quite recently X-ray-repair-cross complementing 1 (XRCC1) has been identified in 3 studies as a potential useful marker for response prediction in rectal cancer [12, 13, 15]. XRCC1 plays an important role in DNA-repair as it is involved in the base excision repair (BER) pathway. One polymorphism, A399G, has been shown to have a predictive role in radiosensitivity of malignant tumors. The first study included 81 patients with locally advanced rectal cancer treated with chemoradiation. Of the patients with an AG phenotype $47 \%$ showed a major response, as compared to $22 \%$ in the $A A$ and GG phenotype group. The second study analyzed 93 patients treated with chemoradiation for rectal cancer. Genotyping was done on peripheral blood monocytes. They found a better response in $\mathrm{G} / \mathrm{G}$ carriers as compared with G/A carriers (OR 4.180; $p=0.003$ ). In the third study DNA from monocytes of 128 rectal cancer patients was analyzed. In this study no statistical significant association with tumor response was found. In conclusion, results of XRCC1 as a predictor of response in rectal cancer are conflicting.

Another protein that attracted interest in the past years is survivin, an inhibitor of apoptosis [16-18]. Again, results are conflicting. Two recent studies reported 
a correlation between survivin expression and tumor response. Higher survivin expression correlated with worse response to chemoradiation. One of these studies compared survivin expression in pre-treatment biopsies and surgical resection specimens and found a worse survival in patients with tumors expressing high levels of survivin after chemoradiation. However, other studies could not find a correlation between survivin expression and clinical outcome, which underlines the need for further studies.

\section{Biochemical and molecular biological factors for surgical decisions}

The surgical treatment strategy for rectal cancer has dramatically changed over the last years. Coming from a standard radical surgical procedure performed in strictly defined TME protocols with only radical resection being the primary goal, it nowadays changes to a more tailored approach with also the aim to reduce toxicity with better quality of life. The surgical treatment usually takes place initially after diagnosis in early stage cancer or after a neoadjuvant treatment consisting of either radiotherapy alone (5x5 Gy) or RCT. In case of a neoadjuvant treatment, a short-course radiotherapy will usually be followed within 1 to 3 days by an immediate surgical resection of the TME, as has been published by the DUTCH TME trial [19]. Thus, the short-course radiotherapy does not induce any down-sizing or down-staging, since the timing of the followed surgical replacement of the rectum tumor does not allow any tumor shrinkage [4]. However, newer trials have suggested that the short-course preoperative radiotherapy indeed could be used to also downsize and downstage the tumor, if the surgical treatment is postponed by at least 6 to 8 weeks after radiotherapy [20,21]. This strategy might increase the potential for better sphincter- and even organ- sparing surgical treatment techniques etc., as has been already proposed after neoadjuvant chemoradiotherapy in selected patients [22]. However, accurate tumor response assessment is crucial in these modern adaptive surgical strategies after neoadjuvant radio- or radiochemotherapy. Biomarkers could help in the treatment decisisons for less aggressive conservative surgical treatments, beside modern imaging and 
clinical examinations. But this applies not only for preoperatively pretreated rectal cancer patients, but also for initially diagnosed patients, in which early stage disease is suggested. These patients could benefit from conservative surgery (e.g. TEM) instead of radical surgery, if accurate biomarkers associated with disease progression, particularly mesorectal nodal metastasis, would become available.

One recently published study compared patterns of gene-specific hypermethylations in radically excised rectal cancers with histopathological stage and came to the conclusion, that locus-specific hypermethylation was more prevalent in early- than late-stage disease and that the hypermethylation of two or more of a panel of five tumor suppressor genes was associated with localized disease [23]. Another study by Rasheed et al., assessed the microvessel density (MVD) and the CA9 expression in more than 100 rectal cancer specimen and came to the conclusion that the MVD was higher in more advanced $\mathrm{T}$ and $\mathrm{N}$ stages, whereas the CA9 expression was generally higher in earlier stages [24]. This however has not been validated yet and warrants further evaluation. Generally, the only molecular markers being currently prognostically relevant in rectal cancer are the Deficient Mismatch Repair and possibly the Kras mutation and the Braf mutations. All else, like expression assays, copy number variation tests and even proteomics is experimental and have not shown any validated correlation with prognosis yet. The recently published analysis of the QUASAR study, which mainly analyzed stage II cases showed MMR, Kras or Braf abnormalities in rectal cancer in 1-4\%, 30-35\% and 2-3\% respectively [25]. All three abnormalities had prognostic impact at different levels, with MMR being the strongest marker for prognostic impact. The defective MMR genes were hMLH-1, hMSH-2 PMS-2 and hMSH-6, detected by either immunohistochemistry or microsatellite testing. However, up until now no biomakers have reached the level of clinical relevance which would allow inclusion in surgical decision making. Recently, the first cohort analysis of 20 patients selected for a non- surgical wait and see strategy after RCT has been published with very promising local and distant controls [22]. The decision making for such a non-surgical approach in patients with good to complete 
clinical response after RCT, which is currently only based on clinical and imaging parameters due to the lack of sufficient biomarkers should be stepwise improved with also biochemical and molecular markers, as soon as they have been approved and validated.

\section{Biochemical and molecular biological factors for adjuvant and systemic therapy}

The value of adjuvant chemotherapy in node-positive colon cancer is clear. Since rectal tumors originate in an organ that is in anatomic continuity with the colon and tumors show a similar histology, it is often argued that results of adjuvant trials in colon cancer can be translated to rectal cancers. However, until now, randomized trials in rectal cancer have failed to show a clear benefit of adjuvant chemotherapy [26]. Especially in patients who have a good response after pre-operative chemoradiation the expected gain of adjuvant treatment is small. Recently, data of 5 large rectal cancer trials including chemoradiation have been pooled to build nomograms for the prediction of local and distal recurrence as well as survival. This analysis confirms that in patients showing a good response after chemoradiation the added benefit of adjuvant chemotherapy is small [27].

Biomarkers could be helpful to identify patients who are at higher risk for recurrent disease. When looking at the value of biomarkers, it is important to distinguish prognostic from predictive factors. A prognostic factor gives information about the risk of recurrence of disease irrespective of a certain treatment while a predictive factor predicts the chance that a patient will benefit from a treatment. Of course, a marker can be both prognostic and predictive.

In colorectal cancer, most evidence is available for the prognostic value of mismatch repair (MMR) gene status. Tumors that are MMR deficient have a better prognosis. There is some debate whether MMR status also has a predictive value. However, the proportion of rectal tumors that is MMR deficient is small (around 1\%). Therefore, it is not a useful marker for rectal cancer.

The role of BRAF status seems to have a predictive value, but the debate is going 
on about a possible predictive role. Because the proportion of rectal tumors carrying a BRAF mutation is very low, BRAF does not seem to be a very important biomarker in rectal cancer.

KRAS status also has been shown to be of prognostic value. In about $40 \%$ of all rectal tumors KRAS is mutated. KRAS mutant tumors have a poor prognosis and a higher chance of recurrence. In the QUASAR trial this difference was even more pronounced for tumors located in the rectum as compared to tumors in other parts of the colon. In this trial the reduced risk of recurrence with chemotherapy was comparable between KRAS wild type and KRAS mutated tumors. However, for the prediction of response to EGFR-inhibitors, KRAS status has a predictive value. This has been confirmed for metastatic colorectal cancer in a recent meta-analysis [28].

\section{Conclusion and perspective of biomarkers for treatment decisisons}

Biochemical and molecular biological factors could help in treatment decision making along the different treatment steps in the treatment of rectal cancer. This would allow more tailored treatment approaches thereby improving quality of life with even the benefit of more effective treatments. When analyzing the current value of these biomarkers in treatment decision-making, it is important to identify markers as being prognostic or predictive or even both. In rectal cancer, the only markers reaching clinical relevance in prognosis so far are deficient MMR, Kras and Braf, however its frequency in rectal cancer is rather low with only 1-4 \% deficient MMR, 30-35\% Kras mutation and 2-3\% Braf mutation [25]. The defective MMR genes hMLH-1, hMSH-2, PMS-2 and hMSH-6, detected by immunohistochemistry or by microsatellite testing showed a prognostic value in a meta-analysis [29], in the PETACC trial [30], the QUASAR and numerous single centre studies, which might lead to MMR related treatment decisions in the near future. Kras status has also been shown to be of prognostic value. Kras mutant tumors have a worse prognosis and a higher chance of recurrence. For EGFR-inhibitor therapy, the Kras status even reaches a predictive value. 
With regard to prediction, 6 genetic biomarkers were identified with the potential of being predictive in the outcome of locally advanced rectal cancer after CRT. These were p53, EGFR, TYMS, Ki-67, p21 and bax-bcl-2. While EGFR expression levels seems to provide some prediction to CRT response, p53 does not seem to serve as a good predictor, neither does Ki-67 or p21 or bax and Bcl-2. TYMS expression measurements are worthwhile to further study in future trials, while survivin showed mostly conflicting results and gene expression profiles with microarrays need further validation with larger patient groups.

Taken together, the current value of biochemical and molecular biological factors in treatment decision-making is rather low, however with many promising developments in the pipeline. Especially whole genome DNA or RNA analysis with copy number variation, multiple mutation testing, full sequencing and the association of genotype with phenotype will ultimately lead to more biomarker based treatment decisions in rectal cancer. However, successful biomarker development needs collaboration, external validation and metaanalyses to reach the level of accuracy necessary to base treatment decisions on these markers. 


\section{References}

[1] Bosset JF, Collette L, Calais G, Mineur L, Maingon P, Radosevic-Jelic L, et al. Chemotherapy with preoperative radiotherapy in rectal cancer. The New England journal of medicine. 2006;355:1114-23.

[2] Sauer R, Becker H, Hohenberger W, Rodel C, Wittekind C, Fietkau R, et al. Preoperative versus postoperative chemoradiotherapy for rectal cancer. The New England journal of medicine. 2004;351:1731-40.

[3] Swellengrebel HA, Marijnen CA, Verwaal VJ, Vincent A, Heuff G, Gerhards MF, et al. Toxicity and complications of preoperative chemoradiotherapy for locally advanced rectal cancer. The British journal of surgery. 2011;98:418-26.

[4] Marijnen CA, Nagtegaal ID, Klein Kranenbarg E, Hermans J, van de Velde CJ, Leer JW, et al. No downstaging after short-term preoperative radiotherapy in rectal cancer patients. Journal of clinical oncology : official journal of the American Society of Clinical Oncology. 2001;19:1976-84.

[5] Peeters KC, van de Velde CJ, Leer JW, Martijn H, Junggeburt JM, Kranenbarg EK, et al. Late side effects of short-course preoperative radiotherapy combined with total mesorectal excision for rectal cancer: increased bowel dysfunction in irradiated patients--a Dutch colorectal cancer group study. Journal of clinical oncology : official journal of the American Society of Clinical Oncology. 2005;23:6199-206.

[6] Stephens RJ, Thompson LC, Quirke P, Steele R, Grieve R, Couture J, et al. Impact of shortcourse preoperative radiotherapy for rectal cancer on patients' quality of life: data from the Medical Research Council CR07/National Cancer Institute of Canada Clinical Trials Group C016 randomized clinical trial. Journal of clinical oncology : official journal of the American Society of Clinical Oncology. 2010;28:4233-9.

[7] Lange MM, Maas CP, Marijnen CA, Wiggers T, Rutten HJ, Kranenbarg EK, et al. Urinary dysfunction after rectal cancer treatment is mainly caused by surgery. The British journal of surgery. 2008;95:1020-8.

[8] Pucciarelli S, Del Bianco P, Efficace F, Serpentini S, Capirci C, De Paoli A, et al. Patientreported outcomes after neoadjuvant chemoradiotherapy for rectal cancer: a multicenter prospective observational study. Annals of surgery. 2011;253:71-7.

[9] Rutten HJ, den Dulk M, Lemmens VE, van de Velde CJ, Marijnen CA. Controversies of total mesorectal excision for rectal cancer in elderly patients. The Lancet Oncology. 2008;9:494-501.

[10] Lammering G. Molecular predictor and promising target: will EGFR now become a star in radiotherapy? Radiotherapy and oncology : journal of the European Society for Therapeutic Radiology and Oncology. 2005;74:89-91.

[11] Kuremsky JG, Tepper JE, McLeod HL. Biomarkers for response to neoadjuvant chemoradiation for rectal cancer. International journal of radiation oncology, biology, physics. 2009;74:673-88. 
[12] Lamas MJ, Duran G, Gomez A, Balboa E, Anido U, Bernardez B, et al. X-ray crosscomplementing group 1 and thymidylate synthase polymorphisms might predict response to chemoradiotherapy in rectal cancer patients. International journal of radiation oncology, biology, physics. 2012;82:138-44.

[13] Paez D, Salazar J, Pare L, Pertriz L, Targarona E, del Rio E, et al. Pharmacogenetic study in rectal cancer patients treated with preoperative chemoradiotherapy: polymorphisms in thymidylate synthase, epidermal growth factor receptor, GSTP1, and DNA repair genes. International journal of radiation oncology, biology, physics. 2011;81:1319-27.

[14] Tan BR, Thomas F, Myerson RJ, Zehnbauer B, Trinkaus K, Malyapa RS, et al. Thymidylate synthase genotype-directed neoadjuvant chemoradiation for patients with rectal adenocarcinoma. Journal of clinical oncology : official journal of the American Society of Clinical Oncology. 2011;29:875-83.

[15] Grimminger PP, Brabender J, Warnecke-Eberz U, Narumiya K, Wandhofer C, Drebber U, et al. XRCC1 gene polymorphism for prediction of response and prognosis in the multimodality therapy of patients with locally advanced rectal cancer. The Journal of surgical research. 2010;164:e61-6.

[16] Kim K, Chie EK, Wu HG, Kim SG, Lee SH, Kang GH, et al. High survivin expression as a predictor of poor response to preoperative chemoradiotherapy in locally advanced rectal cancer. International journal of colorectal disease. 2011;26:1019-23.

[17] McDowell DT, Smith FM, Reynolds JV, Maher SG, Adida C, Crotty P, et al. Increased spontaneous apoptosis, but not survivin expression, is associated with histomorphologic response to neoadjuvant chemoradiation in rectal cancer. International journal of colorectal disease. 2009;24:1261-9.

[18] Rodel F, Hoffmann J, Grabenbauer GG, Papadopoulos T, Weiss C, Gunther K, et al. High survivin expression is associated with reduced apoptosis in rectal cancer and may predict disease-free survival after preoperative radiochemotherapy and surgical resection. Strahlentherapie und Onkologie : Organ der Deutschen Rontgengesellschaft [et al]. 2002;178:426-35.

[19] Kapiteijn E, Marijnen CA, Nagtegaal ID, Putter H, Steup WH, Wiggers T, et al. Preoperative radiotherapy combined with total mesorectal excision for resectable rectal cancer. The New England journal of medicine. 2001;345:638-46.

[20] Pettersson D, Cedermark B, Holm T, Radu C, Pahlman L, Glimelius B, et al. Interim analysis of the Stockholm III trial of preoperative radiotherapy regimens for rectal cancer. The British journal of surgery. 2010;97:580-7.

[21] Radu C, Berglund A, Pahlman L, Glimelius B. Short-course preoperative radiotherapy with delayed surgery in rectal cancer - a retrospective study. Radiotherapy and oncology : journal of the European Society for Therapeutic Radiology and Oncology. 2008;87:343-9. 
[22] Maas M, Beets-Tan RG, Lambregts DM, Lammering G, Nelemans PJ, Engelen SM, et al. Waitand-see policy for clinical complete responders after chemoradiation for rectal cancer. Journal of clinical oncology : official journal of the American Society of Clinical Oncology. 2011;29:4633-40.

[23] Leong KJ, Wei W, Tannahill LA, Caldwell GM, Jones CE, Morton DG, et al. Methylation profiling of rectal cancer identifies novel markers of early-stage disease. The British journal of surgery. 2011;98:724-34.

[24] Rasheed S, Harris AL, Tekkis PP, Turley H, Silver A, McDonald PJ, et al. Assessment of microvessel density and carbonic anhydrase-9 (CA-9) expression in rectal cancer. Pathology, research and practice. 2009;205:1-9.

[25] Hutchins G, Southward K, Handley K, Magill L, Beaumont C, Stahlschmidt J, et al. Value of mismatch repair, KRAS, and BRAF mutations in predicting recurrence and benefits from chemotherapy in colorectal cancer. Journal of clinical oncology : official journal of the American Society of Clinical Oncology. 2011;29:1261-70.

[26] Bujko K, Glynne-Jones R, Bujko M. Does adjuvant fluoropyrimidine-based chemotherapy provide a benefit for patients with resected rectal cancer who have already received neoadjuvant radiochemotherapy? A systematic review of randomised trials. Annals of oncology : official journal of the European Society for Medical Oncology / ESMO. 2010;21:1743-50.

[27] Valentini V, van Stiphout RG, Lammering G, Gambacorta MA, Barba MC, Bebenek M, et al. Nomograms for predicting local recurrence, distant metastases, and overall survival for patients with locally advanced rectal cancer on the basis of European randomized clinical trials. Journal of clinical oncology : official journal of the American Society of Clinical Oncology. 2011;29:3163-72.

[28] Adelstein BA, Dobbins TA, Harris CA, Marschner IC, Ward RL. A systematic review and metaanalysis of KRAS status as the determinant of response to anti-EGFR antibodies and the impact of partner chemotherapy in metastatic colorectal cancer. European journal of cancer. 2011;47:1343-54.

[29] Popat S, Hubner R, Houlston RS. Systematic review of microsatellite instability and colorectal cancer prognosis. Journal of clinical oncology : official journal of the American Society of Clinical Oncology. 2005;23:609-18.

[30] Tian S, Roepman P, Popovici V, Michaut M, Majewski I, Salazar R, et al. A robust genomic signature for the detection of colorectal cancer patients with microsatellite instability phenotype and high mutation frequency. The Journal of pathology. 2012;228:586-95. 
Chapter 6 
Part III

Combined treatment in rectal cancer

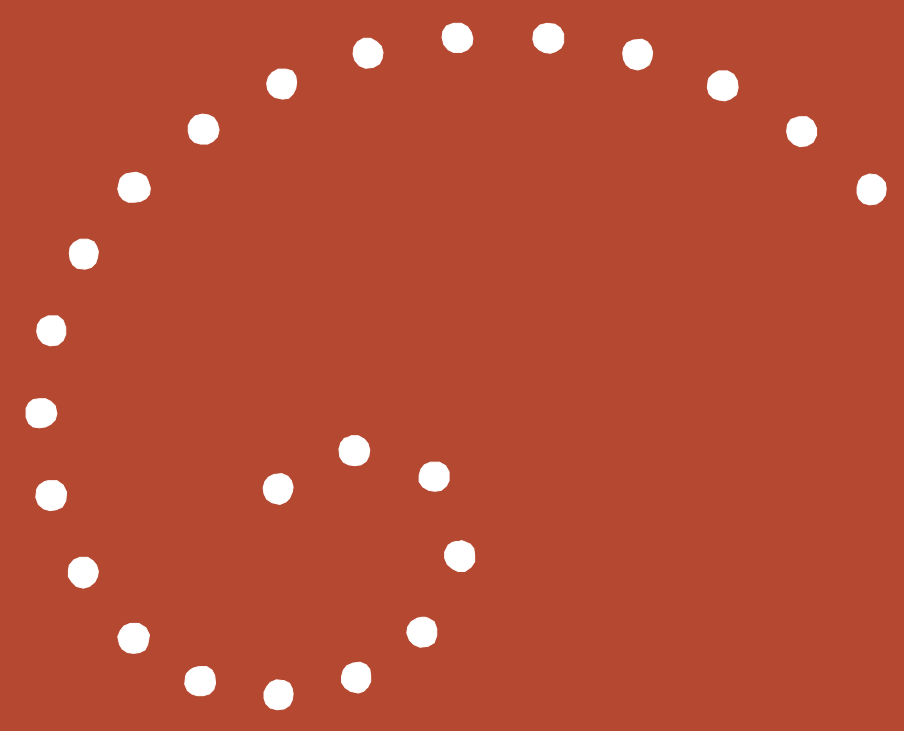





\section{Chapter 7 \\ Phase I trial of the combination of the Akt inhibitor nelfinavir and chemoradiation for locally advanced rectal cancer}

Jeroen Buijsen, Guido Lammering, Rob Jansen, Geerard Beets, Jaap Wals, Meindert Sosef, Marien Den Boer, Jeroen Leijtens, Robert Riedl, Jan Theys, Philippe Lambin 
Purpose

To investigate the toxicity of nelfinavir, administered during preoperative chemoradiotherapy (CRT) in patients with locally advanced rectal cancer.

\section{Material and methods}

Twelve patients were treated with chemoradiotherapy to 50.4 Gy combined with capecitabine $825 \mathrm{mg} / \mathrm{m}^{2}$ BID. Three dose levels (DL) of nelfinavir were tested: $750 \mathrm{mg}$ BID (DL1), $1250 \mathrm{mg}$ BID (DL2) and an intermediate level of $1000 \mathrm{mg}$ BID (DL3). Surgery was performed between 8 and 10 weeks after completion of CRT. Primary endpoint was doselimiting toxicity (DLT), defined as any grade 3 or higher non-hematological or grade 4 or higher hematological toxicity

\section{Results}

Eleven patients could be analysed: 5 were treated in DL1, 3 in DL2 and 3 in DL3. The first 3 patients in DL1 did not develop a DLT. In DL2 one patient developed gr 3 diarrhea, 1 patient had gr 3 transaminase elevation and 1 patient had a gr 3 cholangitis with unknown cause. An intermediate dose level was tested in DL3. In this group 2 patients developed gr 3 diarrhea and 1 patient gr 3 transaminase elevation and gr 4 postoperative wound complication. Three patients achieved a pathological complete response ( $p C R)$.

\section{Conclusions}

Nelfinavir 750 mg BID was defined as the recommended phase II dose in combination with capecitabine and $50.4 \mathrm{~Gy}$ pre-operative radiotherapy in rectal cancer. First tumor response evaluations are promising, but a further phase II study is needed to get more information about efficacy of this treatment regimen. 


\section{Introduction}

Pre-operative radiotherapy plays an important role in the multidisciplinary treatment of rectal cancer. It has been shown that pre-operative radiation improves local control and may increase the chance of sphincter-saving surgery $[1,2]$. In case of locally advanced rectal cancer, radiotherapy is often combined with chemotherapy to enhance the effect of radiotherapy [3]. The most frequently used combination is 5 -fluorouracil or capecitabine (an oral prodrug of 5-fluorouracil) and radiotherapy to doses around 45-50.4 Gy. This regimen results in a pathological complete response ( $P C R$ ) rate of 10-33\%, depending on radiation dose and the interval between completion of treatment and surgery [4-6]. Patients who develop a complete response have a better prognosis [7-8] and may be candidates for less invasive surgery or even a wait-and-see policy [9].

It has been shown that PI3Ks were genetically altered in 74 (32\%) of 234 human colorectal cancers [10] and that the PI3K signalling pathway can be constitutively activated in colorectal cancer [11]. Furthermore, epidermal growth factor receptor (EGFR) expression in rectal cancer is known to be an indicator of poor response to radiotherapy and a poor disease-free survival [12, 13]. Laboratory data have shown that inhibition of Ras and/or the downstream PI3K-Akt pathway increases radiosensitivity in cells in which this pathway is activated, but does not affect cells without activation of this pathway, including normal tissues $[14,15]$. As PI3K mutations seem to be common in colorectal cancer [10, 16-18], inhibition of the PI3K pathway may well be able to influence the response to radiation. In addition to PI3K mutations, this pathway may be upregulated without mutations or due to mutations of growth factor receptors (like EGFR or vascular endothelial cell growth factor receptor (VEGFR)) or downstream messengers. Furthermore, the PI3K/Akt pathway also seems to be involved in the radiosensitivity of endothelial cells. Several pre-clinical studies have demonstrated that inhibition of this pathwayincreases the radiosensitivity of tumor blood vessels even at lower fraction doses [5]. However, up until now, no clinically usable PI3K inhibitors have been available. 
HIV protease inhibitors (HPIs) are a group of drugs that have been used for more than a decade in the treatment of HIV as part of highly active anti-retroviral therapy (HAART). More recently, HPIs have been shown to possess an antitumor activity that is independent of their anti-retroviral activity and to have a radiosensitizing effect through the inhibition of phosphorylated-Akt (pAKT) [14]. The inhibition of $\mathrm{PI} 3 \mathrm{~K}$ during radiotherapy is not expected to result in increased normal tissue toxicity because this pathway is not constitutively activated in normal cells. No specific pre-clinical data is available for colorectal cancer cells,but it has been shown in vitro that inhibition of the PI3K/Akt pathway leads to radiosensitization of different cell lines in vitro and in vivo, including colon cancer, bladder cancer and glioma cell lines [19-21]. Therefore, we hypothesize that the addition of an HPI to chemoradiation for rectal cancer will increase the effect of treatment, resulting in a higher percentage of $\mathrm{pCR}$. Due to the extensive clinical experience with HPIs in HIV treatment, they form an interesting group of Akt inhibitors to test in clinical practice.The HPI nelfinavir was the most potent inhibitor of the PI3K-Akt pathway in pre-clinical studies. In HAART therapy it is prescribed in a dose of $1250 \mathrm{mg}$ BID and the most commonly observed sideeffects are diarrhea (>10\%) and transaminase elevations (around 2\%) and nausea. Since there is room for improvement in the number of pCRs and PI3K seems to be mutated in an important percentage of human colorectal cancers, we felt it was worthwhile to set up a phase I trial testing the combination of different nelfinavir dose levels with capecitabine and radiotherapy. The primary endpoint was to evaluate the toxicity of this combined modality treatment in order to find the recommended phase II dose (RP2D).

\section{Patients, Materials, and Methods}

\section{Eligibility}

Patients who were at least 18 years old and who had histologically proven adenocarcinoma of the rectum, cT3-4N0-2M0, were eligible for this study. Clinical staging was based on MRI. Additional inclusion criteria were a World Health Organization (WHO) performance status $\leq 2$ and sufficient hematological, 
renal and liver function. Concurrent medication known to be metabolized by the CYP3A4 isoenzyme was not allowed, including the use of St John's wort. Patients gave written informed consent prior to inclusion in the trial, according to the Dutch law. The study protocol was approved by the MAASTRO Institutional Review Board and the Medical Ethical Committee of Maastricht University Medical Centre. The trial was registered at ClinicalTrials.gov as NCT000704600.

\section{Treatment}

Oral nelfinavir was added to our standard chemoradiation (CRT) scheme for locally advanced rectal cancer, consisting of 28 fractions of 1.8 Gy concomitant with capecitabine $825 \mathrm{mg} / \mathrm{m}^{2}$ BID (figure 1). A 3D-conformal treatment plan was made based on a PET-CT scan. The primary tumor, complete mesorectum, presacral area, internal iliac lymph nodes and obturator region (in case of lowseated tumors) were included in the treatment volume [22]. Three-dimensional treatment planning was used to obtain adequate coverage and a homogeneous dose distribution.

Two dose levels (DLs 1 and 2) of nelfinavir were planned to test with the possibility of exploring an intermediate DL3 if DL2 turned out to be too toxic. Dose levels were defined as $750 \mathrm{mg}$ nelfinavir BID for DL1, $1250 \mathrm{mg}$ BID for DL2 and $1000 \mathrm{mg}$ BID for DL3. Nelfinavir was started seven days before the start of chemoradiotherapy and nelfinavir plasma levels were monitored in weeks two, four and six, using an HPLC-UV method. To correct for the different timepoints at which samples for drug level measurement were taken, the drug concentration ratio (CR) was calculated for each sample [23] by dividing the drug concentration measured in that sample by the time-adjusted value in the standardized pharmacokinetic curve.

Surgical resection (either a low anterior resection or abdominoperineal resection, left to the discretion of the surgeon) was performed preferably between eight to ten weeks after the end of CRT.

Patients who had node-positive disease, either on MRI or pathologic examination, received adjuvant chemotherapy (capacitabin/oxaliplatin). According to the protocol of the referring hospital and the condition of the 
patient, 1 or 2 cycles could be administered in the interval between the completion of CRT and resection.

\section{Toxicity evaluation}

The National Cancer Institute's Common Terminology Criteria for Adverse Events version 3.0 ( $\mathrm{NCl}-\mathrm{CTCAE}$ ) was used to score acute toxic effects of chemoradiotherapy and post-operative toxicity [24]. Dose limiting toxicity was defined as any grade 3 or more non-hematologic toxicity, any grade 4 or more hematologic toxicity or any grade 4 or higher post-operative toxicity within 30 days post-surgery.

Toxicity was scored weekly during chemoradiation and one, two and four weeks after the end of CRT. Post-operative toxicity was scored weekly until four weeks after surgery. Nelfinavir doses were adjusted if CRs were lower than 0.90 or higher than 1.5 .

Three patients were initially given DL1 (750 mg BID). If none of the three patients experienced a dose-limiting toxicity (DLT), the second dose level was investigated. If one-third of the patients had grade 4 or two-thirds had grade 3 toxicity, up to three additional patients were added; if none of the additional patients developed DLT, the second dose level was investigated. Otherwise the RP2D was exceeded in the first dose level.

Three patients were initially given DL2 (1250 mg BID). If one-third experienced DLT, then up to three additional patients were entered. If no more than one of the five patients developed DLT, then the second dose level was declared the RP2D. Otherwise, the RP2D was exceeded and the first dose level would be expanded to a total of five patients (if only three patients had been treated). Thereafter, an intermediate dose level of $1000 \mathrm{mg}$ BID could be explored. If at least two of the three patients had DLT, the RP2D was exceeded and the first dose level would be declared the RP2D.

\section{Response evaluation}

All patients underwent a resection. Resection specimens were analyzed in a standardized way according to the national guidelines. Tumor regression grade 
(TRG) was scored by one pathologist according to the Mandard scoring system [25]. In short, scores ranged from TRG5 (no changes) to TRG1 (no viable tumor cells left).

\section{Statistical methods}

The primary objective of this study was to evaluate the safety of nelfinavir, capecitabine and radiotherapy and to identify the RP2D of nelfinavir among patients with locally advanced rectal cancer. A standard 3+3 dose escalation design (modified Fibonacci design) was used [26]. Categorical variables were summarized using frequencies, whereas continuous variables were reported using the median (range).

\section{Results}

\section{Patient characteristics}

Twelve patients (five DL1, three DL2 and four DL3) were enrolled in the study between October 2008 and September 2010. One patient in DL3 had no measurable nelfinavir levels at three different time points. Although he affirmed that he took the medication as prescribed, we decided to exclude this patient from further analysis and include an extra patient in DL2 since we judged this patient to be unrepresentative for toxicity analysis. Therefore only 11 patients were analyzed. Patient characteristics are shown in table 1.

\section{Toxicities}

The first three DL1 patients did not experience any DLT. In DL2, one patient developed a grade 3 transaminase elevation (without clinical signs), defined as more than five times the upper limit of normal, and one patient had a grade 3 cholangitis for which no specific cause could be found. Therefore, a relationship with the treatment regimen could not be ruled out. In both patients, symptoms disappeared and lab values normalized after nelfinavir was stopped. Radiotherapy treatment could be completed and both events were graded as DLT. The third patient had grade 2 diarrhea and developed a grade 3 ileus 
caused by excessive use of loperamide. This grade 3 toxicity was considered to be at most indirectly related to the use of nelfinavir. Radiotherapy had to be interrupted and stopped after $32.4 \mathrm{~Gy}$ in this case.

Table 1 Patient characteristics

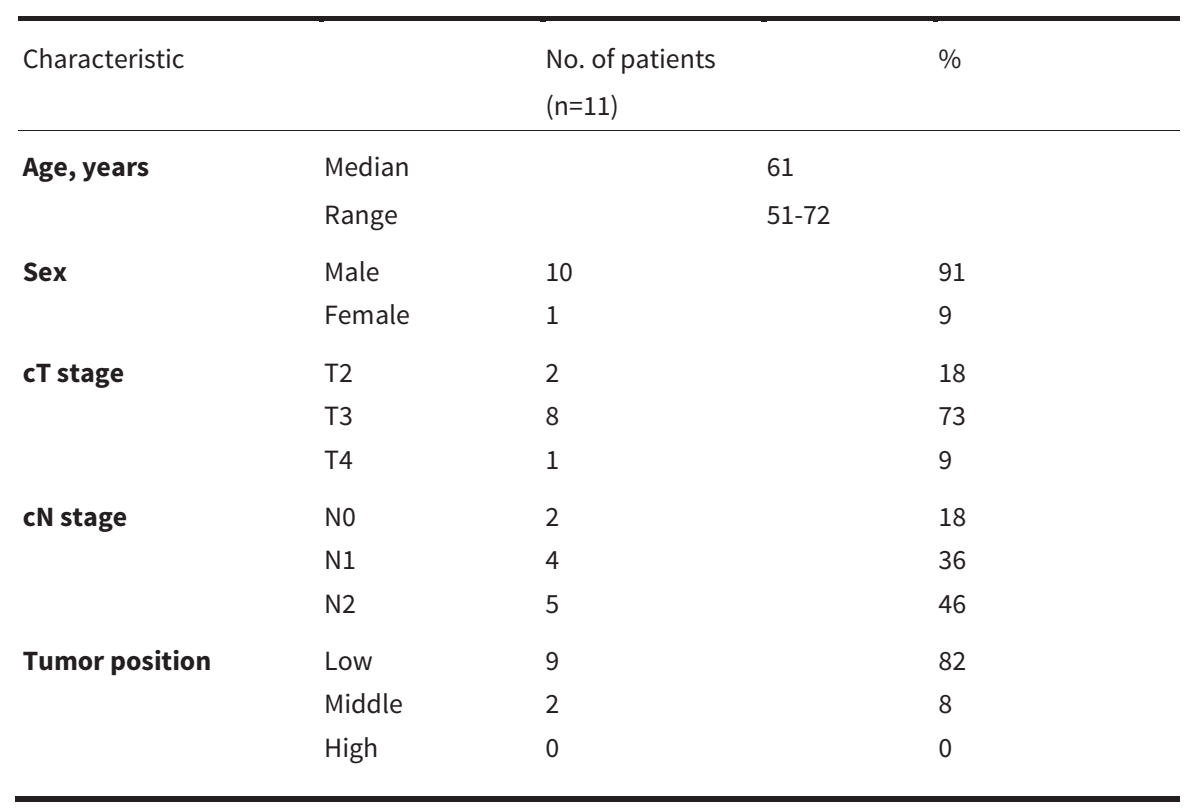

Because $2 / 3$ patients developed DLT, DL2 was deemed too toxic and according to the definitions of the protocol DL1 was expanded with another two patients, who did not experience any toxicity $\geq$ grade 3 . Therefore an intermediate nelfinavir dose was explored in DL3. At this dose level two of the three patients were hospitalized for grade 3 diarrhea. Radiotherapy could be completed in both patients. The third patient showed a grade 3 transaminase elevation, again without clinical symptoms, which normalized after stopping nelfinavir and he developed a grade 4 wound complication of the abdominal wound for which surgical revision was necessary. Although a relationship between this wound complication and the study medication seems to be unlikely, it cannot be ruled out completely. Because DLT occurred in all three patients in DL3, DL1 was declared the MTD. An overview of all toxicities $\geq$ grade 3 is shown in table 2. As can 
be concluded from the overall treatment times (OTT) mentioned in this table, a treatment interruption of more than five days was necessary for one patient.

Table 2 Acute toxicity during chemoradiation until 4 weeks post-surgery

\begin{tabular}{|c|c|c|c|c|c|}
\hline $\begin{array}{l}\text { Patient } \\
\text { no }\end{array}$ & Dose level NFV & $\begin{array}{l}\text { Toxicity } \\
\geqq \text { gr } 3\end{array}$ & $\begin{array}{l}\text { Dose } \\
\text { adjustment } \\
\text { NFV }^{2}\end{array}$ & Description & $\begin{array}{l}\text { Relation } \\
\text { with study } \\
\text { medication }\end{array}$ \\
\hline 1 & 750 mg BID & $\mathrm{n}$ & $\mathrm{n}$ & & \\
\hline 2 & 750 mg BID & $\mathrm{n}$ & $\mathrm{n}$ & & \\
\hline 3 & 750 mg BID & $\mathrm{n}$ & $\mathrm{n}$ & & \\
\hline 7 & 750 mg BID & $\mathrm{n}$ & $\mathrm{n}$ & & \\
\hline 8 & 750 mg BID & $\mathrm{n}$ & $\mathrm{n}$ & & \\
\hline 9 & 1000 mg BID & y & $y$ & $\begin{array}{l}\text { grade } 3 \text { transaminase elevation } \\
\text { grade } 4 \text { wound dehiscence }\end{array}$ & $\begin{array}{l}\text { likely } \\
\text { unlikely }\end{array}$ \\
\hline 10 & $1000 \mathrm{mg}$ BID & $\mathrm{y}$ & $\mathrm{y}$ & grade 3 diarrhea & possible \\
\hline 11 & 1000 mg BID & y & y & grade 3 diarrhea & possible \\
\hline 4 & $1250 \mathrm{mg}$ BID & y & $n$ & grade 3 ileus & indirectly \\
\hline 5 & $1250 \mathrm{mg}$ BID & y & $\mathrm{n}$ & grade 3 transaminase elevation & likely \\
\hline 6 & $1250 \mathrm{mg}$ BID & $\mathrm{y}$ & $\mathrm{y}$ & grade 3 cholangitis & possible \\
\hline
\end{tabular}

${ }^{1}$ In chronological order of inclusion in the trial.

${ }^{2}$ Dose adjustment due to high plasma levels.

\section{Nelfinavir plasma levels}

Nelfinavir plasma levels turned out to be quite variable between patients. In our patient group we observed mainly high CRs: the median CRs were 1.7 (range 1.2-7.1), 1.2 (0.6-6.0) and 1.7 (1.1-4.1) respectively in weeks two, four and six of nelfinavir use. In four patients (three in DL2 and one in DL3), the prescribed nelfinavir dose was adapted because of $\mathrm{CRs}>1.5$. All patients who needed a dose adjustment also developed a DLT. In total $4 / 6$ patients with DLT also had a $C R>1.5$. No clear relationship between the height of $C R$ and the occurrence of DLT was observed. 
Table 3 Treatment details and response to therapy

\begin{tabular}{|c|c|c|c|c|c|c|c|c|c|c|}
\hline $\begin{array}{l}\text { Patient } \\
\text { no }\end{array}$ & $\begin{array}{l}\text { Dose level } \\
\text { NFV }\end{array}$ & $\begin{array}{l}\text { NFV } \\
\text { com- } \\
\text { pleted }\end{array}$ & $\begin{array}{l}\text { RT } \\
\text { dose } \\
\text { (Gy) }\end{array}$ & $\begin{array}{l}\text { OTT } \\
\text { (days) }\end{array}$ & $\begin{array}{l}\text { Adj } \\
\text { chemo }^{1}\end{array}$ & $\begin{array}{l}\text { Interval } \\
\text { RT } \\
\text { surgery } \\
\text { (days) }\end{array}$ & $\begin{array}{l}\text { Type } \\
\text { of } \\
\text { surgery }\end{array}$ & $\begin{array}{l}\text { CTN } \\
\text { stage }\end{array}$ & $\begin{array}{l}\text { ypTN } \\
\text { stage }\end{array}$ & $\mathrm{TRG}^{2}$ \\
\hline 1 & $750 \mathrm{mg}$ BID & $\mathrm{y}$ & 50.4 & 37 & $\mathrm{n}$ & 58 & APR & cT3N2 & ypT1N0 & 2 \\
\hline 2 & 750 mg BID & $\mathrm{y}$ & 50.4 & 37 & $y, 2$ & 76 & LAR & cT3N2 & ypT3N0 & 3 \\
\hline 3 & $750 \mathrm{mg}$ BID & $\mathrm{y}$ & 50.4 & 37 & $y, 1$ & 58 & APR & $\mathrm{cT} 2 \mathrm{~N} 2$ & урт3N2 & 4 \\
\hline 7 & 750 mg BID & $y$ & 50.4 & 39 & $\mathrm{n}$ & 49 & APR & CT3NO & урT3N0 & 3 \\
\hline 8 & 750 mg BID & $\mathrm{y}$ & 50.4 & 38 & $\mathrm{n}$ & 59 & APR & cT3N1 & ypT3N0 & 3 \\
\hline 9 & $1000 \mathrm{mg}$ BID & $\mathrm{y}$ & 50.4 & 38 & $\mathrm{n}$ & 58 & APR & cT3N1 & ypTONO & 1 \\
\hline 10 & 1000 mg BID & $n$ & 50.4 & 37 & $y, 2$ & 71 & LAR & cT4N2 & урTONO & 1 \\
\hline 11 & 1000 mg BID & $\mathrm{n}$ & 50.4 & 53 & $\mathrm{n}$ & 63 & APR & cT2N1 & урт2N0 & 4 \\
\hline 4 & $1250 \mathrm{mg}$ BID & $\mathrm{n}$ & 32.4 & 23 & $\mathrm{n}$ & 112 & LAR & cT3N2 & ypT3N0 & 5 \\
\hline 5 & 1250 mg BID & $\mathrm{n}$ & 50.4 & 37 & $\mathrm{n}$ & 63 & LAR & cT3N1 & ypTONO & 1 \\
\hline 6 & $1250 \mathrm{mg}$ BID & $n$ & 50.4 & 40 & $\mathrm{n}$ & 72 & LAR & cT3NO & ypT2N0 & 3 \\
\hline
\end{tabular}

${ }^{1}$ Adjuvant chemotherapy given before surgery and if yes number of cycles.

${ }^{2}$ TRG scores according to Mandard.

Resections, response and follow-up

All patients underwent a radical resection: an abdominoperineal resection in six cases and a low anterior resection in five cases. The mean interval between radiotherapy and surgery was 67 days (range: 49-112 days). Table 3 shows the responses of all patients. Three patients $(27 \%)$ showed a pathological complete response. These pCRs were all observed in DL2 and 3. Taking TRG into account and considering TRG1 and 2 to be good responders, four patients had a good response (TRG1 and 2). T-downstaging was found in five (45\%) patients and $\mathrm{N}$ downstaging in eight $(73 \%)$ patients. Surgery resulted in a radical resection in all patients. At the time of analysis, all patients were alive without local or distant recurrence with a follow-up between five and 27 months. 


\section{Discussion}

This study demonstrates that the combination of capecitabine, radiotherapy and nelfinavir is feasible, although the RP2D in this combination turned out to be the lowest dose level tested (750 mg BID). This is the third phase I study evaluating the use of a protease inhibitor in combination with chemotherapy and radiotherapy and the first study in rectal cancer patients. The other studies evaluated pancreatic cancer and non-small cell lung cancer patients [27, 28]. In these studies, nelfinavir was added to radiotherapy combined with gemcitabine and cisplatin the pancreas trial and radiotherapy and cisplatinum and etoposide in the lung trial. In contrast to our observations, no DLT related to the study medication was observed in both studies. The reason for that may be the different chemotherapy used in both studies and the different toxicity profiles of radiation of the upper abdomen versus the pelvic region.

In this study design we chose to escalate the dose of nelfinavir because the combination of radiotherapy and capecitabine in a dose of $825 \mathrm{mg} / \mathrm{m}^{2}$ is already well established and widely used. Diarrhea is a well-knownside effect of this treatment regimen. In a recent series of 147 patients treated with $25 \times 2$ Gy in combination with capecitabine, severe diarrhea (grade 3) was observed in $10.2 \%$ of patients [29]. Furthermore, diarrhea has been reported in up to $50 \%$ of patients treated with capecitabine monotherapy, of which $15 \%$ was classified as severe diarrhea ( $13 \%$ grade $3,2 \%$ grade 4 ) [30]. Because diarrhea is also a frequently occurring side effect of nelfinavir [31], we decided to start with a relatively low dose (750 mg BID) of this drug. In pre-clinical studies, nelfinavir has been tested at concentrations normally achieved in HIV positive patients treated with HAART and it has been shown that higher doses only slightly increased radiation response. Therefore, the dose used in HIV treatment (1250 $\mathrm{mg}$ BID) was used as the highest dose level.

Diarrhea was observed frequently in this study: three patients developed grade 3 diarrhea for which they were hospitalized. Severe diarrhea ( $\geq$ grade 3 ) was only observed in DL2 and DL3. Patients who have a deficiency in dihydropyrimidine dehydrogenase (DPD), an important enzyme in the catabolism of 5-FU of which 
capecitabine is a prodrug, can develop severe toxicity during capecitabine use. This may have influenced the endpoint of this study. Although patients in this study were not tested for DPD deficiency, we had no clinical suspicion of such a deficiency as these patients often develop severe toxicity of multiple organ systems, including a very pronounced mucositis, early during treatment.

It was quite remarkable that two patients showed grade 3 transaminase elevations and one patient developed a grade 3 cholangitis. All patients in this study had normal liver function tests before start of treatment. In trials testing nelfinavir for treatment of HIV, the reported incidence of transaminase elevations was around $2 \%$, thus it is not a frequently occurring side effect [32]. Although elevated liver enzymes are reported frequently (1-10\%) during capecitabine monotherapy according to the product information, this side effect is often mild and seldom a reason for dose adjustments [30].

Hepatotoxicity has also been described with the use of HPIs, though the reported rate of severe hepatotoxicity differs between different HPIs. None of the patients in this study had a pre-existing liver disease. In a meta-analysis including four studies looking at hepatotoxicity, nelfinavir showed the lowest rate of transaminase elevations as compared to other HPIs [32]. No additive effect of liver toxicity between capecitabine and nelfinavir was described in the literature. It is not clear whether the case of cholangitis was treatment related, but no other clear cause could be found. Therefore a relationship with the study drug could not be ruled out.

Four out of six patients who developed DLT also needed a dose adjustment of NFV because high plasma levels. Although the most important P450 isoenzymes in the metabolism of nelfinavir are CYP3A and CYP2C19, CYP2C9 is also involved in the metabolism of nelfinavir. Capecitabine is an inhibitor of CYP2C9. This could be a possible explanation of the relative high number of patients showing high nelfinavir plasma levels requiring dose adjustments. In an important part of the Caucasian population polymorphisms of CYP2C19 and CYP2C9 have been described [33]. These polymorphisms can also lead to a different metabolism. Another possible explanation could be a different absorption of nelfinavir due to mucosa changes in the gastrointestinal tract caused by nelfinavir. From the 
experience in HIV treatment no connection between high plasma levels and toxicity has been described. Although our observationsindicate a possible relationship between high NFV plasma levels and toxicity, the number of patients in this phase I trial is too small to draw conclusions.

Three of 11 patients (27\%) had a pathological complete response and four of 11 patients (36\%) had a major response taking TRG into account. Although the percentage is rather high compared to response rates reported in trials using a comparable chemoradiation regimen [7], no conclusions can be drawn about the additive effect of nelfinavir because of the small sample. All three complete responders were treated in dose level 2 or 3 , but this could be by chance.

The radiosensitizing effects of HPIs have been attributed to several mechanisms. The first mechanism is the inhibition of Akt phosphorylation [14]. In pre-clinical studies, drug concentrations comparable with therapeutic levels achieved in HIV patients were shown to inhibit Akt. Apart from dephosphorylation of Akt, HPIs are thought to have direct anti-tumor effects through several other mechanisms. Amongst the mechanisms described in literature are proteasome inhibition, the inhibition of matrix metalloproteinases and immunomodulatory effects [34], as well as stimulation of apoptosis and autophagy [35] and improvement of vascular flow and decrease of hypoxia [36, 37]. In combination with radiation, HPIs have effects on endothelial cells, leading to increased apoptosis of endothelial cells and blockage of endothelial cell migration and organization [38]. It is important to note that these mechanisms occur at different nelfinavir concentrations in laboratory studies.

One of the downsides of nelfinavir is that plasma levels can vary importantly between patients and that there is an interaction with a broad group of drugs [39]. In the treatment of HIV patients, concentration ratios $<0.90$ are frequently observed [23]. In our patient group, we mainly observed CRs that were higher than expected: the dose adjustments were all results of too-high plasma levels. Three of the four patients needed a dose adjustment developed DLT, but it is not known whether there is a relationship between plasma levels and the chance of toxicity. For diarrhea, no clear relationship has been shown between dose levels and the chance of severe diarrhea [40] and no information is 
available about dose levels and the chance of hepatotoxicity.

In conclusion, this study shows that the combination of nelfinavir with capecitabine-based chemoradiation in locally advanced rectal cancer is feasible, but the toxicity rates are rather high. From this phase I trial, nelfinavir $750 \mathrm{mg} \mathrm{BID}$ turned out to be the RP2D. A further phase II study is needed to learn more about the safety and efficacy of this combination treatment. 


\section{References}

[1] Cunningham, D, Atkin, W, Lenz, HJ, et al. Colorectal cancer. Lancet 2010;375:1030-1047.

[2] Mohiuddin, M, Marks, J, Marks, G. Management of rectal cancer: short- vs. long-course preoperative radiation. Int J Radiat Oncol Biol Phys 2008;72:636-643.

[3] Valentini, V, Aristei, C, Glimelius, B, et al. Multidisciplinary Rectal Cancer Management: 2nd European Rectal Cancer Consensus Conference (EURECA-CC2). Radiother Oncol 2009;92:148-163.

[4] Balch, GC, De Meo, A, Guillem, JG. Modern management of rectal cancer: a 2006 update. World J Gastroenterol 2006;12:3186-3195.

[5] Kim, DW, Huamani, J, Fu, A, Hallahan, DE. Molecular strategies targeting the host component of cancer to enhance tumor response to radiation therapy. Int $\mathrm{J}$ Radiat Oncol Biol Phys 2006;64:38-46.

[6] Wiltshire, KL, Ward, IG, Swallow, C, et al. Preoperative radiation with concurrent chemotherapy for resectable rectal cancer: effect of dose escalation on pathologic complete response, local recurrence-free survival, disease-free survival, and overall survival. Int J Radiat Oncol Biol Phys 2006;64:709-716.

[7] Maas, M, Nelemans, PJ, Valentini, V, et al. Long-term outcome in patients with a pathological complete response after chemoradiation for rectal cancer: a pooled analysis of individual patient data. Lancet Oncol 2010;11:835-844.

[8] Valentini, V, Lambin, P, Myerson, RJ. Is it time for tailored treatment of rectal cancer? From prescribing by consensus to prescribing by numbers. Radiother Oncol 2012;102:1-3.

[9] Maas, M, Beets-Tan, RG, Lambregts, DM, et al. Wait-and-See Policy for Clinical Complete Responders After Chemoradiation for Rectal Cancer. J Clin Oncol 2011.

[10] Samuels, Y, Wang, Z, Bardelli, A, et al. High frequency of mutations of the PIK3CA gene in human cancers. Science 2004;304:554.

[11] Vivanco, I, Sawyers, CL. The phosphatidylinositol 3-Kinase AKT pathway in human cancer. Nat Rev Cancer 2002;2:489-501.

[12] Giralt, J, de las Heras, M, Cerezo, L, et al. The expression of epidermal growth factor receptor results in a worse prognosis for patients with rectal cancer treated with preoperative radiotherapy: a multicenter, retrospective analysis. Radiother Oncol 2005;74:101-108.

[13] Kuremsky, JG, Tepper, JE, McLeod, HL. Biomarkers for response to neoadjuvant chemoradiation for rectal cancer. Int J Radiat Oncol Biol Phys 2009;74:673-688.

[14] Gupta, AK, Cerniglia, GJ, Mick, R, McKenna, WG, Muschel, RJ. HIV protease inhibitors block Akt signaling and radiosensitize tumor cells both in vitro and in vivo. Cancer Res 2005;65:82568265.

[15] Gupta, AK, McKenna, WG, Weber, CN, et al. Local recurrence in head and neck cancer: relationship to radiation resistance and signal transduction. Clin Cancer Res 2002;8:885-892. 
[16] Barault, L, Veyrie, N, Jooste, V, et al. Mutations in the RAS-MAPK, PI(3)K (phosphatidylinositol3-OH kinase) signaling network correlate with poor survival in a population-based series of colon cancers. Int J Cancer 2008;122:2255-2259.

[17] He, Y, Van't Veer, LJ, Mikolajewska-Hanclich, I, et al. PIK3CA mutations predict local recurrences in rectal cancer patients. Clin Cancer Res 2009;15:6956-6962.

[18] Liao, X, Morikawa, T, Lochhead, P, et al. Prognostic role of PIK3CA mutation in colorectal cancer: cohort study and literature review. Clin Cancer Res 2012;18:2257-2268.

[19] Gupta, AK, Cerniglia, GJ, Mick, R, et al. Radiation sensitization of human cancer cells in vivo by inhibiting the activity of PI3K using LY294002. Int J Radiat Oncol Biol Phys 2003;56:846-853.

[20] Gupta, AK, Bakanauskas, VJ, Cerniglia, GJ, et al. The Ras radiation resistance pathway. Cancer Res 2001;61:4278-4282.

[21] Jiang, W, Mikochik, PJ, Ra, JH, et al. HIV protease inhibitor nelfinavir inhibits growth of human melanoma cells by induction of cell cycle arrest. Cancer Res 2007;67:1221-1227.

[22] Nijkamp, J, de Haas-Kock, DF, Beukema, JC, et al. Target volume delineation variation in radiotherapy for early stage rectal cancer in the Netherlands. Radiother Oncol 2012;102:14-21.

[23] Burger, D, Hugen, P, Reiss, $P$, et al. Therapeutic drug monitoring of nelfinavir and indinavir in treatment-naive HIV-1-infected individuals. AIDS 2003;17:1157-1165.

[24] http://ctep.cancer.gov/protocolDevelopment/electronic_applications/docs/ctcaev3.pdf

[25] Mandard, AM, Dalibard, F, Mandard, JC, et al. Pathologic assessment of tumor regression after preoperative chemoradiotherapy of esophageal carcinoma. Clinicopathologic correlations. Cancer 1994;73:2680-2686.

[26] Pijls-Johannesma, M, van Mastrigt, G, Hahn, SM, et al. A systematic methodology review of phase I radiation dose escalation trials. Radiother Oncol 2010;95:135-141.

[27] Brunner, TB, Geiger, M, Grabenbauer, GG, et al. Phase I trial of the human immunodeficiency virus protease inhibitor nelfinavir and chemoradiation for locally advanced pancreatic cancer. J Clin Oncol 2008;26:2699-2706.

[28] Rengan, R, Mick, R, Pryma, D, et al. A phase I trial of the HIV protease inhibitor nelfinavir with concurrent chemoradiotherapy for unresectable stage IIIA/IIIB non-small cell lung cancer: a report of toxicities and clinical response. J Thorac Oncol 2012;7:709-715.

[29] Swellengrebel, HA, Marijnen, CA, Verwaal, VJ, et al. Toxicity and complications of preoperative chemoradiotherapy for locally advanced rectal cancer. Br J Surg 2011;98:418-426.

[30] Walko, CM, Lindley, C. Capecitabine: a review. Clin Ther 2005;27:23-44.

[31] Viracept ${ }^{\oplus}$. Prescribing Information. APILJ, CA 92037, USA Item \#634200MV. 2001.

[32] Bruno, R, Sacchi, P, Maiocchi, L, Zocchetti, C, Filice, G. Hepatotoxicity and nelfinavir: a metaanalysis. Clin Gastroenterol Hepatol 2005;3:482-488.

[33] Kurose, K, Sugiyama, E, Saito, Y. Population differences in major functional polymorphisms of pharmacokinetics/pharmacodynamics-related genes in Eastern Asians and Europeans: 
implications in the clinical trials for novel drug development. Drug Metab Pharmacokinet 2012;27:9-54.

[34] Monini, P, Sgadari, C, Toschi, E, Barillari, G, Ensoli, B. Antitumor effects of antiretroviral therapy. Nat Rev Cancer 2004;4:861-875.

[35] Gills, JJ, Lopiccolo, J, Tsurutani, J, et al. Nelfinavir, A lead HIV protease inhibitor, is a broadspectrum, anticancer agent that induces endoplasmic reticulum stress, autophagy, and apoptosis in vitro and in vivo. Clin Cancer Res 2007;13:5183-5194.

[36] Qayum, N, Muschel, RJ, Im, JH, et al. Tumor vascular changes mediated by inhibition of oncogenic signaling. Cancer Res 2009;69:6347-6354.

[37] Fokas, E, Im, JH, Hill, S, et al. Dual inhibition of the PI3K/mTOR pathway increases tumor radiosensitivity by normalizing tumor vasculature. Cancer Res 2012;72:239-248.

[38] Cuneo, KC, Tu, T, Geng, L, Fu, A, Hallahan, DE, Willey, CD. HIV protease inhibitors enhance the efficacy of irradiation. Cancer Res 2007;67:4886-4893.

[39] Perry, CM, Frampton, JE, McCormack, PL, Siddiqui, MA, Cvetkovic, RS. Nelfinavir: a review of its use in the management of HIV infection. Drugs 2005;65:2209-2244.

[40] Droste, JA, Koopmans, PP, Hekster, YA, Burger, DM. TDM: therapeutic drug measuring or therapeutic drug monitoring? Ther Drug Monit 2005;27:412-416. 


\section{Chapter 8 \\ A phase I-II study on the combination of rapamycin and short course radiotherapy in rectal cancer}

Jeroen Buijsen, Jørgen van den Bogaard, Barry Jutten, Eric Belgers, Meindert Sosef, Jeroen W.A. Leijtens, Geerard L. Beets, Rob L.H. Jansen, Robert G. Riedl, Ruud Clarijs, Guido Lammering ${ }^{*}$, Philippe Lambin*

*Equal contribution 


\section{Purpose}

This phase I/II study sought to determine the safety and maximum tolerated dose (MTD) of the combination of rapamycin, an mTOR inhibitor, with short-course radiotherapy in rectal cancer patients. Antitumor activity, changes in metabolic activity and perfusion on imaging, and changes in phosphorylation status of the mTOR pathway were also assessed.

\section{Experimental design}

Patients with primary resectable rectal cancer were treated with short-course hypofractionated radiotherapy (5x5 Gy), followed by surgical resection.

\section{Results}

Thirteen patients were entered in phase I. One patient developed a dose-limiting toxicity, consisting of a grade 4 leak and grade 4 bleeding. Because of an unexpected high rate of grade 3 postoperative toxicity, it was decided to treat patients with delayed surgery in phase II. Thirty-one patients were treated with the MTD of $6 \mathrm{mg}$ rapamycin daily. One patient (3\%) developed a pathological complete response (pCR) and 3 patients (10\%) had a ypT1NO tumor at the time of resection. No change in tumor perfusion was observed on perfusion $\mathrm{CT}$, but a significant decrease of metabolic activity was found on PET-scan.

\section{Conclusion}

The combination of short-course radiotherapy and rapamycin turned out to be feasible, provided that the interval between neo-adjuvant treatment and surgical resection is at least 6 weeks. Although from this cohort no clear increase in PCR could be observed, a clear metabolic response after rapamycin run-in was observed, indicating a biological activity of this drug in rectal cancer. 


\section{Introduction}

Pre-operative radiotherapy has an important role in the treatment of rectal cancer. For primary resectable rectal cancer, short-course hypofractionated radiotherapy $(5 \times 5 \mathrm{~Gy})$ results in a $50 \%$ reduction of local recurrence [1]. If resection takes place within a week after the completion of radiotherapy, no downsizing of the tumor will occur. However, after an interval of at least 6 week, substantial downsizing can be observed $[2,3]$. As this short course schedule is primarily used for relatively small tumors, a part of the patients will develop a complete response. This opens the way for alternative organ preservation treatment strategies [4]. In view of the relatively limited toxicity observed with this treatment, there is room for treatment intensification.

In order to further increase the tumor response rates, radiotherapy can be combined with radiosensitizing drugs. The mammalian target of rapamycin (mTOR) pathway is attractive to exploit, because it plays a central role in survival strategies of tumor cells and influences angiogenesis [5]. Furthermore, it has been shown that mTOR has a direct link with the phosphatidylinositol-3'kinase (PI3K)/PTEN-AKT survival pathway [6]. Preclinical evidence shows a radiosensitizing effect of $\mathrm{mTOR}$ inhibitors $[7,8]$. Especially the link between mTOR inhibition and the inhibition of angiogenesis may be important for the radiosensitizing effects [9]. Neovasculature in tumors tends to be unstructured and leaky, leading to an inefficient blood flow and hypoxic areas. Antiangiogenic treatments may result in normalization of this vasculature resulting in improved oxygen supply [10].

Rapamycin is the oldest mTOR inhibitor used for a long time as an immune suppressant. It was shown more than 20 years ago that rapamycin also had antiproliferative effects in tumor cell lines. During the last years analogues of rapamycin have been developed as anti-cancer drugs. The advantage of rapamycin is that there is extensive clinical experience with its use as immune suppressant after kidney transplantation and it is an oral drug.

The objective of this trial was to investigate the safety and the activity of rapamycin, administered before and during preoperative radiotherapy in 
patients with resectable rectal cancer. The purpose of phase I was to determine the recommended phase II dose (RP2D). For phase II of this study, change in tumor perfusion was chosen as the primary endpoint. We believe this is an attractive surrogate endpoint predicting response, as it can be measured early after the start of treatment. Furthermore it gives insight in the mechanism of action of rapamycin in rectal cancer with and without radiation. It was hypothesized that rapamycin would lead to a decrease of the activation status of mTOR related and dependent molecules in the tumor reflected by a decrease in staining for phosphorylated mTOR (p-mTOR) and phosphorylated 4E-BP1in tumor biopsies. 4E-BP1 is a protein that is activated and dissociated from elF4E after phosphorylation, leading to increased translation, and is downstream effector of mTOR complex 1 (mTORC1).

\section{Methods and materials}

This was an open-label, single-institution prospective phase I/II trial, approved by the local Medical Ethical Committee. Radiotherapy was performed at Maastro Clinic, surgery was done in the referring hospital. All patients gave written informed consent. The trial has been registered at clinicaltrials.cov (NCT00409994).

\section{Patient eligibility and treatment}

Patients with a primary resectable rectal cancer, defined as CT2-3 without involvement of the circumferential resection margin (CRM) as judged on MRI and $\mathrm{cNO}$-1, for whom short course pre-operative radiotherapy was advised in the Tumor Board meeting, were eligible for inclusion in the trial. The most important exclusion criterion was the concurrent use of CYP3A4 inhibiting drugs.

An outline of the study is presented in figure 1. Radiotherapy treatment consisted of 5 fractions of 5 Gy given within an overall treatment time of 7 days. The treated volume consisted of the primary tumor, mesorectum, presacral area and internal iliac lymph nodes. Patients were treated using a 4-field 3D- 
conformal technique or a VMAT technique. During phase I patients were operated within 3 days after the last fraction of radiotherapy. In phase II resection was performed preferably 7-8 weeks after completion of radiotherapy, but a minimum interval of 6 weeks was required.
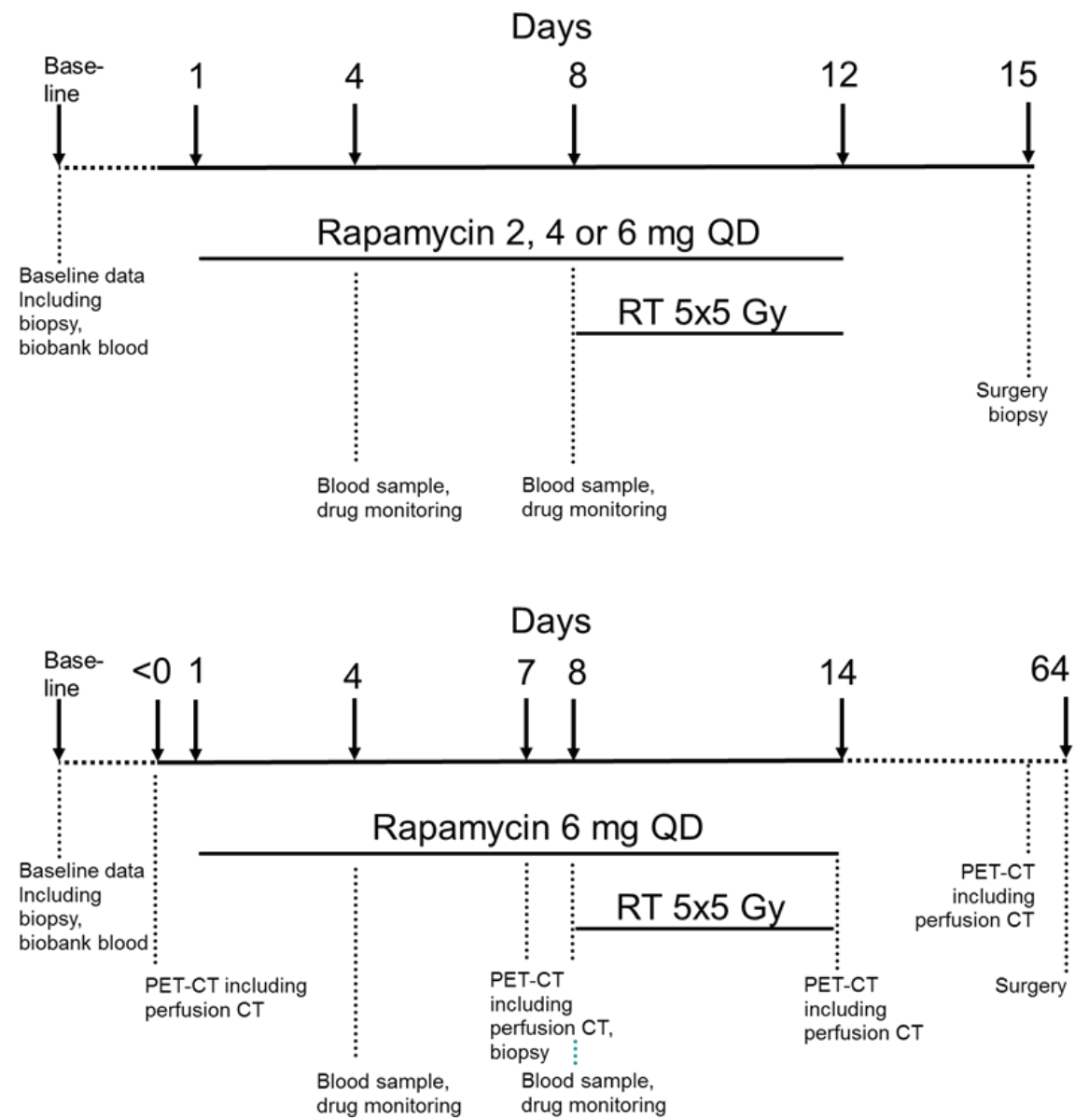

Figure 1 Outline of phase I (upper panel) and phase II (lower panel) of the study.

Rapamycin treatment was started 1 week before the start of radiotherapy. For phase I, three dose levels were defined: $2 \mathrm{mg}, 4 \mathrm{mg}$ and $6 \mathrm{mg}$ daily respectively. Cohorts of 3-6 patients were treated. If no DLT occurred, then subsequent 
patients were treated at the next dose level. In order to monitor rapamycin plasma levels, blood samples were taken on day 4, 8 and 15. Trough levels aimed at were 5-15 $\mu \mathrm{g} / \mathrm{l}$ for dose level (DL) 1, 10-30 $\mu \mathrm{g} / \mathrm{l}$ for DL2 and 15-45 $\mu \mathrm{g} / \mathrm{l}$ for DL3. If plasma levels were lower than expected on day 4, patients were instructed to increase the rapamycin dose.

Plasma levels were then checked again on day 8 just before the start of radiotherapy. For phase II, the recommended phase II dose (RP2D) resulting from phase I was used.

\section{Patient evaluation}

During phase I acute toxicity was scored after 1 week of rapamycin single use, at the day of the last fraction of radiotherapy and weekly thereafter until 4 weeks post-operatively. Toxicity was graded according to the common toxicity criteria (NCl-CTCAE v3.0). Dose limiting toxicity (DLT) was defined as grade 4 or 5 postoperative toxicity. Secondary endpoints for phase I were incidence of other toxicity and activation status of mTOR related and dependent molecules in the tumor. During phase II, toxicity was evaluated at the same time points during treatment and every 2 weeks after treatment until resection. Secondary endpoints for phase II were tumor response, incidence of acute toxicity and changes in tumor metabolism as measured on PET scan.

\section{Treatment evaluation}

During phase II an FDG-PET-CT scan was performed before start of treatment, at day 8 after 1 week of rapamycin use only and on day 15, directly after completion of radiotherapy. A fourth scan was made around day 64 . Changes in perfusion were measured by means of a perfusion CT-scan ( $\mathrm{PCT})$, which was made with the PET-CT scanner directly after the PET-scan. The volume for perfusion measurement was located within the area of high FDG-uptake, to ensure central location within the tumor. The trans- endothelial volume transfer constant $K^{\text {trans }}$ was measured at all different time points. Technical details have been described previously [11]. PET-scans were used to analyze changes in metabolic activity. 


\section{Statistical analysis}

Differences in perfusion and changes in metabolic activity were analyzed using the Wilcoxon signed rank test. Primary endpoint for phase I was incidence of severe postoperative complications (grade 4 or grade 5 toxicity). Primary endpoint for phase II was tumor blood flow $\left(K^{\text {trans }}\right)$ assessed by perfusion CT. Secondary endpoints were incidence of acute toxicity, pathological response and metabolic response. It was hypothesized that tumor perfusion measured as $K^{\text {trans }}$ would decrease with $40 \%$ [12] with a standard deviation of 26 [13]. A sample size of 44 patients was required for a beta error of $5 \%$ and an alpha error of $10 \%$. The dropout rate was estimated to be $10 \%$, and therefore the inclusion of 47 patients was aimed for.

\section{Tissue correlative studies}

In phase I extra tumor biopsies were taken after 1 week of rapamycin monotherapy and after completion of radiotherapy. Tumor biopsies were stained immunohistochemically, for phospho-mTOR (Bioke \#2976) and phospho-4E-BP1 (Bioke \#9451), according to the manufacturer's protocol. It was hypothesized that the use of rapamycin, an mTOR inhibitor, would result in a decrease of mTOR and 4E-BP1 phosphorylation. Sections were scored by two individual pathologists.

\section{Results}

\section{Phase I - Patient characteristics}

Thirteen patients were entered in phase I of the study, between November 2006 and March 2009. Three patients were treated in DL1, 4 in DL2 and 6 in DL3. Patient characteristics are shown in table 1. In DL1 no dose adjustments were necessary at day 4 , in order to reach the planned plasma trough levels at the start of radiotherapy. In DL2 2 patients needed a dose adjustment to $6 \mathrm{mg}$ rapamycin daily. In DL3 a dose adjustment was needed in 4 patients. In 2 patients the daily dose of rapamycin was increased to $10 \mathrm{mg}$ and in 2 patients to $12 \mathrm{mg}$. 
Table 1 Patient characteristics

\begin{tabular}{|c|c|c|c|}
\hline \multicolumn{2}{|l|}{ Characteristic } & \multirow{2}{*}{$\begin{array}{l}\text { Phase I (n=13) } \\
11(85)\end{array}$} & \multirow{2}{*}{$\begin{array}{l}\text { Phase II }(n=\mathbf{3 1}) \\
21(68)\end{array}$} \\
\hline Sex & Male & & \\
\hline & Female & $2(15)$ & $10(32)$ \\
\hline Age & Median & $62[49-82]$ & 66 [44-82] \\
\hline \multirow[t]{2}{*}{ cT stage } & $\mathrm{T} 2$ & $5(38)$ & $10(32)$ \\
\hline & $\mathrm{T} 3$ & $8(62)$ & $21(68)$ \\
\hline \multirow[t]{2}{*}{ cN stage } & No & $4(31)$ & $21(68)$ \\
\hline & N1 & $9(69)$ & $10(32)$ \\
\hline \multirow[t]{3}{*}{ Type of surgery } & LAR & $10(77)$ & $24(77)$ \\
\hline & APR & $3(23)$ & $2(7)$ \\
\hline & TEM & 0 & $5(16)$ \\
\hline \multirow[t]{5}{*}{ (y)pT stage } & T0 & 0 & $1(3)$ \\
\hline & $\mathrm{T} 1$ & $1(8)$ & $3(10)$ \\
\hline & $\mathrm{T} 2$ & $6(46)$ & $17(55)$ \\
\hline & T3 & $5(38)$ & $10(32)$ \\
\hline & $\mathrm{T} 4$ & $1(8)$ & 0 \\
\hline \multirow[t]{2}{*}{ (y)pN stage } & No & $7(54)$ & $21(68)$ \\
\hline & N1 & $6(46)$ & $10(32)$ \\
\hline
\end{tabular}

Phase I - Toxicities

During the preoperative treatment with rapamycin only and radiotherapy with rapamycin no toxicities greater than grade 2 were observed. The most reported mild side effects were headache, diarrhea and stomatitis. In the postoperative phase, 2 patients suffered from a grade 3 wound infection, one patient in DL1 and one in DL3. Four patients experienced a grade 3 ileus, which persisted for $>1$ week in 1 patient (1 patient in DL1, 2 patients in DL2 and 1 in DL3). Two patients developed a grade 3 leak ( 1 in DL1 and 1 in DL2) and 1 patient was faced with a grade 4 leak (treated in DL4). The patient who had a grade 4 leak, also had a grade 4 bleeding and grade 3 colitis. In total 7 patients developed grade 3 or higher toxicities (some patients developed $>1$ toxicity). A complete overview of all toxicities $\geq$ grade 3 is given in table 2 . 
Although only one case of DLT was observed and according to the protocol DL3 could be declared the RP2D, based on the clinical judgment of the investigators the percentage of postoperative grade 3 toxicities was deemed unacceptable compared to the published toxicity profile of $5 \times 5$ Gy without rapamycin. Because wound infections and leakage may have been influenced by the immunosuppressive, protein synthesis inhibiting and anti-angiogenetic effects of rapamycin [14], it was decided in accordance with the ethical committee to postpone the time of surgery for phase II to 7-8 weeks after the completion of radiotherapy. A daily dose of $6 \mathrm{mg}$ rapamycin was declared RP2D.

Table 2 Overview of toxicities in phase I

\begin{tabular}{|c|c|c|c|c|}
\hline $\begin{array}{l}\text { Patient } \\
\text { no }\end{array}$ & $\begin{array}{l}\text { Dose level } \\
\text { rapamycin }\end{array}$ & $\begin{array}{l}\text { Dose } \\
\text { adjustment }\end{array}$ & $\begin{array}{l}\text { Adjusted } \\
\text { dose }\end{array}$ & Toxicity $\geq$ gr 3 \\
\hline 1 & $2 \mathrm{mg}$ & $\mathrm{n}$ & & gr 3 woundinfection \\
\hline 2 & $2 \mathrm{mg}$ & $\mathrm{n}$ & & gr 3 ileus \\
\hline 3 & $2 \mathrm{mg}$ & $\mathrm{n}$ & & gr 3 Gl leak \\
\hline 4 & $4 \mathrm{mg}$ & $\mathrm{y}$ & $6 \mathrm{mg}$ & \\
\hline 5 & $4 \mathrm{mg}$ & $\mathrm{n}$ & & gr 3 ileus \\
\hline \multirow[t]{3}{*}{6} & $4 \mathrm{mg}$ & $\mathrm{n}$ & & gr 3 ileus \\
\hline & & & & gr 3 GI leak \\
\hline & & & & gr 3 anorexia \\
\hline 7 & $4 \mathrm{mg}$ & $\mathrm{y}$ & $6 \mathrm{mg}$ & \\
\hline 8 & $6 \mathrm{mg}$ & $\mathrm{y}$ & $10 \mathrm{mg}$ & \\
\hline \multirow[t]{3}{*}{9} & $6 \mathrm{mg}$ & $\mathrm{y}$ & $12 \mathrm{mg}$ & gr 3 ileus \\
\hline & & & & gr 3 malabsorption \\
\hline & & & & gr 3 woundinfection \\
\hline \multirow[t]{3}{*}{10} & $6 \mathrm{mg}$ & $\mathrm{n}$ & & gr 3 colitis \\
\hline & & & & gr 4 GI leak \\
\hline & & & & gr 4 hemorrhage \\
\hline 11 & $6 \mathrm{mg}$ & $\mathrm{n}$ & & \\
\hline 12 & $6 \mathrm{mg}$ & $\mathrm{y}$ & $10 \mathrm{mg}$ & \\
\hline 13 & $6 \mathrm{mg}$ & $\mathrm{y}$ & $12 \mathrm{mg}$ & \\
\hline
\end{tabular}


Phase I - Immunohistochemistry

Tumors were stained immunohistochemically for phosphorylation of mTOR and 4E-BP1 (figure 2). For the p-mTOR staining, results were available for 7 patients. In 1 patient p-mTOR was scored as 'strong' before treatment, 'moderatestrong' in 4 patients, 'moderate' in 1 patient and 'weak-moderate' in 1 patient.
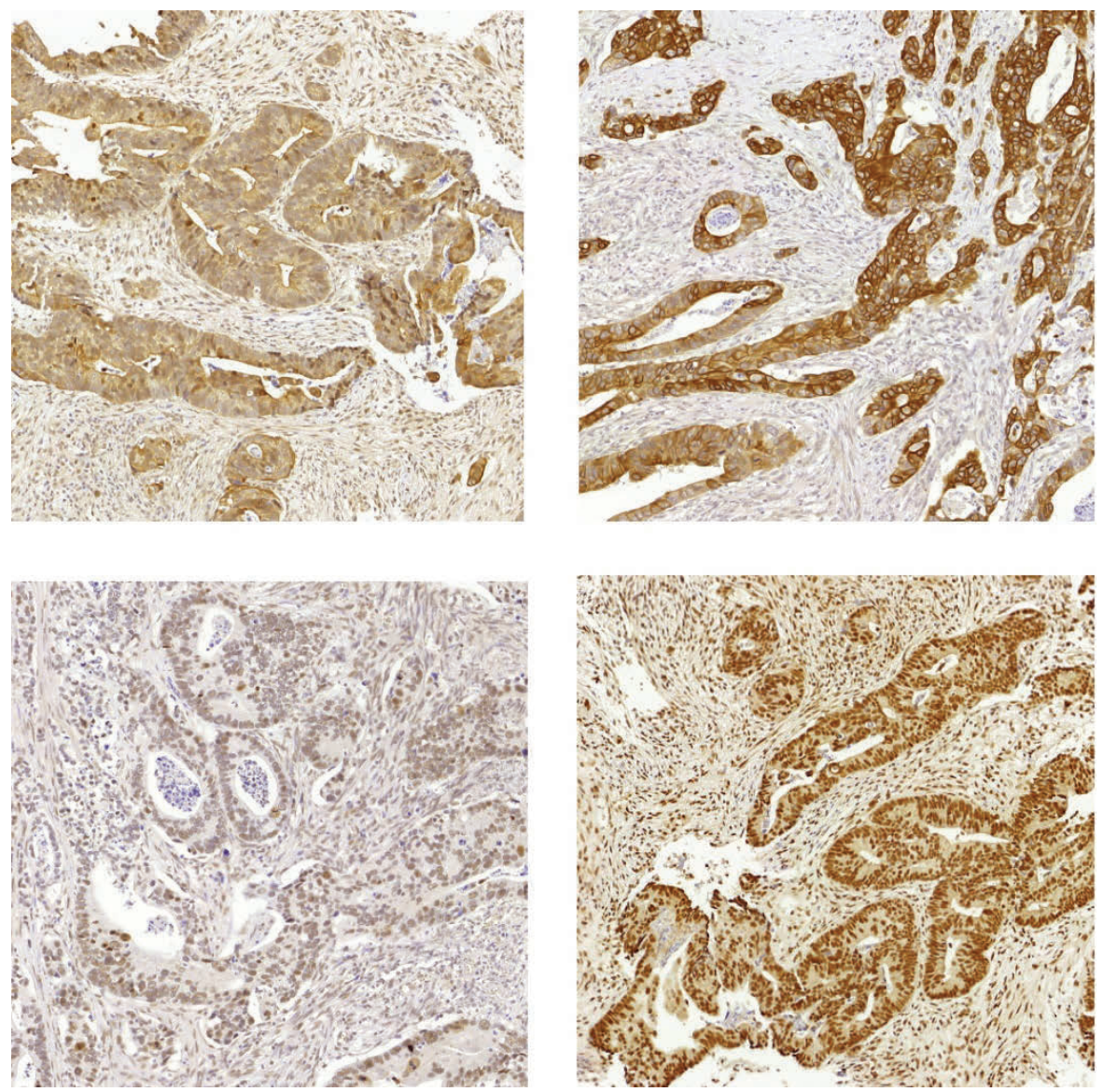

Figure 2 Immunohistochemical analysis of the phosphorylation status of mTOR (upper panels) and 4E-BP1 (lower panels) before treatment (left panels) and after treatment (right panels) on tumor biopsies of the same patient.

In 3 patients no change in phosphorylation status of mTOR was observed, in 3 patients there was a slight increase in p-mTOR and in one patient a decrease in 
p-mTOR was found. Phosphorylated 4E-BP1 data were available for 8 patients. The pre-treatment score was 'strong' in 5 patients and 'moderate-strong' in 3 patients. In 6 patients a slight decrease in p4E-BP1 status was found, while 1 patient showed a slight increase and in 1 patient a phosphorylation status remained stable. No clear correlation between changes in p-mTOR staining and p-4E-BP1 staining was observed.

\section{Phase II - Patient characteristics}

In total, thirty-one patients were entered in phase II, between April 2010 and April 2013. Inclusion was stopped because the proportion of rectal cancer patients referred for short-course radiotherapy decreased due to a change in the national guidelines and because it became clear that the primary endpoint would not be reached. Patient characteristics are shown in table 1. All patients received 6 mg rapamycin daily. In 18 patients a dose adjustment was necessary on day 4 , in order to reach the planned plasma trough levels at the start of radiotherapy. Six patients were switched to $8 \mathrm{mg}$ rapamycin daily, 8 patients to $10 \mathrm{mg}$ and 4 patients to $4 \mathrm{mg}$. The rapamycin plasma levels are depicted in figure 3 .

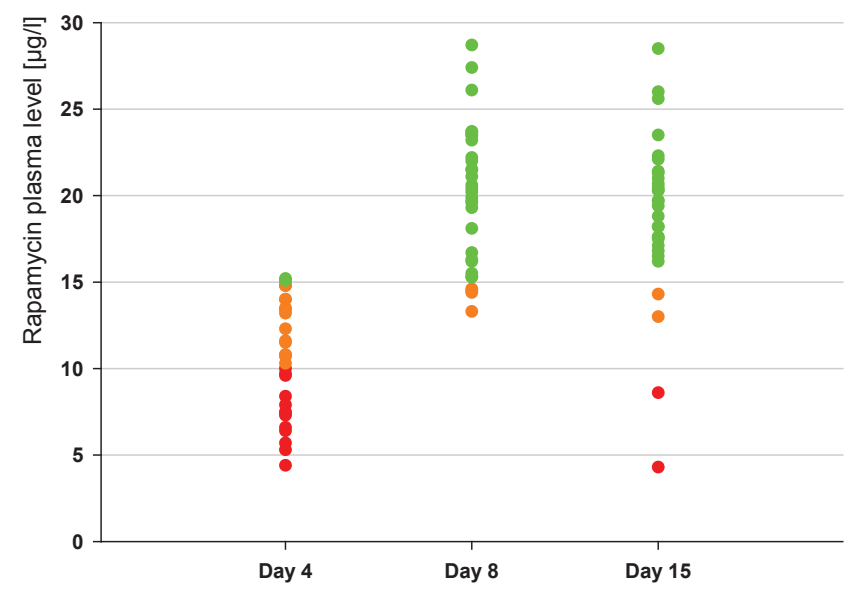

Figure 3 Rapamycin plasma trough levels measured at different time points. The level aimed at was $>15 \mu \mathrm{g} / \mathrm{l}$ (green dots). 
Phase II - Toxicities

During the first 2 weeks of treatment (rapamycin only and radiotherapy combined with rapamycin) no toxicities worse than grade 2 were reported. Two weeks after completion of radiotherapy one patient reported grade 3 diarrhea, nausea and vomiting. This was the only grade 3 or higher toxicity reported in the interval between radiotherapy and surgery and it was judged to be related to the chemotherapy that was started after completion of radiotherapy. The most frequently reported toxicities post-radiotherapy were proctitis (45\% grade 1 - 2 on day 22 and $19 \%$ on day 36 ) and diarrhea (45\% grade $1-2$ on day 22 and $35 \%$ on day 36). No severe postoperative complications have been reported in phase II.

Table 3 PET and perfusion parameters

\begin{tabular}{lllllllllll}
\hline Day & \multicolumn{1}{l}{ SUV $_{\text {max }}$} & \multicolumn{3}{c}{ PET volume } & \multicolumn{3}{c}{ Ktrans mean } \\
\hline 0 & 14.5 & $(5.2-27.9)$ & & 13.6 & $(2.10-30.2)$ & & .55 & $(.31-.82)$ & \\
8 & 12.3 & $(4.2-21.0)$ & $p=.033^{1}$ & 12.0 & $(3.1-27.9)$ & $p=.019^{1}$ & .54 & $(.30-.87)$ & $p=.92^{1}$ \\
15 & 11.8 & $(3.8-28.2)$ & $p=.113^{1}$ & 9.9 & $(1.0-25.3)$ & $p=.035^{1}$ & .51 & $(.26-.83)$ & $p=.26^{1}$ \\
64 & 6.8 & $(0-15.8)$ & $p<.001^{1}$ & 6.0 & $(0-24.8)$ & $p<.001^{1}$ & .50 & $(.40-.60)$ & $p=.72^{1}$ \\
\hline
\end{tabular}

${ }^{1}$ Wilcoxon signed rank test, SUV $\mathrm{Vax}_{\text {as }}$ compared to the scan one timepoint earlier

\section{Phase II - Perfusion and PET-response}

The results of the changes in PET-activity and tumor perfusion are summarized in table 3 and figure 4. Metabolic activity decreased significantly after 1 week of rapamycin only treatment (mean SUV max $_{14.5}$ vs. $12.3(p=.033)$ ). No further significant decrease was observed at the last day of radiotherapy (mean SUV $\max 12.3$ vs. $11.8(p=.113)$ ). On day 64 a marked further decrease in FDG-uptake was found (mean SUV max $6.8(p<.001)$ ). Tumor volume measured on PET-scan decreased gradually over time, as well after rapamycin treatment as after $5 \times 5 \mathrm{~Gy}$. Tumor perfusion, as reflected by $K^{\text {trans }}$, remained at a constant level during the entire treatment. No difference was observed in perfusion between patients who had tumor downstaging as compared to patients in whom no downstaging occurred. 


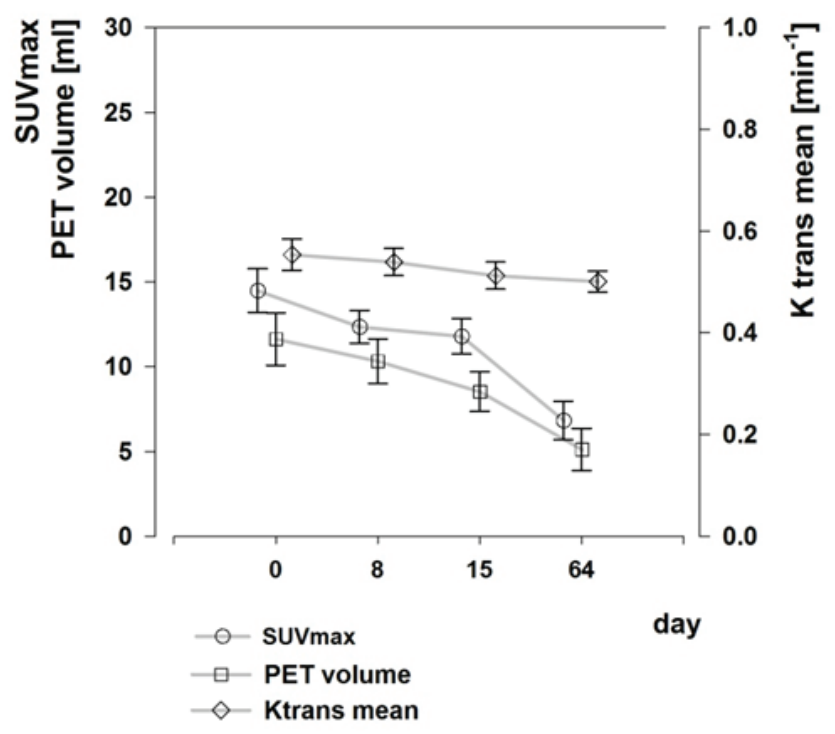

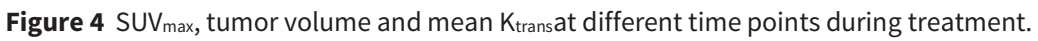

Phase II - Surgery, pathological response and local control

In 29 patients sphincter saving surgery was initially performed: a low anterior resection (LAR) in 24 patients and transanal endoscopic microsurgery (TEM) in 5 patients (table 1). In the remaining 2 patients an abdominoperineal resection (APR) was chosen. Two of the five patients who were initially treated by TEM were finally diagnosed with a ypT3 tumor, which consequently led to a LAR subsequently. One patient was diagnosed with a PCR in the resected specimen and 2 patients developed a local recurrence 5 resp. 12 months after TEM. One of these 2 patients was salvaged by chemoradiation followed by a pelvic exenteration and the second patient underwent a salvage APR. Unfortunately this last patient was recently diagnosed with a second local recurrence. Median time between end of radiotherapy and surgery was 65 days (range 45-235).

Apart from the patient with a pathological complete response in the TEMspecimen, in 3 patients the tumor was downsized to ypT1N0, resulting in a major response in $13 \%$ of patients (4 out of 31 ). Seventeen patients had a T2 tumor at the time of resection; in 4 (24\%) of them positive nodes were found in 
the resected specimen. Of the 10 patients who still had a T3 tumor after neoadjuvant treatment, 6 had positive nodes (60\%). After a median follow-up of 39 months, 3 patients (7\%) developed metastases. At the date of last follow up 2 patients had died of their metastases.

\section{Discussion}

This phase I/II trial used change in tumor perfusion as a surrogate endpoint in order to have a rapid endpoint of biological activity and to gain insight in a specific mechanism of action of rapamycin in combination with radiotherapy. This novel approach allows for a more rapid answer to specific research questions for a biological modifier, as compared to more classical endpoints such as local control and overall survival. In contrast to the hypothesis, no change in perfusion was seen, nor after rapamycin nor after combined rapamycin and radiotherapy. The combination of radiotherapy and rapamycin turned out to be safe, on the condition that surgery was delayed to 8 weeks after the end of radiotherapy.

Although the formal criteria for DLT were not met in phase I of this trial, the postoperative toxicity observed in phase I of the study was quite remarkable and deemed clinically unacceptable. Wound healing can be influenced by mTOR inhibitors by the inhibition of angiogenesis and fibroblast proliferation [14, 15]. There is some evidence from randomized trials using different immunosuppressive regimens in post-transplant patients that wound complications occur more often in patients using rapamycin as compared to patients using other types of immunosuppressants, especially in patients with high trough concentrations $(15-20 \mathrm{ng} / \mathrm{ml})$ [14, 15]. In our study, postoperative toxicities were observed at all dose levels. In phase II, after the introduction of delayed surgery, no severe postoperative toxicities were seen. Although the patient numbers do not allow firm conclusions, we think it is thus advisable to keep a gap between any neo-adjuvant treatment with mTOR inhibitors and surgery. Treatment results of short-course pre-operative radiotherapy directly followed by TME surgery for primary resectable cancer are good. The 10-year cumulative 
incidence of local recurrence for this treatment arm was 3\% in the Dutch TME trial. Overall survival at ten years was similar for the irradiated and nonirradiated group (48 vs. 49\%) [1]. Therefore it is not to be expected that addition of rapamycin leads to substantial improvement in treatment outcome when choosing this treatment approach. An alternative approach is to delay surgery to $6-8$ weeks after completion of radiotherapy $[2,3]$. This has the advantage that tumor downsizing can occur before surgery is performed and allows to choose less invasive treatment options in case of a good clinical response. Because the postoperative toxicity observed in phase I was not negligible and a relation with the study medication could not be ruled out, it was decided to treat patients in phase II with delayed surgery, in order to avoid any negative impact on wound healing. In addition, the effect of the combined treatment on tumor downsizing could be observed. Pathological complete response rates reported after 5x5 Gy and delayed surgery typically lie in the order of $8 \%[2,3,16,17]$. In this small patient group only 1 patient (3\%) developed a pCR. However, a rather strict interval between the end of radiotherapy was used in this trial, while in the majority of the published reports the exact interval was not mentioned. This may influence the $\mathrm{pCR}$ rate quite substantially. In addition, inhibition of mTOR slows down proliferation and leads to cell cycle arrest [18]. A slower proliferation may result in a slower mitotic cell death after irradiation and thus a slower tumor response. Recently, a paper reviewing the negative feedback loops that become activated as a result of mTOR inhibition, has been published. Suppression of these feedback loops leads to overactivation of upstream pathways, including PI3K, AKT and ERK. This may counteract the antiproliferative effects of mTOR inhibitors [19].

Primary endpoint of the study was a decrease in tumor perfusion as reflected by $K^{\text {trans }}$. This endpoint was not met and actually no change in $K^{\text {trans }}$ was found, neither after rapamycin run-in nor after rapamycin and radiotherapy. A possible explanation could be a mechanism that was observed after the development of this trial. $K^{\text {trans }}$ values were measured in 23 patients treated with short-course radiotherapy only (5x5 Gy) [11]. Perfusion CT imaging was performed before the start of treatment and at the day of the last fraction of radiotherapy. A 
significant increase in perfusion was observed as reflected by an increase in $K^{\text {trans }}$ from $0.36 \pm 0.11 \mathrm{~min}^{-1}$ beforetreatment to $0.44 \pm 0.13 \mathrm{~min}^{-1}$ at day $5(p<0.001)$. However, in the present study $K^{\text {trans }}$ remained unchanged during radiotherapy and the addition of rapamycin. It could therefore be hypothesized that the increase in $K^{\text {transthat }}$ was observed after 5x5 Gy without rapamycin was counterbalanced by the decrease in $K^{\text {trans }}$ expected to result from the antiangiogenic effects of rapamycin. Recently published data using everolimus, another mTOR inhibitor, also did not observe a change in tumor perfusion measured with DCE-MRI after everolimus monotherapy [20]. The influence of rapamycin on tumor vasculature was studied in a rhabdomyosarcoma mouse model [8]. In this model, a decrease in tumor microvasculature was found and an increase in oxygenation was observed already after 5 doses of rapamycin, but tumor vessel permeability only minimally changed. This raises the question whether perfusion imaging is the most suitable method to evaluate vasculature changes caused by rapamycin. However, Willett et al found the same changes in microvessel density after the administration of bevacizumab, a VEGF antibody, but they noted a clear decrease in tumor perfusion on perfusion CT [12]. $K^{\text {trans }}$ describes the transfer rate of contrast agent from the blood to the extravascular-extracellular space and is related to microvascular blood flow, vessel wall permeability and vessel density. This means that this constant is influenced by different aspects of tumor vasculature. A pre-clinical study looked at the specific anti-angiogenic mechanisms of an mTOR inhibitor (everolimus) as compared to a VEGFR tyrosine kinase inhibitor [21]. They described that alterations in tumor vascular biology were partly caused by comparable mechanisms but differences in the vascular response were also observed. In the mouse model of this study, $K^{\text {trans }}$ as measured with DCE MRI remained constant during mTOR treatment, despite a clear tumor response, but a clear decrease in $K^{\text {trans }}$ was seen after treatment with a VEGFR inhibitor. Apparently, different ways of anti-angiogenic treatment result in different types of vasculature changes and this translates into different changes on imaging. Furthermore, responses of tumor vasculature to mTOR inhibitors can be quite heterogeneous possibly depending on differences in the tumor microenvironment [22]. 
In contrast to the perfusion scans, a significant decrease in metabolic activity was seen on PET-CT. This observation is in line with the findings of Ciunci et al in their phase I trial with everolimus and cetuximab [20]. In the run-in phase of everolimus a mean decrease in SUV $_{\max }$ of $24 \%$ was seen. Other clinical and preclinical studies showed the same results. Recently a quick and clear decrease in FDG uptake was demonstrated under mTOR1/2 inhibition in a mouse glioma model [23]. In a preclinical model of cisplatin-resistant ovarian cancer, treatment with a dual PI3K/mTOR inhibitor resulted in a significant decrease of FDG-uptake, which correlated with a decrease in proliferation and inhibition of the PI3K/mTOR pathway [24]. In a mouse model in which colon tumors are initiated by a dominant active PI3K, a clear response was seen on PET-scan after treatment with rapamycin, but not after placebo treatment [25]. Honer et al used different cell lines in a mouse model: cell lines that were in vitro characterized as sensitive to everolimus and cell lines that were insensitive. The cell lines that were insensitive in vitro did show growth inhibition but no changes in FDG metabolism were observed, while in the sensitive tumor model a clear decrease of FDG-uptake was observed [26]. The authors hypothesize that this may be explained by a different mechanism of action, namely the anti-angiogenic action of everolimus, and that the change in FDG-metabolism is caused by other mechanisms. In a study with patients with metastatic renal cell cancer, a decrease in FDG-uptake was found after treatment with everolimus [27]. This early metabolic change was correlated with change in tumor burden.

In conclusion, rapamycin turned out to be safe in combination with shortcourse hypofractionated radiotherapy in rectal cancer treatment, but it is advisable to postpone surgical treatment until 8 weeks after combined treatment to avoid a possible increase in postoperative complications. Although rapamycin is thought to cause changes in tumor vasculature, this does not translate in changes in Ktrans in vivo. Rapamycin has a biological influence on rectal cancer as reflected by the changes in FDG-uptake. In this patient cohort no clear increase in tumor response was observed after combined radiotherapy and rapamycin. 


\section{References}

[1] van Gijn W, Marijnen CA, Nagtegaal ID, Kranenbarg EM, Putter H, Wiggers T, et al. Preoperative radiotherapy combined with total mesorectal excision for resectable rectal cancer: 12-year follow-up of the multicentre, randomised controlled TME trial. Lancet Oncol. 2011;12:575-82.

[2] Hatfield P, Hingorani M, Radhakrishna G, Cooper R, Melcher A, Crellin A, et al. Short-course radiotherapy, with elective delay prior to surgery, in patients with unresectable rectal cancer who have poor performance status or significant co-morbidity. Radiother Oncol. 2009;92:210-4.

[3] Radu C, Berglund A, Pahlman L, Glimelius B. Short-course preoperative radiotherapy with delayed surgery in rectal cancer - a retrospective study. Radiotherapy and oncology : journal of the European Society for Therapeutic Radiology and Oncology. 2008;87:343-9.

[4] Maas M, Beets-Tan RG, Lambregts DM, Lammering G, Nelemans PJ, Engelen SM, et al. Waitand-see policy for clinical complete responders after chemoradiation for rectal cancer. Journal of clinical oncology : official journal of the American Society of Clinical Oncology. 2011;29:4633-40.

[5] Shinohara ET, Cao C, Niermann K, Mu Y, Zeng F, Hallahan DE, et al. Enhanced radiation damage of tumor vasculature by mTOR inhibitors. Oncogene. 2005;24:5414-22.

[6] LoPiccolo J, Blumenthal GM, Bernstein WB, Dennis PA. Targeting the PI3K/Akt/mTOR pathway: effective combinations and clinical considerations. Drug Resist Updat. 2008;11:32-50.

[7] Manegold PC, Paringer C, Kulka U, Krimmel K, Eichhorn ME, Wilkowski R, et al. Antiangiogenic therapy with mammalian target of rapamycin inhibitor RAD001 (Everolimus) increases radiosensitivity in solid cancer. Clin Cancer Res. 2008;14:892-900.

[8] Myers AL, Orr WS, Denbo JW, Ng CY, Zhou J, Spence Y, et al. Rapamycin-induced tumor vasculature remodeling in rhabdomyosarcoma xenografts increases the effectiveness of adjuvant ionizing radiation. J Pediatr Surg. 2012;47:183-9.

[9] Fokas E, McKenna WG, Muschel RJ. The impact of tumor microenvironment on cancer treatment and its modulation by direct and indirect antivascular strategies. Cancer Metastasis Rev. 2012;31:823-42.

[10] Jain RK. Normalizing tumor vasculature with anti-angiogenic therapy: a new paradigm for combination therapy. Nat Med. 2001;7:987-9.

[11] Janssen MH, Aerts HJ, Kierkels RG, Backes WH, Ollers MC, Buijsen J, et al. Tumor perfusion increases during hypofractionated short-course radiotherapy in rectal cancer: sequential perfusion-CT findings. Radiother Oncol. 2010;94:156-60.

[12] Willett CG, Boucher Y, di Tomaso E, Duda DG, Munn LL, Tong RT, et al. Direct evidence that the VEGF-specific antibody bevacizumab has antivascular effects in human rectal cancer. Nat Med. 2004;10:145-7.

[13] de Lussanet QG, Backes WH, Griffioen AW, Padhani AR, Baeten Cl, van Baardwijk A, et al. 
Dynamic contrast-enhanced magnetic resonance imaging of radiation therapy-induced microcirculation changes in rectal cancer. Int J Radiat Oncol Biol Phys. 2005;63:1309-15.

[14] Nashan B, Citterio F. Wound healing complications and the use of mammalian target of rapamycin inhibitors in kidney transplantation: a critical review of the literature. Transplantation. 2012;94:547-61.

[15] Pengel LH, Liu LQ, Morris PJ. Do wound complications or lymphoceles occur more often in solid organ transplant recipients on mTOR inhibitors? A systematic review of randomized controlled trials. Transpl Int. 2011;24:1216-30.

[16] Latkauskas T, Pauzas H, Gineikiene I, Janciauskiene R, Juozaityte E, Saladzinskas Z, et al. Initial results of a randomized controlled trial comparing clinical and pathological downstaging of rectal cancer after preoperative short-course radiotherapy or long-term chemoradiotherapy, both with delayed surgery. Colorectal Dis. 2012;14:294-8.

[17] Pettersson D, Holm T, Iversen H, Blomqvist L, Glimelius B, Martling A. Preoperative shortcourse radiotherapy with delayed surgery in primary rectal cancer. Br J Surg. 2012;99:577-83.

[18] Strimpakos AS, Karapanagiotou EM, Saif MW, Syrigos KN. The role of mTOR in the management of solid tumors: an overview. Cancer Treat Rev. 2009;35:148-59.

[19] Rozengurt E, Soares HP, Sinnet-Smith J. Suppression of Feedback Loops Mediated by PI3K/mTOR Induces Multiple Overactivation of Compensatory Pathways: An Unintended Consequence Leading to Drug Resistance. Molecular cancer therapeutics. 2014;13:2477-88.

[20] Ciunci CA, Perini RF, Avadhani AN, Kang HC, Sun W, Redlinger M, et al. Phase 1 and pharmacodynamic trial of everolimus in combination with cetuximab in patients with advanced cancer. Cancer. 2014;120:77-85.

[21] Lane HA, Wood JM, McSheehy PM, Allegrini PR, Boulay A, Brueggen J, et al. mTOR inhibitor RAD001 (everolimus) has antiangiogenic/vascular properties distinct from a VEGFR tyrosine kinase inhibitor. Clin Cancer Res. 2009;15:1612-22.

[22] Weppler SA, Krause M, Zyromska A, Lambin P, Baumann M, Wouters BG. Response of U87 glioma xenografts treated with concurrent rapamycin and fractionated radiotherapy: possible role for thrombosis. Radiother Oncol. 2007;82:96-104.

[23] Keen HG, Ricketts SA, Maynard J, Logie A, Odedra R, Shannon AM, et al. Examining Changes in [(18) F]FDG and [ (18) F]FLT Uptake in U87-MG Glioma Xenografts as Early Response Biomarkers to Treatment with the Dual mTOR1/2 Inhibitor AZD8055. Mol Imaging Biol. 2014;16:421-30.

[24] Lheureux S, Lecerf C, Briand M, Louis MH, Dutoit S, Jebahi A, et al. (18)F-FDG Is a Surrogate Marker of Therapy Response and Tumor Recovery after Drug Withdrawal during Treatment with a Dual PI3K/mTOR Inhibitor in a Preclinical Model of Cisplatin-Resistant Ovarian Cancer. Transl Oncol. 2013;6:586-95.

[25] Deming DA, Leystra AA, Farhoud M, Nettekoven L, Clipson L, Albrecht D, et al. mTOR inhibition elicits a dramatic response in PI3K-dependent colon cancers. PLoS One. 2013;8:e60709. 
[26] Honer M, Ebenhan T, Allegrini PR, Ametamey SM, Becquet M, Cannet C, et al. AntiAngiogenic/Vascular Effects of the mTOR Inhibitor Everolimus Are Not Detectable by FDG/FLT-PET. Transl Oncol. 2010;3:264-75.

[27] Chen JL, Appelbaum DE, Kocherginsky M, Cowey CL, Rathmell WK, McDermott DF, et al. FDGPET as a predictive biomarker for therapy with everolimus in metastatic renal cell cancer. Cancer Med. 2013;2:545-52. 
Chapter 9

General discussion and future perspectives 
Results of rectal cancer treatment have improved substantially during the last 25 years. Patients diagnosed with rectal cancer in 1990 had a 5-year overall survival of approximately 50\%, while patients who were treated in 2010 had a $65 \%$ chance of being alive 5 years later [1]. This improvement in treatment outcome can be ascribed mainly to improvements in surgical techniques. The most important contribution of preoperative radiotherapy is an improvement in local control. Although one would expect that better local control ultimately translates in better overall survival, randomized trials do not support this [2-5]. Considering the local recurrence rates of around 5\%, it is not to be expected that these results can be improved. Although the relative risk reduction of preoperative treatment is $50 \%$ in all subgroups, the estimated absolute reduction of local recurrence varies from 1 to $10 \%$ [5]. This suggests that the optimum in local treatment has been reached and that a risk of overtreatment is present.

This observation makes it more essential to take into account the toxicity caused by the different treatment components. Although short course preoperative radiotherapy followed by an immediate resection usually does not result in any acute toxicity, it has been shown to add toxicity compared to surgery only. In the Dutch TME trial, toxicity and quality of life have been evaluated carefully. Patients who were treated with radiotherapy and surgery had a higher defecation frequency, fecal soiling and more sexual problems, although this did not translate in a decrease in overall functioning [6, 7]. It is important to keep in mind that long term toxicities of surgery and radiotherapy are overlapping and that it is difficult to separate them. Hence, there are two strategies to minimize treatment related toxicity: 1 . a stricter selection of patients who will benefit from pre-operative treatment and 2. the omission of surgery or the use of less invasive surgical techniques in selected patients. The work presented in this thesis can be seen as steps towards a better informed choice between those two strategies (part II of the thesis) and a higher chance to fit in the second strategy (part I and III).

Part 1, devoted to the role of PET-CT in the delineation of rectal tumors, describes methods that can be used to adequately identify the volume of the primary tumor, which makes it possible to give an integrated boost in order to 
increase the chance of developing a complete response. The prediction models described in part 2 are a first step towards early selection of patients more likely to respond to neoadjuvant treatment. The clinical trials presented in part 3 were aimed at treatment intensification in order to increase the proportion of patients developing a complete response, making it possible to select them for a wait and see protocol without a surgical resection.

\section{Organ preservation in rectal cancer}

Before discussing the results presented in this thesis, it is necessary to discuss briefly the current status of organ preserving strategies in rectal cancer. Although organ preservation sounds as an attractive alternative to major surgery, many questions about oncological safety and functional outcome remain to be answered.

The only patient group that is suitable for organ preserving surgery according to the Dutch guidelines consists of patients with a good risk T1 tumor ( $T 1$, maximum diameter $4 \mathrm{~cm}$, grade 1-2, no lymfangio-invasion). In all other patients, organ sparing approaches must be seen as experimental.

For patients who develop a clinical complete response (CCR) after neoadjuvant treatment, a wait and see approach could be an option. However, the definition of $\mathrm{CCR}$ is difficult and differs between the groups who reported on this strategy $[8,9]$. The concordance rates between $C C R$ as scored pre-operatively and pCR as described by the pathologist after resection are variable and disappointing in several studies [10]. Combination of different modalities (digital rectal examination, endoscopy and MR with diffusion weighted imaging) seems to be the best way to evaluate response to neoadjuvant treatment, but an experienced team is needed to interpret the findings of these examinations. The addition of imaging is very important, not only for the evaluation of the response of the primary tumor, but also for the evaluation of nodal status. An alternative for a watch and wait approach after preoperative treatment is to perform a TEM procedure, which can be seen as a diagnostic and therapeutic procedure. It allows adequate T-staging and removes residual tumor. Although 
$\mathrm{N}$-staging is not possible, the ypT-stage can be used to estimate the risk of remaining positive nodes, since there is a clear relationship between T-stage after neoadjuvant treatment and the risk of positive lymph nodes (ranging from $<5 \%$ for ypT0 tumors to $>20 \%$ in ypT3-4 tumors) [11-13]. A possible disadvantage of this approach is the concern that the results of completion surgery after TEM may be worse than the results of primary TME surgery [14] although other studies do not report a worse outcome [15-18]. An Italian trial randomized between TEM and TME after chemoradiation in patients with lowlying CT2NO rectal tumors [19]. Local recurrence rates were not statistically significant between both arms ( $8 \%$ after TEM and 6\% after TME). However, the recurrence rate in the TME arm was rather high, taking into account that only small tumors were included and treated pre-operatively with chemoradiation. In two other phase II trials [17, 20], the decision to perform a completion resection was based on predefined criteria. In general, patients who were treated according to the protocol (i.e. completion TME in case of adverse histopathological findings) had a good outcome, but the local recurrence rates were rather high in patients who did not undergo completion surgery. These findings confirm that local surgery after chemoradiation in high-risk patients is an inadequate treatment.

In case of a "watch and wait" approach, patients are followed with frequent imaging and endosocopy. The tumor is expected to recur in a proportion of patients, since it will be very difficult to develop a decision algorithm with $100 \%$ specificity for CCR. Therefore, local recurrence rate is not the best endpoint to evaluate organ sparing treatments. In the series of Habr Gama et al a 68\% 5year disease free survival was reported in a watch and wait protocol, but this number improved to $94 \%$ if the first recurrence was not take into account [21]. As long as salvage surgery is possible, a local recurrence can be seen as a calculated risk. However, it has to be proven that this approach, leaving residual tumor in place for a longer time after chemoradiation than in the case of immediate surgery, does not result in a higher chance of distant metastases. In the Habr-Gama series the occurrence of distant metastases did not differ significantly between patients who developed a local recurrence and who did 
not [21]. This question will be very difficult to answer and ideally a randomized trial including radical surgery as a treatment arm should be performed.

\section{PET-CT for tumor delineation in rectal cancer}

PET-CT was hypothesized to be a reliable tool for tumor delineation in rectal cancer treatment, leading to a decrease in interobserver variation.

PET-CT scan has gained a lot of attention in the past decade among radiationoncologists. Particularly ${ }^{18}$ FDG-PET scans have been proven to be very useful not only for staging, but also for response prediction, treatment evaluation and tumor delineation $[22,23]$. The use of PET-CT as a tool for tumor delineation has been studied principally in head and neck cancer [24] and lung cancer [25]. In these two sites, the benefit of PET-CT is more obvious than in rectal cancer, because radiotherapy is often used as a curative treatment in lung and head and neck cancer, whereas in rectal cancer, radiotherapy is considered an adjuvant treatment to surgery. Consequently, lower radiation doses are used for rectal cancer treatment and a large elective volume is treated, which makes it less critical to exactly define the GTV. However, for reasons stated above (limitation of toxicity, dose escalation), PET-CT could be of benefit for treatment planning in rectal cancer as well.

The study described in chapter 2 was essential to know whether PET-CT could be used as a reliable tool for delineation purposes in rectal cancer. The use of a source-to-background ratio-based (SBR) auto-delineation algorithm resulted in a very good correlation between tumor dimensions measured by PET-CT and measured by the pathologist in lung and head and neck cancer [26, 27]. A big advantage of automatic delineation algorithms is the absence of human influence, which introduces inter- and intraobserver variation. This has been confirmed in the work presented in chapter 2: the strongest correlation with pathology results was found for PET-based auto-contouring and the correlation was less strong when observers were involved. The use of PET-CT, nevertheless, has some potential pitfalls. The SBR method as used in chapter 2 depends on a correct calibration of the PET-scanner and this calibration has to be done 
separately for each scanner. A second point of attention is the occurrence of peri-tumoral inflammation, which can influence the dimensions measured on PET-CT. Furthermore one has to realize that PET-CT, like any other imaging modality, is not able to detect any microscopic extension of the tumor. A specific caveat for rectal tumors when using PET-based automatic delineation has to do with the anatomy of the rectum, which lies in close proximity to the bladder. Due to the high concentration of FDG in urine it may be difficult to separate the tumor contour from the bladder and a manual check or predefinition of a region of interest may be necessary.

Apart from the SBR method, other automatic delineation algorithms exist [28, 29]. Recently Withofs et al published the results of a comparison of 6 different PET-based GTVs [30]. Four GTVs were created with 2 different commercially available software systems and based on fixed thresholds (SUV 2.5 and 45\% of SUVmax), one contour was based on the fuzzy locally adaptive Bayesian algorithm (FLAB) and for the sixth contour a watershed transform and cluster analysis was used. They concluded that contours produced by the different algorithms differed significantly from each other. Even contours created with the same method in another software package were not exactly equal. These findings illustrate that the results that are obtained from PET-based algorithms are helpful but should be interpreted with caution. Nevertheless, the reproducibility of an automated delineation algorithm appears to be much better than for manual PET-based delineation [31]. One should realize that the complete chain influences the outcome of the automatic contour: not only the scanner hardware, but also the reconstruction algorithms used for CT and PET, the delineation software and the transfer to the planning software. It is therefore very important to have a good quality assurance system before automatic delineation can be introduced clinically in a department.

The question is whether all these considerations and variations in PETtechnique and delineation algorithms are of great importance in clinical practice for the treatment of rectal cancer. We have shown that GTVs and the intra-observer variation were significantly smaller with the use of PET (chapter 3). This gain in accuracy is partly lost by the wide margins that are needed to 
compensate for the uncertainties when treating rectal tumors. As the rectum is a hollow organ and it lies in close proximity to the bladder, there is a large day to day variation in organ filling and organ position [32]. This makes the use of rather big PTV margins to account for this organ motion imperative and makes the potential role of PET different from its role in other tumor sites. However, what remains critical in tumor delineation is a reproducible and reliable identification of the primary tumor and this may even become more important in the context of dose escalation in selected patients.

The hypothesis that PET-CT is an adequate and useful method for tumor delineation in rectal cancer has been confirmed in these 2 studies.

\section{Response prediction in rectal cancer}

It was hypothesized that response prediction in rectal cancer treatment was possible by means of multimodality predictive modeling.

In current clinical practice most oncological treatments are based on national guidelines. This has the advantage that treatments are more or less comparable between different hospitals and that treatment choices are evidence based. A disadvantage is that treatment guidelines generally are not detailed enough to help with treatment individualization and that the process of writing and implementation is rather slow, so that it is difficult to introduce the most recent developments. Although in the most recent update of the Dutch guideline for rectal cancer treatment, a first step towards tailoring of treatment was attempted through the identification of three subgroups of rectal cancer patients who need different treatments, this is still a very rough division (figure 1). Moreover, this division is based on tumor extension in the surrounding tissues and nodal status. Especially the prediction of nodal status is still cumbersome.

The development of prediction models can help the clinician make better supported and individualized treatment choices. The first question is what the goal of the treatment should be. If radiotherapy is seen only as an adjunct to surgery in order to ameliorate local control (which is the more "classical" 
approach), the prediction model should be aimed at the identification of patients who will benefit from pre-operative treatment versus patients who may go for immediate resection and the differentiation between patients who need neoadjuvant chemoradiation versus patients in whom short-course radiotherapy suffices. Another possible goal of response prediction is to enhance the chance of developing a pathological complete response, in order to maximize the chance of organ saving treatment. On the one hand this may help to identify patients who will benefit from treatment intensification, and on the other hand save patients who have a very low chance of developing a complete response from a potential toxic treatment. In order to be able to influence treatment decisions, these models need to be based on variables that are available at the time of diagnosis or early during treatment.

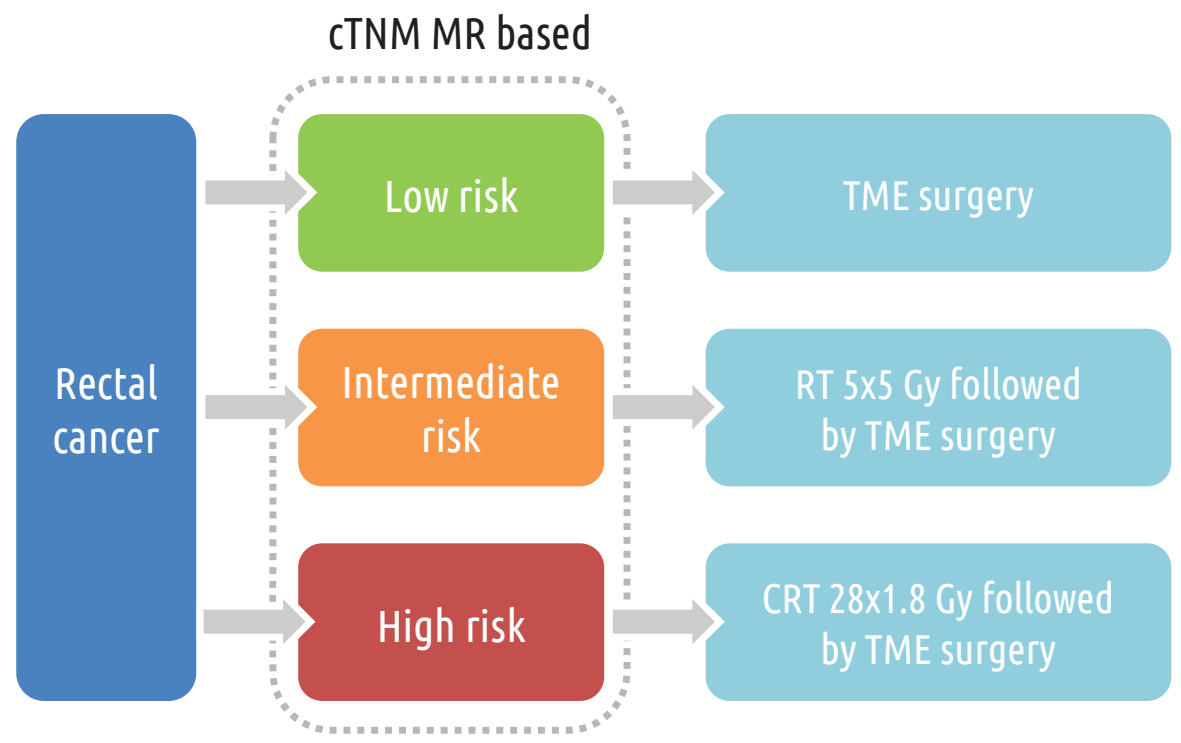

Figure 1 Treatment of rectal cancer according to the Dutch national guidelines.

Until now, most research has focused on PET-scan as a possible predictor. A recent meta-analysis identified 31 studies using PET-scan for response prediction in rectal cancer [33]. Early changes on scans made during treatment 
as well as changes on scans made after treatment can both be useful in clinical practice. Early scans make it possible to adapt treatment (e.g. add a boost for patients in an intermediate risk group) and scans made after the completion of chemoradiation can help in the decision making for surgical treatment (e.g. proceed to TME surgery in case of a partial response, consider local excision for a near complete response and discuss a wait and see approach in clinical complete responders).

Chapter 4 describes the development of a prediction model incorporating PET information before and after treatment. What makes this model different from most other papers on PET and response prediction is the relatively large sample size, the multivariate methods used and the validation of the models. The model based on the maximal SUV before treatment, the relative change in $S_{S U} V_{\max }$ and tumor length proved to be the most predictive. Relative changes in SUV $_{\max }$ have been reported by other authors to be predictive for response. $\mathrm{SUV}_{\max }$ as a single parameter is not strong enough to predict outcome [34-36]. Other imaging techniques are emerging rapidly, of which magnetic resonance imaging (MRI) is the most promising technique [37]. An advantage of MRI over PET is the better spatial resolution. Modern MRI techniques, like dynamic contrast enhanced MR (DCE-MR) and diffusion-weighted imaging (DWI), may significantly increase the predictive value of this imaging modality. While rather strong response predictions as well early during treatment as after treatment completion have been reported for DWI imaging [38-41], the results and added value of DCE-MR are more variable $[42,43]$. One study reported on the combination of PET and MR data, showing that combining both modalities resulted in a stronger predictive value of response [44].

The use of other biological markers may have added value compared to prediction models based on imaging data. In chapter 5 a model that uses blood biomarkers is introduced. Blood biomarkers are potentially attractive because they can be collected easily, measurement is relatively cheap and easy and, in contrast to for example genetic biomarkers in tumor cells, they are less influenced by tumor heterogeneity. The study presented in this thesis is the first one that tested a panel of 9 blood biomarkers with potential predictive value. 
CEA turned out to be a predictor for complete response and good response in this multivariate model. CEA has been shown in several other studies to be of predictive value for response to chemoradiation in rectal cancer [45-47]. Interleukin-8 was also predictive for response to chemoradiation. Although previous reports describe an association between IL-8 polymorphisms and nodal involvement and recurrence in rectal cancer, our work was the first to demonstrate an association with response to chemoradiation. Although this study is a proof of principle that blood biomarkers have additional value to clinical and imaging derived parameters, external validation is necessary before this model can be implemented in clinical practice. In this validation process, monitoring the effect of using blood sample analyses from different laboratories will be important, since the measurements for this study were all done in one laboratory, using the same technique for each biomarker.

While blood biomarkers give more global information about tumor biology, molecular markers can provide more detailed information. The review presented in chapter 6 summarizes the available evidence regarding biochemical and molecular biological factors that could help with treatment decision making in rectal cancer. Predictive as well as prognostic factors are described. A prognostic factor gives information about the risk of recurrence of disease irrespective of a certain treatment, while a predictive factor predicts the chance that a patient will benefit from a treatment. The general conclusion is that the value of this type of markers is currently limited. More recently several specific genetic alterations and microRNA expression patterns that have a predictive value for response in rectal cancer have been published [48-51]. Although this shows that very specific changes in rectal tumors may play a role in response prediction, the field of genomics in rectal cancer is still in its infancy. A difficulty of the genomic approach is the influence of tumor heterogeneity. Especially in advanced rectal tumors, a small biopsy, used for diagnosis, may not be representative for tumor biology. The biochemical and genetic factors that have been tested until now were mainly isolated features, not tested multivariately.

The hypothesis that multimodality models can predict response to neoadjuvant treatment holds true for PET-based information and blood biomarkers. Until 
now, biochemical and genetic factors have limited value in response prediction, but there is certainly a need for further testing of this category of markers in a multivariate and multimodality setting.

\section{Combined treatment in rectal cancer}

For the clinical trials presented in this thesis we hypothesized that the combination of AKT inhibition and radiotherapy and mTOR inhibition and radiotherapy would lead to an increased tumor response in rectal cancer.

Combined modality treatment in rectal cancer has a long history. The first trials combining radiotherapy and chemotherapy date back to the 80's [52]. This combination has been proven to be more active than radiotherapy alone in advanced rectal cancer in terms of local control [53]. However, no significant difference in disease free and overall survival has been shown. The cornerstone of chemoradiation in the treatment of rectal cancer is fluorouracil. In the past years the addition of oxaliplatin to fluorouracil and radiotherapy has been tested. Although in one study an increase in response was found [54], two other studies reported comparable responses and an increase in toxicity in the oxaliplatin arm $[55,56]$. The combination of fluoropyrimidine-based chemotherapy with irinotecan has been studied in several phase II studies [5762]. Except for one study, PCR rates were not significantly higher than the rates reported after chemoradiation with 5-FU or capecitabine alone and acute toxicity rates, especially diarrhea, were generally higher.

More recently, targeted biological strategies have been tested in phase I and II trials. A potential advantage of adding targeted agents over adding another cytostatic drug, is the avoidance of overlapping toxicities. On the other hand, it may be a challenge to identify patients who will benefit from the addition of a targeted agent, because these drugs often only have effect when certain mutations are present or absent in a tumor. We know from treatment of colorectal cancer in the palliative setting for example, that patients who have a KRAS mutated tumor do not benefit from anti-EGFR treatment [63]. After the landmark phase III study showing an improved overall survival in head and neck 
cancer patients for the combination of radiotherapy and the EGFR-inhibitor cetuximab as compared to radiotherapy alone [64], EGFR-inhibition was tested also in rectal cancer treatment [65-71]. The results of these trials are quite variable, with the majority of them showing a disappointing percentage of $\mathrm{PCR}$. The predictive value of KRAS status was less unequivocal than in the studies combining chemotherapy and cetuximab in colon cancer. However, a pooled analysis of 4 phase II trials identified EGFR and VEGF mRNA expression levels and KRAS mutation status as predictive markers for response to cetuximabbased chemoradiation [72]. Thus, although the results of the addition of cetuximab are disappointing, it is possible that a subgroup of patients will benefit from it. It has also been suggested that timing of chemotherapy, radiotherapy and EGFR-inhibition may be critical [73]. A third possible explanation of a negative interaction between EGFR-inhibitors and chemoradiation is the slowing down of the cell cycle that is caused by EGFR inhibition, as the additive effect of 5-FU is cell cycle dependent [74].

Another interesting target in combined therapy for rectal cancer is vascular endothelial growth factor (VEGF). The most used agent is bevacizumab, a monoclonal antibody against VEGF. No phase III trials have been published, but multiple phase II trials have reported encouraging results with respect to tumor response [75-88]. Complete response rates vary from 13 to $37 \%$, but the pooled pCR rate is $21 \%$, which is comparable to conventional chemoradiation. Moreover, the reported postoperative toxicity rates are worrisome.

These two examples of targeted agents that are added to existing chemoradiation schedules, are an illustration of unexpected responses one may observe in the translation of new drugs from bench to bedside [89]. In this thesis, 2 early translational trials in rectal cancer are presented. A special characteristic of these phase I/II trials is the use of existing drugs that have been used clinically for other indications than cancer. Possible advantages of this 'drug repurposing' are the well-known toxicity profile and the relatively low costs as compared to newly developed targeted drugs. Nelfinavir was developed as a protease inhibitor for the treatment of the human immunodeficiency virus (HIV). Its potential role in the treatment of cancer was 
discovered at the beginning of this century, when it was found that protease inhibitors could inhibit the PI3K/Akt pathway, which plays a central role in cell cycle survival [90]. PI3K is a downstream messenger of EGFR amongst others. Therefore, in theory nelfinavir could also work in EGFR-mutated tumors. The phase I trial testing the addition of nelfinavir to capecitabine-based chemoradiation (chapter 7 ) proved the feasibility of this combination. Yet, the toxicity profile of nelfinavir, with diarrhea as a frequent side effect, seemed to be less favorable for this specific combination. Furthermore, plasma levels of nelfinavir turned out to be quite variable in clinical practice, which makes it less attractive to use in daily clinical practice. The use of nelfinavir for HIV-treatment was discontinued after the completion of this phase I trial and the drug no longer available in the Netherlands. This made us decide to abolish phase II of this trial.

The second clinical trial described in this thesis, investigated the safety and efficacy of the addition of rapamycin, an inhibitor of mTOR, to short course radiotherapy (chapter 8). Rapamycin has been used for over 2 decades as an immunosuppressant in patients who underwent renal transplantation. The mTOR pathway plays a central role in cell survival and activation leads to prosurvival signals. It influences protein synthesis, lipid synthesis, autophagy and energy metabolism. It has been known for a long time that inhibition of mTOR inhibits tumor growth [91]. Inhibition of mTOR can overcome radioresistance $[92,93]$. In phase I of the trial, surgery was planned immediately after the end of radiotherapy with rapamycin. Change in tumor perfusion was chosen as a "modern" alternative primary endpoint. The combination treatment was well tolerated and only one patient experienced a dose limiting toxicity in the postoperative period. However, a remarkable number of patients experienced grade 3 complications after surgery. The immunosuppressive action of rapamycin, which can lead to impaired wound healing, was suspected to be causing these complications. Therefore, surgery was postponed to 8 weeks after $\mathrm{RT}+$ rapamycin in phase II. The hypothesized decrease in tumor perfusion as a result of rapamycin was not confirmed: perfusion remained constant during the entire treatment period. However, on PET-scan a decrease in metabolic volume 
as well as SUV $V_{\max }$ was found after rapamycin treatment, indicating a biological effect of the drug in rectal cancer. Based on these data it cannot be ruled out that rapamycin is effective, but further studies are needed to investigate the most optimal timing and combination of mTOR inhibition, possible other drugs and radiation schemes.

The hypothesis as formulated prior to the start of these two clinical trials cannot be proven nor rejected on the basis of these results. The combination of radiotherapy, capecitabine and nelfinavir showed an unfavorable toxicity profile, therefore this trial was discontinued after phase I. Despite promising response rates, the patient group was too small to draw conclusions regarding efficacy. In the rapamycin trial, the primary endpoint was not reached and the response rate was not higher than in series using the same regimen without rapamycin. Imaging, however, demonstrated that rapamycin has biological activity in rectal cancer.

\section{Future perspectives}

Until recently, rectal cancer therapy was characterized by a tendency to intensify treatment. However, a shift towards individualization of treatment and less aggressive approaches can be observed during the last years. It has become clear that rectal cancer, as almost all other cancer types, is not "one disease" but a spectrum of diseases with large differences in biological behavior. In the evolution of the treatment of rectal cancer, one can draw a parallel with breast cancer treatment. William Halsted (1852-1922) introduced the principle of radical mastectomy, which led to a dramatic improvement in treatment results. This opened the way to more and more radical surgery, with the hypothesis that an aggressive surgical locoregional treatment would be able to remove all sources of metastases. In the second half of the $20^{\text {th }}$ century there was a reversal, towards less radical and less mutilating treatment and it was shown that breast conserving treatment is a safe option for a large group of patients with equivalent outcome. Finally, in recent years it has become clear that an axillary lymph node dissection, which was standard for all patients with breast 
cancer, could be safely omitted in patients with a negative sentinel node. The most recent insights show that it is even safe to leave the axilla untreated in some patients, even in case of a positive sentinel node.

In rectal cancer, as described in the introduction of this thesis, Bill Heald recognized the importance of good radical surgery and its impact on local control and survival. Several large trials then showed the added value of radiotherapy or chemoradiation to TME-surgery. Trials followed which investigated intensification of local treatment in order to maximize response. And since the beginning of this century it was noticed that in some cases that respond well to pre-operative treatment, surgery can be less invasive (e.g. TEM surgery) or can even be omitted in selected patients (the wait and see approach).

The challenge for the future will be an early identification of different biological groups within all rectal cancers. Clinically, four groups of tumors can be identified:

1. Early tumors: small $(<3 \mathrm{~cm})$, clinically node negative tumors

2. Tumors growing mainly locoregionally, responding very well to neoadjuvant treatment

3. Tumors growing mainly locoregionally, responding poorly to neoadjuvant treatment

4. Tumors with a strong propensity to metastasize rapidly, often responding minimally to local treatment

TME surgery will be overtreatment in the majority of patients from the first group (early tumors). A local treatment, e.g. TEM surgery [94], brachytherapy or contact radiotherapy [95-97] can be an organ sparing alternative in the case of negative lymph nodes. At present, TEM surgery is an accepted alternative for T1, grade 1-2 tumors without angioinvasion. The difficulty in clinical practice is the prediction of nodal status. With standard imaging techniques, this prediction is notoriously unreliable $[98,99]$. Rapid developments in imaging techniques help to improve the predictive value, but rapid implementation of these techniques in clinical practice is hampered by the high technical demands and the fact that interpretation of these images is time consuming. Computer aided techniques 
could be helpful in the future to solve the problem of increased workload and to diminish inter-observer variability $[100,101]$.

For the second group (locoregional growth, responding well to (chemo)radiation), an intensification of locoregional treatment is an attractive approach. Further studies are needed to identify the optimal strategy for treatment intensification in these patients, e.g. an increase of radiotherapy dose or a combination with different radiosensitizers. Maybe for this biologically favorable group, increasing the radiation dose can already result in a substantial increase in complete responses, opening the way to organ preservation.

The third group (poor response to chemoradiation) is expected to be more heterogeneous biologically. Just as for the second group, treatment intensification may lead to more complete responders. The difficulty will be that a single strategy will not fit all tumors in this group. Therefore, better predictive models are needed to stratify patients into different treatment regimens. Ideally, these models should incorporate information about different aspects of tumor biology: not only clinical information, but also imaging data, genetic alterations and blood biomarkers for example. It may finally turn out that a "pan-omics" approach will help us with the most optimal treatment choice [102]. However, it is important to keep in mind that more complete responses do not necessarily translate into better outcome. The biggest gain of developing a $\mathrm{pCR}$, is the possibility of organ sparing treatment. It has still to be proven, however, that this approach is equivalent to standard neoadjuvant treatment and surgery in terms of oncological outcome.

The fourth group (rapid development of metastases) consists of tumors with a very aggressive biology. In these tumors intensification of local treatment is unlikely to result in better outcome. Short course radiotherapy followed by aggressive systemic therapy may be the most optimal approach in this patient group. It has already been shown in the metastasized setting that this treatment leads to a high rate of good local response [103]. 


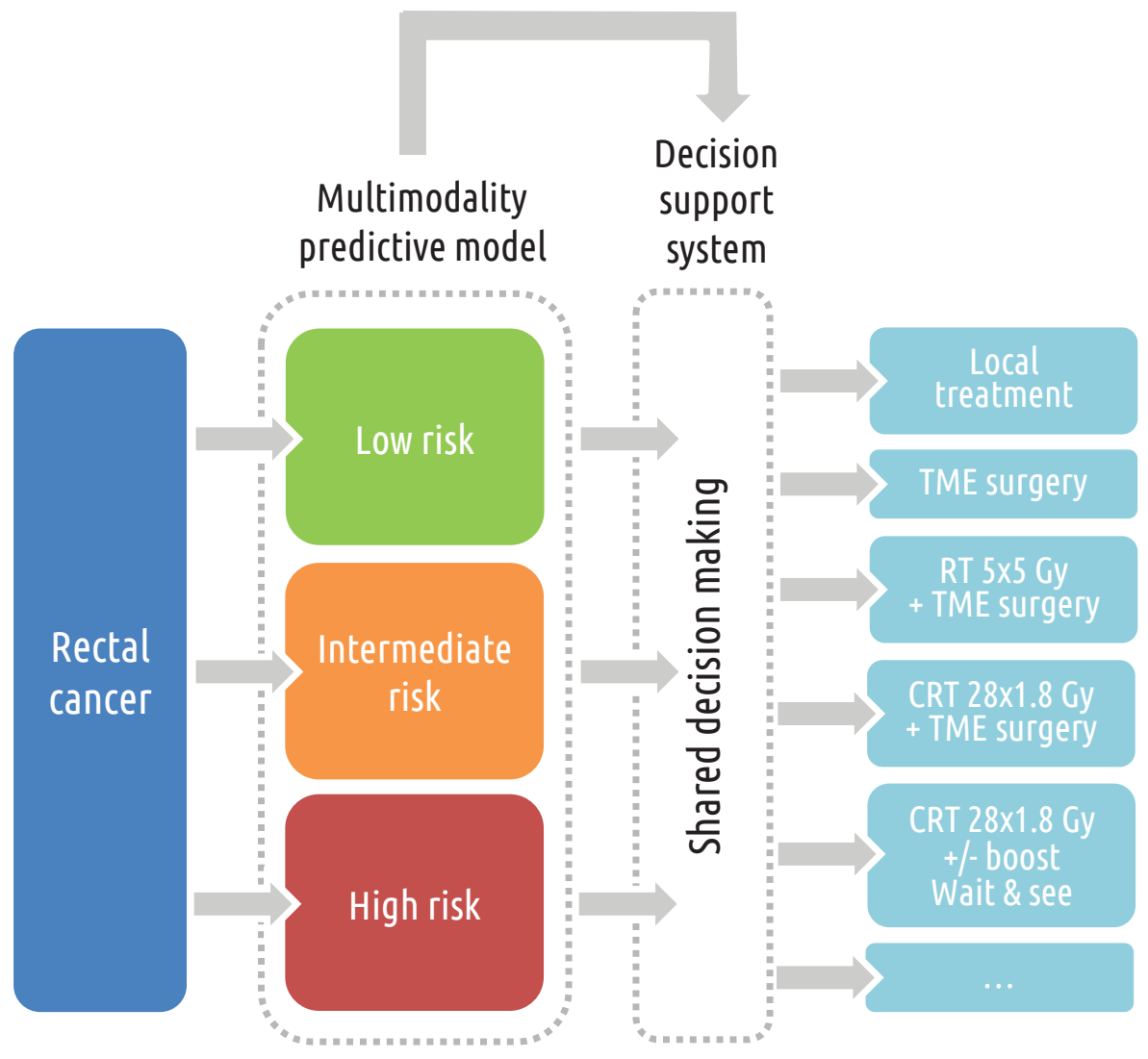

Figure 2 Possible treatment algorithm incorporating predictive models and shared decision making, leading to better patient tailored treatment.

The considerations for future developments in rectal cancer treatment mentioned above, all focus on the best outcome in terms of recurrence free and overall survival and do not take the functional outcome of the different treatment strategies into account. It is known from studies in prostate cancer that patients do not always choose the treatment with the highest chance of cure, if this more aggressive treatment has a higher chance of long term toxicity [104]. We therefore need to incorporate patient's preferences in the final treatment decision. However, this is only possible if patients are well informed about the benefits and harms of each treatment. Information given by 
physicians about long term side effects is known to be very variable [105]. Decision aids are helpful in guiding patients and doctors through the complex process of shared decision making [106]. Thus, decision aids should be developed for rectal cancer and these should ideally be combined with accurate predictive models. This opens the way to real patient-oriented tailored treatment (figure 2). 


\section{References}

[1] www.cijfersoverkanker.nl.

[2] Kapiteijn E, Marijnen CA, Nagtegaal ID, Putter H, Steup WH, Wiggers T, et al. Preoperative radiotherapy combined with total mesorectal excision for resectable rectal cancer. N Engl J Med. 2001;345:638-46.

[3] Sauer R, Becker H, Hohenberger W, Rodel C, Wittekind C, Fietkau R, et al. Preoperative versus postoperative chemoradiotherapy for rectal cancer. N Engl J Med. 2004;351:1731-40.

[4] Sebag-Montefiore D, Stephens RJ, Steele R, Monson J, Grieve R, Khanna S, et al. Preoperative radiotherapy versus selective postoperative chemoradiotherapy in patients with rectal cancer (MRC CR07 and NCIC-CTG C016): a multicentre, randomised trial. Lancet. 2009;373:811-20.

[5] van Gijn W, Marijnen CA, Nagtegaal ID, Kranenbarg EM, Putter H, Wiggers T, et al. Preoperative radiotherapy combined with total mesorectal excision for resectable rectal cancer: 12-year follow-up of the multicentre, randomised controlled TME trial. Lancet Oncol. 2011;12:575-82.

[6] Lange MM, den Dulk M, Bossema ER, Maas CP, Peeters KC, Rutten HJ, et al. Risk factors for faecal incontinence after rectal cancer treatment. Br J Surg. 2007;94:1278-84.

[7] Wiltink LM, Chen TY, Nout RA, Kranenbarg EM, Fiocco M, Laurberg S, et al. Health-related quality of life 14 years after preoperative short-term radiotherapy and total mesorectal excision for rectal cancer: report of a multicenter randomised trial. Eur J Cancer. 2014;50:2390-8.

[8] Habr-Gama A, Perez RO, Wynn G, Marks J, Kessler H, Gama-Rodrigues J. Complete clinical response after neoadjuvant chemoradiation therapy for distal rectal cancer: characterization of clinical and endoscopic findings for standardization. Dis Colon Rectum. 2010;53:1692-8.

[9] Maas M, Beets-Tan RG, Lambregts DM, Lammering G, Nelemans PJ, Engelen SM, et al. Waitand-see policy for clinical complete responders after chemoradiation for rectal cancer. J Clin Oncol. 2011;29:4633-40.

[10] Glynne-Jones R, Hughes R. Critical appraisal of the 'wait and see' approach in rectal cancer for clinical complete responders after chemoradiation. Br J Surg. 2012;99:897-909.

[11] Park IJ, You YN, Skibber JM, Rodriguez-Bigas MA, Feig B, Nguyen S, et al. Comparative analysis of lymph node metastases in patients with ypT0-2 rectal cancers after neoadjuvant chemoradiotherapy. Dis Colon Rectum. 2013;56:135-41.

[12] Pucciarelli S, Capirci C, Emanuele U, Toppan P, Friso ML, Pennelli GM, et al. Relationship between pathologic T-stage and nodal metastasis after preoperative chemoradiotherapy for locally advanced rectal cancer. Ann Surg Oncol. 2005;12:111-6.

[13] Read TE, Andujar JE, Caushaj PF, Johnston DR, Dietz DW, Myerson RJ, et al. Neoadjuvant therapy for rectal cancer: histologic response of the primary tumor predicts nodal status. Dis 
Colon Rectum. 2004;47:825-31.

[14] van Gijn W, Brehm V, de Graaf E, Neijenhuis PA, Stassen LP, Leijtens JW, et al. Unexpected rectal cancer after TEM: outcome of completion surgery compared with primary TME. Eur J Surg Oncol. 2013;39:1225-9.

[15] Bach SP, Hill J, Monson JR, Simson JN, Lane L, Merrie A, et al. A predictive model for local recurrence after transanal endoscopic microsurgery for rectal cancer. Br J Surg. 2009;96:280-90.

[16] Borschitz T, Heintz A, Junginger T. The influence of histopathologic criteria on the long-term prognosis of locally excised pT1 rectal carcinomas: results of local excision (transanal endoscopic microsurgery) and immediate reoperation. Dis Colon Rectum. 2006;49:1492-506; discussion 500-5.

[17] Bujko K, Richter P, Smith FM, Polkowski W, Szczepkowski M, Rutkowski A, et al. Preoperative radiotherapy and local excision of rectal cancer with immediate radical re-operation for poor responders: a prospective multicentre study. Radiother Oncol. 2013;106:198-205.

[18] Gagliardi G, Newton TR, Bailey HR. Local excision of rectal cancer followed by radical surgery because of poor prognostic features does not compromise the long term oncologic outcome. Colorectal Dis. 2013;15:e659-64.

[19] Lezoche E, Baldarelli M, Lezoche G, Paganini AM, Gesuita R, Guerrieri M. Randomized clinical trial of endoluminal locoregional resection versus laparoscopic total mesorectal excision for T2 rectal cancer after neoadjuvant therapy. Br J Surg. 2012;99:1211-8.

[20] Pucciarelli S, De Paoli A, Guerrieri M, La Torre G, Maretto I, De Marchi F, et al. Local excision after preoperative chemoradiotherapy for rectal cancer: results of a multicenter phase II clinical trial. Dis Colon Rectum. 2013;56:1349-56.

[21] Habr-Gama A, Gama-Rodrigues J, Sao Juliao GP, Proscurshim I, Sabbagh C, Lynn PB, et al. Local recurrence after complete clinical response and watch and wait in rectal cancer after neoadjuvant chemoradiation: impact of salvage therapy on local disease control. Int J Radiat Oncol Biol Phys. 2014;88:822-8.

[22] Fuss M. Strategies of assessing and quantifying radiation treatment metabolic tumor response using F18 FDG Positron Emission Tomography (PET). Acta Oncol. 2010;49:948-55.

[23] Lammering G, De Ruysscher D, van Baardwijk A, Baumert BG, Borger J, Lutgens L, et al. The use of FDG-PET to target tumors by radiotherapy. Strahlenther Onkol. 2010;186:471-81.

[24] Troost EG, Schinagl DA, Bussink J, Oyen WJ, Kaanders JH. Clinical evidence on PET-CT for radiation therapy planning in head and neck tumors. Radiother Oncol. 2010;96:328-34.

[25] De Ruysscher D, Nestle U, Jeraj R, Macmanus M. PET scans in radiotherapy planning of lung cancer. Lung Cancer. 2012;75:141-5.

[26] van Baardwijk A, Bosmans G, Boersma L, Buijsen J, Wanders S, Hochstenbag M, et al. PET-CTbased auto-contouring in non-small-cell lung cancer correlates with pathology and reduces interobserver variability in the delineation of the primary tumor and involved nodal volumes. Int J Radiat Oncol Biol Phys. 2007;68:771-8. 
[27] Daisne JF, Duprez T, Weynand B, Lonneux M, Hamoir M, Reychler H, et al. Tumor volume in pharyngolaryngeal squamous cell carcinoma: comparison at CT, MR imaging, and FDG PET and validation with surgical specimen. Radiology. 2004;233:93-100.

[28] Prieto E, Lecumberri P, Pagola M, Gomez M, Bilbao I, Ecay M, et al. Twelve automated thresholding methods for segmentation of PET images: a phantom study. Phys Med Biol. 2012;57:3963-80.

[29] Hatt M, Cheze le Rest C, Descourt P, Dekker A, De Ruysscher D, Oellers M, et al. Accurate automatic delineation of heterogeneous functional volumes in positron emission tomography for oncology applications. Int J Radiat Oncol Biol Phys. 2010;77:301-8.

[30] Withofs N, Bernard C, Van der Rest C, Martinive P, Hatt M, Jodogne S, et al. FDG PET/CT for rectal carcinoma radiotherapy treatment planning: comparison of functional volume delineation algorithms and clinical challenges. J Appl Clin Med Phys. 2014;15:4696.

[31] Hatt M, Cheze-Le Rest C, Aboagye EO, Kenny LM, Rosso L, Turkheimer FE, et al. Reproducibility of 18F-FDG and 3'-deoxy-3'-18F-fluorothymidine PET tumor volume measurements. J Nucl Med. 2010;51:1368-76.

[32] Gwynne S, Webster R, Adams R, Mukherjee S, Coles B, Staffurth J. Image-guided radiotherapy for rectal cancer: a systematic review. Clin Oncol (R Coll Radiol). 2012;24:250-60.

[33] Li C, Lan X, Yuan H, Feng H, Xia X, Zhang Y. 18F-FDG PET predicts pathological response to preoperative chemoradiotherapy in patients with primary rectal cancer: a meta-analysis. Ann Nucl Med. 2014;28:436-46.

[34] Capirci C, Rampin L, Erba PA, Galeotti F, Crepaldi G, Banti E, et al. Sequential FDG-PET/CT reliably predicts response of locally advanced rectal cancer to neo-adjuvant chemo-radiation therapy. Eur J Nucl Med Mol Imaging. 2007;34:1583-93.

[35] Denecke T, Rau B, Hoffmann KT, Hildebrandt B, Ruf J, Gutberlet M, et al. Comparison of CT, MRI and FDG-PET in response prediction of patients with locally advanced rectal cancer after multimodal preoperative therapy: is there a benefit in using functional imaging? Eur Radiol. 2005;15:1658-66.

[36] Konski A, Li T, Sigurdson E, Cohen SJ, Small W, Jr., Spies S, et al. Use of molecular imaging to predict clinical outcome in patients with rectal cancer after preoperative chemotherapy and radiation. Int J Radiat Oncol Biol Phys. 2009;74:55-9.

[37] Beets-Tan RG, Beets GL. MRI for assessing and predicting response to neoadjuvant treatment in rectal cancer. Nat Rev Gastroenterol Hepatol. 2014;11:480-8.

[38] Intven M, Reerink O, Philippens ME. Diffusion-weighted MRI in locally advanced rectal cancer : pathological response prediction after neo-adjuvant radiochemotherapy. Strahlenther Onkol. 2013;189:117-22.

[39] Lambrecht M, Vandecaveye V, De Keyzer F, Roels S, Penninckx F, Van Cutsem E, et al. Value of diffusion-weighted magnetic resonance imaging for prediction and early assessment of response to neoadjuvant radiochemotherapy in rectal cancer: preliminary results. Int $J$ 
Radiat Oncol Biol Phys. 2012;82:863-70.

[40] Monguzzi L, Ippolito D, Bernasconi DP, Trattenero C, Galimberti S, Sironi S. Locally advanced rectal cancer: value of $A D C$ mapping in prediction of tumor response to radiochemotherapy. Eur J Radiol. 2013;82:234-40.

[41] Sassen S, de Booij M, Sosef M, Berendsen R, Lammering G, Clarijs R, et al. Locally advanced rectal cancer: is diffusion weighted MRI helpful for the identification of complete responders (ypTON0) after neoadjuvant chemoradiation therapy? Eur Radiol. 2013;23:3440-9.

[42] Alberda WJ, Dassen HP, Dwarkasing RS, Willemssen FE, van der Pool AE, de Wilt JH, et al. Prediction of tumor stage and lymph node involvement with dynamic contrast-enhanced MRI after chemoradiotherapy for locally advanced rectal cancer. Int J Colorectal Dis. 2013;28:57380.

[43] Intven M, Reerink O, Philippens ME. Dynamic contrast enhanced MR imaging for rectal cancer response assessment after neo-adjuvant chemoradiation. J Magn Reson Imaging. 2014.

[44] Lambrecht M, Deroose C, Roels S, Vandecaveye V, Penninckx F, Sagaert X, et al. The use of FDG-PET/CT and diffusion-weighted magnetic resonance imaging for response prediction before, during and after preoperative chemoradiotherapy for rectal cancer. Acta Oncol. 2010;49:956-63.

[45] Das P, Skibber JM, Rodriguez-Bigas MA, Feig BW, Chang GJ, Wolff RA, et al. Predictors of tumor response and downstaging in patients who receive preoperative chemoradiation for rectal cancer. Cancer. 2007;109:1750-5.

[46] Park JW, Lim SB, Kim DY, Jung KH, Hong YS, Chang HJ, et al. Carcinoembryonic antigen as a predictor of pathologic response and a prognostic factor in locally advanced rectal cancer patients treated with preoperative chemoradiotherapy and surgery. Int J Radiat Oncol Biol Phys. 2009;74:810-7.

[47] Yoon SM, Kim DY, Kim TH, Jung KH, Chang HJ, Koom WS, et al. Clinical parameters predicting pathologic tumor response after preoperative chemoradiotherapy for rectal cancer. Int J Radiat Oncol Biol Phys. 2007;69:1167-72.

[48] Hotchi M, Shimada M, Kurita N, Iwata T, Sato H, Morimoto S, et al. microRNA expression is able to predict response to chemoradiotherapy in rectal cancer. Mol Clin Oncol. 2013;1:137-42.

[49] Kim JC, Ha YJ, Roh SA, Cho DH, Choi EY, Kim TW, et al. Novel single-nucleotide polymorphism markers predictive of pathologic response to preoperative chemoradiation therapy in rectal cancer patients. Int J Radiat Oncol Biol Phys. 2013;86:350-7.

[50] Watanabe T, Kobunai T, Akiyoshi T, Matsuda K, Ishihara S, Nozawa K. Prediction of response to preoperative chemoradiotherapy in rectal cancer by using reverse transcriptase polymerase chain reaction analysis of four genes. Dis Colon Rectum. 2014;57:23-31.

[51] Palma P, Cano C, Conde-Muino R, Comino A, Bueno P, Ferron JA, et al. Expression profiling of rectal tumors defines response to neoadjuvant treatment related genes. PLoS One. 2014;9:e112189. 
[52] Boulis-Wassif S, Gerard A, Loygue J, Camelot D, Buyse M, Duez N. Final results of a randomized trial on the treatment of rectal cancer with preoperative radiotherapy alone or in combination with 5-fluorouracil, followed by radical surgery. Trial of the European Organization on Research and Treatment of Cancer Gastrointestinal Tract Cancer Cooperative Group. Cancer. 1984;53:1811-8.

[53] De Caluwe L, Van Nieuwenhove Y, Ceelen WP. Preoperative chemoradiation versus radiation alone for stage II and III resectable rectal cancer. Cochrane Database Syst Rev. 2013;2:CD006041.

[54] Rodel C, Liersch T, Becker H, Fietkau R, Hohenberger W, Hothorn T, et al. Preoperative chemoradiotherapy and postoperative chemotherapy with fluorouracil and oxaliplatin versus fluorouracil alone in locally advanced rectal cancer: initial results of the German CAO/ARO/AIO-04 randomised phase 3 trial. Lancet Oncol. 2012;13:679-87.

[55] Aschele C, Cionini L, Lonardi S, Pinto C, Cordio S, Rosati G, et al. Primary tumor response to preoperative chemoradiation with or without oxaliplatin in locally advanced rectal cancer: pathologic results of the STAR-01 randomized phase III trial. J Clin Oncol. 2011;29:2773-80.

[56] Gerard JP, Azria D, Gourgou-Bourgade S, Martel-Lafay I, Hennequin C, Etienne PL, et al. Clinical outcome of the ACCORD 12/0405 PRODIGE 2 randomized trial in rectal cancer. J Clin Oncol. 2012;30:4558-65.

[57] Klautke G, Feyerherd P, Ludwig K, Prall F, Foitzik T, Fietkau R. Intensified concurrent chemoradiotherapy with 5-fluorouracil and irinotecan as neoadjuvant treatment in patients with locally advanced rectal cancer. Br J Cancer. 2005;92:1215-20.

[58] Mohiuddin M, Winter K, Mitchell E, Hanna N, Yuen A, Nichols C, et al. Randomized phase II study of neoadjuvant combined-modality chemoradiation for distal rectal cancer: Radiation Therapy Oncology Group Trial 0012. J Clin Oncol. 2006;24:650-5.

[59] Navarro M, Dotor E, Rivera F, Sanchez-Rovira P, Vega-Villegas ME, Cervantes A, et al. A Phase II study of preoperative radiotherapy and concomitant weekly irinotecan in combination with protracted venous infusion 5-fluorouracil, for resectable locally advanced rectal cancer. Int J Radiat Oncol Biol Phys. 2006;66:201-5.

[60] Sato T, Ozawa H, Hatate K, Onosato W, Naito M, Nakamura T, et al. A Phase II trial of neoadjuvant preoperative chemoradiotherapy with S-1 plus irinotecan and radiation in patients with locally advanced rectal cancer: clinical feasibility and response rate. Int $\mathrm{J}$ Radiat Oncol Biol Phys. 2011;79:677-83.

[61] Shin SJ, Kim NK, Keum KC, Kim HG, Im JS, Choi HJ, et al. Phase II study of preoperative chemoradiotherapy (CRT) with irinotecan plus S-1 in locally advanced rectal cancer. Radiother Oncol. 2010;95:303-7.

[62] Wong SJ, Winter K, Meropol NJ, Anne PR, Kachnic L, Rashid A, et al. Radiation Therapy Oncology Group 0247: a randomized Phase II study of neoadjuvant capecitabine and irinotecan or capecitabine and oxaliplatin with concurrent radiotherapy for patients with 
locally advanced rectal cancer. Int J Radiat Oncol Biol Phys. 2012;82:1367-75.

[63] Dahabreh IJ, Terasawa T, Castaldi PJ, Trikalinos TA. Systematic review: Anti-epidermal growth factor receptor treatment effect modification by KRAS mutations in advanced colorectal cancer. Ann Intern Med. 2011;154:37-49.

[64] Bonner JA, Harari PM, Giralt J, Azarnia N, Shin DM, Cohen RB, et al. Radiotherapy plus cetuximab for squamous-cell carcinoma of the head and neck. N Engl J Med. 2006;354:56778.

[65] Dewdney A, Cunningham D, Tabernero J, Capdevila J, Glimelius B, Cervantes A, et al. Multicenter randomized phase II clinical trial comparing neoadjuvant oxaliplatin, capecitabine, and preoperative radiotherapy with or without cetuximab followed by total mesorectal excision in patients with high-risk rectal cancer (EXPERT-C). J Clin Oncol. 2012;30:1620-7.

[66] Fokas E, Conradi L, Weiss C, Sprenger T, Middel P, Rau T, et al. Preoperative chemoradiation therapy with capecitabine/oxaliplatin and cetuximab in rectal cancer: long-term results of a prospective phase 1/2 study. Int J Radiat Oncol Biol Phys. 2013;87:992-9.

[67] Horisberger K, Treschl A, Mai S, Barreto-Miranda M, Kienle P, Strobel P, et al. Cetuximab in combination with capecitabine, irinotecan, and radiotherapy for patients with locally advanced rectal cancer: results of a Phase II MARGIT trial. Int J Radiat Oncol Biol Phys. 2009;74:1487-93.

[68] Kim SY, Hong YS, Kim DY, Kim TW, Kim JH, Im SA, et al. Preoperative chemoradiation with cetuximab, irinotecan, and capecitabine in patients with locally advanced resectable rectal cancer: a multicenter Phase II study. Int J Radiat Oncol Biol Phys. 2011;81:677-83.

[69] Machiels JP, Sempoux C, Scalliet P, Coche JC, Humblet Y, Van Cutsem E, et al. Phase I/II study of preoperative cetuximab, capecitabine, and external beam radiotherapy in patients with rectal cancer. Ann Oncol. 2007;18:738-44.

[70] Sun PL, Li B, Ye QF. Effect of neoadjuvant cetuximab, capecitabine, and radiotherapy for locally advanced rectal cancer: results of a phase II study. Int J Colorectal Dis. 2012;27:1325-32.

[71] Velenik V, Ocvirk J, Oblak I, Anderluh F. Cetuximab in preoperative treatment of rectal cancer - term outcome of the XERT trial. Radiol Oncol. 2012;46:252-7.

[72] Grimminger PP, Danenberg P, Dellas K, Arnold D, Rodel C, Machiels JP, et al. Biomarkers for cetuximab-based neoadjuvant radiochemotherapy in locally advanced rectal cancer. Clin Cancer Res. 2011;17:3469-77.

[73] Nyati MK, Morgan MA, Feng FY, Lawrence TS. Integration of EGFR inhibitors with radiochemotherapy. Nat Rev Cancer. 2006;6:876-85.

[74] Lawrence TS, Blackstock AW, McGinn C. The mechanism of action of radiosensitization of conventional chemotherapeutic agents. Semin Radiat Oncol. 2003;13:13-21.

[75] Crane CH, Eng C, Feig BW, Das P, Skibber JM, Chang GJ, et al. Phase II trial of neoadjuvant bevacizumab, capecitabine, and radiotherapy for locally advanced rectal cancer. Int J Radiat 
Oncol Biol Phys. 2010;76:824-30.

[76] Czito BG, Bendell JC, Willett CG, Morse MA, Blobe GC, Tyler DS, et al. Bevacizumab, oxaliplatin, and capecitabine with radiation therapy in rectal cancer: Phase I trial results. Int J Radiat Oncol Biol Phys. 2007;68:472-8.

[77] Dellas K, Hohler T, Reese T, Wurschmidt F, Engel E, Rodel C, et al. Phase II trial of preoperative radiochemotherapy with concurrent bevacizumab, capecitabine and oxaliplatin in patients with locally advanced rectal cancer. Radiat Oncol. 2013;8:90.

[78] Dipetrillo T, Pricolo V, Lagares-Garcia J, Vrees M, Klipfel A, Cataldo T, et al. Neoadjuvant bevacizumab, oxaliplatin, 5-fluorouracil, and radiation for rectal cancer. Int J Radiat Oncol Biol Phys. 2012;82:124-9.

[79] Gasparini G, Torino F, Ueno T, Cascinu S, Troiani T, Ballestrero A, et al. A phase II study of neoadjuvant bevacizumab plus capecitabine and concomitant radiotherapy in patients with locally advanced rectal cancer. Angiogenesis. 2012;15:141-50.

[80] Kennecke H, Berry S, Wong R, Zhou C, Tankel K, Easaw J, et al. Pre-operative bevacizumab, capecitabine, oxaliplatin and radiation among patients with locally advanced or low rectal cancer: a phase II trial. Eur J Cancer. 2012;48:37-45.

[81] Koukourakis MI, Giatromanolaki A, Tsoutsou P, Lyratzopoulos N, Pitiakoudis M, Kouklakis G, et al. Bevacizumab, capecitabine, amifostine, and preoperative hypofractionated accelerated radiotherapy (HypoArc) for rectal cancer: a Phase II study. Int J Radiat Oncol Biol Phys. 2011;80:492-8.

[82] Landry JC, Feng Y, Cohen SJ, Staley CA, 3rd, Whittington R, Sigurdson ER, et al. Phase 2 study of preoperative radiation with concurrent capecitabine, oxaliplatin, and bevacizumab followed by surgery and postoperative 5-fluorouracil, leucovorin, oxaliplatin (FOLFOX), and bevacizumab in patients with locally advanced rectal cancer: ECOG 3204. Cancer. 2013;119:1521-7.

[83] Liang JT, Lai HS, Cheng KW. Technical feasibility of laparoscopic total mesorectal excision for patients with low rectal cancer after concurrent radiation and chemotherapy with bevacizumab plus FOLFOX. Surg Endosc. 2011;25:305-8.

[84] Nogue M, Salud A, Vicente P, Arrivi A, Roca JM, Losa F, et al. Addition of bevacizumab to XELOX induction therapy plus concomitant capecitabine-based chemoradiotherapy in magnetic resonance imaging-defined poor-prognosis locally advanced rectal cancer: the AVACROSS study. Oncologist. 2011;16:614-20.

[85] Resch G, De Vries A, Ofner D, Eisterer W, Rabl H, Jagoditsch M, et al. Preoperative treatment with capecitabine, bevacizumab and radiotherapy for primary locally advanced rectal cancer--a two stage phase II clinical trial. Radiother Oncol. 2012;102:10-3.

[86] Spigel DR, Bendell JC, McCleod M, Shipley DL, Arrowsmith E, Barnes EK, et al. Phase II study of bevacizumab and chemoradiation in the preoperative or adjuvant treatment of patients with stage II/III rectal cancer. Clin Colorectal Cancer. 2012;11:45-52. 
[87] Velenik V, Ocvirk J, Music M, Bracko M, Anderluh F, Oblak I, et al. Neoadjuvant capecitabine, radiotherapy, and bevacizumab (CRAB) in locally advanced rectal cancer: results of an openlabel phase II study. Radiat Oncol. 2011;6:105.

[88] Willett CG, Duda DG, Ancukiewicz M, Shah M, Czito BG, Bentley R, et al. A safety and survival analysis of neoadjuvant bevacizumab with standard chemoradiation in a phase $\mathrm{I} / \mathrm{Il}$ study compared with standard chemoradiation in locally advanced rectal cancer. Oncologist. 2010;15:845-51.

[89] Simon R. Lost in translation: problems and pitfalls in translating laboratory observations to clinical utility. Eur J Cancer. 2008;44:2707-13.

[90] Fruman DA, Rommel C. PI3K and cancer: lessons, challenges and opportunities. Nat Rev Drug Discov. 2014;13:140-56.

[91] Laplante M, Sabatini DM. mTOR signaling in growth control and disease. Cell. 2012;149:27493.

[92] Manegold PC, Paringer C, Kulka U, Krimmel K, Eichhorn ME, Wilkowski R, et al. Antiangiogenic therapy with mammalian target of rapamycin inhibitor RAD001 (Everolimus) increases radiosensitivity in solid cancer. Clin Cancer Res. 2008;14:892-900.

[93] Myers AL, Orr WS, Denbo JW, Ng CY, Zhou J, Spence Y, et al. Rapamycin-induced tumor vasculature remodeling in rhabdomyosarcoma xenografts increases the effectiveness of adjuvant ionizing radiation. J Pediatr Surg. 2012;47:183-9.

[94] Winde G, Nottberg H, Keller R, Schmid KW, Bunte H. Surgical cure for early rectal carcinomas (T1). Transanal endoscopic microsurgery vs. anterior resection. Dis Colon Rectum. 1996;39:969-76.

[95] Coatmeur O, Truc G, Barillot I, Horiot JC, Maingon P. Treatment of T1-T2 rectal tumors by contact therapy and interstitial brachytherapy. Radiother Oncol. 2004;70:177-82.

[96] Marijnen CA. External beam radiotherapy and high dose rate brachytherapy for medically unfit and elderly patients. Clin Oncol (R Coll Radiol). 2007;19:706-10.

[97] Christoforidis D, McNally MP, Jarosek SL, Madoff RD, Finne CO. Endocavitary contact radiation therapy for ultrasonographically staged T1 NO and T2 NO rectal cancer. Br J Surg. 2009;96:430-6.

[98] Bipat S, Glas AS, Slors FJ, Zwinderman AH, Bossuyt PM, Stoker J. Rectal cancer: local staging and assessment of lymph node involvement with endoluminal US, CT, and MR imaging--a meta-analysis. Radiology. 2004;232:773-83.

[99] Lahaye MJ, Engelen SM, Nelemans PJ, Beets GL, van de Velde CJ, van Engelshoven JM, et al. Imaging for predicting the risk factors--the circumferential resection margin and nodal disease--of local recurrence in rectal cancer: a meta-analysis. Semin Ultrasound CT MR. 2005;26:259-68.

[100] Kumar V, Gu Y, Basu S, Berglund A, Eschrich SA, Schabath MB, et al. Radiomics: the process and the challenges. Magn Reson Imaging. 2012;30:1234-48. 
[101] Lambin P, Rios-Velazquez E, Leijenaar R, Carvalho S, van Stiphout RG, Granton P, et al. Radiomics: extracting more information from medical images using advanced feature analysis. Eur J Cancer. 2012;48:441-6.

[102] Shrager J, Tenenbaum JM. Rapid learning for precision oncology. Nat Rev Clin Oncol. 2014;11:109-18.

[103] van Dijk TH, Tamas K, Beukema JC, Beets GL, Gelderblom AJ, de Jong KP, et al. Evaluation of short-course radiotherapy followed by neoadjuvant bevacizumab, capecitabine, and oxaliplatin and subsequent radical surgical treatment in primary stage IV rectal cancer. Ann Oncol. 2013;24:1762-9.

[104] van Tol-Geerdink JJ, Stalmeier PF, van Lin EN, Schimmel EC, Huizenga H, van Daal WA, et al. Do patients with localized prostate cancer treatment really want more aggressive treatment? J Clin Oncol. 2006;24:4581-6.

[105] Kunneman M, Marijnen CA, Rozema T, Ceha HM, Grootenboers DA, Neelis KJ, et al. Decision consultations on preoperative radiotherapy for rectal cancer: large variation in benefits and harms that are addressed. Br J Cancer. 2014.

[106] van Tol-Geerdink JJ, Willem Leer J, Weijerman PC, van Oort IM, Vergunst H, van Lin EN, et al. Choice between prostatectomy and radiotherapy when men are eligible for both: a randomized controlled trial of usual care vs decision aid. BJU Int. 2013;111:564-73. 
Summary and valorization

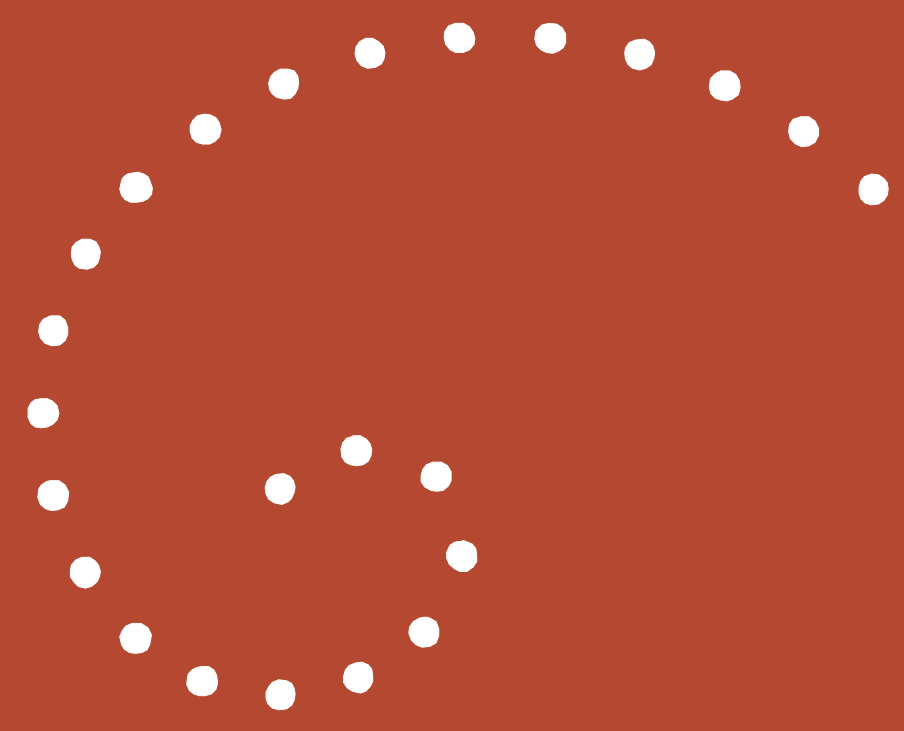



Chapter 10

Summary 
In this thesis three different strategies that can lead to treatment individualization and optimization in rectal cancer are discussed. In the first part, the possible role of PET-CT in radiotherapy treatment planning for rectal tumors is investigated. Accurate tumor delineation is necessary to be able to implement boost techniques with the aim of dose escalation. The second part is devoted to response prediction in rectal cancer. Reliable response prediction is essential to tailor treatment and to give advice to patients about the optimal treatment. In the third part, two early phase clinical trials are presented testing the addition of an Akt inhibitor and an mTOR inhibitor to different neoadjuvant treatments in rectal cancer. The goal of these trials was to enhance tumor response to neo-adjuvant treatment.

\section{Part I: The role of PET-CT in tumor delineation}

In chapter 2, the correlation between tumor length measured in the pathology specimen and on different imaging modalities is investigated. Rectal tumors of 26 patients were measured manually on CT, MR and PET-scan. Furthermore an automated tumor length was derived from PET-scan, using the signal-tobackground (SBR) method. Measurements based on PET-scan correlated better with tumor length in the surgical specimen than those based on CT or MR and automated PET-measurements performed better than manual measurements.

Chapter 3 discusses the results of a delineation study incorporating different imaging modalities. Tumors of 42 rectal cancer patients were delineated by 5 observers 3 times: using only CT and MR, using CT, MR and PET and using all three modalities including an automatic contour based on PET-scan. Tumor volumes delineated using PET information were significantly smaller than volumes determined on CT and MR and the smallest volumes were created using the automatic delineation method. With respect to interobserver variation, a significant increase of the conformity index was observed when PET-information was added to the delineation process, resulting in a mean conformity index of 0.93 using PET auto-contours. The caudal border of the treatment volume can be adapted using PET information. 


\section{Part II: Response prediction in rectal cancer treatment}

Prediction of pathological complete response (pCR) after chemoradiation (CRT) using sequential PET-imaging is described in chapter 4. Large databases of 4 different institutes were used to build a prediction model. A PET-scan was made before the start of treatment and approximately 8 weeks after the end of chemoradiation. The strongest model for prediction of $\mathrm{PCR}$ was based on posttreatment PET-results and included tumor length, post-treatment SUV $V_{\max }$ and relative change in SUV $V_{\max }$, resulting in an area under the ROC curve (AUC) of 0.83 for the validation set.

The value of blood biomarkers for response prediction after CRT was studied in chapter 5. Blood samples of 276 patients treated with CRT for locally advanced rectal cancer were analyzed for 9 serum biomarkers. The choice of these biomarkers was based on a pre-defined hypothesis. CEA turned out to be predictive for $\mathrm{PCR}$, while CEA and interleukin-8 (IL-8) predicted a good response (ypT0-2N0). The addition of biomarker information to an existing prediction model based on clinical data and PET-information led to an increase of the performance of these models, indicating that blood biomarkers have added value.

In chapter 6, the existing literature on biochemical and molecular biological factors and their role in decisions in the treatment of rectal cancer is reviewed. Until now, the value of this type of predictive factors turns out to be limited. Genetic biomarkers showing a predictive value for response to chemoradiation still need external validation and prospective evaluation in larger patient groups.

\section{Part III: Combined treatment in rectal cancer}

Nelfinavir, an inhibitor of the PI3K/Akt pathway, is a potential radiosensitizing drug. Its incorporation in a neoadjuvant chemoradiation schedule with capecitabine for rectal cancer treatment is tested in chapter 7 . Although the combination treatment was feasible, a high incidence of grade 3 toxicities was observed. Diarrhea and transaminase elevation were the most frequently occurring side effects, indicating possible overlapping toxicities between 
capecitabine and nelfinavir. The recommended dose for a phase II trial was 750 mg BID, the lowest dose level tested in this trial.

The inihibition of mTOR, another possible attractive target to increase radiosensitivity, is tested in chapter 8 . Rapamycin, an mTOR inhibitor, was added to 5x5 Gy pre-operative radiotherapy in a phase $1 / 1 \mathrm{l}$ trial. The combination turned out to be well tolerated, although a high number of postoperative complications was observed if the surgical resection was performed immediately after the end of radiotherapy and rapamycin. The primary endpoint, a decrease in tumor perfusion, was not reached. However, changes in metabolic activity reflected by changes in FDG-uptake on PET-scan, confirm the biological activity of rapamycin in rectal cancer. 
In dit proefschrift worden drie verschillende strategieën besproken die kunnen leiden tot de individualisering en optimalisering van de behandeling van het rectumcarcinoom. In deel I wordt de mogelijke rol van PET-CT in de radiotherapie planning voor rectumtumoren onderzocht. Om boost technieken te kunnen invoeren met het oog op dosis escalatie, is een betrouwbare intekening van de tumor onmisbaar. Deel II is gewijd aan respons predictie voor endeldarmkanker. Betrouwbare respons predictie is essentieel om behandeling op maat te kunnen bieden en om patiënten advies te kunnen geven over de optimale behandeling. In deel III worden twee klinische trials besproken die het toevoegen van een Akt-remmer en een mTOR-remmer aan twee verschillende neo-adjuvante behandelingen voor rectumcarcinoom onderzoeken. Het doel van deze behandelingen was het versterken van de respons van de tumoren op de neo-adjuvante behandeling.

\section{Deel I: De rol van PET-CT bij tumor delineatie}

In hoofdstuk 2 wordt de correlatie tussen tumorlengte gemeten in het pathologie preparaat en op de verschillende beeldvormingsmodaliteiten onderzocht. Rectumtumoren van 26 patiënten werden handmatig gemeten op CT, MRI en PET-scan. Verder werd een automatisch gemeten tumorlengte verkregen door het creëren van een automatische contour op de PET-scan met behulp van de source-to-background ratio (SBR) methode. Metingen gedaan op de PET-scan correleerden beter met de tumor lengte in het chirurgisch preparaat dan metingen op CT of MRI en de geautomatiseerde PET-metingen waren beter dan de handmatige metingen.

Hoofdstuk 3 behandelt de resultaten van een intekenstudie waarin verschillende beeldvormingsmodaliteiten worden gebruikt. Tumoren van 42 rectumcarcinoom patiënten werden 3 maal ingetekend door 5 verschillende observatoren: de eerste keer gebruik makend van CT en MRI, de tweede keer van CT, MRI en PET en de derde keer alle modaliteiten inclusief een automatisch gegenereerde contour gebaseerd op de PET-scan. De tumor volumes die werden ingetekend op basis van de PET-scan waren significant kleiner dan de 
volumes bepaald op grond van CT en MRI en de kleinste volumes werden verkregen met de automatische delineatie methode. Met betrekking tot de interobserver variatie werd een significante toename van de conformity index gezien als PET-informatie werd betrokken in het intekenproces, resulterend in een gemiddelde conformity index van 0,93 bij gebruik van automatische PETcontouren. De caudale begrenzing van het behandelvolume blijkt op grond van PET-informatie aangepast te kunnen worden.

\section{Deel II: Response predictie voor de behandeling van rectum- carcinoom}

In hoofdstuk 4 wordt de predictie van pathologisch complete respons (pCR) na chemoradiatie (CRT) met behulp van herhaalde PET-scans beschreven. Grote databases van 4 verschillende instituten werden gebruikt om een predictiemodel te bouwen. Voor de start van de behandeling en ongeveer 8 weken na het einde van de chemoradiatie werd een PET-scan gemaakt. Het sterkste predictiemodel voor pCR was gebaseerd op PET resultaten van na de behandeling en omvatte tumor lengte, de SUV $V_{\max }$ na de behandeling en de relatieve verandering in SUV $\mathrm{Sax}_{\text {m. }}$ Dit model resulteerde in een AUC van 0.83 voor de validatie set.

De waarde van bloed biomarkers voor respons predictie na CRT werd bestudeerd in hoofdstuk 5. Bloedmonsters van 276 patiënten behandeld met CRT voor lokaal uitgebreid rectumcarcinoom werden onderzocht op 9 serum biomarkers. De keuze voor de ze biomarkers was gebaseerd op een vooraf bepaalde hypothese. CEA bleek voorspellend te zijn voor pCR, terwijl CEA en interleukine-8 een goede respons (ypT0-2N0) konden voorspellen. De toevoeging van biomarker informatie aan een bestaand predictiemodel gebaseerd op klinische gegevens en PET-informatie leidde tot een sterkere predictie van deze modellen, hetgeen aantoont dat bloed biomarkers toegevoegde waarde heeft.

In hoofdstuk 6 wordt de literatuur over biochemische en moleculaire biologische factoren en hun rol in behandelbeslissingen voor het 
rectumcarcinoom besproken. Tot op heden blijkt de rol van dit type predictieve factoren beperkt te zijn. Genetische biomarkers die predictieve waarde voor de respons op chemoradiatie moeten nog extern gevalideerd en prospectief onderzocht worden in grotere groepen patiënten.

\section{Deel III: Combinatiebehandeling voor het rectumcarcinoom}

Nelfinavir, een remmer van de PI3K/Akt signaleringsroute, kan mogelijk de stralingsgevoeligheid van tumoren doen toenemen. In hoofdstuk 7 wordt de toevoeging van nelfinavir aan een neo-adjuvant chemoradiatie schema met capecitabine onderzocht. Hoewel de behandelcombinatie klinisch uitvoerbaar bleek te zijn, werd wel een hoge incidentie van graad 3 toxiciteit gezien. Diarree en transaminase stijgingen waren de meest frequent optredende bijwerkingen, duidend op mogelijke overlap van toxiciteit veroorzaakt door capecitabine en nelfinavir. De aanbevolen dosis voor een fase II trial was tweemaal daags $750 \mathrm{mg}$, het laagste dosisniveau dat werd onderzocht in deze trial.

De remming van mTOR, een ander mogelijk aantrekkelijk doelwit om de stralingsgevoeligheid verhogen, wordt beschreven in hoofdstuk 8. Rapamycin, een mTOR inhibitor, werd toegevoegd aan 5x5 Gy pre-operatieve radiotherapie in een fase I/II studie. De combinatie bleek goed verdragen te worden, hoewel er relatief veel postoperatieve complicaties werden gezien als de resectie direct na beëindiging van de radiotherapie met rapamycin werd uitgevoerd. Het primaire eindpunt, een afname in tumorperfusie, werd niet gehaald. Veranderingen in metabole activiteit, af te lezen uit verandering in FDG-opname op de PET-scan, bevestigen een biologische activiteit van rapamycin bij het rectumcarcinoom. 
Chapter 11

Valorization 


\section{Valorization}

Cancer is the leading cause of death in the Netherlands and its incidence is expected to increase substantially in the coming years. This expected rise in cancer incidence is even more pronounced due to the increasing proportion of elderly in the population and the increased life expectancy. Colorectal cancer is the second most occurring cancer type in men and the third in women. This makes rectal cancer an important health care problem.

Although the results of rectal cancer treatment have been improved substantially in the last decades, there is certainly room for improvement. Traditionally, cancer treatment has been based on clinical staging, which is a rather rough classification and results in an important percentage of under-and overtreatment. Rectal cancer treatment has been characterized by a tendency to overtreatment in the Netherlands in the last years, resulting in an increased risk of long-term sequelae and higher costs. Furthermore, it is expected that the distribution of clinical stages at the time of diagnosis will shift to earlier stages in the coming years, due to the recent introduction of a national screening program for bowel cancer. The most important themes in Dutch healthcare at this moment are control of costs, improvement of quality and shared decisionmaking. These considerations all ask for soundly based individualization of treatment.

The main theme of this thesis is the development of more tailored treatment of rectal cancer. This tailoring of treatment can consist of an intensification of treatment for patients who will benefit from it and a de-escalation of preoperative treatment in patients who have very sensitive tumors or who do not need neo-adjuvant treatment at all. To make it even more complex, treatment intensification can have two different aims: a better chance of locoregional control or a higher chance of complete response, opening the way to organ preservation. Although more information is needed about long-term outcome of oncological outcome of organ-preserving treatment, it has important advantages: post-operative complications are avoided, possible toxicity caused by the surgical intervention (fecal urge and incontinence, urinary incontinence, 
sexual dysfunction) does not occur and a colostomy can be avoided. This is expected to lead to less healthcare costs and a better quality of life. Therefore, if oncological safety of the watch-and-wait approach in clinical complete responders can be confirmed, it may become the ultimate goal of (chemo)radiotherapy in rectal cancer treatment.

\section{Relevance of the scientific results of this thesis}

The results presented in the first part of this thesis can be seen as a step in the development of boost techniques for radiotherapy in rectal cancer. Dose escalation is one possibility to increase the chance of a complete response in radiotherapy. The studies presented in this thesis have proven that PET-CT is a reliable and reproducible method for adequate delineation of the primary tumor, a prerequisite for tumor boosting.

The second part of this thesis presents the first steps in the development of predictive models for rectal cancer treatment. PET-scan has been proven to be a strong instrument in response prediction in rectal cancer treatment. The work presented here was a first indication that PET-information has predictive value. The nomogram has been made available online (www.predictcancer.org). Based on these results, we developed a program aimed at the optimal use of PET-scan in predictive models for rectal cancer. In the meantime, our group has published more articles about the use of PET for early response evaluation and we have found that PET-scan after 2 weeks of treatment is able to separate good responders from bad responders. This concept has been validated in a prospective multicenter trial.

The studies discussed in the third and last part were aimed at treatment intensification. Both studies are examples of early translation of laboratory findings into clinical practice. The second particularity of these 2 studies is the use of drugs that were registered already for other indications. The advantage of this approach is not only that the side effects of these drugs are well known, but also that the costs are substantially lower than the costs of recently developed and patented drugs. Unfortunately the combination of nelfinavir and 
chemoradiation turned out to have an unfavorable toxicity profile and the distribution of nelfinavir was stopped in the Netherlands, which made us decide not to go on to phase II. The combination of rapamycin and radiotherapy was well tolerated if surgery was delayed, but our hypothesis could not be confirmed. Although rapamycin had a clear biological activity in rectal cancer, as reflected by the SUV-changes on PET-scan, this did not translate into a substantial increase in response. Therefore, the scheme as tested in this phase $\mathrm{I} / \mathrm{II}$ trial is not promising enough to continue to a phase III trial.

\section{Innovation and future}

The principle of response prediction has been shown to be feasible in clinical practice. We therefore want to further explore the possibilities in clinical practice by adapting treatment based on early response prediction to increase the response rates. The use of prediction models allows us to intensify treatment only in patients who have a high chance to benefit from it, which is a new approach as compared to the "one-size-fits-all" recommendations in guidelines. Another important goal for future projects is to incorporate patient preferences into treatment decisions. This process will also be supported by reliable response prediction. 
Appendix

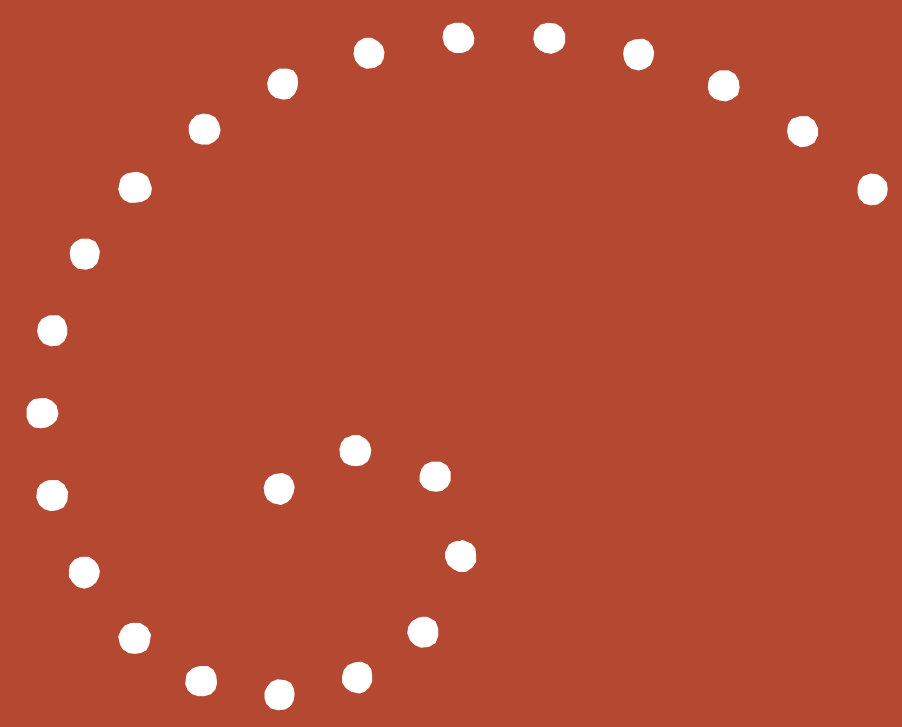



Appendix

Dankwoord 
$\mathrm{Na}$ al die jaren werken aan dit proefschrift rest het schrijven van het eerst gelezen hoofdstuk van ieder "boekje". Onderzoek doen is bij uitstek samenwerken en velen ben ik dan ook dank verschuldigd.

Laat ik beginnen met het bedanken van de mensen zonder wier bereidheid om extra onderzoeken te ondergaan, extra medicijnen te slikken en extra tijd te investeren, het doen van medisch wetenschappelijk onderzoek onmogelijk zou zijn: de patiënten. Geheel belangeloos hebben zij hun medewerking verleend om daarmee de behandeling van mensen die in de toekomst met endeldarmkanker geconfronteerd worden te verbeteren.

Beste Philippe, in het begin van mijn onderzoek zei je weleens "tu veux un pigeon rôti qui te vole dans la bouche". Jij kon daarbij niet weten dat ik zo'n hekel heb aan duiven dat ik ze maar op één plaats duld: op mijn bord. Jij was de afgelopen jaren echt de grote stimulator achter mijn onderzoek. Daar waar ik regelmatig obstakels zag, bleef jij onvermoeibaar met nieuwe ideeën komen.

Beste Guido, jij hebt me enorm geholpen met alle dagelijkse problemen die ik tegenkwam op onderzoeksgebied. Jaloers was ik altijd op de fraaie Engelse formuleringen die ik terug kreeg, als ik je een eerste versie van een artikel had gestuurd. Daarnaast heb ik veel van je geleerd op klinisch gebied, zodat ik het stokje van je kon overnemen toen je 2 jaar geleden terug ging naar Duitsland.

Beste Michel, dé vraagbaak voor alles wat met PET en CT te maken had. Altijd was jij bereid je input te geven vanuit de fysische achtergrond en jouw technische inbreng in het rectumgroepje was onmisbaar.

Ik wil graag de leden van de beoordelingscommissie (Prof. dr. F. Mottaghy, Dr. M. Lahaye, Prof. dr. C. Marijnen, Prof. dr. H. Rutte, Prof. dr. V. Tjan-Heijnen) bedanken voor hun tijd en energie om mijn proefschrift te beoordelen. 
Beste Marco en Ruud, dank voor de zeer prettige samenwerking. Ik heb het gevoel dat ik meer van jullie hulp heb geprofiteerd, dan andersom: ik hoefde maar bij jullie aan te kloppen of er werd weer een handig scriptje gemaakt of een mooie figuur geproduceerd. Zelfs toen jullie Maastro al verlaten hadden, kon ik nog op jullie hulp terug vallen.

Beste Jørgen, dank voor de vele uren die jij hebt besteed aan scans inlezen, structuren aanmaken, PET-scans analyseren en perfusiescans door pipelines halen. Ook nu blijft de samenwerking met "die dikke kale" (niet mijn woorden) gelukkig bestaan door je kartrekkersrol in de GE-zorglijn.

Alle collega chirurgen, internist-oncologen, pathologen, MDL-artsen en radiologen uit de regio: dank voor de uitermate prettige samenwerking. Ik heb het gevoel dat we de afgelopen jaren steeds beter gestroomlijnde zorg zijn gaan leveren aan onze gezamenlijke patiënten. Jullie bereidheid tot meewerken aan door Maastro geïnitieerde studies heeft geleid tot een deel van dit proefschrift.

Een speciaal woord van dank en bewondering voor het duo Beets/Beets-Tan. Beste Regina en Geerard, wat een voorrecht om met jullie team te werken. Je kunt je als radiotherapeut toch niet méér gewaardeerd voelen, dan door een chirurg die het aandurft patiënten niet te opereren na de bestraling. Erg jammer dat jullie het zuiden verlaten; heel veel succes bij jullie nieuwe uitdaging!

Alle Maastro collega's wil ik hartelijk danken voor de samenwerking. De sfeer binnen de afdeling, waarmee ik tijdens een keuze co-schap bij het RTIL kennis had gemaakt, is een van de redenen waarom ik in de radiotherapie ben beland. Het is fantastisch om bij een instituut te werken waar je regelmatig spontaan van patiënten te horen krijgt dat ze zo ontzettend tevreden zijn over alle medewerkers. Een speciaal woord van dank voor mijn directe collega's van cluster 1 . Zonder jullie bereidwilligheid regelmatig mijn klinische taken over te nemen, was onderzoek doen niet mogelijk geweest. De typische abdomen 
humor, die erger wordt naarmate de week vordert, geeft een goed tegenwicht tegen de ernstige kanten van ons werk.

Data Centre Maastro en trial poli, zonder jullie is klinisch onderzoek onmogelijk. Dank voor al het geduld dat jullie hebben met de toch niet altijd even nauwgezette artsen als het gaat om onderzoeksformaliteiten. In het bijzonder wil ik Ann danken: de laatste jaren ben jij zo ongeveer uitgegroeid tot de "privé datamanager" van het GE-onderzoek. Dank voor de fijne samenwerking!

Nancy en Jos van klinische chemie, enorm bedankt voor de snelle en deskundige bepaling van alle bloed biomarkers.

Beste Ankie, heel erg bedankt voor het mooie omslagontwerp! Heel bijzonder dat jij er voor hebt gezorgd! Beste Marlou, reuze bedankt voor het verzorgen van de fraaie lay out!

Beste Angela, collega, kamergenoot, vraagbaak, vertrouwenspersoon, vriendin. Samen begonnen bij Maastro, op dezelfde dag gesolliciteerd, samen opgeleid. Heel veel dank voor je adviezen en luisterend oor en natuurlijk de (culinaire) gezelligheid buiten het werk. Fijn dat je naast me wilt staan tijdens de verdediging!

Beste Frans, als je me in 1992 gevraagd had wie van ons de meeste kans zou maken ooit te promoveren, had ik op jou gegokt. Nooit zal ik vergeten hoe het voelde als de avond voor een proefwerk de telefoon ging (bij gebrek aan social media in die tijd). Ik dacht de stof volkomen te doorgronden en jij kwam vervolgens met een serie vragen, waar ik nog in de verste verte niet aan gedacht had. Jij hebt echter je liefde voor alles wat met radio en tv te maken heeft gevolgd en het promoveren aan je vrouw over gelaten. Dank dat je mijn paranimf wilt zijn! 
Mijn ouders wil ik bedanken voor de mogelijkheden die zij mij hebben geboden. Beste pa, wat zou je ontzettend trots zijn geweest als je dit boekje had kunnen zien. Ik kan nu niet meer zeggen "dat hebben we nog niet gehad", maar zou nu zeggen "dat weten we nog niet". Beste ma, ik heb bewondering voor hoe jij je leven weer hebt opgepakt en opnieuw hebt ingericht. Beste Piet, het is fijn dat jij in onze familie bent gekomen en je vervult je rol als "stief"vader/-opa geweldig! De JDI-club heeft zijn taak vervuld, de leden hebben langzaam hun eigen leven opgebouwd. Door de afstand zien we elkaar niet wekelijks, maar het is telkens weer een gezellig weerzien op Brabantse of Limburgse grond. Daan en Ivo, we blijven een bijzonder clubje.

Mijn schoonouders wil ik bedanken voor de wijze waarmee ze mij hebben opgenomen in de familie, het voelt helemaal thuis bij jullie. Beste Paul, ik vind het heel bijzonder dat jij als schoonvader een van de studies in dit proefschrift hebt mogelijk gemaakt en daarmee co-auteur bent. Chère Marie-Françoise, un grand merci pour tout ce que tu fais pour nous et bien sûr pour la bonne cuisine!

Lieve Cécile, zonder jou was dit proefschrift er nooit gekomen. Eigenlijk verdien jij een ere-doctoraat voor de manier waarop je al je activiteiten combineert en ons hele gezinsleven organiseert. Ik zeg te weinig hoe enorm ik dat waardeer. Lieve Matthijs, Anne, Wouter en Sophie, ons prachtige viertal. Ik ben ontzettend trots op jullie en de manier waarop jullie open staan voor het ontdekken van nieuwe dingen. Ik hou van jullie! 
Appendix

Curriculum vitae 
Jeroen Buijsen werd geboren op 30 juni 1974 te Halsteren en hij groeide op in Heerle. In 1992 behaalde hij het gymnasiumdiploma aan het Norbertuscollege te Roosendaal. Aansluitend startte hij met de opleiding geneeskunde aan de Universiteit Maastricht, waar hij in 1996 het doctoraaldiploma haalde (cum laude) en in 1998 zijn artsexamen deed (met genoegen). Zijn ervaringen tijdens de co-schappen legde hij vast in een serie columns voor het Algemeen Dagblad. $\mathrm{Na}$ zijn artsexamen werkte hij ruim 2 jaar als arts-onderzoeker bij de afdeling interne geneeskunde/endocrinologie van het azM, onder begeleiding van $\mathrm{dr}$. B. Wolffenbuttel. In die tijd bleef hij een medische rubriek schrijven voor het Algemeen Dagblad. In 2001 besloot hij de overstap te maken naar de radiotherapie en begon hij aan de opleiding tot radiotherapeut-oncoloog bij het RTIL in Heerlen (het huidige Maastro) met als opleiders dr. J. Jager en prof. dr. P. Lambin. De opleiding werd voltooid in 2006, waarna hij werd aangesteld als staflid bij Maastro. In die tijd zijn ook de eerste onderzoeksactiviteiten gestart die hebben geleid tot dit proefschrift. Het onderzoek werd begeleid door prof.dr. P. Lambin, dr. G. Lammering en dr. M. Öllers. Zijn huidige aandachtsgebieden zijn gastro-intestinale tumoren, mammatumoren en radiotherapie voor benigne aandoeningen. 
Appendix

List of publications 


\section{This thesis}

Development and external validation of a predictive model for pathological complete response of rectal cancer patients including sequential PET-CT imaging | Van Stiphout RG, Lammering G, Buijsen J, Janssen MH, Gambacorta MA, Slagmolen P, Lambrecht M, Rubello D, Gava M, Giordano A, Postma EO, Haustermans K, Capirci C, Valentini V, Lambin P | Radiother Oncol. 2011 Jan; 98(1):126-33.

FDG-PET provides the best correlation with the tumor specimen compared to MRI and CT in rectal cancer | Buijsen J, van den Bogaard J, Janssen MH, Bakers FC, Engelsman S, Öllers M, Beets-Tan RG, Nap M, Beets GL, Lambin P, Lammering G | Radiother Oncol. 2011 Feb;98(2):270-6.

FDG-PET-CT reduces the interobserver variability in rectal tumor delineation | Buijsen J, van den Bogaard J, van der Weide H, Engelsman S, van Stiphout R, Janssen M, Beets G, Beets-Tan R, Lambin P, Lammering G | Radiother Oncol. 2012 Mar;102(3):371-6.

Phase I trial of the combination of the Akt inhibitor nelfinavir and chemoradiation for locally advanced rectal cancer | Buijsen J, Lammering G, Jansen RL, Beets GL, Wals J, Sosef M, Den Boer MO, Leijtens J, Riedl RG, Theys J, Lambin P | Radiother Oncol. 2013 May;107(2):184-8.

Blood biomarkers are helpful in the prediction of response to chemoradiation in rectal cancer: a prospective, hypothesis driven study on patients with locally advanced rectal cancer | Buijsen J, van Stiphout RG, Menheere PP, Lammering G, Lambin P | Radiother Oncol. 2014 May;111(2):237-42.

A phase I-II study on the combination of rapamycin and short course radiotherapy in rectal cancer | Buijsen J, Van den Bogaard J, Jutten B, Belgers E, Sosef M.N., Leijtens JWA, Beets GL, Jansen RL, Riedl RG, Clarijs RG, Lammering GL, Lambin P | Submitted 


\section{Other}

Can perfusion MRI predict response to preoperative treatment in rectal cancer? | Martens MH, Subhani S, Heijnen LA, Lambregts DM, Buijsen J, Maas M, Riedl RG, Jeukens CR, Beets GL, Kluza E, Beets-Tan RG | Radiother Oncol. 2014 [Epub ahead of print]

Image-guided stereotactic ablative radiotherapy for the liver: A safe and effective treatment | Van De Voorde L, Vanneste B, Houben R, Damen P, van den Bogaard J, Lammering G, Dejong K, de Vos-Geelen J, Buijsen J, Ollers M, Berbée M, Lambin P | Eur J Surg Oncol. 2014 [Epub ahead of print]

Nomogram predicting response after chemoradiotherapy in rectal cancer using sequential PETCT imaging: A multicentric prospective study with external validation | van Stiphout RG, Valentini V, Buijsen J, Lammering G, Meldolesi E, van Soest J, Leccisotti L, Giordano A, Gambacorta MA, Dekker A, Lambin P | Radiother Oncol. 2014 Nov;113(2):215-22.

A qualitative synthesis of the evidence behind elective lymph node irradiation in oesophageal cancer | Van De Voorde L, Larue RT, Pijls M, Buijsen J, Troost EG, Berbée M, Sosef M, van Elmpt W, Schraepen MC, Vanneste B, Oellers M, Lambin P | Radiother Oncol. 2014 Nov;113(2):166-174. Review

MRI and Diffusion-Weighted MRI Volumetry for Identification of Complete Tumor Responders After Preoperative Chemoradiotherapy in Patients With Rectal Cancer: A Bi-institutional Validation Study | Lambregts DM, Rao SX, Sassen S, Martens MH, Heijnen LA, Buijsen J, Sosef M, Beets GL, Vliegen RA, Beets-Tan RG | Ann Surg. 2014 Sep 10. [Epub ahead of print]

'Rapid Learning health care in oncology' - an approach towards decision support systems enabling customised radiotherapy' | Lambin P, Roelofs E, Reymen B, Velazquez ER, Buijsen J, Zegers CM, Carvalho S, Leijenaar RT, Nalbantov G, 
Oberije C, Scott Marshall M, Hoebers F, Troost EG, van Stiphout RG, van Elmpt W, van der Weijden T, Boersma L, Valentini V, Dekker A | Radiother Oncol. 2013 Oct;109(1):159-64

Active Breathing Control in Combination With Ultrasound Imaging: A Feasibility Study of Image Guidance in Stereotactic Body Radiation Therapy of Liver Lesions | Bloemen-van Gurp E, van der Meer S, Hendry J, Buijsen J, Visser P, Fontanarosa D, Lachaine M, Lammering G, Verhaegen F | Int J Radiat Oncol Biol Phys. 2013 Mar 15;85(4):1096-102

Wait-and-See Policy for Clinical Complete Responders After Chemoradiation for Rectal Cancer | Maas M, Beets-Tan RG, Lambregts DM, Lammering G, Nelemans PJ, Engelen SM, van Dam RM, Jansen RL, Sosef M, Leijtens JW, Hulsewé KW, Buijsen J, Beets GL | J Clin Oncol. 2011 Dec 10;29(35):4633-40

Residual metabolic tumor activity after chemo-radiotherapy is mainly located in initially high FDG uptake areas in rectal cancer | Van den Bogaard J, Janssen MH, Janssens G, Buijsen J, Reniers B, Lambin P, Lammering G, Ollers MC | Radiother Oncol. 2011 May;99(2):137-41

Repeated positron emission tomography-computed tomography and perfusioncomputed tomography imaging in rectal cancer: fluorodeoxyglucose uptake corresponds with tumor perfusion | Janssen MH, Aerts HJ, Buijsen J, Lambin P, Lammering G, Ollers MC | Int J Radiat Oncol Biol Phys. 2012 Feb 1;82(2):849-55

PET-Based Treatment Response Evaluation in Rectal Cancer: Prediction and Validation | Janssen MH, Ollers MC, van Stiphout RG, Riedl RG, van den Bogaard J, Buijsen J, Lambin P, Lammering G | Int J Radiat Oncol Biol Phys. 2012 Feb 1;82(2):871-6

A systematic methodology review of phase I radiation dose escalation trials | Pijls-Johannesma M, van Mastrigt G, Hahn SM, De Ruysscher D, 
Baumert BG, Lammering G, Buijsen J, Bentzen SM, Lievens Y, Kramar A, Lambin P | Radiother Oncol. 2010 May;95(2): 135-41

Blood glucose level normalization and accurate timing improves the accuracy of PET-basedtreatment response predictions in rectal cancer | Janssen $\mathrm{MH}$, Ollers MC, van Stiphout RG, Riedl RG, van den Bogaard J, Buijsen J, Lambin P, Lammering G | Radiother Oncol. 2010May;95(2):203-8

Evaluation of early metabolic responses in rectal cancer during combined radiochemotherapyor radiotherapy alone: sequential FDG-PET-CT findings | Janssen MH, Ollers MC, vanStiphout RG, Buijsen J, van den Bogaard J, de Ruysscher D, Lambin P, Lammering G | Radiother Oncol. 2010 Feb;94(2): 151-5

Combined use of hyperthermia and radiation therapy for treating locally advanced cervicalcarcinoma | Lutgens L, van der Zee J, Pijls-Johannesma M, De Haas-Kock DF, Buijsen J,Mastrigt GA, Lammering G, De Ruysscher DK, Lambin P | Cochrane Database Syst Rev.2010 Jan 20;(1):CD006377

Comparison between perfusion computed tomography and dynamic contrastenhancedmagnetic resonance imaging in rectal cancer | Kierkels RG, Backes WH, Janssen MH, BuijsenJ, Beets-Tan RG, Lambin P, Lammering G, Oellers MC, Aerts HJ | Int J Radiat Oncol BiolPhys. 2010 Jun 1 ;77(2):400-8

Accurate prediction of pathological rectal tumorresponse after 2 weeks of preoperative radiochemotherapy using FDG-PET-CT imaging | Janssen MHM, Ollers MC, Riedl RG, Van den Bogaard J, Buijsen J, Van Stiphout RGPM, Aerts HJWL, Lambin P, Lammering G | Int J Radiat Oncol Biol Phys 2010 Jun 1 ;77(2):392-9

Concomitant hyperthermia and radiationtherapy for treating locally advanced rectal cancer | De Haas-Kock DF, Buijsen J, Pijls-Johannesma M, Lutgens L, Lammering G, Van Mastrigt GA, De Ruysscher DK, Lambin P, Van der Zee J | Cochrane Database Syst Rev. 2009 Jul8;(3):CD006269 
Tumor Delineation Based on Time-Activity Curve DifferencesAssessed with Dynamic Fluorodeoxyglucose Positron Emission Tomography-ComputedTomography in Rectal Cancer Patients | Janssen MH, Aerts HJ, Ollers MC, Bosmans G, Lee JA, Buijsen J, De Ruysscher D, LambinP, Lammering G, Dekker AL | Int J Radiat Oncol Biol Phys 2009 Feb 1;73(2):456-65

Can an FDG-PET/CTPredict Tumor Clearance of the Mesorectal Fascia after Preoperative Chemoradiation ofLocally Advanced Rectal Cancer? | Vliegen RF, Beets-Tan RG, Vanhauten B, Driessen A, Oellers M, Kessels AG, Arens A, Beets GL, Buijsen J, van Baardwijk A, de Ruysscher D, Lammering G | Strahlenther Onkol 2008 Sep; 184(9):457-464

Tumor delineation based on time-activity curve differences assessed with dynamicfluorodeoxyglucose positron emission tomography-computed tomography in rectal cancerpatients | Janssen $\mathrm{MH}$, Aerts $\mathrm{HJ}$, Oilers MC, Bosmans G, Lee JA, Buijsen J, De Ruysscher D,Lambin P, Lammering G, Dekker AL | Int J Radiat Oncol Bioi Phys. 2009 Feb 1 ;73(2):456-65

Tumor delineation and cumulative dosecomputation in radiotherapy based on deformable registration of respiratory correlated CTimages of lung cancer patients | Orban de Xivry J, Janssens G, Bosmans G, De Craene M, Dekker A, Buijsen J, vanBaardwijk A, De Ruysscher D, Macq B, Lambin P | Radiother Oncol 2007 Nov;85(2):232-8

PET-CT scan based auto-contouring in non-small cell lung cancercorrelates with pathology and reduces interobserver variability in the delineation of theprimary tumor and involved nodal volumes | Van Baardwijk A, Bosmans G, Boersma L, Buijsen J, Wanders S, Hochstenbag M, VanSuylen R, Dekker A, Dehing-Oberije C, Houben R, Bentzen S, Van Kroonenburgh M, LambinP, De Ruysscher D | Int J Radiat Oncol Biol Phys 2007 Jul 1 ;68(3):771-8 
Prone breast irradiation for pendulous breasts: a comparative study | Buijsen J, Jager JJ, Bovendeerd J, Voncken R, Borger JH, Boersma LJ, Murrer LHP, Lambin P | Radiother Oncol.2007;82:337-340

An "in silico" clinical trial comparing free breathing, slow and respiratorycorrelated computed tomography in lung cancer patients | Bosmans G, Buijsen J, Dekker ALAJ, Velders M, Boersma LJ, De Ruysscher D, MinkenAWH, Lambin P | Radiother Oncol 2006;81(1):73-80

Outcomes andpatterns of failure in solitary plasmacytoma: a multicenter Rare Cancer Network study of 258 patients | Ozsahin M, Tsang RW, Poortmans P, Belkacemi Y, Bolla M, Dinçbas FO, Landmann C,Castelain B, Buijsen J, Curschmann J, Kadish SP, Kowalczyk A, Anacak Y, Hammer J,Nguyen TD, Studer G, Cooper R, Sengoz M, Scandolaro L, Zouhair A | Int J Radiat Oncol Biol Phys 2006 Jan 1;64(1):210-7

Modulation of cell deathin the tumor microenvironment | Wouters BG, Koritzinsky M, Chiu RK, Theys J, Buijsen J, Lambin P | Semin Radiat Oncol 2003:13:31-41 
